\title{
List of symbols
}

$a$ soil exponent (unitless). See table S25. 50

$a_{i}$ soil exponent per layer $i$ (unitless). See table S25. 50

$a_{u z}$ exponent for the unsaturated zone (unitless). See table S26. 51

$5 A$ water abstraction $\left(\mathrm{L}^{3} \mathrm{~L}^{-2} \mathrm{~T}^{-1}\right)$. See tables $\mathrm{S} 32, \mathrm{~S} 35.57,60$

$A_{\text {act }}^{g w}$ actual groundwater abstraction $\left(\mathrm{L}^{3} \mathrm{~L}^{-2} \mathrm{~T}^{-1}\right)$. See table S53. 78

$A_{\text {act }}^{\text {sw }}$ actual surface water abstraction $\left(\mathrm{L}^{3} \mathrm{~L}^{-2} \mathrm{~T}^{-1}\right)$. See table S53. 78

$A_{a c t, i r r}$ actual water abstraction for irrigation $\left(\mathrm{L}^{3} \mathrm{~L}^{-2} \mathrm{~T}^{-1}\right)$. See table S43. 68

$A_{d e m, d a m}$ mean annual total water demand of the dam $\left(\mathrm{L}^{3} \mathrm{~T}^{-1}\right)$. See table $\mathrm{S} 37.62$

$10 A_{\text {dem,5dcells }}$ long term averaged water demand of 5 donwstream cells of a reservoir $\left(\mathrm{L}^{3} \mathrm{~T}^{-1}\right)$. See table S37. 62

$A_{d e m, 5 d c e l l s, \text { month }}$ mean water demand of 5 downstream cells of a reservoir averaged over a specific month $\left(\mathrm{L}^{3} \mathrm{~T}^{-1}\right)$. See table S37. 62

$A_{d o m}$ water abstraction for domestic sector $\left(\mathrm{L}^{3} \mathrm{~L}^{-2} \mathrm{~T}^{-1}\right)$. See tables S35, S59, S60, S61, S62, S63, S64. 60, 84-89

$A_{d o m}^{a q}$ water abstraction from river for domestic sector, at the origin of an aqueduct $\left(\mathrm{L}^{3} \mathrm{~L}^{-2} \mathrm{~T}^{-1}\right)$. See table $\mathrm{S} 62.87$

$A_{d o m}^{g w}$ water abstraction from groundwater storage for domestic sector $\left(\mathrm{L}^{3} \mathrm{~L}^{-2} \mathrm{~T}^{-1}\right)$. See tables S29, S59, S60 S61, S77. 54, $84-86,102$

$A_{d o m}^{g w, n r w}$ water abstraction from non-renewable groundwater storage for domestic sector $\left(\mathrm{L}^{3} \mathrm{~L}^{-2} \mathrm{~T}^{-1}\right)$. See table S59. 84

$A_{d o m}^{g w, r w}$ water abstraction from renewable groundwater storage for domestic sector $\left(\mathrm{L}^{3} \mathrm{~L}^{-2} \mathrm{~T}^{-1}\right)$. See table S59. 84

$A_{d o m}^{\text {pond }}$ water abstraction from pond, from local reservoir, for domestic sector $\left(\mathrm{L}^{3} \mathrm{~L}^{-2} \mathrm{~T}^{-1}\right)$. See tables S35, S62. 60, 87

$20 A_{d o m}^{r e}$ water abstraction from reservoir for domestic sector $\left(\mathrm{L}^{3} \mathrm{~L}^{-2} \mathrm{~T}^{-1}\right)$. See tables S62, S79. 87, 104

$A_{d o m}^{r i}$ water abstraction from river for domestic sector $\left(\mathrm{L}^{3} \mathrm{~L}^{-2} \mathrm{~T}^{-1}\right)$. See tables $\mathrm{S} 43, \mathrm{~S} 62.71,87$

$A_{d o m}^{s w}$ water abstraction from surface water bodies for domestic sector $\left(\mathrm{L}^{3} \mathrm{~L}^{-2} \mathrm{~T}^{-1}\right)$. See tables $\mathrm{S} 43$, S59, S62, S63, S64, S78, S79, S80. 68, 84, 87-89, 103-105

$A_{d o m}^{u s w}$ water abstraction from unlimited (unspecified) surface water source for domestic sector $\left(\mathrm{L}^{3} \mathrm{~L}^{-2} \mathrm{~T}^{-1}\right)$. See table $\mathrm{S} 62$. 87

$A_{\text {dom,cons }}$ domestic consumption $\left(\mathrm{L}^{3} \mathrm{~L}^{-2} \mathrm{~T}^{-1}\right)$. See tables S60, S61, S63. 85, 86, 88

$A_{\text {dom,cons }}^{s w}$ domestic surface water consumption $\left(\mathrm{L}^{3} \mathrm{~L}^{-2} \mathrm{~T}^{-1}\right)$. See table S63. 88

$A_{d o m, c o n s}^{g w}$ domestic groundwater consumption $\left(\mathrm{L}^{3} \mathrm{~L}^{-2} \mathrm{~T}^{-1}\right)$. See table $\mathrm{S} 60.85$

$A_{d o m, d e m}$ water demand for domestic sector $\left(\mathrm{L}^{3} \mathrm{~T}^{-1}\right)$. See tables S59, S60, S61, S62, S63, S78, S79. 84-88, 103,104

$30 A_{d o m, l o s s}^{s w}$ domestic surface water losses $\left(\mathrm{L}^{3} \mathrm{~L}^{-2} \mathrm{~T}^{-1}\right)$. See table S63. 88

$A_{\text {dom,loss }}^{g w}$ domestic groundwater losses $\left(\mathrm{L}^{3} \mathrm{~L}^{-2} \mathrm{~T}^{-1}\right)$. See table $\mathrm{S} 60.85$ 
$A_{d o m, m o n t h}$ monthly water abstraction for domestic sector $\left(\mathrm{L}^{3} \mathrm{~L}^{-2} \mathrm{~T}^{-1}\right)$. See tables S37, S79. 62, 104 $A_{d o m, r f}$ return flow from domestic sector $\left(\mathrm{L}^{3} \mathrm{~L}^{-2} \mathrm{~T}^{-1}\right)$. See tables S60, S61, S63, S64. 85, 86, 88, 89 $A_{d o m, r f}^{g w}$ return flow from domestic groundwater abstraction $\left(\mathrm{L}^{3} \mathrm{~L}^{-2} \mathrm{~T}^{-1}\right)$. See tables $\mathrm{S} 61, \mathrm{~S} 60.85,86$ $A_{d o m, r f}^{s w}$ return flow from domestic surface water abstraction $\left(\mathrm{L}^{3} \mathrm{~L}^{-2} \mathrm{~T}^{-1}\right)$. See tables S64, S63. 88, 89 $A_{d o m}^{r e, g l o b a l}$ water abstraction from a global reservoir for domestic sector $\left(\mathrm{L}^{3} \mathrm{~L}^{-2} \mathrm{~T}^{-1}\right)$. See tables S35, S62. 60, 87 $A_{\text {ele }}^{s w}$ water abstraction from surface water bodies for electricity sector $\left(\mathrm{L}^{3} \mathrm{~L}^{-2} \mathrm{~T}^{-1}\right)$. See tables $\mathrm{S} 75, \mathrm{~S} 76, \mathrm{~S} 78, \mathrm{~S} 79, \mathrm{~S} 80.100$, 101, 103-105

$A_{\text {fgw }}$ fossil groundwater abstraction $\left(\mathrm{L}^{3} \mathrm{~L}^{-2} \mathrm{~T}^{-1}\right)$. See table S53. 78

$40 A_{g w}$ water abstraction from groundwater storage $\left(\mathrm{L}^{3} \mathrm{~L}^{-2} \mathrm{~T}^{-1}\right)$. See tables $\mathrm{S} 29, \mathrm{~S} 77.54,102$ $A_{g w, c o n s}$ groundwater consumption $\left(\mathrm{L}^{3} \mathrm{~L}^{-2} \mathrm{~T}^{-1}\right)$. See table S54. 79 $A_{g w, r w}$ water abstraction from renewable groundwater storage $\left(\mathrm{L}^{3} \mathrm{~L}^{-2} \mathrm{~T}^{-1}\right)$. See table S53. 78 $A_{h i l, G}$ household, industry, and livestock demand, at the grid cell $(\mathrm{G})\left(\mathrm{L}^{3} \mathrm{~L}^{-2} \mathrm{~T}^{-1}\right)$. See tables S78, S79. 103, 104 $A_{\text {ind }}$ water abstraction for industry sector $\left(\mathrm{L}^{3} \mathrm{~L}^{-2} \mathrm{~T}^{-1}\right)$. See tables S35, S81. 60, 106 $A_{i n d}^{g w}$ groundwater abstraction for industry sector $\left(\mathrm{L}^{3} \mathrm{~L}^{-2} \mathrm{~T}^{-1}\right)$. See tables S77, S69, S70. 94, 95, 102 $A_{\text {ind }}^{\text {pond }}$ water abstraction from pond, local reservoir, for industry sector $\left(\mathrm{L}^{3} \mathrm{~L}^{-2} \mathrm{~T}^{-1}\right)$. See tables $\mathrm{S} 35, \mathrm{~S} 72.60,97$ $A_{\text {ind }}^{r e}$ water abstraction from reservoir for industry sector $\left(\mathrm{L}^{3} \mathrm{~L}^{-2} \mathrm{~T}^{-1}\right)$. See table S79. 104 $A_{i n d}^{r i}$ water abstraction from river for industry sector $\left(\mathrm{L}^{3} \mathrm{~L}^{-2} \mathrm{~T}^{-1}\right)$. See tables $\mathrm{S} 46, \mathrm{~S} 72.71,97$ $A_{i n d}^{s w}$ surface water abstraction for industry sector $\left(\mathrm{L}^{3} \mathrm{~L}^{-2} \mathrm{~T}^{-1}\right)$. See tables S69, S72, S73, S75. 94, 97, 98 $A_{i n d, c o n s}^{s w}$ surface water consumption for industry sector $\left(\mathrm{L}^{3} \mathrm{~L}^{-2} \mathrm{~T}^{-1}\right)$. See tables $\mathrm{S} 73.98$ $A_{i n d, c o n s}^{g w}$ groundwater consumption for industry sector $\left(\mathrm{L}^{3} \mathrm{~L}^{-2} \mathrm{~T}^{-1}\right)$. See tables $\mathrm{S} 70.95$ $A_{\text {ind,dem }}$ water demand abstraction for industry sector $\left(\mathrm{L}^{3} \mathrm{~T}^{-1}\right)$. See tables S69, S78, S79. 94, 103, 104 $A_{\text {ind,month }}$ monthly water abstraction for industry sector $\left(\mathrm{L}^{3} \mathrm{~L}^{-2} \mathrm{~T}^{-1}\right)$. See tables $\mathrm{S} 37, \mathrm{~S} 79.62,104$ $A_{\text {ind }}^{\text {re,global }}$ water abstraction from a global reservoir for industry sector $\left(\mathrm{L}^{3} \mathrm{~L}^{-2} \mathrm{~T}^{-1}\right)$. See tables S35, S72. 60, 97

$A_{\text {irr }}$ water abstraction for irrigation sector $\left(\mathrm{L}^{3} \mathrm{~L}^{-2} \mathrm{~T}^{-1}\right)$. See tables S1, S35, S43, S52, S53, S54, S55, S56, S57, S58. 26, 60, 77, 79-83, 105

$A_{i r r}^{a q}$ water abstraction from river for irrigation sector, at the origin of an aqueduct $\left(\mathrm{L}^{3} \mathrm{~L}^{-2} \mathrm{~T}^{-1}\right)$. See table S56. 81

$A_{i r r}^{g w}$ water abstraction from groundwater storage for irrigation sector $\left(\mathrm{L}^{3} \mathrm{~L}^{-2} \mathrm{~T}^{-1}\right)$. See tables $\mathrm{S} 29, \mathrm{~S} 53, \mathrm{~S} 54, \mathrm{~S} 55, \mathrm{~S} 77.54$, 78-80, 102

$60 A_{i r r}^{g w, n r w}$ water abstraction from non-renewable groundwater storage for irrigation sector $\left(\mathrm{L}^{3} \mathrm{~L}^{-2} \mathrm{~T}^{-1}\right)$. See table S53. 78 $A_{i r r}^{g w, r w}$ water abstraction for irrigation sector taken from renewable groundwater storage $\left(\mathrm{L}^{3} \mathrm{~L}^{-2} \mathrm{~T}^{-1}\right)$. See table $\mathrm{S} 53.78$ 
$A_{i r r}^{l a}$ water abstraction for irrigation sector taken from lake $\left(\mathrm{L}^{3} \mathrm{~L}^{-2} \mathrm{~T}^{-1}\right)$. See table S56. 81

$A_{i r r}^{\text {neigh,cell }}$ water abstraction for irrigation sector taken from neighboring cell surplus $\left(\mathrm{L}^{3} \mathrm{~L}^{-2} \mathrm{~T}^{-1}\right)$. See table S56. 81

$A_{i r r}^{\text {pond }}$ water abstraction for irrigation sector taken from pond, local reservoir $\left(\mathrm{L}^{3} \mathrm{~L}^{-2} \mathrm{~T}^{-1}\right)$. See tables S35, S56. 60, 81

$65 A_{i r r}^{r e}$ water abstraction from reservoir for irrigation sector $\left(\mathrm{L}^{3} \mathrm{~L}^{-2} \mathrm{~T}^{-1}\right)$. See tables S56, S79. 81, 104

$A_{i r r}^{r i}$ water abstraction for irrigation from river $\left(\mathrm{L}^{3} \mathrm{~L}^{-2} \mathrm{~T}^{-1}\right)$. See tables $\mathrm{S} 46, \mathrm{~S} 56.71,81$

$A_{i r r}^{s w}$ water abstraction for irrigation sector taken from surface water $\left(\mathrm{L}^{3} \mathrm{~L}^{-2} \mathrm{~T}^{-1}\right)$. See tables S43, S53, S56, S57, S58, S78, S79, S80. 68, 78, 81-83, 103-105

$A_{i r r}^{u s w}$ irrigation surface water abstraction from unlimited (unspecified) surface water source $\left(\mathrm{L}^{3} \mathrm{~L}^{-2} \mathrm{~T}^{-1}\right)$. See table S56. 81

$70 A_{\text {irr,app }}$ application requirement for an irrigation system due to irrigation conveyance inefficiencies $\left(\mathrm{L}^{3} \mathrm{~L}^{-2} \mathrm{~T}^{-1}\right)$. See tables S57, S58. 82, 83

$A_{\text {irr,cons }}$ consumptive water use for irrigation sector $\left(\mathrm{L}^{3} \mathrm{~L}^{-2} \mathrm{~T}^{-1}\right)$. See tables S53, S54, S56, S57. 78, 79, 81, 82

$A_{i r r, c o n s}^{s w}$ irrigation surface water consumption $\left(\mathrm{L}^{3} \mathrm{~L}^{-2} \mathrm{~T}^{-1}\right)$. See tables S57, S58. 82, 83

$A_{i r r, c o n s}^{g w}$ irrigation groundwater consumption $\left(\mathrm{L}^{3} \mathrm{~L}^{-2} \mathrm{~T}^{-1}\right)$. See tables S54, S55. 79, 80

$75 A_{\text {irr,dem }}$ water demand for irrigation sector $\left(\mathrm{L}^{3} \mathrm{~T}^{-1}\right)$. See tables S53, S78, S79. 78, 103, 104

$A_{\text {irr,gross, } G}$ gross irrigation requirement, at the grid cell $(\mathrm{G})\left(\mathrm{L}^{3} \mathrm{~L}^{-2} \mathrm{~T}^{-1}\right)$. See tables S57, S58, S78, S79. 82, 83, 103,104

$A_{i r r, \text { loss }}^{\text {sw }}$ irrigation surface water losses $\left(\mathrm{L}^{3} \mathrm{~L}^{-2} \mathrm{~T}^{-1}\right)$. See table S57. 82

$A_{\text {irr, month }}$ monthly water abstraction for irrigation sector $\left(\mathrm{L}^{3} \mathrm{~L}^{-2} \mathrm{~T}^{-1}\right)$. See tables S37, S79. 62, 104

$A_{i r r, n e t}$ daily net irrigation requirement. Amount of water required in the upper $50 \mathrm{~cm}$ soil to avoid crop water limitation

$\left(\mathrm{L}^{3} \mathrm{~L}^{-2} \mathrm{~T}^{-1}\right)$. See tables S57, S58. 82, 83

$A_{i r r, r f}^{g w}$ return flow from irrigation groundwater abstraction $\left(\mathrm{L}^{3} \mathrm{~L}^{-2} \mathrm{~T}^{-1}\right)$. See table S55. 80

$A_{i r r, r f}^{s w}$ return flow from irrigation surface water abstraction $\left(\mathrm{L}^{3} \mathrm{~L}^{-2} \mathrm{~T}^{-1}\right)$. See tables S57, S58. 82, 83

$A_{i r r}^{r e, g l o b a l}$ water abstraction from a global reservoir for irrigation sector $\left(\mathrm{L}^{3} \mathrm{~L}^{-2} \mathrm{~T}^{-1}\right)$. See tables $\mathrm{S} 29, \mathrm{~S} 56.60,81$

$A_{l a}$ water abstraction from lake $\left(\mathrm{L}^{3} \mathrm{~L}^{-2} \mathrm{~T}^{-1}\right)$. See tables S43, S78, S79. 57, 103, 104

$A_{\text {liv }}^{g w}$ water abstraction for livestock sector taken from groundwater storage $\left(\mathrm{L}^{3} \mathrm{~L}^{-2} \mathrm{~T}^{-1}\right)$. See tables S29, S65. 54, 90

$A_{\text {liv }}^{s w}$ water abstraction for livestock sector taken from surface water $\left(\mathrm{L}^{3} \mathrm{~L}^{-2} \mathrm{~T}^{-1}\right)$. See tables $\mathrm{S} 43, \mathrm{~S} 67, \mathrm{~S} 78, \mathrm{~S} 79, \mathrm{~S} 80.68$, 92, 103-105

$A_{l i v, \text { cons }}$ water consumption for livestock sector $\left(\mathrm{L}^{3} \mathrm{~L}^{-2} \mathrm{~T}^{-1}\right)$. See tables S66, S68. 91,93

$A_{\text {liv,cons }}^{g w}$ water consumption use for livestock sector taken from groundwater $\left(\mathrm{L}^{3} \mathrm{~L}^{-2} \mathrm{~T}^{-1}\right)$. See table S66. 91

$A_{\text {liv,cons }}^{s w}$ water consumption use for livestock sector taken from surface water $\left(\mathrm{L}^{3} \mathrm{~L}^{-2} \mathrm{~T}^{-1}\right)$. See tables S67, S68. 92, 93

$A_{l i v, d e m}$ water demand for livestock sector $\left(\mathrm{L}^{3} \mathrm{~T}^{-1}\right)$. See tables S65, S66, S67, S68, S78, S79. 90-93, 103, 104

$A_{m a n, r f}^{g w}$ return flow for manufacturing groundwater abstraction $\left(\mathrm{L}^{3} \mathrm{~L}^{-2} \mathrm{~T}^{-1}\right)$. See tables $\mathrm{S} 71, \mathrm{~S} 70.95,96$ 
$A_{\text {man }, \text { rf }}^{\text {sw }}$ return flow for manufacturing from surface water abstraction $\left(\mathrm{L}^{3} \mathrm{~L}^{-2} \mathrm{~T}^{-1}\right)$. See tables $\mathrm{S} 74, \mathrm{~S} 73.98,99$

$A_{\text {man }}^{a q}$ water abstraction for manufacturing sector from river, at the origin of an aqueduct $\left(\mathrm{L}^{3} \mathrm{~L}^{-2} \mathrm{~T}^{-1}\right)$. See table S72. 97

$A_{m a n}^{c o o, c}$ manufacturing cooling water per country $\left(\mathrm{L}^{3} \mathrm{~T}^{-1}\right)$. See tables S70, S71, S73, S74. 95, 96, 98, 99

$A_{m a n}^{c}$ water abstraction for manufacturing sector per country $\left(\mathrm{L}^{3} \mathrm{~T}^{-1}\right)$. See tables S69, S70, S72, S73. 94, 95, 97,98

$A_{\text {man }}^{\text {gw,nrw }}$ water abstraction for manufacturing sector taken from non-renewable groundwater storage $\left(\mathrm{L}^{3} \mathrm{~L}^{-2} \mathrm{~T}^{-1}\right)$. See table S69. 94

100

$A_{\text {man }}^{g w, r w}$ water abstraction for manufacturing sector taken from renewable groundwater storage $\left(\mathrm{L}^{3} \mathrm{~L}^{-2} \mathrm{~T}^{-1}\right)$. See table S69. 94

$A_{m a n}^{r e}$ water abstraction from reservoir for manufacturing sector $\left(\mathrm{L}^{3} \mathrm{~L}^{-2} \mathrm{~T}^{-1}\right)$. See table $\mathrm{S} 72.97$

$A_{\text {man }}^{r i}$ water abstraction from river for manufacturing sector $\left(\mathrm{L}^{3} \mathrm{~L}^{-2} \mathrm{~T}^{-1}\right)$. See table S72. 97

$A_{\text {man }}^{\text {sw }}$ water abstraction for manufacturing sector taken from surface water $\left(\mathrm{L}^{3} \mathrm{~L}^{-2} \mathrm{~T}^{-1}\right)$. See tables S43, S72, S73, S74, S78, S79, S80. 68, 97-99, 103-105

$A_{m a n}^{u s w}$ manufacturing surface water abstraction from unlimited (unspecified) surface water source $\left(\mathrm{L}^{3} \mathrm{~L}^{-2} \mathrm{~T}^{-1}\right)$. See table S72. 97

$A_{\text {man }}^{w w, c}$ manufacturing wastewater per country $\left(\mathrm{L}^{3} \mathrm{~T}^{-1}\right)$. See tables S70, S71, S73, S74. 95, 96, 98, 99

$A_{\text {man,cons }}$ water consumption for manufacturing sector $\left(\mathrm{L}^{3} \mathrm{~L}^{-2} \mathrm{~T}^{-1}\right)$. See tables S70, S71, S73. 95, 96, 98

$A_{\text {man,cons }}^{g w}$ water consumption for manufacturing sector taken from groundwater storage $\left(\mathrm{L}^{3} \mathrm{~L}^{-2} \mathrm{~T}^{-1}\right)$. See table S70. 95

$A_{\text {man,cons }}^{\text {sw }}$ water consumption for manufacturing sector taken from surface water $\left(\mathrm{L}^{3} \mathrm{~L}^{-2} \mathrm{~T}^{-1}\right)$. See table S73. 98

$A_{\text {man,dem }}$ water demand for manufacturing sector $\left(\mathrm{L}^{3} \mathrm{~T}^{-1}\right)$. See tables S69, S70, S71, S72 S73. 94-98

$A_{\text {man }, \text { loss }}^{\text {gw }}$ manufacturing groundwater losses $\left(\mathrm{L}^{3} \mathrm{~L}^{-2} \mathrm{~T}^{-1}\right)$. See table S70. 95

$A_{\text {man,loss }}^{\text {sw }}$ manufacturing surface water losses $\left(\mathrm{L}^{3} \mathrm{~L}^{-2} \mathrm{~T}^{-1}\right)$. See table S73. 98

$A_{m a n, r f}^{c}$ return flow for manufacturing abstraction per country $\left(\mathrm{L}^{3} \mathrm{~L}^{-2} \mathrm{~T}^{-1}\right)$. See tables $\mathrm{S} 71, \mathrm{~S} 70.95,98$

$A_{\text {man }}^{g w}$ water abstraction for manufacturing sector taken from groundwater storage $\left(\mathrm{L}^{3} \mathrm{~L}^{-2} \mathrm{~T}^{-1}\right)$. See tables $\mathrm{S} 29, \mathrm{~S} 69, \mathrm{~S} 70$, S71. 54, 94-96

$A_{\text {muni }}$ water abstraction for municipal sector $\left(\mathrm{L}^{3} \mathrm{~L}^{-2} \mathrm{~T}^{-1}\right)$. See table S81. 106

$A_{\text {ocean }}$ seawater abstraction $\left(\mathrm{L}^{3} \mathrm{~L}^{-2} \mathrm{~T}^{-1}\right)$. See tables S81, S82, S83. 106-108

$A_{\text {ocean,cons }}$ seawater consumption $\left(\mathrm{L}^{3} \mathrm{~L}^{-2} \mathrm{~T}^{-1}\right)$. See table S82. 107

$A_{\text {ocean }, r f}$ return flow from seawater abstraction $\left(\mathrm{L}^{3} \mathrm{~L}^{-2} \mathrm{~T}^{-1}\right)$. See table S83. 108

$A_{\text {pot }}^{g w}$ potential groundwater abstraction $\left(\mathrm{L}^{3} \mathrm{~L}^{-2} \mathrm{~T}^{-1}\right)$. See table S53. 78

$A_{r e}$ water abstraction from local reservoir $\left(\mathrm{L}^{3} \mathrm{~L}^{-2} \mathrm{~T}^{-1}\right)$. See tables S35, S79. 60, 104

$A_{r f}$ return flow from water abstraction $\left(\mathrm{L}^{3} \mathrm{~L}^{-2} \mathrm{~T}^{-1}\right)$. See table S32. 57, 60, 68

$A_{r i}$ water abstraction from river $\left(\mathrm{L}^{3} \mathrm{~L}^{-2} \mathrm{~T}^{-1}\right)$. See tables $\mathrm{S} 43, \mathrm{~S} 80.68,105$ 
$A_{\text {sw,cons }}$ surface water consumption $\left(\mathrm{L}^{3} \mathrm{~L}^{-2} \mathrm{~T}^{-1}\right)$. See table S54. 79

$A_{\text {tot }}$ total abstraction $\left(\mathrm{L}^{3} \mathrm{~L}^{-2} \mathrm{~T}^{-1}\right)$. See tables S29, S53. 54, 78

$A_{\text {tot,cons }}$ total consumption $\left(\mathrm{L}^{3} \mathrm{~L}^{-2} \mathrm{~T}^{-1}\right)$. See table S54. 79

AET total amount of water from transpiration, evaporation, interception losses, and sublimation $\left(\mathrm{L}^{3} \mathrm{~L}^{-2} \mathrm{~T}^{-1}\right)$. See tables S1, S11, S20. 26, 36, 45

$b_{\text {weir }}$ width weir (L). See tables S34, S37. 59, 62

$B$ area $\left(\mathrm{L}^{2}\right)$. See tables $\mathrm{S} 35, \mathrm{~S} 46.60,71$

$B_{\text {calm }}$ fraction of the calm areas (unitless). See table S21. 46

$B_{G}$ area of a grid cell $\left(\mathrm{L}^{2}\right)$. See table S29, S43. 54, 68

$B_{l a, g l o b a l}$ global lake area $\left(\mathrm{L}^{2}\right)$. See table S32. 57

$B_{\text {la,global,max }}$ maximum global lake area $\left(\mathrm{L}^{2}\right)$. See tables S32, S49. 57, 76

$B_{\text {la,local }}$ local lake area $\left(\mathrm{L}^{2}\right)$. See table S32. 57

$B_{l a, l o c a l, \text { max }}$ maximum local lake area $\left(\mathrm{L}^{2}\right)$. See tables S32, S49. 57, 76

$B_{r e}$ reservoir area $\left(\mathrm{L}^{2}\right)$. See tables $\mathrm{S} 35, \mathrm{~S} 38.60,63$

$B_{r e, \max }$ maximum reservoir area $\left(\mathrm{L}^{2}\right)$. See table $\mathrm{S} 35.60$

$140 B_{\text {stormy }}$ fraction of the stormy areas (unitless). See tables S4, S21. 29, 46

$B_{w e, g l o b a l}$ global wetland area $\left(\mathrm{L}^{2}\right)$. See table S39. 64

$B_{\text {we,global,max }}$ maximum global wetland area $\left(\mathrm{L}^{2}\right)$. See tables S39, S49. 64, 76

$B_{\text {we,local }}$ local wetland area $\left(\mathrm{L}^{2}\right)$. See table S39. 64

$B_{\text {we,local,max }}$ maximum local wetland area $\left(\mathrm{L}^{2}\right)$. See tables S39, S49. 64, 76

$145 c_{\text {air }}$ specific heat of air (unitless). See tables S2, S7, S11, S23, S24. 27, 32, 36, 48, 49

$c_{i c e}$ specific heat ice (unitless). See tables S10, S12. 35, 37

$C_{\text {bulk }}$ bulk transfer coefficient (unitless). See table S2. 27

$C_{b u l k, E g}$ bulk coefficient for evaporation from snow-free ground (unitless). See table S11. 36

$C_{b u l k, E g}^{s n}$ bulk coefficient for evaporation from snow-covered ground (unitless). See table S11. 36

$C_{b u l k, E i}$ bulk coefficient for evaporation, from intercepted liquid water by canopy (unitless). See table S7. 32

$C_{b u l k, E i}^{s n}$ bulk coefficient for evaporation, from intercepted snow by canopy (unitless). See table S7. 32

$C_{B, \text { red }}$ area reduction factor (unitless). See tables S32, S35, S39. 57, 60, 64

$C_{\text {crop }}$ crop coefficient (unitless). See tables S23, S24. 48, 49

$C_{\text {cropGN }}$ crop group number (GN) is an indicator of adaptation to dry climate (unitless). See table S23. 48 
$C_{d a m, G}$ allocation coefficient for grid cell that can be supply by more than one dam (unitless). See table S37. 62

$C_{d o m}^{w u, i n t s}$ domestic water use intensity $\left(\mathrm{L}^{3}\right.$ capita $\left.^{-1} \mathrm{~T}^{-1}\right)$. See tables S59, S62. 84, 87

$C_{d o m, \text { cons }}$ domestic consumptive use coefficient (unitless). See tables S60, S61, S63, S64. 85, 86, 88, 89

$C_{\text {ele }}^{\text {techchangerate }}$ technological change rate for the electricity sector (unitless). See tables S75, S76. 100, 101

$C_{g w, Q}$ groundwater outflow coefficient or recession coefficient of groundwater zone $\left(\mathrm{T}^{-1}\right)$. See table $\mathrm{S} 31.56$

$C_{H}$ surface exchange coefficient for sensible and latent heat fluxes between the surface and the lowest atmospheric level (unitless). See tables S23, S24. 48, 49

$C_{i c e}$ ice impedance coefficient determined from the ice content of the soil layers (unitless). See table S31. 56

$C_{\text {liv }}^{w, r e q}$ livestock specific animal water requirement $\left(\mathrm{L}^{3}\right.$ capita $\left.{ }^{-1} \mathrm{~T}^{-1}\right)$. See table S68. 93

$C_{m}$ degree-day factor $\left(\mathrm{L} \Theta^{-1} \mathrm{~T}^{-1}\right)$. See table S12. 37

$C_{m, \text { season }}$ seasonal degree-day factor (unitless). See table S12. 37

$C_{m a n}^{w, i n t s, 2005}$ manufacturing structural water intensity of 2005 per country $\left(\mathrm{L}^{3}\right.$ money $\left.{ }^{-1}\right)$. See tables S69, S70, S72, S73. 94, 95, 97, 98

$C_{\text {man,cons }}$ manufacturing consumptive use coefficient (unitless). See tables S73, S70, S74, S71. 95, 96, 98, 99

$C_{m a n}^{t e c h, c r}$ technological change rate for the manufacturing sector (unitless). See tables S69, S70, S72, S73. 94, 95, 97, 98

$C_{\text {month }}$ monthly provisional release coefficient $\left(\mathrm{L}^{-3} \mathrm{~T}^{-1}\right)$. See table S37. 62

$C_{M}$ Manning's roughness coefficient for river bed (unitless). See table S46. 71

$C_{M 1}$ Manning coefficient 1 (unitless). See table S46. 71

$C_{M 2}$ Manning coefficient 2 (unitless). See table S46. 71

$C_{M 3}$ Manning coefficient 3 (unitless). See table S46. 71

$C_{M S, w e}$ Manning-Strickler coefficient for wetlands (unitless). See table S39. 67

$C_{P, l}$ extinction coefficient for rainfall (unitless). See table S5, S4. 29, 30

$C_{P_{t h}}$ throughfall coefficient (unitless). See table S5. 30

$C_{P T}$ Priestley-Taylor coefficient: 1.26 in humid areas, 1.74 in semiarid / arid areas Kaspar [26] (unitless). See table S2. 27

$C_{r i, h y d r a u l i c}$ hydraulic radius coefficient of the river channel (L). See table S46. 71

$C_{s o, F}$ reduction factor for frozen soil (unitless). See table S13. 38

$C_{s o, i}$ soil matric potential coefficient (unitless). See table S28. 53

$C_{s u}$ surface drag coefficient (unitless). See tables S7, S11, S23, S24. 32, 36, 48, 49

$C_{s w, \text { out }}$ surface water outflow coefficient $\left(\mathrm{T}^{-1}\right)$. See tables S34, S42. 59, 67 
$C_{\text {tot }}$ total annual release coefficient $\left(\mathrm{L}^{-3} \mathrm{~T}^{-1}\right)$. See table S37. 62

$C_{w e i r}$ coefficient friction of the reservoir weir (unitless). See tables S34, S37, S45, S46. 59, 62, 70, 71

$C_{w s}$ reduction factor because of water stress (unitless). See table S23. 48

$C_{\text {year }}$ yearly release coefficient $\left(\mathrm{L}^{-3} \mathrm{~T}^{-1}\right)$. See table $\mathrm{S} 37.62$

$d_{0}$ threshold depth (L). See table S31. 56

$d_{n, i}$ layer $i$ node depth (L). See table S13. 38

$d_{n, i+1}$ layer $i+1$ node depth (L). See table S13. 38

$d_{\text {paddy }}^{s w}$ actual surface water depth for paddy irrigation (L). See table S57. 82

$d_{\text {paddy,max }}^{s w}$ maximal surface water depth for paddy irrigation $(\mathrm{L})(50 \mathrm{~mm})$. See table S57. 82

$d_{s n}$ grid-average of depth of the snow cover (L). See table S13. 38

$d_{\text {so }}$ total soil depth (L). See tables S14, S20, S25, S29, S30. 39, 45, 50, 54, 55

$d_{s o, i}$ soil depth for layer index $i(\mathrm{~L})$. See tables S14, S24, S25, S49. 39, 49, 50, 75

$d_{\text {so,root }}$ rooting depth (L). See tables S18, S22, S49. 43, 47, 75

$d_{w t}$ water table depth (L). See tables S29, S30, S31. 54-56

$d_{w t, i}$ depth of the layer $i$ directly above the water table (L). See table S30. 55

$D_{w}$ soil water diffusivity $\left(\mathrm{L}^{2} \mathrm{~T}^{-1}\right)$. See table S14. 39

$e$ vapor pressure $(\mathrm{kPa})$. See tables $\mathrm{S} 7, \mathrm{~S} 11, \mathrm{~S} 23, \mathrm{~S} 24.32,36,48,49$

$e_{a c t}$ actual vapor pressure $(\mathrm{kPa})$. See table S2. 27

$e_{c a}$ vapor pressure in canopy air space (kPa). See tables S7, S11, S23, S24. 32, 36, 48, 49

$e_{\text {sat }}$ saturation vapor pressure (kPa); for Mac-PDM.20 (mb). See table S2. 27

$e_{\text {sat,dew }}$ saturation vapor pressure at dew point; for Mac-PDM.20 (mb). See table S2. 27

$E_{c a}$ evaporation, water changes from liquid to vapour, from canopy storage $\left(\mathrm{L}^{3} \mathrm{~L}^{-2} \mathrm{~T}^{-1}\right)$. See tables $\mathrm{S} 1, \mathrm{~S} 3, \mathrm{~S} 5, \mathrm{~S} 7, \mathrm{~S} 9, \mathrm{~S} 23$, S24. 26, 28, 30, 32, 34, 45, 49

$E_{c a}^{s n, c o v}$ evaporation from snow-covered canopy $\left(\mathrm{L}^{3} \mathrm{~L}^{-2} \mathrm{~T}^{-1}\right)$. See table S1. 26

$E_{c a}^{s n, f r e e}$ evaporation from snow-free canopy $\left(\mathrm{L}^{3} \mathrm{~L}^{-2} \mathrm{~T}^{-1}\right)$. See table $\mathrm{S} 1.26$

$210 E_{c a, l}$ amount of water, from rainfall, accumulated on the vegetation that changes from liquid to vapor $\left(\mathrm{L}^{3} \mathrm{~L}^{-2} \mathrm{~T}^{-1}\right)$. See tables S3, S7, S10. 28, 32, 35

$E_{c a, \max }$ maximum amount of water accumulated on the vegetation that changes from liquid to vapor $\left(\mathrm{L}^{3} \mathrm{~L}^{-2} \mathrm{~T}^{-1}\right)$. See table S7. 32

$E_{c a, s}$ amount of water, from snowfall, accumulated on the vegetation that changes from solid to vapor $\left(\mathrm{L}^{3} \mathrm{~L}^{-2} \mathrm{~T}^{-1}\right)$. See tables S3, S7, S9. 28, 32, 34 
$E_{\text {dew }}$ liquid dew, accumulated on the ground or snow that changes from vapor to liquid $\left(\mathrm{L}^{3} \mathrm{~L}^{-2} \mathrm{~T}^{-1}\right)$. See tables $\mathrm{S} 8, \mathrm{~S} 10.33$, 35

$E_{e q}$ evaporation at the equilibrium $\left(\mathrm{L}^{3} \mathrm{~L}^{-2} \mathrm{~T}^{-1}\right)$. See table $\mathrm{S} 24.49$

$E_{\text {floodplain }}$ evaporation from floodplain, it can be wetland $\left(\mathrm{L}^{3} \mathrm{~L}^{-2} \mathrm{~T}^{-1}\right)$. See table S1. 26

$E_{l a}$ evaporation from lake storage $\left(\mathrm{L}^{3} \mathrm{~L}^{-2} \mathrm{~T}^{-1}\right)$. See tables $\mathrm{S} 1, \mathrm{~S} 32, \mathrm{~S} 33, \mathrm{~S} 45, \mathrm{~S} 47.26,57,58,70,72$

$E_{l a, p o t}$ potential evaporation from lake storage $\left(\mathrm{L}^{3} \mathrm{~L}^{-2} \mathrm{~T}^{-1}\right)$. See tables S33, S47. 58, 72

$E_{l f s m}$ leaf and stem surface evaporation $\left(\mathrm{L}^{3} \mathrm{~L}^{-2} \mathrm{~T}^{-1}\right)$. See table S9. 34

$E_{\text {osw }}$ evaporation from open water surfaces $\left(\mathrm{L}^{3} \mathrm{~L}^{-2} \mathrm{~T}^{-1}\right)$. See tables S57, S58. 82, 83

$E_{r e}$ evaporation from reservoir storage $\left(\mathrm{L}^{3} \mathrm{~L}^{-2} \mathrm{~T}^{-1}\right)$. See tables S1, S35, S38, S45. 26, 60, 63, 70

$E_{r e, p o t}$ potential evaporation from reservoir storage $\left(\mathrm{L}^{3} \mathrm{~L}^{-2} \mathrm{~T}^{-1}\right)$. See table S38. 63

$E_{r i}$ evaporation from river storage $\left(\mathrm{L}^{3} \mathrm{~L}^{-2} \mathrm{~T}^{-1}\right)$. See tables $\mathrm{S} 1, \mathrm{~S} 47.26,72$

$E_{s n}$ sublimation, water that changes from solid (snow and ice) to vapor $\left(\mathrm{L}^{3} \mathrm{~L}^{-2} \mathrm{~T}^{-1}\right)$. See tables $\mathrm{S} 1, \mathrm{~S} 8, \mathrm{~S} 9, \mathrm{~S} 11, \mathrm{~S} 12.26$, $33,34,36,37$

$E_{s n}^{\text {covgr }}$ sublimation on snow covered ground $\left(\mathrm{L}^{3} \mathrm{~L}^{-2} \mathrm{~T}^{-1}\right)$. See table $\mathrm{S} 11.36$

$E_{s n}^{\text {freegr }}$ sublimation on snow free ground $\left(\mathrm{L}^{3} \mathrm{~L}^{-2} \mathrm{~T}^{-1}\right)$. See table $\mathrm{S} 11.36$

$E_{s n, s o}$ sublimation on soil, water that changes from solid (snow and ice) to vapor $\left(\mathrm{L}^{3} \mathrm{~L}^{-2} \mathrm{~T}^{-1}\right)$. See tables $\mathrm{S} 10.35$

$E_{s n, S G_{i}}$ sublimation in a subgrid cell Müller Schmied et al. [34] $\left(\mathrm{L}^{3} \mathrm{~L}^{-2} \mathrm{~T}^{-1}\right)$. See tables $\mathrm{S} 8, \mathrm{~S} 11.33,36$

$E_{\text {snunderca }}$ sublimation under canopy, water that changes from solid (snow and ice) to vapor $\left(\mathrm{L}^{3} \mathrm{~L}^{-2} \mathrm{~T}^{-1}\right)$. See table $\mathrm{S} 10$, S11. 35, 36

$E_{\text {so }}$ soil evaporation, water changes from liquid to vapor $\left(\mathrm{L}^{3} \mathrm{~L}^{-2} \mathrm{~T}^{-1}\right)$. See tables $\mathrm{S} 1, \mathrm{~S} 8, \mathrm{~S} 11, \mathrm{~S} 14, \mathrm{~S} 23, \mathrm{~S} 24, \mathrm{~S} 25.26,33$, $36,39,48-50$

$E_{s o, i}$ soil evaporation from soil layer $i\left(\mathrm{~L}^{3} \mathrm{~L}^{-2} \mathrm{~T}^{-1}\right)$. See table S14. 39

$E_{\text {so, ice }}$ soil evaporation from ground covered with ice $\left(\mathrm{L}^{3} \mathrm{~L}^{-2} \mathrm{~T}^{-1}\right)$. See table $\mathrm{S} 1.26$

$E_{\text {so,l }, \text { freegr }}^{\text {sn, }}$ soil evaporation from snow free ground $\left(\mathrm{L}^{3} \mathrm{~L}^{-2} \mathrm{~T}^{-1}\right)$. See tables S1, S24. 26, 49

$240 E_{w e}$ evaporation from wetland storage $\left(\mathrm{L}^{3} \mathrm{~L}^{-2} \mathrm{~T}^{-1}\right)$. See tables $\mathrm{S} 1, \mathrm{~S} 39, \mathrm{~S} 41.26,64,66$

$E T_{s o, i}$ evapotranspiration from soil layer $i\left(\mathrm{~L}^{3} \mathrm{~L}^{-2} \mathrm{~T}^{-1}\right)$. See tables $\mathrm{S} 1, \mathrm{~S} 14, \mathrm{~S} 24.26,39,49$

$E T_{s o, i-1}$ evapotranspiration from soil layer $i-1\left(\mathrm{~L}^{3} \mathrm{~L}^{-2} \mathrm{~T}^{-1}\right)$. See table $\mathrm{S} 24.49$

$f$ fractional coverage (unitless). See tables S5, S7. 30, 32

$f_{a}$ fraction of the tile which is saturated and hence has aerodynamic resistance only (unitless). This represents 1 for lake, ice or snow-covered tiles (unitless). See tables S7, S24. 32, 49

$f_{a h}$ roughness length of the surface beneath the canopy (unitless). See table S11. 36

$f_{b u}$ fraction of the built-up areas (unitless). See tables S18, S20, S25. 43, 45, 50 
$f_{B_{P}}$ fraction of the area that receives precipitation rate greater than or equal to precipitation rate (unitless). See table S6. 31

$f_{c a}$ fraction of the vegetation or canopy class in a grid cell (unitless). See tables S4, S5, S23, S24, S50, S52. 29, 30, 48, 49, 75,77

$f_{c a, e x}$ fraction of the exposed canopy (unitless). See table $\mathbf{S} 48.73$

$f_{c a, G}$ fraction of the vegetation in a grid cell. See table S24. 49

$f_{c a, \max }$ maximum fraction of vegetation type including non-biological fraction (unitless). See table S5. 30

$f_{c a, n}$ vegetation fractional coverage for the $n$ vegetation tile. See table S1. 26

$f_{c a, s n}$ canopy wetness-snow cover fraction (unitless). See tables S7, S11. 32, 36

$f_{c a, w e t}$ fractional wetted area of the canopy (unitless). See table S23. 48

$f_{\text {cons }, A}$ ratio of consumption to abstraction (unitless). See tables S54, S55, S57, S58, S60, S61, S63, S64, S70, S71, S73, S74, S82, S83. 79, 80, 82, 83, 85, 86, 88, 89, 95, 96, 98, 99, 107, 108

$f_{\text {day,ca,wet }}$ fraction of the day-time when the canopy is wet (unitless). See table S23. 48

$260 f_{\text {drai }}$ drainage decay factor $\left(\mathrm{L}^{-1}\right)$. See table $\mathrm{S} 31.56$

$f_{F}$ frozen ration in the uppermost soil layer (unitless). See table S11. 36

$f_{g w, u s e}$ sector- and cell-specific groundwater use fraction (unitless). See tables S53, S54, S56, S57, S59, S60, S61, S62, S63, S64, S69, S70, S72, S73. 78, 79, 81, 82, 84-89, 94-99

$f_{G, s a t}$ fraction of the grid area which is saturated (unitless). It is determined by the topographic characteristics and soil moisture state of a grid cell. See tables S22, S58. 47, 83

$f_{G, \text { unsat }}$ fraction of the grid area which is unsaturated (unitless). See table S21. 46

$f_{h 2 o}$ fraction of the ground covered by water (unitless). See tables S21, S25. 46, 50

$f_{h g}$ hydrogeology-related factor (unitless). See table S30. 55

$f_{i f}$ factor interflow (unitless). See table S26. 51

$270 f_{i r r}$ storage reduction factor due to irrigation water abstraction (unitless). See tables S29, S39, S43, S52, S53, S56. 54, 64, 68, $77,78,81$

$f_{\text {irr }, \text { eff }}$ irrigation efficieny (unitless). See tables S56, S58. 81, 83

$f_{i r r, s w, \text { eff }}$ country-specific surface water irrigation efficieny (unitless). See table S56. 81

$f_{l a}$ lake area fraction of the grid cell (unitless). See table S49. 74

$275 f_{\text {lost }}$ proportion lost during delivery (unitless). See tables S55, S57, S58, S60, S61, S63, S64, S70, S73, S71, S74, S83. 80, 82, $83,85,86,88,89,95,96,98,99,108$

$f_{L A I}$ interception efficiency of leaf area index (unitless). See tables S4, S9. 29, 34

$f_{p g}$ permafrost / glacier-related factor (unitless). See table S30. 55

$f_{P}$ percentage that receives a precipitation rate greater than or equal to precipitation rate over the fractions of a grid cell (unitless). See table S6. 31 
$f_{P c, G}$ fraction of the grid cell occupied by convective precipitation (unitless). See tables S5, S20. 30, 45

$f_{r e}$ fraction of the reservoir fill equal to 1 at total storage capacity (unitless). See table S37. 62

$f_{r}$ relief-related factor (unitless). See table S30. 55

$f_{r i, s a t}$ river channel fraction in the saturated area (unitless). See table S22. 47

$285 f_{\text {root }, i}$ fraction of the roots per soil layer $i$ (unitless). See table S24. 49

$f_{R}$ runoff component factor (surface, interflow, baseflow) (unitless). See table S19. 44

$f_{R_{i f, \text { max }}}$ fraction of the maximum interflow or subsurface flow (unitless). See table S31. 42

$f_{R_{i n, 0}}$ fraction of the area for which the maximum rate of infiltration (infiltration capacity) is less than rate of the infiltration (unitless). See table S24. 49

$290 f_{s n}$ fraction of the ground covered by snow (unitless). See tables S7, S8, S10, S11, S12, S25. 32, 33, 35-37, 50

$f_{\text {so,bare }}$ fraction of the bare soil in a grid cell (unitless). See tables S1, S24. 26, 49

$f_{\text {so,bare,sat }}$ fraction of the bare soil in a grid cell that is saturated (unitless). See table S24. 49

$f_{\text {so,dep }}$ soil depletion fraction (unitless). See table S23. 48

$f_{\text {so,tex }}$ soil-texture-related factor (unitless). See table S30. 55

$f_{s u, g r}$ fractional area of surface ground (unitless). See table S24. 49

$f_{s w b}$ fraction of the surface water bodies (lakes, wetlands, reservoirs) (unitless). See tables S32, S43. 57, 68

$f_{S_{s o, m a x}}$ fraction of the maximum soil moisture (unitless). See table S31. 42

$f_{w, l f}$ coverage of the water on leaf (unitless). See table S7. 32

$f_{w e}$ fraction coverage of wetland in a grid cell (unitless). See tables S20, S25, S27, S39, S40, S41. 45, 50, 52, 55, 64-66

$300 F$ mass gain due to frost $\left(\mathrm{L}^{3} \mathrm{~L}^{-2} \mathrm{~T}^{-1}\right)$. See table $\mathrm{S} 8, \mathrm{~S} 13.33,38$

$F_{\text {so }}$ frozen soil $(\Theta)$. See table S13. 38

$g_{c a}$ canopy conductance $\left(\mathrm{M} \mathrm{L} \mathrm{T}^{-3} \Theta^{-1}\right)$. See table S23. 48

$g_{c a}^{a i r}$ water vapor conductance from the canopy air to the atmosphere $\left(\mathrm{M} \mathrm{L} \mathrm{T}^{-3} \Theta^{-1}\right)$. See table S23. 48

$g_{c a}^{H_{s}}$ vegetation sensible heat conductance $\left(\mathrm{M} \mathrm{L} \mathrm{T}^{-3} \Theta^{-1}\right)$. See table S23. 48

$g_{g r}^{c a}$ water vapor conductance from ground to canopy air $\left(\mathrm{M} \mathrm{L} \mathrm{T}^{-3} \Theta^{-1}\right)$. See table S23. 48

$g_{\text {so }}$ soil conductance $\left(\mathrm{M} \mathrm{L} \mathrm{T} \mathrm{T}^{-3} \Theta^{-1}\right)$. See table S24. 49

$g_{s t}^{w}$ water vapor leaf level stomatal conductance (M L T $\left.\mathrm{T}^{-3} \Theta^{-1}\right)$. See tables S7, S23. 32, 48

$g_{s t, p o t}$ potential leaf level stomatal conductance, non-water stressed $\left(\mathrm{M} \mathrm{L} \mathrm{T}^{-3} \Theta^{-1}\right)$. See table S23. 48

$G$ grid cell (1). See table S49. 74

$310 G A V$ gross added value per country (money $\mathrm{T}^{-1}$ ). See tables $\mathrm{S} 69, \mathrm{~S} 70, \mathrm{~S} 72, \mathrm{~S} 73.94,95,97,98$ 
$h_{\text {of }}$ overflow height (L). See tables S34, S37. 59, 62

$h_{w}$ water height (L). See tables S34, S37. 59, 62

$h_{w, w e}$ wetland water level (L ). See table S42. 67

$H$ heat $\left(\mathrm{M} \mathrm{L}^{2} \mathrm{~T}^{-2}\right)$. See table S12. 37

$315 H_{a t m}$ heat flux from the overlaying atmosphere $\left(\mathrm{M} \mathrm{L}^{2} \mathrm{~T}^{-3} \mathrm{~L}^{-2}\right)$. See table S13. 38

$H_{i}$ excess or deficit of energy needed to change the soil layer temperature to freezing temperature ( $\mathrm{M} \mathrm{T}^{-2}$ ). See table S12. 37

$H_{l}$ latent heat $\left(\mathrm{M} \mathrm{T}^{-3}\right)$. See tables S2, S7, S8, S10, S12. 27, 32, 33, 35, 37

$H_{l, E}$ latent heat of evaporation ( $\mathrm{M} \mathrm{T}^{-3}$ ). See tables S7, S8, S9, S11, S23, S24. 27, 32, 33, 36, 48, 49

$H_{l, E_{s n}}$ latent heat of sublimation $\left(\mathrm{M} \mathrm{T}^{-3}\right)$. See tables S7, S11. 32, 36

$H_{M}$ latent heat of melt $\left(\mathrm{M} \mathrm{T}^{-3}\right)$. See tables S8, S12.33, 37

$H_{M, i c e}$ latent heat of ice $\left(\mathrm{M} \mathrm{T}^{-3}\right)$. See table S12. 37

$H_{\text {se }}$ sensible heat flux $\left(\mathrm{M} \mathrm{T}^{-3}\right)$. See tables S10,S12. 35, 37

$H_{s n}$ snow heat content $\left(\mathrm{M} \mathrm{T}^{-3}\right)$. See table $\mathrm{S} 12.37$

$H_{s n, i}$ snow heat content of layer $i\left(\mathrm{M} \mathrm{T}^{-3}\right)$. See table $\mathrm{S} 12.37$

$H_{s o}$ soil heat flux density $\left(\mathrm{M} \mathrm{T}^{-3}\right)$. See table S2. 27

$H_{\text {soTL }}$ reciprocal areal heat capacity of the top soil layer $\left(\mathrm{M} \mathrm{T}^{-3}\right)$. See table S12. 37

$H_{s o, i}$ specific heat capacity of layer $i\left(\mathrm{M} \mathrm{L}^{2} \mathrm{~T}^{-2}\right)$. See table S13. 38

$H_{t o t}$ total surface heat capacity $\left(\mathrm{M} \mathrm{L}^{2} \mathrm{~T}^{-2}\right)$. See table S12. 37

$i$ number of element or layer or space index (unitless). See tables S8, S12, S19. 33, 37, 44

$i_{G S}$ growing-season index (unitless). See table S7. 32

$I_{0}$ initial intercepted canopy snow load (unitless). See table S9. 34

$I_{\text {cap }}$ interception capacity (L). See table S3. 29

$j$ weighting parameter that varies between 0 and 1. See table S52. 77

$J_{\text {ele, } A, \text { ints }, i}$ water abstraction intensity of powerplant $i$. See table S75. 100

$J_{\text {ele,cons, }, \text { ints }, i}$ water consumption intensity of powerplant $i$. See table S76. 101

$J_{\text {ele }, \text { coo, } i}$ cooling system of powerplant $i$. See tables S75, S76. 100, 101

$J_{\text {ele }, p t, i}$ plant type of powerplant $i$. See tables S75, S76. 100, 101

$J_{\text {ele,prod, } i}$ thermal electricity production of powerplant $i\left(\mathrm{MWh} \mathrm{T}^{-1}\right)$. See tables S75, S76. 100, 101

$k$ soil hydraulic conductivity $\left(\mathrm{L} \mathrm{T}^{-1}\right)$. See tables S13, S14, S21, S25, S27, S28. 38, 39, 46, 50, 52, 53

$k_{3 \text { so }}$ hydraulic conductivity from the third soil layer to the deeper soil $\left(\mathrm{L} \mathrm{T}^{-1}\right)$. See tables S27, S28, S30. 52, 53, 55 
$k_{a q}$ hydraulic conductivity of the layer containing the water table (unitless). See table S30. 55

$k_{b o t}$ hydraulic conductivity of the bottom soil with free gravitational drainage $\left(\mathrm{L} \mathrm{T}^{-1}\right)$. See table $\mathrm{S} 31.56$

$k_{j}$ hydraulic conductivity at the interface $j$ (unitless). See table S25. 50

$k_{b}$ recession coefficient of the saturated/groundwater storage, producing baseflow /groundwater runoff $\left(\mathrm{T}^{-1}\right)$. See table S31. 56

$k_{0}$ recession coefficient of the unsaturated zone storage, upper outlet for fast interflow $\left(\mathrm{T}^{-1}\right)$. See table S26. 51

$k_{1}$ recession coefficient of the unsaturated zone reservoir, lower outlet for slow interflow $\left(\mathrm{T}^{-1}\right)$. See table S26. 51

$k_{u z}$ recession coefficient of the unsaturated zone reservoir to the saturated/groundwater reservoir (groundwater recharge / percolation ) $\left(\mathrm{T}^{-1}\right)$. See table S30. 55

$k_{\text {sat }}$ effective saturated hydraulic conductivity (unitless). See tables S13, S25, S28. 38, 50, 53

$k_{\text {sat }}^{g w}$ saturated hydraulic conductivity for groundwater (unitless). See table S31. 56

$k_{t h}$ thermal conductivity (unitless). See table S13.38

$K_{f}$ generatio ratio for interflow (unitless). See table S26. 51

$K_{f f}$ generatio ratio for overflow of the uppermost layer, i.e. the Horton runoff (unitless). See table S22. 47

$l_{G}$ distance between centers of neighboring grid cells (L). See table S46. 71

$l_{G, r i}$ distance between centers of neighboring grid cells, in river flow direction (L). See table S42. 67

$l_{r i}$ length of river sections (L). See tables S45, S46. 24, 70, 71

$l_{\text {ri,hom }}$ length of homogeneous rivers segments (L). See table S46. 71

$L A I$ leaf area index $\left(\mathrm{L} \mathrm{L}^{-1}\right)$. See tables S4, S5, S6, S7, S9, S49. 29-32, 34, 73-76

$L A I_{\text {max }}$ maximum leaf area index $\left(\mathrm{L} \mathrm{L}^{-1}\right)$. See table $\mathrm{S} 7.32$

$L A I_{\text {min }}$ minimum leaf area index $\left(\mathrm{L} \mathrm{L}^{-1}\right)$. See table $\mathrm{S} 7.32$

$L A I_{\text {month }}$ leaf area index of monthly vegetation (unitless). See table S7. 32

$L C$ land cover (unitless). See table S19. 44

$M$ snowmelt, water that changes from solid to liquid $\left(\mathrm{L}^{3} \mathrm{~L}^{-2} \mathrm{~T}^{-1}\right)$. See tables S8, S11, S12, S14, S20, S21, S25, S39. 33, 36, $37,39,45,46,50,64$

$M_{c a}$ snowmelt on canopy $\left(\mathrm{L}^{3} \mathrm{~L}^{-2} \mathrm{~T}^{-1}\right)$. See table S9. 34

$M_{i}$ snowmelt of layer $i\left(\mathrm{~L}^{3} \mathrm{~L}^{-2} \mathrm{~T}^{-1}\right)$. See table $\mathrm{S} 12.37$

$M_{\text {in }}$ flow of liquid water into layer $i$ from the layer above $\left(\mathrm{L}^{3} \mathrm{~L}^{-2} \mathrm{~T}^{-1}\right)$. See table S8. 33

$M_{\text {out }}$ flow of liquid water out of layer $i$ to the layer below $\left(\mathrm{L}^{3} \mathrm{~L}^{-2} \mathrm{~T}^{-1}\right)$. See table S8. 33

$370 M_{p o t}$ potential snowmelt, amount of water that could change from solid to liquid independent of snow storage state $\left(\mathrm{L}^{3} \mathrm{~L}^{-2} \mathrm{~T}^{-1}\right)$. See tables S8, S12. 33, 37 
$M_{S G_{i}}$ snowmelt in a subgrid cell Müller Schmied et al. [34] $\left(\mathrm{L}^{3} \mathrm{~L}^{-2} \mathrm{~T}^{-1}\right)$. See table S8. 33

$M_{t}$ snowmelt at the time $t\left(\mathrm{~L}^{3} \mathrm{~L}^{-2} \mathrm{~T}^{-1}\right)$. See table $\mathrm{S} 12.37$

$M_{\text {underca }}$ snowmelt under canopy $\left(\mathrm{L}^{3} \mathrm{~L}^{-2} \mathrm{~T}^{-1}\right)$. See table S10. 35

$375 n$ linear reservoir cascade index (unitless). See table S46. 71

$N R$ net radiation flux $\left(\mathrm{M} \mathrm{L}^{-2} \mathrm{~T}^{-3}\right)$. See tables $\mathrm{S} 2, \mathrm{~S} 10, \mathrm{~S} 12.27,35,37$

$N R_{n}$ net radiation at crop surface $\left(\mathrm{M} \mathrm{J} \mathrm{L}^{-2} \mathrm{~T}^{-1}\right)$. See table $\mathrm{S} 2.27$

$P_{\text {conv }}$ relative amount of convective precipitation $\left(\mathrm{L}^{3} \mathrm{~L}^{-2} \mathrm{~T}^{-1}\right)$. See table $\mathrm{S} 21.46$

$P_{d r}$ drip, dripping of water at the edge of the canopy $\left(\mathrm{L}^{3} \mathrm{~L}^{-2} \mathrm{~T}^{-1}\right)$. See tables S5, S9. 30, 34

$380 P_{d r, l}$ dripping of rain at the edge of the canopy $\left(\mathrm{L}^{3} \mathrm{~L}^{-2} \mathrm{~T}^{-1}\right)$. See table S5. 30

$P_{d r, s}$ dripping of snow at the edge of the canopy $\left(\mathrm{L}^{3} \mathrm{~L}^{-2} \mathrm{~T}^{-1}\right)$. See tables S5, S9. 30, 34

$P_{\text {eff }}$ effective precipitation reaching the soil surface $\left(\mathrm{L}^{3} \mathrm{~L}^{-2} \mathrm{~T}^{-1}\right)$. See table $\mathrm{S} 57.82$

$P_{g r}$ total precipitation falls directly to the ground (snow or soil surface) $\left(\mathrm{L}^{3} \mathrm{~L}^{-2} \mathrm{~T}^{-1}\right)$. See table $\mathrm{S} 6.31$

$P_{g r, l}$ rainfall falls directly to the ground $\left(\mathrm{L}^{3} \mathrm{~L}^{-2} \mathrm{~T}^{-1}\right)$. See table S6. 31

$P_{g r, l, c a l m}$ rainfall falling to the ground in calm areas $\left(\mathrm{L}^{3} \mathrm{~L}^{-2} \mathrm{~T}^{-1}\right)$. See table S4. 29

$P_{g r, l, s t o r m y}$ rainfall falling to the ground in stormy areas $\left(\mathrm{L}^{3} \mathrm{~L}^{-2} \mathrm{~T}^{-1}\right)$. See table S4. 29

$P_{g r, s}$ snowfall falls directly to the ground $\left(\mathrm{L}^{3} \mathrm{~L}^{-2} \mathrm{~T}^{-1}\right)$. See tables S6, S10. 31, 35

$P_{g r, s, c a l m}$ snowfall falling to the ground in calm areas $\left(\mathrm{L}^{3} \mathrm{~L}^{-2} \mathrm{~T}^{-1}\right)$. See table S4. 29

$P_{g r, s, s t o r m y}$ snowfall falling to the ground in stormy areas $\left(\mathrm{L}^{3} \mathrm{~L}^{-2} \mathrm{~T}^{-1}\right)$. See table S4. 29

$P_{\text {int }}$ precipitation intercepted by canopy $\left(\mathrm{L}^{3} \mathrm{~L}^{-2} \mathrm{~T}^{-1}\right)$. See tables $\mathrm{S} 4, \mathrm{~S} 7, \mathrm{~S} 3.28-30,32$

$P_{\text {int }, l}$ interception of rainfall by canopy $\left(\mathrm{L}^{3} \mathrm{~L}^{-2} \mathrm{~T}^{-1}\right)$. See tables $\mathrm{S} 3, \mathrm{~S} 4, \mathrm{~S} 5, \mathrm{~S} 10.28-30,35$

$P_{\text {int }, l, c a l m}$ interception of rainfall by canopy in calm areas $\left(\mathrm{L}^{3} \mathrm{~L}^{-2} \mathrm{~T}^{-1}\right)$. See table S4. 29

$P_{\text {int }, l, \text { stormy }}$ interception of rainfall by canopy in stormy areas $\left(\mathrm{L}^{3} \mathrm{~L}^{-2} \mathrm{~T}^{-1}\right)$. See table S4. 29

$P_{\text {int, } \max }$ calibration parameter of the canopy storage (L). See table S49. 75

$P_{\text {int }, s}$ interception of snowfall by canopy $\left(\mathrm{L}^{3} \mathrm{~L}^{-2} \mathrm{~T}^{-1}\right)$. See tables S3, S4, S5, S9. 28-30, 34

$P_{\text {int }, s, \text { calm }}$ interception of snowfall by canopy in calm areas $\left(\mathrm{L}^{3} \mathrm{~L}^{-2} \mathrm{~T}^{-1}\right)$. See table S4. 29

$P_{\text {int }, s, s t o r m y}$ interception of snowfall by canopy in stormy areas $\left(\mathrm{L}^{3} \mathrm{~L}^{-2} \mathrm{~T}^{-1}\right)$. See table $\mathrm{S} 4.29$

$P_{r a}$ rainfall $\left(\mathrm{L}^{3} \mathrm{~L}^{-2} \mathrm{~T}^{-1}\right)$. See tables $\mathrm{S} 4, \mathrm{~S} 5, \mathrm{~S} 6, \mathrm{~S} 8, \mathrm{~S} 9, \mathrm{~S} 12$, S20, S25, S39. 29-31, 33, 37, 45, 50, 64

$P_{\text {mean }, G}$ grid cell average precipitation $\left(\mathrm{L}^{3} \mathrm{~L}^{-2} \mathrm{~T}^{-1}\right)$. See tables S5, S6. 30, 31

$400 P_{s n}$ snowfall $\left(\mathrm{L}^{3} \mathrm{~L}^{-2} \mathrm{~T}^{-1}\right)$. See tables $\mathrm{S} 4, \mathrm{~S} 5, \mathrm{~S} 6, \mathrm{~S} 9, \mathrm{~S} 12.29-31,33,34$

$P_{s, c a}$ snowfall that is affected by the canopy interception and dripping $\left(\mathrm{L}^{3} \mathrm{~L}^{-2} \mathrm{~T}^{-1}\right)$. See table $\mathrm{S} 8.33$ 
$P_{s, S G_{i}}$ snowfall in a subgrid (SG) cell Müller Schmied et al. [34] $\left(\mathrm{L}^{3} \mathrm{~L}^{-2} \mathrm{~T}^{-1}\right)$. See table S8. 33

$P_{t h}$ throughfall, total precipitation falls to the ground through canopy spaces $\left(\mathrm{L}^{3} \mathrm{~L}^{-2} \mathrm{~T}^{-1}\right)$. See tables $\mathrm{S} 3, \mathrm{~S} 5, \mathrm{~S} 8, \mathrm{~S} 9, \mathrm{~S} 12$, S14, S20, S21, S22, S25, S30. 28, 30, 33, 34, 37, 39, 45-47, 50, 55

405

$P E T_{\text {ows }}$ total amount of water from transpiration, evaporation from the impervious areas, defined here as open water storage $\left(\mathrm{L}^{3} \mathrm{~L}^{-2} \mathrm{~T}^{-1}\right)$. See tables S20, S24. 45, 49

PFT plant functional type (unitless). See tables S48, S49. 73, 74

POP population within the gridcell. See tables S59, S62. 84, 87

$P O P_{l i v, t}$ livestock type specific animal population within the gridcell. See table S68. 93

$\mathrm{POP}_{u}$ urban population within the gridcell. See tables S69, S70, S71, S72, S73, S74. 94-99

$P O P_{u}^{c}$ urban population of a country. See tables S69, S70, S71, S72, S73, S74. 94-99

$q$ specific humidity of near-surface air $\left(\mathrm{M} \mathrm{M}^{-1}\right)$. See tables S2, S7, S11, S23, S24. 27, 32, 36, 48, 49

$q_{g r}$ specific humidity at ground surface $\left(\mathrm{M} \mathrm{M}^{-1}\right)$. See tables S2, S23. 27, 48

$q_{\text {sat }}^{c a}$ saturated specific humidity at canopy temperature $\left(\mathrm{M} \mathrm{M}^{-1}\right)$. See tables S7, S11, S23, S24. 32, 36, 48, 49

$q_{s a t}^{g r}$ saturated specific humidity at ground surface $\left(\mathrm{M} \mathrm{M}^{-1}\right)$. See tables S2, S11, S24. 27, 36, 49

$q_{\text {sat }}^{g r, s n}$ saturated specific humidity at ground surface with snow $\left(\mathrm{M} \mathrm{M}^{-1}\right)$. See table S11. 36

$q_{\text {sat }}^{\text {sn }}$ saturated specific humidity at snow $\left(\mathrm{M} \mathrm{M}^{-1}\right)$. See table S7. 32

$q_{\text {so }}$ relative humidity of the soil pore space $\left(\mathrm{M} \mathrm{M}^{-1}\right)$. See table $\mathrm{S} 24.49$

$q_{s u}$ relative humidity at the near-surface $\left(\mathrm{M} \mathrm{M}^{-1}\right)$ dimensions. See table S11. 36

$Q_{e f}$ environmental flow $\left(\mathrm{L}^{3} \mathrm{~T}^{-1}\right)$. See tables S35, S56, S62, S72, S75. 60

$Q_{i}$ vertical water flux from soil layer above, including infiltration in the upper layer and percolation and capillary rise in all layers $\left(\mathrm{L}^{3} \mathrm{~T}^{-1}\right)$. See table S14. 39

$Q_{i-1}$ vertical water flux to soil layer below, including infiltration in the upper layer and percolation and capillary rise in all layers $\left(\mathrm{L}^{3} \mathrm{~T}^{-1}\right)$. See table $\mathrm{S} 14.39$ 
$Q_{\text {in,so }}$ soil moisture flux $\left(\mathrm{L}^{3} \mathrm{~T}^{-1}\right)$. See table S13. 38

$Q_{\text {in,surf }}$ surface moisture flux remaining after surface runoff has been removed $\left(\mathrm{L}^{3} \mathrm{~T}^{-1}\right)$. See table $\mathrm{S} 25.50$

$Q_{i u}$ inflow upstream of a grid cell $\left(\mathrm{L}^{3} \mathrm{~T}^{-1}\right)$. See tables S32, S35, S43, S45, S46, S54, S55, S56, S57, S59, S60, S61, S62, S63, S65, S66, S67, S68, S69, S70, S71, S72, S73. 57, 60, 68, 70, 71, 79-82, 84-88, 90-98

$440 Q_{i u, l a}$ mean total annual inflow in a lake $\left(\mathrm{L}^{3} \mathrm{~T}^{-1}\right)$. See tables $\mathrm{S} 34, \mathrm{~S} 43.59,68$

$Q_{\text {iu, mean }}$ mean annual inflow in a reservoir $\left(\mathrm{L}^{3} \mathrm{~T}^{-1}\right)$. See table $\mathrm{S} 37.62$

$Q_{\text {iu,re }}$ inflow reservoir $\left(\mathrm{L}^{3} \mathrm{~T}^{-1}\right)$. See tables S35, S37, S43. 60, 62, 68

$Q_{i u, t o t, r e}$ mean total annual inflow in a reservoir $\left(\mathrm{L}^{3} \mathrm{~T}^{-1}\right)$. See table S37. 62

$Q_{i u, w e, u p}$ inflow from the wetland of an upstream grid cell $\left(\mathrm{L}^{3} \mathrm{~T}^{-1}\right)$. See tables S39, S40, S45. 64, 65, 70

$445 Q_{l a}$ outflow from a lake $\left(\mathrm{L}^{3} \mathrm{~T}^{-1}\right)$. See tables S32, S34, S43. 57, 59, 60

$Q_{\text {la,global }}$ outflow from a global lake $\left(\mathrm{L}^{3} \mathrm{~T}^{-1}\right)$. See tables S32, S34, S39. 57, 59, 64

$Q_{\text {la,local }}$ outflow from a local lake $\left(\mathrm{L}^{3} \mathrm{~T}^{-1}\right)$. See tables S32, S34, S39. 57, 59, 64

$Q_{\text {mean }}$ outflow mean $\left(\mathrm{L}^{3} \mathrm{~T}^{-1}\right)$. See table S35. 60

$Q_{o d}$ outflow downstream of a grid cell $\left(\mathrm{L}^{3}\right)$. See table S43. 68

$Q_{r v, \text { up }}$ outflow from rivulet storage of upstream grid cells $\left(\mathrm{L}^{3} \mathrm{~T}^{-1}\right)$. See tables S40, S45. 65, 70

$Q_{p f}$ preferential flow $\left(\mathrm{L}^{3} \mathrm{~T}^{-1}\right)$. See tables $\mathrm{S} 21, \mathrm{~S} 29, \mathrm{~S} 30.46,54,55$

$Q_{r e}$ outflow from a local reservoir that flows directly into the river channel of the cell $\left(\mathrm{L}^{3} \mathrm{~T}^{-1}\right)$. See tables S35, S37, S39, S43, S46. 60, 62, 64, 68, 71

$Q_{r e, d e}^{i r r}$ outflow from a irrigation reservoir driven by water demand in downstream cells $\left(\mathrm{L}^{3} \mathrm{~T}^{-1}\right)$. See table S37. 62

$455 Q_{\text {re,global }}$ outflow from a global reservoir $\left(\mathrm{L}^{3} \mathrm{~T}^{-1}\right)$. See table S37. 62

$Q_{r e, g l o b a l}^{i r r}$ outflow from a global reservoir designed for irrigation $\left(\mathrm{L}^{3} \mathrm{~T}^{-1}\right)$. See table S37. 62

$Q_{r e, g l o b a l}^{\text {non-irr }}$ outflow from a global reservoir designed for other purposes than irrigation $\left(\mathrm{L}^{3} \mathrm{~T}^{-1}\right)$. See table S37. 62

$Q_{\text {re,global }}^{\text {purpose }}$ outflow from a global reservoir for irrigation or others purposes $\left(\mathrm{L}^{3} \mathrm{~T}^{-1}\right)$. See table S37. 62

$Q_{\text {re,mean }}$ long-term mean outflow from a reservoir $\left(\mathrm{L}^{3} \mathrm{~T}^{-1}\right)$. See table S37. 62

$460 Q_{r e, \min }$ minimum outflow from reservoir $\left(\mathrm{L}^{3} \mathrm{~T}^{-1}\right)$. See table S37. 62

$Q_{r e, n d}$ non-damaging outflow from reservoir $\left(\mathrm{L}^{3} \mathrm{~T}^{-1}\right)$. See table S37. 62

$Q_{\text {re,norm }}$ normal outflow from reservoir $\left(\mathrm{L}^{3} \mathrm{~T}^{-1}\right)$. See table S37. 62

$Q_{\text {re,local }}$ outflow from a local reservoir $\left(\mathrm{L}^{3} \mathrm{~T}^{-1}\right)$. See tables S43, S37, S46. 60, 62, 71

$Q_{r i}$ streamflow $\left(\mathrm{L}^{3}\right)$. See tables S35, S43, S45, S46. 60, 68, 70, 71

$Q_{r i, \text { in }}$ streamflow inflow $\left(\mathrm{L}^{3} \mathrm{~T}^{-1}\right)$. See tables S32, S35, S46. 57, 60, 71 
$Q_{r i, n}$ outflow of river storage cascade $n\left(\mathrm{~L}^{3} \mathrm{~T}^{-1}\right)$. See table $\mathrm{S} 43.68$

$Q_{r i, n-1}$ outflow of prior river storage cascade $n-1\left(\mathrm{~L}^{3} \mathrm{~T}^{-1}\right)$. See table S43. 68

$Q_{\text {ri,out }}$ streamflow outflow $\left(\mathrm{L}^{3} \mathrm{~T}^{-1}\right)$. See tables S32, S35, S46. 57, 60, 71

$Q_{r i, u p}$ streamflow from the upstream grid cell $\left(\mathrm{L}^{3} \mathrm{~T}^{-1}\right)$. See tables $\mathrm{S} 40, \mathrm{~S} 45.65,70$

$Q_{w e}$ outflow from wetland $\left(\mathrm{L}^{3} \mathrm{~T}^{-1}\right)$. See tables S39, S42. 64, 67

$Q_{\text {we, global }}$ outflow from a global wetland $\left(\mathrm{L}^{3} \mathrm{~T}^{-1}\right)$. See tables S39, S42, S43. 64, 67, 68

$Q_{\text {we }, \text { local }}$ outflow from a local wetland $\left(\mathrm{L}^{3} \mathrm{~T}^{-1}\right)$. See tables S32, S35, S39, S42. 57, 60, 64, 67

$r_{b}$ bulk canopy resistance $\left(\mathrm{T} \mathrm{L}^{-1}\right)$. See tables S7, S11, S23. 32, 36, 48

$r_{c a}$ vegetation or canopy aerodynamic resistance $\left(\mathrm{T} \mathrm{L}^{-1}\right.$; for Mac-PDM.20 $\mathrm{cms}^{-1}$ ). See tables S2, S7, S11, S23. 27, 32, 36, 48

$r_{c a, d r y}$ aerodynamic resistance of the dry leaves $\left(\mathrm{T} \mathrm{L}^{-1}\right)$. See table S23. 48

$r_{\text {floodplain }}$ floodplain resistance (unitless). See tables S11, S24. 36, 49

$r_{o}$ architectural resistance $\left(\mathrm{T} \mathrm{L}^{-1}\right)$. See tables S7, S23. 32, 48

$r_{s n}$ snow resistance (unitless). See tables S7, S11, S23, S24. 32, 36, 48, 49

$r_{\text {so }}$ bare soil resistance $\left(\mathrm{T} \mathrm{L}^{-1}\right)$. See tables S11, S24. 36, 49

$r_{t o t}$ total resistance to water vapor transfer from the canopy to the canopy air $\left(\mathrm{T} \mathrm{L}^{-1}\right)$. See table S7. 32

$r_{w}$ aerodynamic resistance ( $\mathrm{T} \mathrm{L}^{-1}$; for Mac-PDM. $20 \mathrm{cms}^{-1}$ ). See tables S2, S7, S23. 27, 32, 48

$r_{w c a}$ aerodynamic resistance under canopy air space $\left(\mathrm{T} \mathrm{L}^{-1}\right)$. See tables S11, S24. 49

$R_{0}$ runoff $0\left(\mathrm{~L}^{3} \mathrm{~L}^{-2} \mathrm{~T}^{-1}\right)$. See table S20. 45

$R_{1}$ runoff $1\left(\mathrm{~L}^{3} \mathrm{~L}^{-2} \mathrm{~T}^{-1}\right)$. See table S20. 45

$R_{2}$ runoff $2\left(\mathrm{~L}^{3} \mathrm{~L}^{-2} \mathrm{~T}^{-1}\right)$. See table $\mathrm{S} 20.45$

$R_{b u}$ immediate runoff in urban areas $\left(\mathrm{L}^{3} \mathrm{~L}^{-2} \mathrm{~T}^{-1}\right)$. See table S18. 43

$R_{c r}$ capillary rise $\left(\mathrm{L}^{3} \mathrm{~L}^{-2} \mathrm{~T}^{-1}\right)$. See tables $\mathrm{S} 14, \mathrm{~S} 26, \mathrm{~S} 28, \mathrm{~S} 29, \mathrm{~S} 30.39,51,53-55$

$R_{c r, \max }$ maximum capillary rise in a cell fraction, depending on height of ground water table and relative elevation of grid $\left(\mathrm{L}^{3} \mathrm{~L}^{-2} \mathrm{~T}^{-1}\right)$. See table S28. 53

$R_{G}$ total runoff of a grid (G) cell, a lag process between runoff generation and river routing for each grid cell $\left(\mathrm{L}^{3} \mathrm{~L}^{-2} \mathrm{~T}^{-1}\right)$. See table S19, S44. 44, 69

$R_{g l, w e, l a}$ liquid runoff from glaciers, wetlands, and lakes / is this a part of saturation excess flow? $\left(\mathrm{M} \mathrm{L}^{-2} \mathrm{~T}^{-1}\right)$. See table S20. 45

$495 R_{g w}$ groundwater runoff, outflow of the groundwater storage $\left(\mathrm{L}^{3} \mathrm{~L}^{-2} \mathrm{~T}^{-1}\right)$. See tables $\mathrm{S} 14, \mathrm{~S} 29, \mathrm{~S} 30, \mathrm{~S} 31, \mathrm{~S} 32, \mathrm{~S} 43, \mathrm{~S} 54$, S55, S56, S57, S59, S60, S61, S62, S63, S65, S66, S67, S68, S69, S70, S71, S72, S73. 39, 42, 43, 54-57, 68, 79-82, 84-88, 90-98 
$R_{g w}^{r i}$ groundwater runoff which recharges rivers $\left(\mathrm{L}^{3} \mathrm{~L}^{-2} \mathrm{~T}^{-1}\right)$. See table $\mathrm{S} 43.68$

$R_{g w, \max }$ Maximum drainage when the water table depth is at the surface $\left(\mathrm{L}^{3} \mathrm{~L}^{-2} \mathrm{~T}^{-1}\right)$. See table $\mathrm{S} 31.56$

$R_{\text {gw,rout }}$ groundwater routing parameter for Mac-PDM.20 (unitless). See table S31. 56

$R_{g w, u p}$ groundwater runoff from the upstream grid cell $\left(\mathrm{L}^{3} \mathrm{~L}^{-2} \mathrm{~T}^{-1}\right)$. See tables $\mathrm{S} 40, \mathrm{~S} 45.65,70$

$R_{\text {gwr }}$ groundwater recharge $\left(\mathrm{L}^{3} \mathrm{~L}^{-2} \mathrm{~T}^{-1}\right)$. See tables S14, S20, S29, S30, S54. 39, 42, 45, 54, 55

$R_{\text {gwr }}^{\text {swb }}$ groundwater recharge below surface water bodies $\left(\mathrm{L}^{3} \mathrm{~L}^{-2} \mathrm{~T}^{-1}\right)$. See tables S32, S35, S39. 57, 60, 64

$R_{g w r, i}$ groundwater recharge in layer $i\left(\mathrm{~L}^{3} \mathrm{~L}^{-2} \mathrm{~T}^{-1}\right)$. See table $\mathrm{S} 14.39$

$R_{g w r, i-1}$ groundwater recharge in layer $i-1\left(\mathrm{~L}^{3} \mathrm{~L}^{-2} \mathrm{~T}^{-1}\right)$. See table S14. 39

$R_{\text {gwr,max }}$ maximum groundwater recharge $\left(\mathrm{L}^{3} \mathrm{~L}^{-2} \mathrm{~T}^{-1}\right)$. See tables $\mathrm{S} 30, \mathrm{~S} 39.55,64$

$R_{\text {gwr,min }}$ minimum groundwater recharge $\left(\mathrm{L}^{3} \mathrm{~L}^{-2} \mathrm{~T}^{-1}\right)$. See table $\mathrm{S} 27.55$

$R_{G}^{n}$ runoff concentration in a grid $(\mathrm{G})$ cell, of a time step $n$, represents the lag process between runoff generation and river routing, for each grid cell. The runoff generated for each grid cell is routed to the corner of each cell and a concentration time is determined before it enters into the river storage $\left(\mathrm{L}^{3} \mathrm{~L}^{-2} \mathrm{~T}^{-1}\right)$. See table S19. 44

$R_{G}^{1}$ runoff concentration in a grid cell of the first time step $\left(\mathrm{L}^{3} \mathrm{~L}^{-2} \mathrm{~T}^{-1}\right)$. See table S44. 69

$R_{h o}$ hortonian overland flow or infiltration excess overland flow occurs when precipitation exceeds the infiltration capacity of the soil. The water excess runs off over the ground surface because soil cannot absorb it $\left(\mathrm{L}^{3} \mathrm{~L}^{-2} \mathrm{~T}^{-1}\right)$. See tables $\mathrm{S} 20$, S21, S25. 45, 46, 50

$515 R_{\text {ho,calm }}$ hortonian overland flow in calm areas $\left(\mathrm{L}^{3} \mathrm{~L}^{-2} \mathrm{~T}^{-1}\right)$. See table $\mathrm{S} 21.46$

$R_{\text {ho,stormy }}$ hortonian overland flow in stormy areas $\left(\mathrm{L}^{3} \mathrm{~L}^{-2} \mathrm{~T}^{-1}\right)$. See table $\mathrm{S} 21.46$

$R_{i f}$ interflow or subsurface flow, outflow of the soil storage that discharges into river, lake, and wetland storages. It doesn't reach the groundwater storage $\left(\mathrm{L}^{3} \mathrm{~L}^{-2} \mathrm{~T}^{-1}\right)$. See tables $\mathrm{S} 14, \mathrm{~S} 17, \mathrm{~S} 20, \mathrm{~S} 26, \mathrm{~S} 43.39,42,45,51,68$

$R_{i f, f a s t}$ fast interflow from the unsaturated storage $\left(\mathrm{L}^{3} \mathrm{~L}^{-2} \mathrm{~T}^{-1}\right)$. This does not flow from the soil storage. See tables $\mathrm{S} 17$, S18, S26. 42, 43, 51

$R_{i f, \max }$ maximum subsurface flow $\left(\mathrm{L}^{3} \mathrm{~L}^{-2} \mathrm{~T}^{-1}\right)$. See table $\mathrm{S} 31.42$

$R_{\text {if,slow }}$ slow interflow from the unsaturated storage $\left(\mathrm{L}^{3} \mathrm{~L}^{-2} \mathrm{~T}^{-1}\right)$. This does not flow from the soil storage. See tables $\mathrm{S} 17$, S18, S26. 42, 43, 51

$R_{\text {in }}$ infiltration $\left(\mathrm{L}^{3} \mathrm{~L}^{-2} \mathrm{~T}^{-1}\right.$ ). See tables $\mathrm{S} 8, \mathrm{~S} 10, \mathrm{~S} 14, \mathrm{~S} 20, \mathrm{~S} 18, \mathrm{~S} 21, \mathrm{~S} 25, \mathrm{~S} 27, \mathrm{~S} 57.33,35,39,43,45,46,50,52,82$

$R_{i n, 0}$ corresponding point infiltration capacity $\left(\mathrm{L}^{3} \mathrm{~L}^{-2} \mathrm{~T}^{-1}\right)$. See tables S20, S24. 45, 49

$R_{i n, B L}$ infiltration from the base layer $(\mathrm{BL})\left(\mathrm{L}^{3} \mathrm{~L}^{-2} \mathrm{~T}^{-1}\right)$. See table $\mathrm{S} 30.55$

$R_{\text {in,cum }}$ cumulative infiltration $\left(\mathrm{L}^{3} \mathrm{~L}^{-2} \mathrm{~T}^{-1}\right)$. See table $\mathrm{S} 25.50$

$R_{i n, i}$ infiltration in layer $i\left(\mathrm{~L}^{3} \mathrm{~L}^{-2} \mathrm{~T}^{-1}\right)$. See tables $\mathrm{S} 8, \mathrm{~S} 14, \mathrm{~S} 20, \mathrm{~S} 25.33,39,45,50$

$R_{i n, i-1}$ infiltration in layer $i-1\left(\mathrm{~L}^{3} \mathrm{~L}^{-2} \mathrm{~T}^{-1}\right)$. See tables $\mathrm{S} 8, \mathrm{~S} 25.33,50$

$R_{i n, L}$ infiltration from layer $(\mathrm{L})\left(\mathrm{L}^{3} \mathrm{~L}^{-2} \mathrm{~T}^{-1}\right)$. See table $\mathrm{S} 26.51$ 
$R_{\text {in,max }}$ infiltration capacity or the maximum rate of infiltration $\left(\mathrm{L}^{3} \mathrm{~L}^{-2} \mathrm{~T}^{-1}\right)$. See tables $\mathrm{S} 20, \mathrm{~S} 21, \mathrm{~S} 24, \mathrm{~S} 25.45,46,49,50$

$R_{\text {in,over-so }}$ infiltration over soil covered cell fraction $\left(\mathrm{L}^{3} \mathrm{~L}^{-2} \mathrm{~T}^{-1}\right)$. See table $\mathrm{S} 25.50$

$R_{\text {in,pot }}$ potential infiltration $\left(\mathrm{L}^{3} \mathrm{~L}^{-2} \mathrm{~T}^{-1}\right)$. See table $\mathrm{S} 25.50$

$R_{i n, r}$ re-infiltration $\left(\mathrm{L}^{3} \mathrm{~L}^{-2} \mathrm{~T}^{-1}\right)$. See table $\mathrm{S} 14.39$

$535 R_{\text {in,sat }}$ infiltration at saturation level $\left(\mathrm{L}^{3} \mathrm{~L}^{-2} \mathrm{~T}^{-1}\right)$. See table S26. 51

$R_{i n, T L}$ infiltration from the top soil layer (TL) $\left(\mathrm{L}^{3} \mathrm{~L}^{-2} \mathrm{~T}^{-1}\right)$. See table $\mathrm{S} 20.45$

$R_{o f}$ runoff induced by the over saturation at the surface / is this similar to saturation excess flow $\left(\mathrm{L}^{3} \mathrm{~L}^{-2} \mathrm{~T}^{-1}\right)$. See table S20, S56. 45,81

$R_{p e}$ percolation or drainage, infiltrated water into the soil that runs off toward the groundwater storage $\left(\mathrm{L}^{3} \mathrm{~L}^{-2} \mathrm{~T}^{-1}\right)$. See tables S14, S18, S25, S26, S27, S29, S30, S39. 39, 50-52, 54, 55, 64

$R_{p e, h 2 o s f c}$ bottom drainage from the surface water store $\left(\mathrm{L}^{3} \mathrm{~L}^{-2} \mathrm{~T}^{-1}\right)$. See table $\mathrm{S} 25.50$

$R_{s}$ water that leaves the surface layer (topsoil layer) e.g. as overland flow / fast runoff in ISIMIP2b $\left(\mathrm{L}^{3} \mathrm{~L}^{-2} \mathrm{~T}^{-1}\right)$. See table S16. 40, 41

$R_{\text {sat }}$ saturation excess overland flow occurs when the soil is saturated or filled with water, and any additional precipitation or irrigation causes runoff. $\left(\mathrm{L}^{3} \mathrm{~L}^{-2} \mathrm{~T}^{-1}\right)$. See tables S20, S18, S22, S25. 43, 45, 47, 50

$R_{s b}$ sum of water that flows out from subsurface layer(s) including the groundwater layer (if present). Equals groundwater runoff in case of a groundwater layer below only one soil layer in ISIMIP2b $\left(\mathrm{L}^{3} \mathrm{~L}^{-2} \mathrm{~T}^{-1}\right)$. See table S17. 40, 42

$R_{s n}$ snow runoff, melted water that runs off on the ground surface covered with snow $\left(\mathrm{L}^{3} \mathrm{~L}^{-2} \mathrm{~T}^{-1}\right)$. See table S12. 37

$R_{\text {snwcp,ice }}$ ice runoff from snow-capped surfaces / is this a part of saturation excess flow $\left(\mathrm{M} \mathrm{L}^{-2} \mathrm{~T}^{-1}\right)$. See table S20. 45

$550 R_{\text {sof }, i}$ infiltration from soil layer $i\left(\mathrm{~L}^{3} \mathrm{~L}^{-2} \mathrm{~T}^{-1}\right)$. See table $\mathrm{S} 14.39$

$R_{\text {sof }, i-1}$ infiltration from soil layer $i-1\left(\mathrm{~L}^{3} \mathrm{~L}^{-2} \mathrm{~T}^{-1}\right)$. See table $\mathrm{S} 8.39$

$R_{s u}$ surface runoff or overland flow, water excess that runs off over the ground surface as Hortonian overland flow and (rainfall rate dependent) / or Saturation excess overland flow (soil saturated or filled with water) $\left(\mathrm{L}^{3} \mathrm{~L}^{-2} \mathrm{~T}^{-1}\right)$. See tables $\mathrm{S} 14$, S16, S18, S20, S22, S25, S32, S43, S44, S46. 39, 41, 43, 45, 47, 50, 57, 68, 69, 71

$R_{\text {su,ice }}$ ice water runoff at the land model resolution / is this the water that runs off over the ground surface covered with ice / part of saturation excess flow $\left(\mathrm{M} \mathrm{L}^{-2} \mathrm{~T}^{-1}\right)$. See table S20. 45

$R_{s u, l}$ liquid water runoff at the land model resolution / is this the saturation excess flow $\left(\mathrm{M} \mathrm{L}^{-2} \mathrm{~T}^{-1}\right)$. See tables S20, S43. 45,68

$R_{s u_{L C, R}}$ runoff generated for each cell that is routed towards the corner of each cell, with a concentration time, depending on land cover class, slope, and runoff component (surface, interflow, or baseflow). Runoff generated for a grid cell is then calculated using a triangular-weighting-function $\left(\mathrm{L}^{3} \mathrm{~L}^{-2} \mathrm{~T}^{-1}\right)$. See tables S19, S44. 44, 69

$R_{t o t}$ total runoff from land includes surface runoff, subsurface runoff, and groundwater recharge $\left(\mathrm{L}^{3} \mathrm{~L}^{-2} \mathrm{~T}^{-1}\right)$. See tables S14, S20, S18, S30, S35, S37, S43, S46. 39, 43, 45, 55, 60, 62, 68, 71

$R_{t o t, I S I M I P 2 b}$ total runoff ISIMIP2b, it includes surface runoff and subsurface runoff $\left(\mathrm{L}^{3} \mathrm{~L}^{-2} \mathrm{~T}^{-1}\right)$. See table S15. 40

$s_{r i}$ river bed slope $\left(\mathrm{L} \mathrm{L}^{-1}\right)$. See table $\mathrm{S} 46.71$ 
$s_{v p, s a t}$ slope of saturated vapour pressure $\left(\mathrm{M} \mathrm{L}^{-1} \mathrm{~T}^{-2} \Theta^{-1}\right)$. See table S2. 27

$s_{\text {we,mean }}$ mean slope within wetland (L). See table S42. 67

$S_{\text {buf }}$ storage buffer $\left(\mathrm{L}^{3} \mathrm{~L}^{-2} \mathrm{~T}^{-1}\right)$. See table S57. 82

$S_{c a}$ canopy compartment that retains water from precipitation and loses water through throughfall, stemflow and interception loss (evaporation) ( $\mathrm{L}^{3} \mathrm{~L}^{-2}$ ). See tables S3, S4, S5, S7, S20, S23, S26. 28-30, 32, 45, 48, 51

$S_{c a, \text { dif }}$ the difference between the canopy storage capacity and the water stored on the canopy ( $\left.\mathrm{L}^{3} \mathrm{~L}^{-2}\right)$. See table S5. 30

$S_{c a, \text { int }}$ canopy compartment that retains water after precipitation is intercepted by canopy $\left(\mathrm{L}^{3} \mathrm{~L}^{-2}\right)$. See table $\mathrm{S} 5.30$

$S_{c a, l}$ canopy compartment that retains rainfall and loses water through throughfall, stemflow and evaporation $\left(\mathrm{L}^{3} \mathrm{~L}^{-2}\right) . \mathrm{See}^{-}$ tables S3, S4, S5. 28-30

$S_{c a, \max }$ maximum value of canopy storage compartment $\left(\mathrm{L}^{3} \mathrm{~L}^{-2}\right)$. See tables $\mathrm{S} 4, \mathrm{~S} 5, \mathrm{~S} 7, \mathrm{~S} 20, \mathrm{~S} 23, \mathrm{~S} 49, \mathrm{~S} 51.29,30,32,45$, $48,74,76$

$S_{c a, \min }$ minimum value of canopy storage compartment $\left(\mathrm{L}^{3} \mathrm{~L}^{-2}\right)$. See table $\mathrm{S} 49.74$

$S_{c a, p}$ interception storage parameter (unitless). See tableS49. 74

$S_{c a, s}$ canopy compartment that retains snowfall and loses water through throughfall, stemflow and sublimation $\left(\mathrm{L}^{3} \mathrm{~L}^{-2}\right)$. See tables S3, S4, S5. 28-30

$S_{\text {cons }}$ conservative storage limit (unitless). See table S37. 62

$S_{f}$ storage of the fast response reservoir $\left(\mathrm{L}^{3} \mathrm{~T}^{-1}\right)$. See table $\mathrm{S} 22, \mathrm{~S} 26.47,51$

$S_{\text {flood }}$ flood storage limit (unitless). See table S37. 62

$S_{f t r}$ specified threshold of the fast response reservoir $\left(\mathrm{L}^{3} \mathrm{~T}^{-1}\right)$. See table S22. 47

$585 S_{g w}$ groundwater storage $\left(\mathrm{L}^{3} \mathrm{~L}^{-2}\right)$. See tables S29, S31, S53. 54, 56, 78

$S_{g w, n r w}$ groundwater storage non-renewable $\left(\mathrm{L}^{3} \mathrm{~L}^{-2}\right)$. See table S29. 54

$S_{g w, r w}$ groundwater storage renewable $\left(\mathrm{L}^{3} \mathrm{~L}^{-2}\right)$. See tables S29, S31, S53, S59, S69. 54, 56, 78, 84, 94

$S_{i}$ storage of $i$ element $=$ overland, baseflow, river or wetland (unitless). See table S52. 77

$S_{\text {ice }}$ ice storage $\left(\mathrm{L}^{3} \mathrm{~L}^{-2}\right)$. See table $\mathrm{S} 12.37$

$590 S_{i c e, s n, i+1}$ solid water stored in the upper snow layer $(i+1), i$ is the snow layer index $\left(\mathrm{L}^{3} \mathrm{~L}^{-2}\right) . \mathrm{See}$ table $\mathrm{S} 11.36$

$S_{l a}$ lake storage $\left(\mathrm{L}^{3} \mathrm{~L}^{-2}\right)$. See tables S32, S33, S34, S47. 57-59, 72

$S_{l a, \text { global }}$ global lake storage $\left(\mathrm{L}^{3} \mathrm{~L}^{-2}\right)$. See tables S32, S34. 57, 59

$S_{l a, g l o b a l, \max }$ maximum global lake storage $\left(\mathrm{L}^{3} \mathrm{~L}^{-2}\right)$. See tables S32, S49. 57, 76

$S_{\text {la,local }}$ local lake storage $\left(\mathrm{L}^{3} \mathrm{~L}^{-2}\right)$. See tables S32, S34. 57, 59

$S_{l a, l o c a l, \max }$ maximum local lake storage $\left(\mathrm{L}^{3} \mathrm{~L}^{-2}\right)$. See tables S32, S34, S49. 57, 59, 76

$S_{l a, \max }$ maximum amount of water in the lake storage $\left(\mathrm{L}^{3} \mathrm{~L}^{-2}\right)$. See table 334.59 
$S_{l, s n, i+1}$ liquid water stored in the upper snow layer $(i+1), i$ is snow layer index $\left(\mathrm{L}^{3} \mathrm{~L}^{-2}\right)$. See table $\mathrm{S} 11.36$

$S_{\text {norm }}$ normal storage limit (unitless). See table S37. 62

$S_{\text {paddy }}$ storage of flooded paddy rice $\left(\mathrm{L}^{3} \mathrm{~L}^{-2}\right)$. See table S22. 81

600

$S_{\text {pon }}$ ponding storage (sealed areas) $\left(\mathrm{L}^{3} \mathrm{~L}^{-2}\right)$. See table S20, S24. 45, 49

$S_{\text {pon, } \max }$ maximum ponding zone storage (L). See tables S20, S24. 45, 49

$S_{r e}$ reservoir storage $\left(\mathrm{L}^{3} \mathrm{~L}^{-2}\right)$. See tables S35, S37, S38. 60, 62, 63

$S_{\text {re, act }}$ actual reservoir storage $\left(\mathrm{L}^{3}\right)$. See table S35. 60

$S_{r e, C}$ reservoir storage capacity $\left(\mathrm{L}^{3}\right)$. See tables S35, S37, S49. 60, 62, 76

$605 S_{\text {re,global }}$ global reservoir storage $\left(\mathrm{L}^{3} \mathrm{~L}^{-2}\right)$. See tables S35, S43. 62, 68

$S_{r e, g l o b a l}^{\text {purpose }}$ global reservoir storage for irrigation or others purposes $\left(\mathrm{L}^{3} \mathrm{~L}^{-2}\right)$. See table S37. 62

$S_{\text {re }, \text { local }}$ local reservoir storage $\left(\mathrm{L}^{3} \mathrm{~L}^{-2}\right)$. See table S37. 62

$S_{r e, \max }$ maximum water amount of reservoir storage $\left(\mathrm{L}^{3} \mathrm{~L}^{-2}\right)$. See tables $\mathrm{S} 35, \mathrm{~S} 37.60,62$

$S_{r i}$ river storage $\left(\mathrm{L}^{3} \mathrm{~L}^{-2}\right)$. See tables $\mathrm{S} 43, \mathrm{~S} 45, \mathrm{~S} 46.68,70,71$

$610 S_{r i, n}$ state of the $n^{\text {th }}$ cascade in the river storage $\left(\mathrm{L}^{3} \mathrm{~L}^{-2}\right)$. See tables $\mathrm{S} 43, \mathrm{~S} 46, \mathrm{~S} 56.68,71,81$

$S_{r v}$ rivulet storage that collects water of small creeks, streams. $\left(\mathrm{L}^{3} \mathrm{~L}^{-2}\right)$. See tables $\mathrm{S} 19, \mathrm{~S} 44.44,69$

$S_{r z}$ root zone water storage $\left(\mathrm{L}^{3} \mathrm{~L}^{-2}\right)$. See tables $\mathrm{S} 7, \mathrm{~S} 18, \mathrm{~S} 24.32,43,49$

$S_{r z, \max }$ maximum root zone water storage or root zone storage capacity $\left(\mathrm{L}^{3} \mathrm{~L}^{-2}\right)$. See tables S7, S18, S24, S51. 32, 43, 49, 76

$615 S_{s n}$ snow storage, compartment that accumulates snow below freezing temperature and loses snow by melting and sublimation $\left(\mathrm{L}^{3} \mathrm{~L}^{-2}\right)$. See tables S8, S11, S12. 33, 36, 37

$S_{s o c}$ compartment that accumulates snow on canopy below freezing temperature and loses snow by melting and sublimation $\left(\mathrm{L}^{3} \mathrm{~L}^{-2}\right)$. See tables $\mathrm{S} 9, \mathrm{~S} 10.34,35$

$S_{s n, i}$ snow storage of layer $i\left(\mathrm{~L}^{3} \mathrm{~L}^{-2}\right)$. See table $\mathrm{S} 12.37$

$620 S_{s n, i}$ number of snow layers $i$ (unitless). See tables S8,S12. 33, 37

$S_{s n, i c e}$ frozen water content in snow storage $\left(\mathrm{L}^{3} \mathrm{~L}^{-2}\right)$. See table $\mathrm{S} 8.33$

$S_{s n, l}$ liquid water content in snow storage $\left(\mathrm{L}^{3} \mathrm{~L}^{-2}\right)$. See tables $\mathrm{S} 8, \mathrm{~S} 12.33,37$

$S_{s n, S G_{i}}$ subcompartment that accumulates snow in subgrid cells below freezing temperature and loses snow by melting and sublimation Müller Schmied et al. [34] $\left(\mathrm{L}^{3} \mathrm{~L}^{-2}\right)$. See table S12. 37

$S_{\text {suc }}$ compartment that accumulates snow under canopy below freezing temperature and loses snow by melting and sublimation $\left(\mathrm{L}^{3} \mathrm{~L}^{-2}\right)$. See table $\mathrm{S} 10.35$

$S_{s o}$ soil water storage compartment $\left(\mathrm{L}^{3} \mathrm{~L}^{-2}\right)$. See tables S14, S20, S18, S22, S23, S24, S27, S30, S49, S57. 27, 39, 43, 45, $47-49,55,76,82$ 
$S_{s o, 3 L}$ soil moisture or soil water content for the third layer (3L) $\left(\mathrm{L}^{3}\right)$. See table S27. 42

$630 S_{\text {so,crit }}$ critical volumetric soil moisture concentration that corresponds to a critical water suction potential $\left(\mathrm{L}^{3} \mathrm{~L}^{-2}\right) . \mathrm{See}^{-}$ tables S23, S24, S57. 48, 49, 82

$S_{s o, F_{i}}$ frozen soil water or frozen soil moisture at layer index $i\left(\mathrm{~L}^{3} \mathrm{~L}^{-2}\right)$. See tables $\mathrm{S} 14, \mathrm{~S} 29.39,54$

$S_{s o, F C}$ soil water content at field capacity (FC) $\left(\mathrm{L}^{3} \mathrm{~L}^{-2}\right)$. See tables S2, S20, S23, S24, S25, S30, S57. 27, 45, 48-50, 52, 55, 82

$635 S_{s o, F L}$ soil water content in first layer $(\mathrm{FL})\left(\mathrm{L}^{3} \mathrm{~L}^{-2}\right)$. See table S24. 49

$S_{s o, i}$ soil storage in layer $i\left(\mathrm{~L}^{3} \mathrm{~L}^{-2}\right)$. See tables $\mathrm{S} 14, \mathrm{~S} 24, \mathrm{~S} 25.39,49,50$

$S_{\text {so }, \text { ini }}$ initial soil water content or soil moisture $\left(\mathrm{L}^{3} \mathrm{~L}^{-2}\right)$. See table $\mathrm{S} 25.50$

$S_{s o, l, i}$ liquid soil storage in layer $i\left(\mathrm{~L}^{3} \mathrm{~L}^{-2}\right)$. See table $\mathrm{S} 14.39$

$S_{s o, \max }$ maximum soil storage $\left(\mathrm{L}^{3} \mathrm{~L}^{-2}\right)$. See tables S20, S23, S24, S30. 45, 48, 49, 55

$640 S_{s o, p o t}$ potential soil water content or soil moisture $\left(\mathrm{L}^{3} \mathrm{~L}^{-2}\right)$. See table $\mathrm{S} 25.50$

$S_{s o, \max , 3 L}$ maximum soil moisture or soil water content of the third soil layer (3L) (L). See tables S17, S30. 42, 55

$S_{\text {so,ready }}$ ready available soil water content $\left(\mathrm{L}^{3} \mathrm{~L}^{-2}\right)$. See table S57. 82

$S_{s o, r e l}$ relative soil water content or soil moisture $\left(\mathrm{L}^{3} \mathrm{~L}^{-2}\right)$. See tables $\mathrm{S} 21, \mathrm{~S} 23, \mathrm{~S} 24, \mathrm{~S} 25.46,48-50$

$S_{\text {so,SG }}$ subgrid soil storage $\left(\mathrm{L}^{3} \mathrm{~L}^{-2}\right)$. See table $\mathrm{S} 20.45$

$645 S_{s o, S G, \min }$ minimum subgrid soil moisture storage $\left(\mathrm{L}^{3} \mathrm{~L}^{-2}\right)$. See table $\mathrm{S} 20.45$

$S_{s o, S G, \max }$ maximum subgrid soil moisture storage $\left(\mathrm{L}^{3} \mathrm{~L}^{-2}\right)$. See table $\mathrm{S} 20.45$

$S_{\text {so,sat }}$ soil water content at saturation (SAT) $\left(\mathrm{L}^{3} \mathrm{~L}^{-2}\right)$. See tables S21, S25, S26, S28, S30, S57. 46, 50-53, 55, 77, 82

$S_{s o, s a t, i}$ soil water content at saturation in top layer $\left(\mathrm{L}^{3} \mathrm{~L}^{-2}\right)$. See table $\mathrm{S} 25.50$

$S_{s o, s a t, p}$ soil water content at saturation parameter (unitless). See tables S49, S50. 74, 75

$650 S_{s o, T 2 L}$ volumetric soil moisture content or soil storage for the top two layers (T2L) ( $\mathrm{L}^{3}$ ). See tables S20, S57. 45, 82

$S_{s o, T L}$ total available water capacity for the top soil layer $\left(\mathrm{L}^{3} \mathrm{~L}^{-1} \mathrm{~L}^{-2}\right)$. See tables $\mathrm{S} 18, \mathrm{~S} 22, \mathrm{~S} 49.43,47,75$

$S_{\text {so,cur }}$ current soil water content or current soil moisture $\left(\mathrm{L}^{3} \mathrm{~L}^{-2}\right)$. See table S52. 77

$S_{\text {so,tot }}$ total soil water content or total soil moisture $\left(\mathrm{L}^{3} \mathrm{~L}^{-2}\right)$. See tables S20, S21, S23, S24, S25, S26, S28, S30. 45, 46, $48-51,53,55$

$655 S_{s o, u F}$ unfrozen soil water or unfrozen soil moisture $\left(\mathrm{L}^{3} \mathrm{~L}^{-2}\right)$. See table S14. 38

$S_{s o, u F_{i}}$ unfrozen soil water or unfrozen soil moisture at layer index $i\left(\mathrm{~L}^{3} \mathrm{~L}^{-2}\right)$. See tables $\mathrm{S} 14, \mathrm{~S} 29.39,54$

$S_{s o, u F_{i, g w}}$ unfrozen soil water or unfrozen soil moisture at the layer $i$ that has groundwater table $\left(\mathrm{L}^{3} \mathrm{~L}^{-2}\right)$. See table S30. 55

$S_{s o, W P}$ soil water content at wilting point (WP) $\left(\mathrm{L}^{3} \mathrm{~L}^{-2}\right)$. See tables S20, S21, S23, S25, S26, S30, S52, S57. 45, 46, 48, 50, $51,55,77,82$ 
$S_{s o, F}$ frozen soil water or frozen soil moisture $\left(\mathrm{L}^{3} \mathrm{~L}^{-2}\right)$. See table $\mathrm{S} 14.38$

$S_{t o t}$ total reservoir storage capacity $\left(\mathrm{L}^{3}\right)$. See table S37. 62

$S_{u z}$ unsaturated zone storage $\left(\mathrm{L}^{3} \mathrm{~L}^{-2}\right)$, with two possible outflows: fast interflow and slow interflow. See tables S26, S30. 51, 55

$S_{u z, t h r}$ threshold for the unsaturated zone storage (L) which triggers fast interflow. See table S26. 51

$665 S_{w, f i r s t}$ the water storage at the beginning of the year $\left(\mathrm{L}^{3} \mathrm{~L}^{-2}\right)$. See table S37. 62

$S_{w e}$ wetland storage, compartment filled by precipitation or inflow and emptied by evapo(transpi)ration, outflow and groundwater recharge $\left(\mathrm{L}^{3} \mathrm{~L}^{-2}\right)$. See tables S39, S42, S56. 64, 67, 81

$S_{w e, g l o b a l}$ global wetland storage $\left(\mathrm{L}^{3} \mathrm{~L}^{-2}\right)$. See tables S39, S42. 64, 67

$S_{w e, g l o b a l, \text { max }}$ maximum global wetland storage $\left(\mathrm{L}^{3} \mathrm{~L}^{-2}\right)$. See tables S39, S49. 64, 76

$670 S_{\text {we }, \text { local }}$ local wetland storage $\left(\mathrm{L}^{3} \mathrm{~L}^{-2}\right)$. See tables S39, S42. 64, 67

$S_{\text {we,local,max }}$ maximum local wetland storage $\left(\mathrm{L}^{3} \mathrm{~L}^{-2}\right)$. See tables $\mathrm{S} 39, \mathrm{~S} 42$, S49. 64, 67, 76

$S_{y}$ specific yield depending on soil properties and water table location $\left(\mathrm{M} \mathrm{L}^{-1}\right)$. See table S29. 54

$S A I$ exposed stem area index (unitless). See tables S4, S6. 29, 31

$S R$ incoming solar radiation $\left(\mathrm{M} \mathrm{T}^{-3}\right)$. See table $\mathrm{S} 2.27$

$675 S W E$ snow water equivalent $\left(\mathrm{L}^{3} \mathrm{~L}^{-2} \mathrm{~T}^{-1}\right)$. See tables $\mathrm{S} 10, \mathrm{~S} 13.35,38$

$t$ time (T). See tables S3, S7, S8, S9, S10, S11, S13, S14, S19, S25, S29, S31, S32, S34, S35, S39, S43, S44, S46. 28, 32-36, $38,39,44,45,50,54,56,57,59,60,64,68,69,71$

$t_{r e t}$ topographic index of the retention time (L). See tables S43, S45, S46. 68, 70, 71

$t_{\text {ri,fast }}$ property of the fast reservoir $\left(25 \mathrm{~T} \mathrm{~L}^{-1}\right)$. See table $\mathrm{S} 43.68$

$680 t_{r i, s l o w}$ property of the slow reservoir $\left(3 \mathrm{~T} \mathrm{~L}^{-1}\right)$. See table $\mathrm{S} 43.68$

$t_{r i, s t r e a m}$ property of the stream reservoir $\left(0.24 \mathrm{~T} \mathrm{~L}^{-1}\right)$. See tables $\mathrm{S} 43, \mathrm{~S} 45, \mathrm{~S} 46.68,70,71$

$t_{w e}$ lag time for outflow computation of the wetland storage (T). See table S42. 67

$t_{d a y}$ day of year (L). See table S12. 37

$t_{\text {year }}$ number of days in the actual year (L). See tables S2, S12. 27, 37

$685 \operatorname{tri}_{x}$ triangular function (unitless). See table S19. 44

$T$ transpiration, water evaporated by plants through their stomata $\left(\mathrm{L}^{3} \mathrm{~L}^{-2} \mathrm{~T}^{-1}\right)$. See tables $\mathrm{S} 1, \mathrm{~S} 14, \mathrm{~S} 23, \mathrm{~S} 25, \mathrm{~S} 52.26,39$, $48,50,77$

$T_{a c t}$ actual transpiration, the initial water evaporated by plants through their stomata $\left(\mathrm{L}^{3} \mathrm{~L}^{-2} \mathrm{~T}^{-1}\right)$. See table S23. 48

$T_{c a}^{s n, c o v}$ transpiration of snow-covered canopy $\left(\mathrm{L}^{3} \mathrm{~L}^{-2} \mathrm{~T}^{-1}\right)$. See table $\mathrm{S} 23.48$

$690 T_{c a}^{\text {sn,free }}$ transpiration of snow-free canopy $\left(\mathrm{L}^{3} \mathrm{~L}^{-2} \mathrm{~T}^{-1}\right)$. See table $\mathrm{S} 23.48$ 
$T_{i}$ water removed by transpiration in each layer $i\left(\mathrm{~L}^{3} \mathrm{~L}^{-2} \mathrm{~T}^{-1}\right)$. See table $\mathrm{S} 14.39$

$T_{\text {max }}$ maximum transpiration $\left(\mathrm{L}^{3} \mathrm{~L}^{-2} \mathrm{~T}^{-1}\right)$. See table $\mathrm{S} 23.48$

$T_{\text {pot }}$ potential transpiration, water evaporated by plants through their stomata, if a sufficient water source is available $\left(\mathrm{L}^{3} \mathrm{~L}^{-2} \mathrm{~T}^{-1}\right)$. See table S23. 48

$U_{c a, s n}$ canopy snow unloading from wind speed and above-freezing temperatures $\left(\mathrm{L}^{3} \mathrm{~L}^{-2} \mathrm{~T}^{-1}\right)$. See table $\mathrm{S} 9.34$

$v$ flow velocity $\left(\mathrm{L} \mathrm{T}^{-1}\right)$. See tables $\mathrm{S} 45, \mathrm{~S} 46.70,71$

$v_{\text {mean }}$ mean flow velocity $\left(\mathrm{L} \mathrm{T}^{-1}\right)$. See table $\mathrm{S} 46.71$

$W$ wind speed $\left(\mathrm{L} \mathrm{T}^{-1}\right)$. See tables S2, S7, S11, S23, S24. 27, 32, 36, 48, 49

$W_{2}$ wind speed at $2 \mathrm{~m}$ height $\left(\mathrm{L} \mathrm{T}^{-1}\right)$. See tables S2, S23, S24. 27, 48, 49

$700 w_{s f c}$ balance of surface water (M). See table S25. 50

$X$ irrigation efficiency (unitless). See tables S54, S55, S57. 79, 80, 82

$X_{\text {conv }}$ conveyance efficiency (unitless). See table S57. 82

$X_{d o m}$ water use efficiency of the domestic sector (unitless). See tables S60, S63. 85, 88

$X_{e t}$ evaporation efficiency (unitless). See table S2. 27

$705 X_{\text {ind }}$ water use efficiency of the industrial sector (unitless). See tables S70, S73. 95, 98

$z$ gravitational potential $\left(\mathrm{M} \mathrm{L}^{-1} \mathrm{~T}^{-2}\right)$. See table $\mathrm{S} 13.38$

$Z$ vertical coordinate (L). See table S28. 53

$Z_{c r}$ matric head induced by capillary action (L). See table S28. 53

$\alpha$ empirical parameter (unitless). See tables S13, S24, S25, S46, S52. 38, 49, 50, 71, 77

$710 \beta$ empirical shape parameter. It needs to be fitted during the calibration processes (unitless). See tables S13, S18, S21, S24, S25, S31, S46. 38, 43, 46, 49, 50, 56, 71

$\gamma$ psychrometric constant. See tables S2, S7, S11, S23, S24. 27, 32, 36, 48, 49

$\Lambda_{r z}$ root zone soil moisture stress parameter (unitless). See table S7. 32

$\Gamma$ gamma function (unitless). See tables S31, S46. 56, 71

$715 \delta$ time variation (unitless). See tables S3, S8, S9, S10, S11, S12, S13, S14, S20, S24, S25, S28, S29, S30, S32, S35, S39, S43, S44, S45, S46. 28, 33-35, 37-39, 45, 49, 50, 53-55, 57, 60, 64, 68-71

$\Delta t$ time step (T). See tables S4, S5, S7, S8, S9, S10, S11, S12, S13, S20, S25, S30, S34, S35, S37, S43, S45, S46, S53, S59, S69. 29, 30, 32-38, 45, 50, 52, 55, 59, 62, 70, 71, 77, 78, 84, 94

$\epsilon_{c}$ empirical constant (17.8), found by calibration [25] (unitless). See table S2. 27

720

$\zeta_{s n}$ snow layer thickness of layer $j\left(\mathrm{~L}^{3} \mathrm{~L}^{-2}\right)$. See table $\mathrm{S} 12.37$

$\zeta_{s o, i}$ soil layer thickness of layer $i(\mathrm{~L})$. See table S13, S52. 38, 77 
$\eta$ parameter (unitless). See table S31. 56

$\theta$ air temperature $(\Theta)$. Note: in the equations, air temperature is in Kelvin degrees. See tables S2, S7, S8, S9, S10, S11, S12, S25. 27, 32-35, 37, 50

$\theta_{\text {day, } \max }$ daily maximum air temperature $(\Theta)$. See table $\mathrm{S} 2.27$

$\theta_{\text {day,mean }}$ daily mean air temperature $(\Theta)$. See table $\mathbf{S} 2.27$

$\theta_{\text {day,min }}$ daily minimum air temperature $(\Theta)$. See table S2. 27

$\theta_{f}$ triple point temperature for water $\Theta$ ). See table $\mathrm{S} 12.37$

$\theta_{\text {freeze }}$ freezing temperature $(\Theta)$. See table S12.37

$\theta_{\min }$ minimum air temperature $(\Theta)$. See table S7. 32

$\theta$, month mean monthly air temperature $(\Theta)$. See table S2. 27

$\theta_{M}$ air temperature above $0(\Theta)$. See tables $\mathrm{S} 8, \mathrm{~S} 12.33,37$

$\theta_{s n}$ snow temperature $(\Theta)$. See table S12. 37

$\theta_{s n, i}$ snow temperature of layer $i(\Theta)$. See table S12. 37

$\theta_{\text {so }}$ soil temperature $(\Theta)$. See tables S12, S23, S25. 37, 48, 50

$\theta_{s o, i}$ soil temperature of soil layer $i(\Theta)$. See table S13. 38

$\theta_{s o, i+1}$ soil temperature of soil layer $i+1(\Theta)$. See table S13. 38

$\theta_{s u}$ temperature of the surface layer $(\Theta)$. See table S10. 35

$\theta_{S G_{i}}$ mean air temperature in a subgrid cell Müller Schmied et al. [34] ( $\Theta$ ). See tables S8, S12, S13. 33, 37, 38

$\theta_{\text {veg }}$ vegetation temperature $(\Theta)$. See table S23. 48

$\iota$ storage parameter, defined as the hydraulic retention time of a single linear reservoir segment of length $l_{r i}$. It can be calculated as the average travel time of water through a single river segment (unitless). See table S46. 71

$\kappa$ calibration constant $\left(2.3 \times 10^{3}\right)$ that approximately compensates for the differences in advection or in vapour transfer effect [25] (unitless). See tables S2. 27

$745 \kappa_{g w}$ retention time for water in the groundwater storage (L). See table S30. 56

$\kappa_{o l}$ retention time for water in the overland flow storage (L). See table S44. 44

$\kappa_{r i}$ retention time for water in the river flow storage (L). See tables S43, S46. 68, 71

$\lambda$ water distribution uniformity scalar, depending on the irrigation system. See table S57. 82

$\nu$ decay coefficient $(\mathrm{T})$. See table S13. 38

$750 \Pi_{R_{\text {in }}}$ infiltration shape parameter (unitless). See tables S20, S24. 45, 49

$\rho_{\text {air }}$ density of atmospheric air $\left(\mathrm{M} \mathrm{L}^{-3}\right)$. See tables S2, S7, S11, S23, S24. 27, 32, 36, 48, 49

$\rho_{\text {ice }}$ intrinsic density of ice $\left(\mathrm{M} \mathrm{L}^{-3}\right)$. See table $\mathrm{S} 12.37$ 
$\rho_{\text {sn }, i}$ snow density of layer $i\left(\mathrm{M} \mathrm{L}^{-3}\right)$. See table $\mathrm{S} 12.37$

$\rho_{w}$ density water $\left(\mathrm{M} \mathrm{L}^{-3}\right)$. See tables $\mathrm{S} 8, \mathrm{~S} 9, \mathrm{~S} 10, \mathrm{~S} 11.33,35,36,77$

$\sigma$ subgrid topographical variability (unitless). See table S20. 45

$\tau$ time constant (T). See table S25. 50

$v$ scale parameter (unitless). See tables S31, S24. 49, 56

$\phi_{a, i}$ simulated actual soil moisture content of layer $i\left(\mathrm{~L}^{3} \mathrm{~L}^{-3}\right)$. See tables S52, S56. 77, 81

$\phi_{\text {min }, i}$ minimum soil moisture content of layer $i$ that results in no water stress in that layer $\left(\mathrm{L}^{3} \mathrm{~L}^{-3}\right)$. See table S56. 81

$\phi_{r, i}$ residual soil moisture content of layer $i\left(\mathrm{~L}^{3} \mathrm{~L}^{-3}\right)$ See tables $\mathrm{S} 24, \mathrm{~S} 25.49,50$

$\phi_{s, i}$ maximum soil moisture content of layer $i\left(\mathrm{~L}^{3} \mathrm{~L}^{-3}\right)$ See table $\mathrm{S} 25.50,75,81$

$\phi_{\text {so }}$ soil porosity $\left(\mathrm{L}^{3}\right)$. See tables $\mathrm{S} 13, \mathrm{~S} 20, \mathrm{~S} 22.38,45,77$

$\phi_{t, i}$ target soil moisture content of layer $i\left(\mathrm{~L}^{3} \mathrm{~L}^{-3}\right)$. See tables S52, S56. 77, 81

$\phi_{w, i}$ soil moisture limit above which the actual transpiration is equated with the PET at layer $i\left(\mathrm{~L}^{3} \mathrm{~L}^{-3}\right)$. See table S24. 49

$765 \chi$ constant, energy needed to melt ice $\left(\mathrm{M} \mathrm{L}^{-2} \mathrm{~T}^{-2} \mathrm{~L}^{-3}\right)$. See table S12. 37

$\chi_{f}$ energy of fusion $\left(\mathrm{M} \mathrm{L}^{2} \mathrm{~T}^{-3} \mathrm{~L}^{-2}\right)$. See table S12. 37

$\chi_{M}$ energy flux given to the pack because of liquid water refreezing or removed from the pack during melt $\left(\mathrm{M} \mathrm{L}^{-2} \mathrm{~T}^{-2} \mathrm{~L}^{-3}\right)$. See tables S8, S10. 33, 35

$\chi_{r s}$ energy flux advected to the snowpack by rain or snow $\left(\mathrm{M} \mathrm{L}^{-2} \mathrm{~T}^{-2} \mathrm{~L}^{-3}\right)$. See tables $\mathrm{S} 10, \mathrm{~S} 12.35,37$

$770 \chi_{\text {to }}$ total energy $\left(\mathrm{M} \mathrm{L}^{2} \mathrm{~T}^{-2} \mathrm{~L}^{-2}\right)$. See table $\mathrm{S} 12.37$

$\Psi$ matric potential( $\left.\mathrm{M} \mathrm{L}^{-1} \mathrm{~T}^{-2}\right)$. See table S13. 38

$\Psi_{i}$ soil matric potential of layer $i\left(\mathrm{M} \mathrm{L}^{-1} \mathrm{~T}^{-2}\right)$. See table S28. 53

$\Psi_{j w t}$ matric potential of the layer directly above the water table $\left(\mathrm{M} \mathrm{L}^{-1} \mathrm{~T}^{-2}\right)$. See table S30. 55

$\Psi_{\text {sat }}$ saturated soil matric potential $\left(\mathrm{M} \mathrm{L}^{-1} \mathrm{~T}^{-2}\right)$. See table S13. 38

$\Psi_{\text {sat }, i}$ saturated soil matric potential of layer $i\left(\mathrm{M} \mathrm{L}^{-1} \mathrm{~T}^{-2}\right)$. See table S28. 53

$\Psi_{w t}$ matric potential at the water table (i.e. 0) $\left(\mathrm{M} \mathrm{L}^{-1} \mathrm{~T}^{-2}\right)$. See table S30. 55 
Table S1. Actual evapotranspiration $A E T$

\begin{tabular}{ll}
\hline Model & Equation \\
\hline CLM4.5 & $A E T=E_{c a}+E_{s o}+T$ \\
CLM5.0 & $A E T=E_{c a}+E_{s o}+T$ \\
CWatM & $A E T=E_{c a}+E_{l a}+E_{r e}+E_{r i}+E_{s n}+E_{s o}+T$ \\
DBH & $A E T=E_{c a}+E_{s o}+T$ \\
H08 & $A E T=E_{s o}$ \\
JULES-W1 & $A E T=E_{c a}+E_{s n}+E_{s o}+T$ \\
LPJmL & $A E T=E_{c a}+E_{l a}+E_{r e}+E_{r i}+E_{s n}+E_{s o}+T$ \\
Mac-PDM.20 & $A E T=E_{c a}+E_{s o}$ \\
MATSIRO & $A E T=E_{c a}^{s n, c o v}+E_{c a}^{s n, f r e e}+E_{s n}+E_{s o, i c e}+E_{s o, l}^{s n, f r e e g r}$ \\
mHM & $A E T=E_{c a}+E T_{s o, i}$ \\
MPI-HM & $A E T=A_{i r r}+E_{s o}+E_{w e}+T$ \\
ORCHIDEE & $A E T=E_{c a}+E_{f l o o d p l a i n}+E_{s n}+E_{s o}$ \\
PCR-GLOBWB & $A E T=E_{c a}+E_{l a}+E_{r e}+E_{r i}+E_{s o}$ \\
VIC & $A E T=\sum_{n=1}^{N} f_{c a, n} \times\left(E_{c a}+T\right)+f_{s o, b a r e} \times E_{s o}$ \\
& for: $\sum_{n=1}^{N+1} f_{c a, n}=1$ see details [28]; [29]; [20] \\
& $A E T=E_{c a}+E_{l a}+E_{r e}+E_{s n}+E_{s o}+E_{w e}$ \\
WaterGAP2 & $A E T=E_{s o}$ \\
WAYS &
\end{tabular}


Table S2. Potential evapotranspiration PET

\begin{tabular}{|c|c|}
\hline Model & Equation \\
\hline CLM4.5 & not represented \\
\hline CLM5.0 & not represented \\
\hline CWatM & $P E T=\frac{0.408 \times s_{v p, s a t} \times\left(N R_{n}-H_{s o}\right)+\gamma \times \frac{900}{\theta} \times W_{2} \times\left(e_{s a t}+e_{a c t}\right)}{s_{v p, s a t}+\gamma \times\left(1+0.34 \times W_{2}\right)}$ \\
\hline DBH & not represented \\
\hline H08 & $\begin{array}{l}P E T= \begin{cases}\rho_{a i r} \times r_{w} \times\left(q_{s a t}^{g r}-q_{g r}\right), & \text { snow present } \\
\rho_{\text {air }} \times r_{w} \times\left(q_{s a t}^{g r}-q_{g r}\right) \times \frac{\left(1+\text { zeta } \times X_{e t}\right)}{(1+z e t a)}, & \text { snow absence }\end{cases} \\
\text { Where zeta is } \zeta \text { in Milly }[31] . \\
\text { And } X_{\text {et }}= \begin{cases}1.0, & 0.75 \times S_{\text {so }, F C}<S_{\text {so }} \\
\frac{W \times S_{s o}}{0.75 \times S_{s o, F C}}, & 0.75 \times S_{s o, F C}>S_{\text {so }}\end{cases} \end{array}$ \\
\hline JULES-W1 & $P E T=\frac{0.408 \times s_{v p, s a t} \times\left(N R_{n}-H_{s o}\right)+\gamma \times \frac{900}{\theta} \times W_{2} \times\left(e_{s a t}+e_{a c t}\right)}{s_{v p, s a t}+\gamma \times\left(1+0.34 \times W_{2}\right)}$ \\
\hline LPJmL & $P E T=\frac{s_{v p, s a t}}{\left(s_{v p, s a t}+\gamma \times \theta\right)} \times \frac{2 \times P A \kappa}{H_{l, E}}$ \\
\hline Mac-PDM.20 & $P E T=\frac{s_{v p, s a t} \times\left(N R_{n}-H_{s o}\right)+\gamma \times c_{a i r} \times\left(e_{s a t}-e_{s a t, d e w}\right) \times r_{w}}{(597.3-(\theta-273.15) \times 0.564) \times\left(s_{v p, s a t}+\gamma \times\left(1+\frac{r_{w}}{r_{c a}}\right)\right)}$ \\
\hline MATSIRO & $\begin{array}{l}P E T=\frac{0.408 \times s_{v p, s a t} \times\left(N R_{n}-H_{s o}\right)+\gamma \times \frac{900}{\theta} \times W_{2} \times\left(e_{s a t}+e_{a c t}\right)}{s_{v p, s a t}+\gamma \times\left(1+0.34 \times W_{2}\right)} \\
\text { (used only in crop growth scheme) }\end{array}$ \\
\hline $\begin{array}{l}\text { mHM } \\
\text { MPI-HM }\end{array}$ & $\begin{array}{l}\left.P E T=\kappa \times S R \times\left(\left(\theta_{\text {day, mean }}-273.15\right)+\epsilon_{c}\right) \times \sqrt{\theta_{\text {day,max }}-\theta_{\text {day }, \text { min }}}\right) . \text { See [25] } \\
P E T=\frac{0.408 \times s_{v p, \text { sat }} \times\left(N R_{n}-H_{s o}\right)+\gamma \times \frac{900}{\theta} \times W_{2} \times\left(e_{\text {sat }}+e_{\text {act }}\right)}{s_{v p, \text { sat }}+\gamma \times\left(1+0.34 \times W_{2}\right)}\end{array}$ \\
\hline $\begin{array}{l}\text { ORCHIDEE } \\
\text { PCR-GLOBWB }\end{array}$ & $\begin{array}{l}P E T=\rho_{\text {air }} \times C_{\text {bulk }} \times W \times\left(q_{\text {sat }}^{\text {gr }}-q\right) . \text { See }[33] ;[31] \\
P E T=1 \times 0.165 \times 216.7 \times t_{\text {year }} \times \frac{e_{\text {sat }}}{\left(\theta_{, \text {month }}-273.15\right)+273.3}\end{array}$ \\
\hline VIC & $P E T=\frac{0.408 \times s_{v p, s a t} \times\left(N R_{n}-H_{s o}\right)+\rho_{a i r} \times c_{a i r} \times \frac{\left(e_{s a t}+e_{a c t}\right)}{r_{w}}}{s_{v p, s a t}+\gamma}$ \\
\hline WaterGAP2 & $\begin{array}{l}\text { PET }=C_{P T} \times \frac{s_{v p, \text { sat }}}{s_{v p, s a t}+\gamma} \times N R \\
C_{P T}= \begin{cases}1.26, \quad \mathrm{G}=\text { humid } \\
1.74, \quad \mathrm{G}=\text { arid or semi-arid }\end{cases} \\
s_{v p, s a t}=\frac{4098 \times 0.6108 \times \exp \left(\frac{17.27 \times(\theta-273.15)}{\theta-35.85}\right)}{(\theta-35.85)^{2}} \\
\gamma=\frac{0.0016286 \times 101.3}{H_{l}} \quad \begin{array}{ll}\left.2.501+0.334(\mathrm{MJ} \mathrm{kg})^{-1}\right), \\
2.501-0.002361\left(\mathrm{MJ} \mathrm{kg}^{-1}\right) \times(\theta-273.15), & \theta \geq 273.15\end{array} . \text { See [13] }\end{array}$ \\
\hline WAYS & $P E T=\frac{0.408 \times s_{v p, s a t} \times\left(N R_{n}-H_{s o}\right)+\gamma \times \frac{900}{\theta} \times W_{2} \times\left(e_{s a t}+e_{a c t}\right)}{s_{v p, s a t}+\gamma \times\left(1+0.34 \times W_{2}\right)}$ \\
\hline
\end{tabular}


Table S3. Canopy storage $\left(S_{c a}\right)$

\begin{tabular}{|c|c|c|c|}
\hline \multirow[t]{2}{*}{ Model } & \multirow[t]{2}{*}{ Equation } & \multicolumn{2}{|l|}{ Water Flux } \\
\hline & & Inflows & Outflows \\
\hline CLM4.5 & $\frac{\delta S_{c a}}{\delta t}=P_{i n t}-\left(P_{t h, l}+P_{t h, s}\right)-E_{c a}$ & $P_{\text {int }}$ & $\begin{array}{l}P_{t h, l} P_{t h, s} \\
E_{c a}\end{array}$ \\
\hline CLM5.0 & $\frac{\delta S_{c a}}{\delta t}=P_{i n t}-\left(P_{t h, l}+P_{t h, s}\right)-E_{c a}$ & $P_{\text {int }}$ & $\begin{array}{l}P_{t h, l} P_{t h, s} \\
E_{c a}\end{array}$ \\
\hline CWatM & $\frac{\delta S_{c a}}{\delta t}=P_{t o t}-P_{t h}-E_{c a}$ & $P_{t o t}$ & $E_{c a} P_{t h}$ \\
\hline DBH & $\frac{\delta S_{c a}}{\delta t}=P_{t o t}-P_{t h}-E_{c a}$ & $P_{t o t}$ & $E_{c a} P_{t h}$ \\
\hline H08 & not represented & & \\
\hline JULES-W1 & $\frac{\delta S_{c a}}{\delta t}=P_{t o t}-P_{t h}-E_{c a}$ & $P_{t o t}$ & $E_{c a} P_{t h}$ \\
\hline LPJmL & $\frac{\delta S_{c a}}{\delta t}=P_{t o t}-P_{t h}-E_{c a}$ & $P_{t o t}$ & $E_{c a} P_{t h}$ \\
\hline Mac-PDM.20 & not represented (all water intercepted is assumed to evaporate) & & \\
\hline MATSIRO & $\begin{array}{l}\frac{\delta S_{c a}}{\delta t}=\frac{\delta S_{c a, l}}{\delta t}+\frac{\delta S_{c a, s}}{\delta t} \\
\frac{\delta S_{c a, l}}{\delta t}=P_{i n t, l}-P_{t h, l}-E_{c a, l}\end{array}$ & $P_{i n t, l} P_{i n t, s}$ & $\begin{array}{ll}E_{c a, l} & E_{c a, s} \\
P_{t h, l} & P_{t h, s}\end{array}$ \\
\hline $\mathrm{mHM}$ & $\begin{array}{l}\frac{\delta S_{c a, s}}{\delta t}=P_{i n t, s}-P_{t h, s}-E_{c a, s} \\
\frac{\delta S_{c a}}{\delta t}=P_{t o t}-P_{t h}-E_{c a}\end{array}$ & $P_{t o t}$ & $E_{c a} P_{t h}$ \\
\hline MPI-HM & not represented & & \\
\hline ORCHIDEE & $\frac{\delta S_{c a}}{\delta t}=P_{t o t}-P_{t h}-E_{c a}$ & $P_{t o t}$ & $E_{c a} P_{t h}$ \\
\hline PCR-GLOBWB & $\frac{\delta S_{c a}}{\delta t}=P_{t o t}-P_{t h}-E_{c a}$ & $P_{t o t}$ & $E_{c a} P_{t h}$ \\
\hline VIC & $\frac{\delta S_{c a}}{\delta t}=P_{t o t}-P_{t h}-E_{c a}$ See $[28]$ & $P_{t o t}$ & $E_{c a} P_{t h}$ \\
\hline WaterGAP2 & $\frac{\delta S_{c a}}{\delta t}=P_{t o t}-P_{t h}-E_{c a}$ & $P_{t o t}$ & $E_{c a} P_{t h}$ \\
\hline WAYS & $\frac{\delta S_{c a}}{\delta t}=P_{t o t}-P_{t h}-E_{c a}$ & $P_{t o t}$ & $E_{c a} P_{t h}$ \\
\hline
\end{tabular}


Table S4. Precipitation intercepted by canopy storage $\left(P_{\text {int }}\right)$

\begin{tabular}{|c|c|}
\hline Model & Equation \\
\hline CLM4.5 & $\begin{array}{l}P_{i n t}=0.25 \times\left(P_{r a}+P_{s n}\right) \times(1-\exp (-0.5 \times(L A I+S A I))) \\
0.25=\text { scales interception from point to grid cell }\end{array}$ \\
\hline CLM5.0 & $P_{i n t}=0.25 \times\left(P_{r a}+P_{s n}\right) \times(1-\exp (-0.5 \times(L A I+S A I)))$ \\
\hline CWatM & $\begin{array}{l}0.25=\text { scales interception from point to grid cell } \\
P_{\text {int }}=\min \left(P_{t o t}, S_{c a, \max }\right) \\
S_{c a, \max } \text { from Global Land Cover Characteristics database version } 2.0 \text { varying every } 10 \text { days depending } \\
\text { on land use class }\end{array}$ \\
\hline $\begin{array}{l}\text { DBH } \\
\text { H08 } \\
\text { JULES-W1 } \\
\text { LPJmL } \\
\text { Mac-PDM.20 }\end{array}$ & $\begin{array}{l}P_{\text {int }}=P_{\text {tot }} \times\left(f_{c a}+f_{c a} \times \exp \frac{C_{P, l} \times L A I}{f_{c a}}\right) \\
\text { not represented } \\
\text { not represented } \\
\text { not represented } \\
P_{\text {int }}=I_{\text {cap }} \times\left(1.0-\exp \left(-0.5 \times P_{\text {tot }}\right)\right)\end{array}$ \\
\hline MATSIRO & $\begin{array}{l}P_{\text {int }, l}=\left(B_{\text {stormy }} \times P_{\text {int }, l, \text { stormy }}\right)+\left(\left(1-B_{\text {stormy }}\right) \times P_{\text {int }, l, \text { calm }}\right) \\
P_{\text {int }, s}=\left(B_{\text {stormy }} \times P_{\text {int }, s, \text { stormy }}\right)+\left(\left(1-B_{\text {stormy }}\right) \times P_{\text {int }, s, \text { calm }}\right)\end{array}$ \\
\hline & $\begin{array}{l}P_{\text {int }, l, \text { stormy }}=\min \left(f_{L A I} \times\left(P_{g r, l, c a l m}+\frac{P_{g r, l, s t o r m y}}{B_{\text {stormy }}}\right), \frac{S_{c a, \text { max }}-S_{c a, l}}{\Delta t}\right) \\
P_{\text {int }, l, \text { calm }}=\min \left(f_{L A I} \times P_{g r, l, c a l m}, \frac{S_{c a, \text { max }}-S_{c a, l}}{\Delta t}\right)\end{array}$ \\
\hline & $P_{i n t, s, s t o r m y}=\min \left(f_{L A I} \times\left(P_{g r, s, c a l m}+\frac{P_{g r, s, s t o r m y}}{B_{\text {stormy }}}\right), \frac{S_{c a, m a x}-S_{c a, s}}{\Delta t}\right)$ \\
\hline & $P_{i n t, s, c a l m}=\min \left(f_{L A I} \times P_{g r, s, c a l m}, \frac{S_{c a, \max }-S_{c a, s}}{\Delta t}\right)$ \\
\hline $\mathrm{mHM}$ & $P_{i n t}=\min \left(P_{t o t}, S_{c a, \max }\right)$ \\
\hline MPI-HM & not represented \\
\hline ORCHIDEE & not represented \\
\hline PCR-GLOBWB & not represented \\
\hline VIC & not represented \\
\hline WaterGAP2 & $P_{i n t}=P_{t o t}-\left(S_{c a, \max }-S_{c a}\right)$ \\
\hline WAYS & $P_{i n t, l}=P_{r a}-\left(S_{c a, \max }-S_{c a}\right)$ \\
\hline
\end{tabular}


Table S5. Throughfall $\left(P_{t h}\right)$

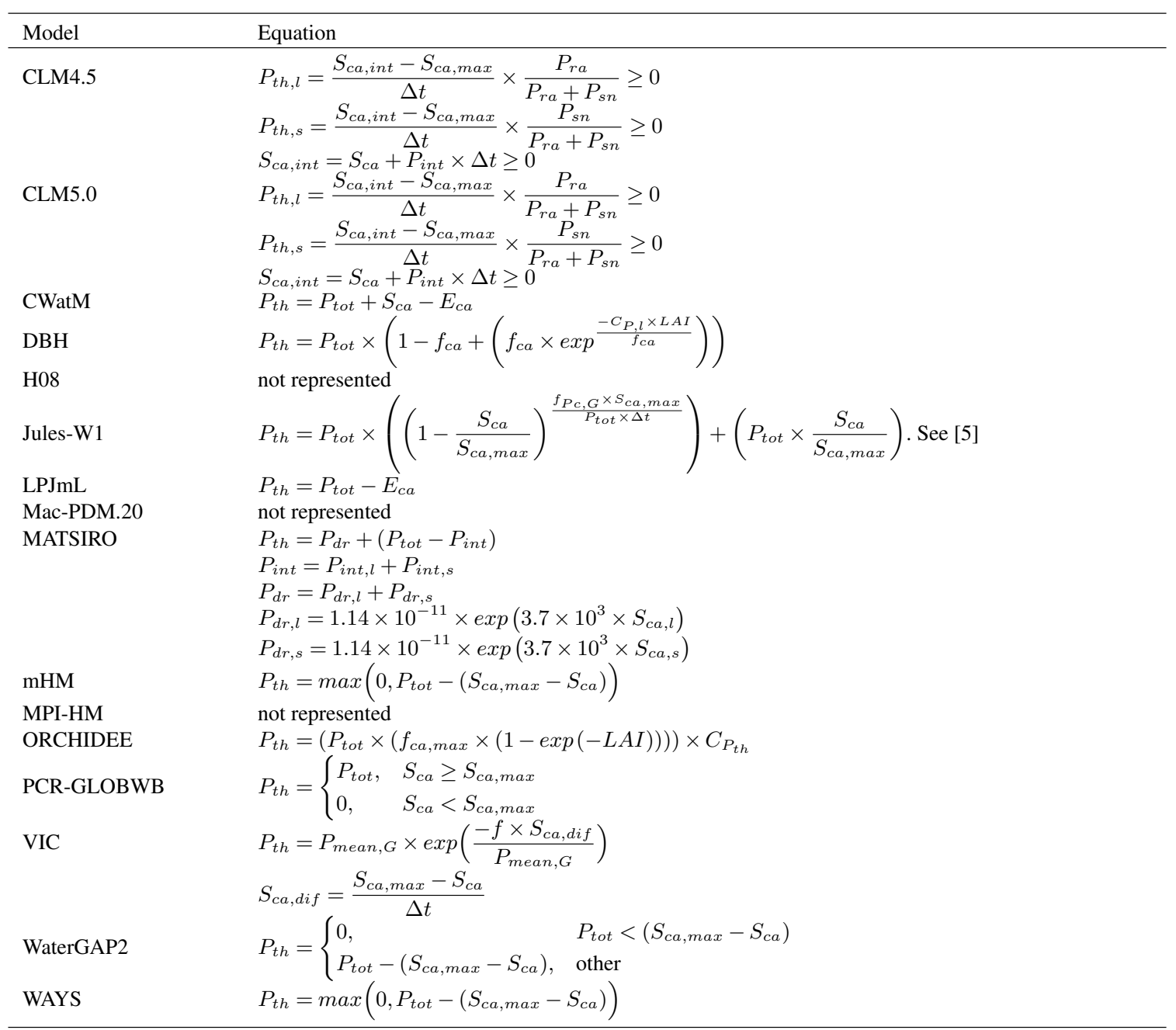


Table S6. Precipitation falls directly to the ground $\left(P_{g r}\right)$

\begin{tabular}{ll}
\hline Model & Equation \\
\hline CLM4.5 & $P_{g r, l}=P_{r a} \times(1-0.25 \times(1-\exp (-0.5 \times(L A I+S A I))))$ \\
& $P_{g r, s}=P_{s n} \times(1-0.25 \times(1-\exp (-0.5 \times(L A I+S A I))))$ \\
CLM5.0 & $P_{g r, l}=P_{r a} \times(1-0.25 \times(1-\exp (-0.5 \times(L A I+S A I))))$ \\
& $P_{g r, s}=P_{s n} \times(1-0.25 \times(1-\exp (-0.5 \times(L A I+S A I))))$ \\
CWatM & not represented \\
DBH & not represented \\
H08 & no canopy compartment, rainfall and snowfall fall directly to the ground. $P_{g r}=P_{r a}+P_{s n}$ \\
JULES-W1 & not represented \\
LPJmL & not represented \\
Mac-PDM.20 & not represented \\
MATSIRO & computed same as table S5 \\
mHM & not represented \\
MPI-HM & not represented \\
ORCHIDEE & not represented \\
PCR-GLOBWB & not represented \\
VIC & $P_{g r}=-\left(\frac{P_{\text {mean }, G}}{f_{B_{P}}}\right) \times \ln \left(f_{P}\right) ;$ for $0<f_{P} \leq 1$. See details [29] \\
WaterGAP2 & not represented \\
WAYS & not represented \\
\hline
\end{tabular}


Table S7. Canopy evaporation $\left(E_{c a}\right)$

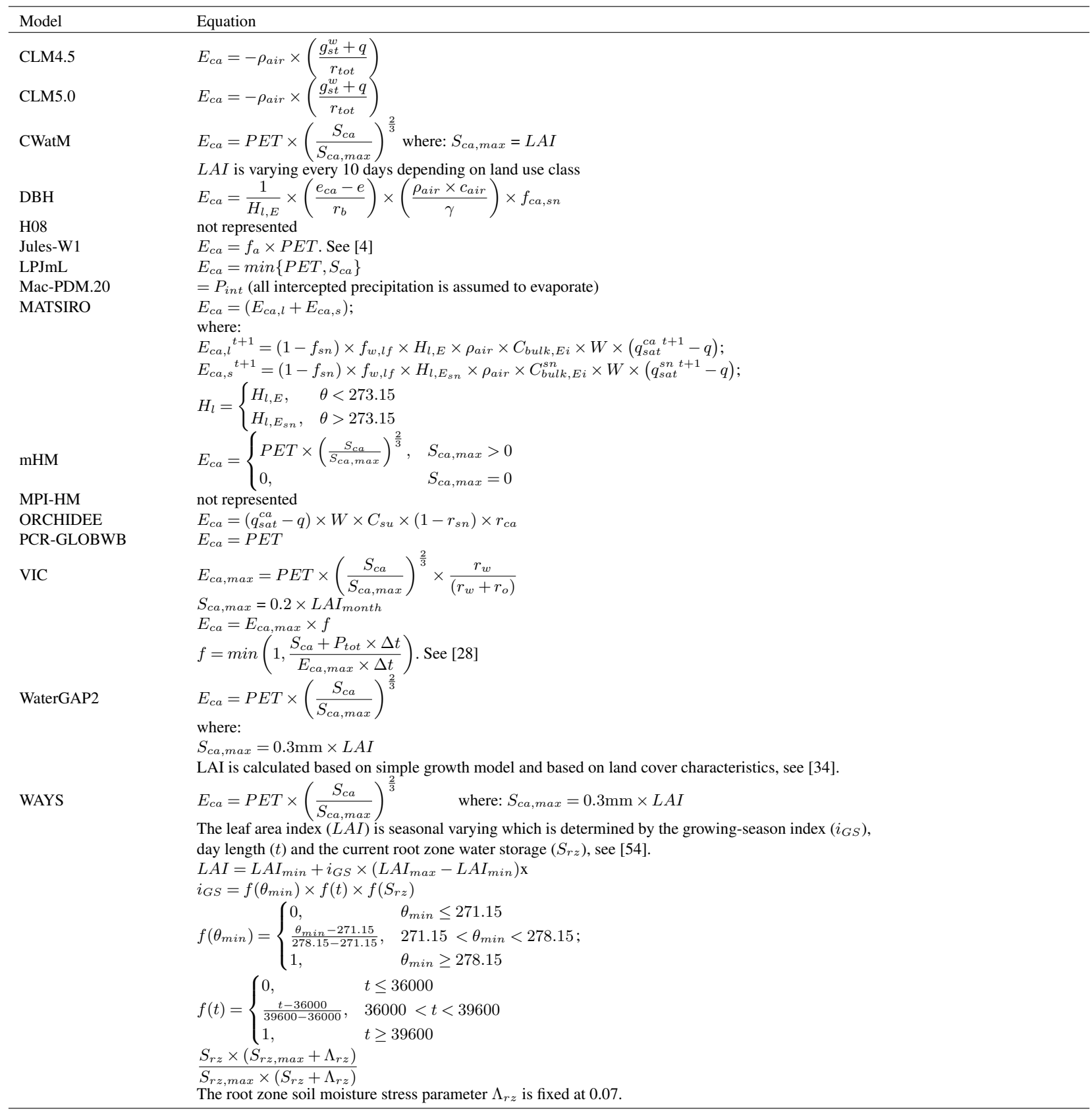


Table S8. Snow storage $S_{s n}$

\begin{tabular}{|c|c|c|c|}
\hline \multirow[t]{2}{*}{ Model } & \multirow[t]{2}{*}{ Equation } & \multicolumn{2}{|l|}{ Water Flux } \\
\hline & & Inflows & Outflows \\
\hline CLM4.5 & $\begin{array}{l}\frac{\delta S_{s n, i c e}}{\delta t}= \begin{cases}f_{s n} \times\left(P_{s n}+\left(F-E_{s n}\right)\right)-M, & i=S_{s n, i}+1 \\
-M, & i=S_{s n, i}+2, \ldots, 0\end{cases} \\
\frac{\delta S_{s n, l}}{\delta t}= \begin{cases}\left(f_{s n} \times\left(P_{\text {ra }}+\left(E_{\text {dew }}-E_{\text {so }}\right)\right)-M_{\text {out }}\right)+M, & i=S_{\text {sn }, i}+1 \\
\left(M_{\text {in }}-M_{\text {out }}\right)+M, & i=S_{\text {sn }, i}+2, \ldots, 0\end{cases} \end{array}$ & $\begin{array}{l}P_{s n} P_{r a} E_{d e w} \\
F\end{array}$ & $E_{\text {sn }} M M_{\text {out }}$ \\
\hline CLM5.0 & $\begin{array}{l}\frac{\delta S_{s n, i c e}}{\delta t}= \begin{cases}f_{s n} \times\left(P_{s n}+\left(F-E_{s n}\right)\right)-M, & i=S_{s n, i}+1 \\
-M, & i=S_{s n, i}+2, \ldots, 0\end{cases} \\
\frac{\delta S_{s n, l}}{\delta t}= \begin{cases}\left(f_{s n} \times\left(P_{r a}+\left(E_{\text {dew }}-E_{\text {so }}\right)\right)-M_{\text {out }}\right)+M, & i=S_{s n, i}+1 \\
\left(M_{\text {in }}-M_{\text {out }}\right)+M, & i=S_{\text {sn }, i}+2, \ldots, 0\end{cases} \end{array}$ & $\begin{array}{l}P_{s n} P_{r a} E_{d e w} \\
F\end{array}$ & $E_{\text {sn }} M M_{\text {out }}$ \\
\hline CWatM & $\begin{array}{l}\frac{\delta S_{s n}}{\delta t}=P_{s n}-M-E_{s n} \\
P_{s n}= \begin{cases}P_{t o t}, & \theta<273.15 \\
0, & \theta>273.15\end{cases} \end{array}$ & $P_{s n}$ & $M E_{s n}$ \\
\hline DBH & $\frac{\delta S_{s n}}{\delta t}=P_{t o t}-E_{s n}-R_{i n}$ & $P_{t o t}$ & $E_{s n} R_{i n}$ \\
\hline H08 & $\frac{\delta S_{s n}}{\delta t}=P_{s n}-M-E_{s n}$ & $P_{s n}$ & $M E_{s n}$ \\
\hline JULES-W1 & $\frac{\partial S_{s n}}{\delta t}=P_{t o t}-M-E_{s n}$ & $\begin{array}{l}P_{t o t} \text { for } \\
\theta<273.15\end{array}$ & $M E_{s n}$ \\
\hline LPJmL & $\frac{\delta S_{s n}}{\delta t}= \begin{cases}S_{s n}-P_{t h}-M-E_{s n}, & \theta<273.15 \\
S_{s n}-M-E_{s n}, & \theta>273.15\end{cases}$ & $P_{t h}$ & $M E_{s n}$ \\
\hline Mac-PDM.20 & $\frac{\delta S_{s n}}{\delta t}=P_{s n}-M P_{s n}= \begin{cases}P_{t o t}, & \theta<\theta_{M} \\
0, & \theta \geq \theta_{M}\end{cases}$ & $P_{s n}$ & $M$ \\
\hline MATSIRO & $\frac{\delta S_{s n}}{\delta t}=P_{s, c a}-E_{s n}-M+F$ & $P_{s, c a} F$ & $E_{s n} M$ \\
\hline $\mathrm{mHM}$ & $\frac{\delta S_{s n}}{\delta t}=P_{s n}-M P_{s n}= \begin{cases}P_{t o t}, & \theta<\theta_{M} \\
0, & \theta \geq \theta_{M}\end{cases}$ & $P_{s n}$ & $M$ \\
\hline MPI-HM & $\begin{array}{l}\frac{\delta S_{s n}}{\delta t}=\frac{\delta S_{s n, i c e}}{\delta t}+\frac{\delta S_{s n, l}}{\delta t} \\
\frac{\delta S_{s n, i c e}}{\delta t}=P_{s n}-M+F \\
\frac{\delta S_{s n, l}}{\delta t}=M_{p o t}-M-F\end{array}$ & $P_{s n} F$ & $M$ \\
\hline ORCHIDEE & $\begin{array}{l}F= \begin{cases}S_{s n, l}, & \theta<273.15 \\
0, & \theta \geq 273.15\end{cases} \\
\frac{\delta S_{s n, i}}{\delta t}= \begin{cases}P_{s n}+P_{r a}-R_{i n, i}-M_{, i}-E_{s n}, & i=1 \\
R_{i n, i-1}-R_{i n, i}-M_{i}, & i=2,3\end{cases} \\
\text { See [53] }\end{array}$ & $P_{s n} P_{r a}$ & $R_{i n, i} M E_{s n}$ \\
\hline PCR-GLOBWB & $\frac{\delta S_{s n}}{\delta t}=P_{s n}-M$ & $P_{s n}$ & $M$ \\
\hline VIC & $\begin{array}{l}\frac{\delta S_{s n, l}}{\delta t}=P_{r a}+\left(\frac{H_{l}}{\rho_{w} \times H_{l, E}}-\frac{\chi_{M}}{\rho_{w} \times H_{M}}\right) \times \Delta t \\
\frac{\delta S_{s n, i c e}}{\delta t}=P_{s n}+\left(\frac{H_{l}}{\rho_{w} \times H_{l, E}}+\frac{\chi_{M}}{\rho_{w} \times H_{M}}\right) \times \Delta t . \text { See [2] }\end{array}$ & $P_{r a} P_{s n}$ & $\begin{array}{l}H_{l} H_{l, E} \\
\chi_{M} H_{M}\end{array}$ \\
\hline WaterGAP2 & $\begin{aligned} \frac{\delta S_{s n}}{\delta t} & =\frac{1}{100} \times \sum_{i=1}^{100}\left(P_{s, S G_{i}}-M_{S G_{i}}-E_{s n, S G_{i}}\right) \\
P_{s, S G_{i}} & = \begin{cases}P_{t o t}, & \theta_{S G_{i}}<273.15 \\
0, & \text { other }\end{cases} \end{aligned}$ & $P_{s n}$ & $M_{s} E_{s n}$ \\
\hline WAYS & $\frac{\delta S_{s n}}{\delta t}=P_{s n}-M$ & $P_{s n}$ & $M$ \\
\hline
\end{tabular}


Table S9. Snow held on the canopy $S_{s o c}$

\begin{tabular}{|c|c|c|c|}
\hline \multirow[t]{2}{*}{ Model } & \multirow[t]{2}{*}{ Equation } & \multicolumn{2}{|l|}{ Water Flux } \\
\hline & & Inflows & Outflows \\
\hline CLM4.5 & Interception by vegetation does not distinguish between liquid and solid phases & & \\
\hline CLM5.0 & $\frac{\delta S_{s o c}}{\delta t}=P_{i n t, s}-\left(P_{d r, s}-U_{c a, s n}\right) \times \Delta t-E_{l f s m} \times \Delta t$ & $P_{\text {int }, s}$ & $\begin{array}{l}E_{l f s m} U_{c a, s n} \\
P_{d r, s}\end{array}$ \\
\hline CWatM & not represented & & \\
\hline $\mathrm{DBH}$ & $\begin{array}{l}\frac{\delta S_{s o c}}{\delta t}=P_{t o t}-P_{t h}-P_{d r}-E_{c a} \\
\text { for ambient temperature lower than the freezing temperature, precipitation is retreated as snow. }\end{array}$ & $P_{t o t}$ & $P_{t h} P_{d r} E_{c a}$ \\
\hline H08 & not represented & & \\
\hline JULES-W1 & $\frac{\delta S_{s o c}}{\delta t}=\left(0.7 \times\left((4.4 \times L A I)-I_{0}\right) \times\left(1-\exp ^{\frac{-P_{t o t}}{4.4 \times L A I}}\right)\right)-E_{s n}-M_{c a}-0.4 \times M_{c a}$. See [5] & $\begin{array}{l}P_{t o t} \text { for } \\
\theta<273.15 ; I_{0}\end{array}$ & $M_{c a} E_{s n}$ \\
\hline $\begin{array}{l}\text { LPJmL } \\
\text { Mac-PDM.20 }\end{array}$ & $\begin{array}{l}\text { not represented } \\
\text { not represented }\end{array}$ & & \\
\hline MATSIRO & $\frac{\delta S_{s o c}}{\delta t}=P_{i n t, s}-P_{d r, s}-E_{c a, s}$ & $P_{\text {int }, s}$ & $P_{d r, s} E_{c a, s}$ \\
\hline mHM & not represented & & \\
\hline MPI-HM & not represented & & \\
\hline ORCHIDEE & not represented & & \\
\hline PCR-GLOBWB & not represented & & \\
\hline VIC & $\begin{array}{l}\frac{\delta S_{s o c}}{\delta t}=f_{L A I} \times P_{s n} \\
f_{L A I}=0.6 \text { See }[2] .[48]\end{array}$ & $P_{s n}$ & \\
\hline WaterGAP2 & not represented & & \\
\hline WAYS & not represented & & \\
\hline
\end{tabular}


Table S10. Snow under canopy $S_{\text {suc }}$

\begin{tabular}{|c|c|c|c|}
\hline \multirow[t]{2}{*}{ Model } & \multirow[t]{2}{*}{ Equation } & \multicolumn{2}{|l|}{ Water Flux } \\
\hline & & Inflows & Outflows \\
\hline CLM4.5 & see table $\mathrm{S} 8$ & & \\
\hline $\begin{array}{l}\text { CLM5.0 } \\
\text { CWatM }\end{array}$ & $\begin{array}{l}\frac{\delta S_{s u c}}{\delta t}=f_{s n} \times\left(P_{\text {int }, l}+E_{\text {dew }}-E_{c a, l}\right) \times \Delta t \\
\text { not represented }\end{array}$ & $P_{\text {int }, l} E_{\text {dew }}$ & $E_{c a, l}$ \\
\hline $\mathrm{DBH}$ & $\frac{\delta S_{s u c}}{\delta t}=P_{g r, s}-E_{s n, s o}-R_{i n}$ & $P_{g r, s}$ & $E_{s n, s o} R_{i n}$ \\
\hline H08 & not represented & & \\
\hline JULES-W1 & $\frac{\delta S_{s u c}}{\delta t}=P_{t o t}-\frac{S_{s o c}}{\delta t}-M_{\text {underca }}-E_{\text {snunderca }}$ & $\begin{array}{l}P_{t o t} \text { for } \\
\theta<273.15\end{array}$ & $\begin{array}{l}M_{\text {underca }} \\
E_{\text {snunderca }}\end{array}$ \\
\hline LPJmL & not represented & & \\
\hline Mac-PDM.20 & not represented & & \\
\hline MATSIRO & see table $\mathrm{S} 8$ & & \\
\hline $\mathrm{mHM}$ & not represented & & \\
\hline MPI-HM & not represented & & \\
\hline ORCHIDEE & see table $\mathrm{S} 8$ & & \\
\hline PCR-GLOBWB & not represented & & \\
\hline VIC & $\rho_{w} \times c_{i c e} \times \frac{\delta S_{s u c} \times \theta_{s u}}{\Delta t}=H_{l}+H_{s e}+N R+\chi_{M}+\chi_{r s}$. See [2] & $P_{t o t}$ & $S W E$ \\
\hline $\begin{array}{l}\text { WaterGAP2 } \\
\text { WAYS }\end{array}$ & $\begin{array}{l}\text { not represented } \Delta t \\
\text { not represented }\end{array}$ & & \\
\hline
\end{tabular}


Table S11. Sublimation $E_{s n}$

\begin{tabular}{|c|c|}
\hline Model & Equation \\
\hline CLM4.5 & $E_{s n}=E_{s o}-\max \left(E_{s o} \times \frac{S_{l, s n, i+1}}{S_{i c e, s n, i+1}+S_{l, s n, i+1}}, 0\right)$ for $E_{s o} \geq 0$ \\
\hline CLM5.0 & $E_{s n}=\min \left(A E T, \frac{S_{s n}}{\Delta t}\right)$ \\
\hline CWatM & $E_{s n}=\min \left(M, E_{s o}\right)$ \\
\hline DBH & $E_{s n}=\frac{1}{H_{l, E}} \times\left(\frac{e_{c a}-e}{r_{b}}\right) \times\left(\frac{\rho_{a i r} \times c_{a i r}}{\gamma}\right) \times f_{c a, s n} \times \frac{H_{l, E}}{H_{l, E}+H_{l, E_{s n}}}$ \\
\hline H08 & $E_{s n}=P E T$ \\
\hline JULES-W1 & $\begin{array}{l}E_{s n}=\frac{\rho_{a i r}}{r_{s o}+r_{c a}} \times\left(q_{\text {sat }}^{c a}-q\right) \\
E_{\text {snunderca }}=\frac{\rho_{a i r}}{f_{a h}+r_{c a}} \times\left(q_{s a t}^{g r}-q\right) . \text { See [17]; [47] } \\
E_{s n}= \begin{cases}0.1\left(\mathrm{~mm} \mathrm{day}^{-1}\right), & S_{s n} \geq 0.1(\mathrm{~mm}) \\
0\left(\mathrm{~mm} \mathrm{day}^{-1}\right), & S_{s n}<0.1(\mathrm{~mm})\end{cases} \end{array}$ \\
\hline Mac-PDM.20 & not represented \\
\hline MATSIRO & $\begin{array}{l}E_{\text {sn }}^{\text {freegr }}{ }^{t+1}=\left(1-f_{s n}\right) \times f_{F} \times H_{l, E_{s n}} \times \rho_{\text {air }} \times C_{\text {bulk }, E g} \times W \times\left(q_{s u} \times q_{\text {sat }}^{\text {gr }}{ }^{t+1}-q\right) \\
E_{\text {sn }}^{\text {covgrt }+1}=f_{\text {sn }} \times H_{l, E_{s n}} \times \rho_{\text {air }} \times C_{\text {bulk, }}^{\text {sn }} \times W \times\left(q_{\text {sat }}^{\text {gr, snt }}+1\right. \\
-q)\end{array}$ \\
\hline mHM & not represented \\
\hline MPI-HM & not represented \\
\hline $\begin{array}{l}\text { ORCHIDEE } \\
\text { PCR-GLOBWB }\end{array}$ & $\begin{array}{l}E_{s n}=\left(q_{s a t}^{c a}-q\right) \times W \times C_{s u} \times\left(1-r_{\text {floodplain }}\right) \times r_{s n} \\
\text { not represented }\end{array}$ \\
\hline VIC & $E_{s n}=\frac{H_{l, E}}{\rho_{w} \times H_{l, E_{s n}}}$. See [2] \\
\hline $\begin{array}{l}\text { WaterGAP2 } \\
\text { WAYS }\end{array}$ & $\begin{array}{l}E_{s n, S G_{i}}=P E T \text { see }[42] \\
\text { not represented }\end{array}$ \\
\hline
\end{tabular}


Table S12. Snowmelt $M$

\begin{tabular}{|c|c|}
\hline Model & Equation \\
\hline CLM4.5 & $M=\frac{H_{i} \times \Delta t}{H_{M}}$ for $i=S_{s n, i}+1, \ldots, 0$ \\
\hline CLM5.0 & $M=\min \left(S_{i c e}, \frac{\chi}{H_{M}}\right)$ \\
\hline CWatM & $\begin{array}{l}M=C_{m} \times C_{m, \text { season }} \times\left(1+0.01 \times P_{r a}\right) \times\left(\theta_{S G_{i}}-\theta_{M}\right) \times \Delta t-E_{s n} ; \text { See }[45] \\
\text { for } 10 \text { elevation zones per grid. } \\
M_{\text {glacier }}=C_{m \text { glacier }} \times C_{m, \text { seasonglacier }} \times\left(\theta_{S G_{i}}-\theta_{M}\right) \\
\text { for } 3 \text { elevation zones per grid }\end{array}$ \\
\hline DBH & $M=\theta_{\text {freeze }} \times\left(H_{\text {tot }}+\delta H_{\text {tot }}\right)-\left(\theta_{\text {so }} \times H_{\text {tot }}+\theta \times \delta H_{\text {tot }}\right)$ \\
\hline H08 & $\begin{aligned} M= & \frac{\chi_{f}}{H_{M, i c e}} \text { with } H_{M, i c e}=0.333 \times 10^{6} \\
& \min \left(S_{s n}, \frac{\theta-\theta_{M}}{H_{M} \times H_{-}}\right)\end{aligned}$ \\
\hline JULES-W1 & 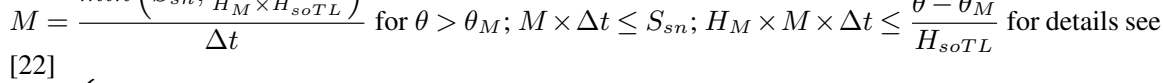 \\
\hline LPJmL & $M= \begin{cases}\frac{H}{0.3 \chi^{9}}, & \theta>273.15 \\
R_{s n}=P_{t h}, & S_{s n}>20.000(\mathrm{~mm})\end{cases}$ \\
\hline Mac-PDM.20 & $M= \begin{cases}\min \left(S_{s n}, C_{m} \times(\theta-273.15)\right), & \theta>273.15 \\
0, & \theta \leq 273.15\end{cases}$ \\
\hline MATSIRO & $\begin{array}{l}M=\sum_{i=1} M_{t} \\
\text { for } k=1, N \\
\text { for } k=1(\text { starting from top layer }) \\
\text { for } S_{s n(1)} \neq 0 \text { and } \theta_{M}<\theta_{s n 1}\end{array}$ \\
\hline & $\begin{array}{l}H_{s n}=H_{s n 2}-H_{s n 1}-\frac{c_{i c e} \times S_{s n 1} \times\left(\theta_{M}-\theta_{s n 1}\right)}{\Delta t} \\
M=\min \left(\frac{S_{s n 1}}{\Delta t}, \frac{H_{s n}}{H_{M}}\right)\end{array}$ \\
\hline & $\begin{array}{l}M_{1}=f_{s n} \times \bar{M} \\
H_{s n}=H_{s n}-\left(M \times H_{M}\right) \\
\text { for } 2 \leq k \leq N\end{array}$ \\
\hline & $\begin{array}{l}\theta_{s n, k}=\theta_{s n, k}+\frac{H_{s n}}{c_{i c e} \times S_{s n, k}} \times \Delta t \\
H_{s n}=\max \left(\frac{c_{i c e} \times S_{s n, k} \times\left(\theta_{s n, k}-\theta_{M}\right)}{\Delta t}, 0\right)\end{array}$ \\
\hline & $M=\min \left(\frac{S_{s n, i}}{\Delta t}, \frac{H_{s n}}{H_{M}}\right)$ \\
\hline $\mathrm{mHM}$ & $\begin{array}{l}M_{i}=f_{s n} \times M \\
M=\min \left(S_{s n},\left(\theta-\theta_{M}\right) \times C_{m}\right) \quad \text { where } \theta>\theta_{M}\end{array}$ \\
\hline MPI-HM & $M_{\text {pot }}=\max \left(0,\left(\sin \left(\pi \times \frac{t_{\text {day }}}{t_{\text {year }}}\right) \times 8.3+0.7\right) \times\left(\theta-\theta_{\text {freeze }}\right)\right)$ \\
\hline ORCHIDEE & $\begin{array}{l}M=M_{\text {pot }}+\max \left(0, S_{s n, l}-0.06 \times S_{s n}\right) \\
M_{i}=S_{s n, i}-\frac{1.9 \times 10^{6} \times \frac{\rho_{s n, i}}{\rho_{i c e}} \times \zeta_{s n} \times\left(\theta_{s n, i}-\theta_{f}\right)-H_{s n, i}}{H_{M, i c e}} . \text { See [53] }\end{array}$ \\
\hline $\begin{array}{l}\text { PCR-GLOBWB } \\
\text { VIC }\end{array}$ & $\begin{array}{l}M=\min \left(S_{s n},\left(\theta-\theta_{M}\right) \times C_{m}\right) \quad \text { where } \theta>\theta_{M} \\
\chi_{t o}=\left(N R+H_{s e}+H_{l}+\chi_{r s}\right) \times \Delta t . \text { See [2] }\end{array}$ \\
\hline WaterGAP2 & $\begin{aligned} M & = \begin{cases}C_{m} \times\left(\theta_{S G_{i}}-273.15\right), & \theta_{S G_{i}}>273.15, S_{s n, S G_{i}}>0 \mathrm{~mm} \\
0, & \text { other }\end{cases} \\
C_{m} & =\text { landcover specific degree-day factor, see [34] }\end{aligned}$ \\
\hline WAYS & $M= \begin{cases}\min \left(S_{s n}, C_{m} \times(\theta-273.15)\right), & \theta>273.15 \\
0, & \theta \leq 273.15\end{cases}$ \\
\hline
\end{tabular}


Table S13. Frozen soil

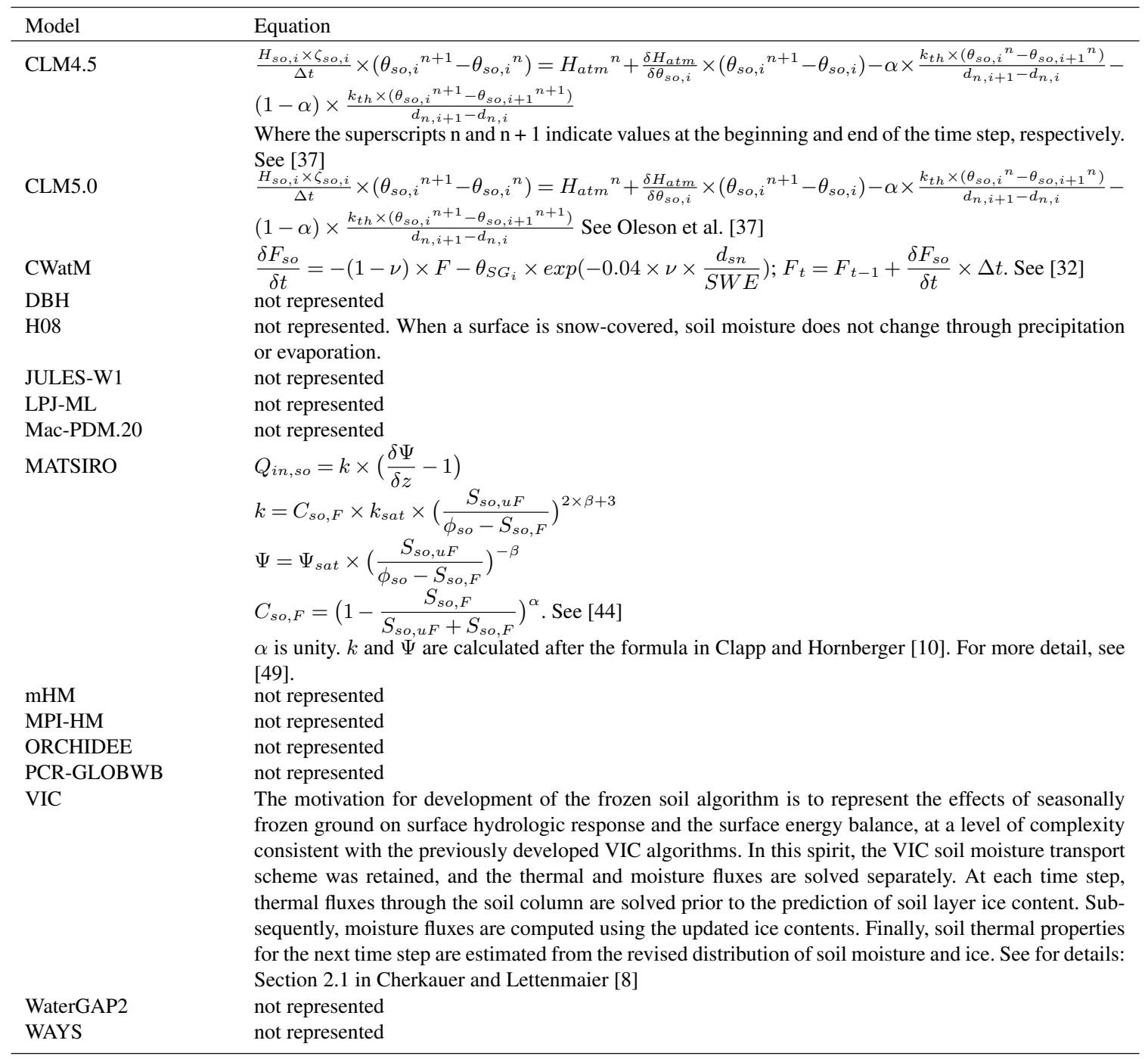


Table S14. Soil storage $S_{\text {so }}$

\begin{tabular}{|c|c|c|c|}
\hline \multirow[t]{2}{*}{ Model } & \multirow[t]{2}{*}{ Equation } & \multicolumn{2}{|l|}{ Water Flux } \\
\hline & & Inflows & Outflows \\
\hline CLM4.5 & $\frac{\delta d_{s o} \delta S_{s o, l, i}}{\delta t}=Q_{i}-Q_{i-1}-T_{i}$ & $Q_{i}$ & $Q_{i-1} T_{i}$ \\
\hline CLM5.0 & $\frac{\delta d_{s o} \delta S_{s o, l, i}}{\delta t}=Q_{i}-Q_{i-1}-T_{i}$ & $Q_{i}$ & $Q_{i-1} T_{i}$ \\
\hline CWatM & $\frac{\delta S_{s o}}{\delta t}=R_{c r}+R_{i n}-R_{g w r}-R_{i f}-E_{s o}-T$ & $R_{c r} R_{i n}$ & $\begin{array}{l}E_{s o} R_{g w r} R_{i f} \\
T\end{array}$ \\
\hline $\mathrm{DBH}$ & $\frac{\delta S_{s o, i}}{\delta t}= \begin{cases}R_{i n, i}-E_{s o, i}-R_{g w r, i}, & \mathrm{i}=0 \text { (top layer) } \\
R_{g w r, i-1}-R_{g w r, i}-T_{i}, & i>0\end{cases}$ & $R_{i n, i} R_{g w r, i-1}$ & $E_{s o, i} R_{g w r, i} T_{i}$ \\
\hline $\mathrm{H} 08$ & $\frac{\delta S_{s o, i}}{\delta t}=M+P_{t h}-E_{s o}-R_{i n}$ & $M P_{t h}$ & $E_{\text {so }} R_{\text {in }}$ \\
\hline JULES-W1 & $\frac{\delta S_{s o}}{\delta t}=M+P_{t h}-E_{s o}-R_{i f}-R_{s u}$ & $M P_{t h}$ & $E_{s o} R_{i f} R_{s u}$ \\
\hline LPJmL & $\frac{\delta S_{s o}}{\delta t}=\left(R_{i f}+R_{i n}\right)-R_{g w r}-R_{i f}-E_{s o}-T$ & $R_{i f} R_{i n}$ & $E_{\text {so }} R_{\text {gwr }} T$ \\
\hline Mac-PDM.20M & $\frac{O N_{s o}}{\delta t}=M+P_{t o t}-E_{s o}-R_{s u}$ & $P_{\text {tot }} M$ & $E_{s o} R_{s u}$ \\
\hline MATSIRO & $\frac{\partial S_{s o}}{\delta t}=\sum_{n=1,13}\left[\left(S_{s o, F_{i}}+S_{s o, u F_{i}}\right) \times d_{s o}\right]$ & & \\
\hline $\mathrm{mHM}$ & $\frac{\delta S_{s o, i}}{\delta t}= \begin{cases}R_{\text {in }, i}-E T_{\text {so }, i}-R_{\text {sof }, i}, & \mathrm{i}=0 \text { (top soil layer) } \\
R_{\text {sof }, i-1}-E T_{\text {so }, i}-R_{\text {sof }, i}, & i>0\end{cases}$ & $R_{i n, i}$ & $E T_{s o, i} R_{s o f, i}$ \\
\hline MPI-HM & $\frac{\delta S_{s o}}{\delta t}=R_{i n}-R_{p e}-E_{s o}-T$ & $R_{\text {in }}$ & $E_{s o} R_{p e} T$ \\
\hline ORCHIDEE & $\frac{\delta S_{s o}}{\delta t}=R_{i n}+R_{i n, r}-R_{s u}-E_{s o}$ & $R_{i n} R_{i n, r}$ & $E_{s o} R_{s u}$ \\
\hline PCR-GLOBWB & $\frac{\partial S_{s o}}{\delta t}=R_{i n}-R_{g w r}-R_{i f}-E_{s o}-T$ & $R_{i n}$ & $\begin{array}{l}E_{s o} R_{g w r} R_{i f} \\
T\end{array}$ \\
\hline VIC & $\begin{array}{l}\frac{\delta S_{s o}}{\delta t}=\frac{\delta}{\delta d_{s o}} \times\left(D_{w} \times \frac{\delta S_{s o}}{\delta d_{s o}}\right)+\frac{\delta k}{\delta d_{s o}} \\
\text { for the top two soil layers: }\end{array}$ & $\begin{array}{l}D_{w d_{s o, 1}} k_{d_{s o, 1}} \\
R_{\text {in }}\end{array}$ & $\begin{array}{ll}k_{d_{s o, 1}} & D_{w d_{s o, 2}} \\
E_{s o} T & R_{g w}\end{array}$ \\
\hline & $\begin{array}{l}\frac{\delta S_{s o}}{\delta t} \times d_{s o, i}=R_{i n}-E_{s o}-T-k \times d_{s o, i}-D_{w} \times d_{s o, i} \\
\text { for details see Gao et al. [20]; Liang et al. [29] } \\
\text { for lower soil layer: } \\
\frac{\delta S_{s o 3}}{\delta t} \times\left(d_{s o, 3}-d_{s o, 2}\right)=k_{d_{s o, 2}}+D_{w, 2}-T-R_{g w}\end{array}$ & & \\
\hline WaterGAP2 & $\frac{\delta S_{s o}}{\delta t}=R_{i n}-R_{t o t}-E_{s o}$ & $R_{i n}$ & $R_{t o t} E_{s o}$ \\
\hline WAYS & $\frac{\partial S_{s o}}{\delta t}=M+P_{t h}-E_{s o}-R_{t o t}$ & $M P_{t h}$ & $E_{s o} R_{t o t}$ \\
\hline
\end{tabular}


Table S15. Total runoff in ISIMIP2b $R_{t o t, I S I M I P 2 b}$

\begin{tabular}{|c|c|}
\hline Model & Equation \\
\hline CLM4.5 & $R_{t o t, I S I M I P 2 b}=R_{s}+R_{s b}$ \\
\hline CLM5.0 & $R_{t o t, I S I M I P 2 b}=R_{s}+R_{s b}$ \\
\hline CWatM & $R_{t o t, I S I M I P 2 b}=R_{s}+R_{s b}$ \\
\hline $\mathrm{DBH}$ & $R_{t o t, I S I M I P 2 b}=R_{s}+R_{s b}$ \\
\hline H08 & $R_{t o t, I S I M I P 2 b}=R_{s}+R_{s b}$ \\
\hline JULES-W1 & $R_{t o t, I S I M I P 2 b}=R_{s}+R_{s b}$ \\
\hline LPJmL & $R_{t o t, I S I M I P 2 b}=R_{s}+R_{s b}$ \\
\hline Mac-PDM.20 & $R_{t o t, I S I M I P 2 b}=R_{s}+R_{s b}$ \\
\hline MATSIRO & $R_{t o t, I S I M I P 2 b}=R_{s}+R_{s b}$ \\
\hline mHM & $R_{t o t, I S I M I P 2 b}=R_{s}+R_{s b}$ \\
\hline MPI-HM & $R_{t o t, I S I M I P 2 b}=R_{s}+R_{s b}$ \\
\hline ORCHIDEE & $R_{t o t, I S I M I P 2 b}=R_{s}+R_{s b}$ \\
\hline PCR-GLOBWB & $R_{t o t, I S I M I P 2 b}=R_{s}+R_{s b}$ \\
\hline VIC & $R_{t o t, I S I M I P 2 b}=R_{s}+R_{s b}$ \\
\hline WaterGAP2 & $R_{t o t, I S I M I P 2 b}=R_{s}+R_{s b}$ \\
\hline WAYS & $R_{t o t, I S I M I P 2 b}=R_{s}+R_{s b}$ \\
\hline
\end{tabular}


Table S16. Surface runoff in ISIMIP $2 \mathrm{~b} R_{s}$

\begin{tabular}{ll}
\hline Model & Equation \\
\hline CLM4.5 & $R_{s}=R_{s u}$ \\
CLM5.0 & $R_{s}=R_{s u}$ \\
CWatM & $R_{s}=R_{s u}$ \\
DBH & $R_{s}=R_{s u}$ \\
H08 & $R_{s}=R_{s u}$ \\
JULES-W1 & $R_{s}=R_{s u}$ \\
LPJmL & $R_{s}=R_{s u}$ \\
Mac-PDM.20 & $R_{s}=R_{s u}$ \\
MATSIRO & $R_{s}=R_{s u}$ \\
mHM & $R_{s}=R_{s u}$ \\
MPI-HM & $R_{s}=R_{s u}$ \\
ORCHIDEE & $R_{s}=R_{s u}$ \\
PCR-GLOBWB & $R_{s}=R_{s u}$ \\
VIC & $R_{s}=R_{s u}$ \\
WaterGAP2 & $R_{s}=R_{s u}$ \\
WAYS & $R_{s}=R_{s u}$ \\
\hline
\end{tabular}


Table S17. Subsurface runoff in ISIMIP2b $R_{s b}$

\begin{tabular}{|c|c|}
\hline Model & Equation \\
\hline CLM4.5 & $R_{s b}=R_{g w}$ \\
\hline CLM5.0 & $R_{s b}=R_{g w}$ \\
\hline CWatM & $R_{s b}=R_{i f}+g w$ \\
\hline DBH & not represented \\
\hline H08 & $R_{s b}=R_{g w}$ \\
\hline JULES-W1 & $R_{s b}=R_{i f}$ \\
\hline LPJmL & $R_{s b}=R_{i f}$ \\
\hline Mac-PDM.20 & $R_{s b}=R_{g w}$ \\
\hline MATSIRO & $R_{s b}=R_{g w}$ \\
\hline $\mathrm{mHM}$ & $R_{s b}=R_{i f, f a s t}+R_{i f, s l o w}+R_{g w}$ \\
\hline MPI-HM & $R_{s b}=R_{g w r}$ \\
\hline ORCHIDEE & $R_{s b}=R_{g w}$ \\
\hline PCR-GLOBWB & $R_{s b}=R_{i f}$ \\
\hline \multirow[t]{2}{*}{ VIC } & $R_{s b}=$ \\
\hline & 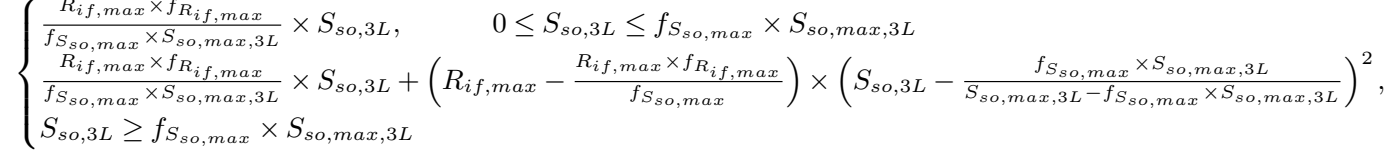 \\
\hline WaterGAP2 & $\hat{R}_{s b}=R_{g w}$ \\
\hline WAYS & $R_{s b}=R_{i f}+R_{g w}$ \\
\hline
\end{tabular}


Table S18. Total runoff from land $R_{t o t}$

\begin{tabular}{ll}
\hline Model & Equation \\
\hline CLM4.5 & not represented \\
CLM5.0 & not represented \\
CWatM & not represented \\
DBH & not represented \\
H08 & not represented \\
JULES-W1 & not represented \\
LPJmL & not represented \\
Mac-PDM.20 & not represented \\
MATSIRO & not represented \\
mHM & $R_{\text {tot }}=R_{s u}+R_{i f, f a s t}+R_{i f, s l o w}+R_{g w}$ \\
MPI-HM & not represented \\
ORCHIDEE & not represented \\
PCR-GLOBWB & not represented \\
VIC & not represented \\
WaterGAP2 & $R_{\text {tot }}=R_{\text {in }} \times\left(\frac{S_{s o}}{S_{s o, m a x}}\right)^{\gamma}+R_{b u}+R_{s a t}$ with $S_{s o, \text { max }}=S_{s o, T L} \times d_{s o, r o o t}$ and $R_{b u}=0.5 \times R_{i n} \times f_{b u}, R_{i n}$ \\
& is reduced by $R_{b u}$ before calculating $R_{t o t}, \gamma$ is the calibration parameter according to $[3,34]$. \\
WAYS & $R_{t o t}=\left(1-\left(1-\frac{S_{r z}}{(1+\beta) \times S_{r z, \text { max }}}\right)\right)^{\beta} \times R_{\text {in }}$ \\
\hline
\end{tabular}


Table S19. Runoff concentration in a grid cell

\begin{tabular}{ll}
\hline Model & Equation \\
\hline CLM4.5 & not represented \\
CLM5.0 & not represented \\
CWatM & $R_{G}^{n}=\sum_{L C} \times \sum_{f_{R}} \times \sum_{i}^{m a x} \times t r i_{x} \times R_{s u_{L C, R}} \times(t-i+1) ;$ for "i" runs from 1 to maximum number of days. \\
& trix $=\int_{i-1}^{i} \times \frac{2}{m a x}-\left|u ?-\frac{\max }{2}\right| \times \frac{4}{\max ^{2}} d u$ \\
& not represented \\
DBH & not represented \\
H08 & not represented \\
JULES-W1 & not represented \\
LPJmL & not represented \\
Mac-PDM.20 & not represented \\
MATSIRO & not represented \\
mHM & $R_{G}=\frac{S_{r v}}{\kappa_{o l}}$ \\
MPI-HM & not represented \\
ORCHIDEE & not represented \\
PCR-GLOBWB & not represented \\
VIC & not represented \\
WaterGAP2 & not represented \\
WAYS &
\end{tabular}


Table S20. Surface runoff $R_{s u}$

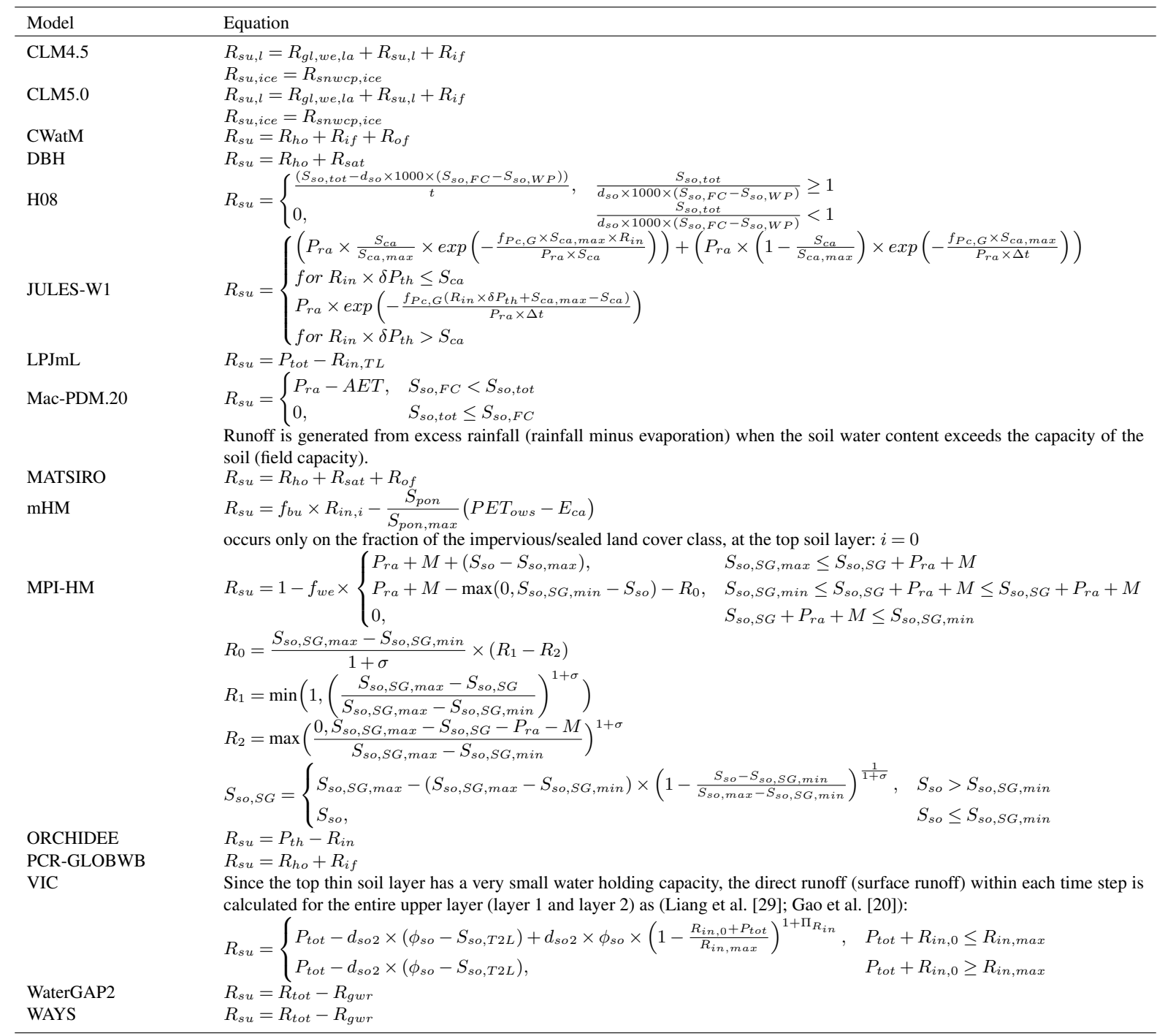


Table S21. Hortonian overland flow

\begin{tabular}{|c|c|}
\hline Model & Equation \\
\hline CLM4.5 & $R_{h o}=\max \left(R_{i n}-\left(1-f_{h 2 o}\right) R_{\text {in, max }}, 0\right)$ \\
\hline CLM5.0 & $R_{h o}=\max \left(R_{\text {in }}-\left(1-f_{h 2 o}\right) R_{\text {in }, \max }, 0\right)$ \\
\hline \multirow[t]{4}{*}{ CWatM } & $R_{h o}=M+P_{t h}-Q_{p f}-R_{i n}$ \\
\hline & Preferential flow, that bypass the soil matrix \\
\hline & $Q_{p f}=\left(P_{t h}+M\right) \times\left(\frac{S_{\text {so,rel }}}{S_{\text {so sat }}}\right)^{\beta}$ \\
\hline & $f_{G, \text { unsat }}$ (No,sat \\
\hline DBH & $R_{h o}=\int P_{t h} \times P_{\text {conv }}-k$ \\
\hline H08 & not represented \\
\hline JULES-W1 & not represented \\
\hline LPJmL & it is taken into account when surface runoff is computed, but not separately considered. \\
\hline Mac-PDM.20 & not represented \\
\hline MATSIRO & $\left.R_{\text {ho }}=B_{\text {stormy }} \times R_{\text {ho, stormy }}+\left(1-B_{\text {calm }}\right) \times R_{\text {ho, calm }}\right)$ \\
\hline $\mathrm{mHM}$ & not represented \\
\hline MPI-HM & not represented \\
\hline ORCHIDEE & not represented \\
\hline PCR-GLOBWB & $R_{h o}=1-\frac{S_{s o, s a t}-S_{s o, t o t}}{S_{s o t o t}-S_{s o W P}} \times P_{t o t}$ \\
\hline & $\begin{array}{c}S_{\text {so,tot }}-S_{\text {so }, W P} \\
R_{h o}=\max \left(R_{\text {in }}-\left(1-f_{h 2 o}\right) \times R_{\text {in }, \text { max }}, 0\right)\end{array}$ \\
\hline VIC & not represented \\
\hline WaterGAP2 & not represented \\
\hline WAYS & not represented \\
\hline
\end{tabular}


Table S22. Saturation excess overland flow

\begin{tabular}{|c|c|}
\hline Model & Equation \\
\hline CLM4.5 & not represented \\
\hline CLM5.0 & not represented \\
\hline \multirow[t]{2}{*}{ CWatM } & not represented \\
\hline & $f_{G, s a t}$ \\
\hline $\mathrm{DBH}$ & $R_{\text {sat }}=\int P_{t h}$ \\
\hline H08 & not represented \\
\hline JULES-W1 & $\begin{array}{l}\text { any saturation excess is moved to the soil layer below (the move of excess water to upper soil layers due to } \\
\text { saturation is restricted. The excess saturation water is forced down to lower layers, and if the bottom soil layer } \\
\text { becomes super-saturated, then the excess water is added to the interflow (Best et al. [5]). }\end{array}$ \\
\hline LPJmL & $R_{\text {sat }}=R_{\text {su }}$ \\
\hline Mac-PDM.20 & $R_{\text {sat }}=R_{s u}$ \\
\hline MATSIRO & $R_{s a t}=f_{G, s a t} \times f_{r i, s a t} \times P_{t h}$ \\
\hline mHM & not represented \\
\hline MPI-HM & implicitly included in surface runoff computation \\
\hline ORCHIDEE & not represented \\
\hline PCR-GLOBWB & not represented \\
\hline VIC & same as Table S20. \\
\hline \multirow[t]{2}{*}{ WaterGAP2 } & $R_{s a t}= \begin{cases}S_{s o}-S_{s o, \max }, & S_{s o}>S_{\text {so }, \max } \\
0, & \text { other cases }\end{cases}$ \\
\hline & $\begin{array}{l}\text { with } S_{\text {so, } \max }=d_{\text {so, root }} \times S_{\text {so }, T L} \\
\text { max }\left(0, S_{f}-S_{f t r}\right)\end{array}$ \\
\hline \multirow{2}{*}{ WAYS } & $R_{\text {sat }}=K_{f f}$ \\
\hline & $\begin{array}{l}\text { The Overflow of the uppermost layer is only active when the storage of the fast response reservoir exceeds the } \\
\text { specified threshold } S_{f t r}\end{array}$ \\
\hline
\end{tabular}


Table S23. Transpiration

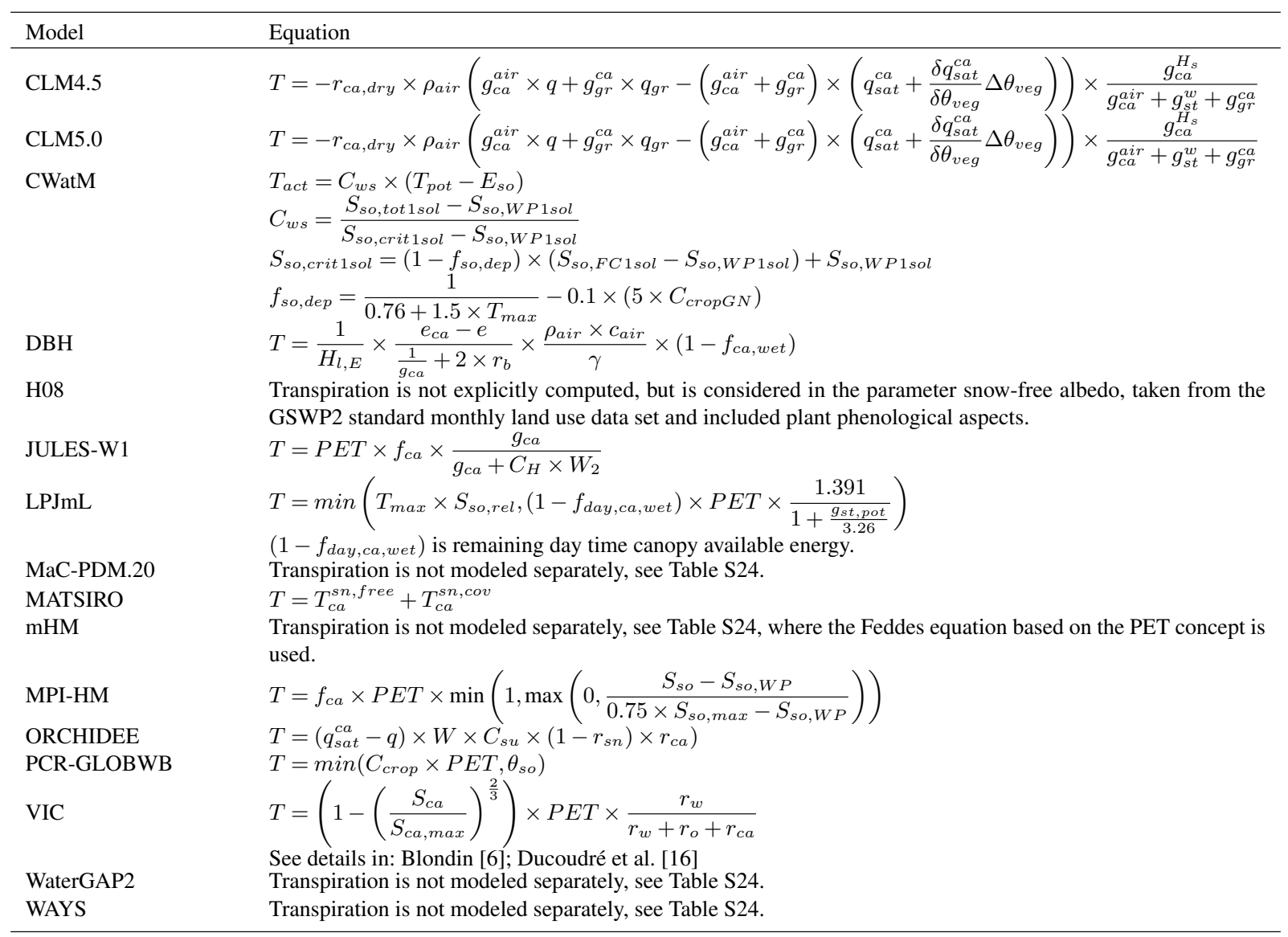


Table S24. Evaporation from soil $E_{s o}$

\begin{tabular}{|c|c|}
\hline Model & Equation \\
\hline CLM4.5 & $\begin{array}{l}E_{\text {so }}=-\frac{\rho_{\text {air }}\left(q-q_{\text {sat }}^{g r}\right)}{r_{\text {so }}} \\
E_{\text {so }}=-\frac{\rho_{\text {air }}\left(q-q_{\text {sat }}^{g r}\right)}{r_{\text {so }}}\end{array}$ \\
\hline $\begin{array}{l}\text { CWatM } \\
\text { DBH }\end{array}$ & $\begin{array}{l}E_{\text {so }}=C_{\text {crop }} \times P E T \\
E_{s o}=\frac{1}{H_{l, E}} \times \frac{q_{s o} \times\left(e_{c a}-e\right)}{r_{s o}+r_{w c a}} \times \frac{\rho_{\text {air }} \times c_{a i r}}{\gamma} \times\left(1-f_{s u, g r}\right)\end{array}$ \\
\hline JULES-W1 & $\begin{array}{l}E_{s o}=\alpha \times P E T \\
\text { where: } \\
\left\{\begin{array}{l}\alpha=1, \\
\alpha=\frac{S_{s o, t o t}}{S_{s o, c r i t}}, \quad S_{s o, t o t}<S_{\text {so, } \text { crit }}<S_{\text {so, crit }}\end{array}\right. \\
S_{\text {so,crit }}=0.75 \times S_{\text {so }, F C}\left(\text { fixed at } 150\left[\mathrm{kgm}^{-2}\right] \text {. For detail see [40]. }\right. \\
E_{\text {so }}=P E T \times\left(1-f_{a}\right) \times \frac{\left(1-f_{c a}\right) \times g_{s o}}{\left(1-f_{c a}\right) \times g_{s o}+C_{H} \times W_{2}} \\
g_{s o}=\frac{1}{100} \times\left(\frac{S_{s o, F L}}{S_{s o, c r i t}}\right)^{2}\end{array}$ \\
\hline $\begin{array}{l}\text { LPJmL } \\
\text { Mac-PDM.20 }\end{array}$ & $\begin{array}{l}E_{s o}=E_{\text {eq }} \times \alpha \times S_{s o, r e l} \times\left(1-f_{c a, G}\right) \\
E_{s o} \text { is assumed to occur at the } P E T \text { rate until } S_{s o, F C} \text { is reached, below which the ratio of } E_{s o} \text { to } P E T \\
\text { declines linearly to zero: } \\
\frac{E_{s o}}{P E T}=1, \quad S_{s o} \geq S_{s o, F C} \\
\frac{E_{s o}}{P E T}=\frac{S_{s o}}{S_{s o, F C}}, \quad S_{s o} \leq S_{s o, F C} \\
E_{s o}=E_{s o, l}^{s n, f r e e g r}\end{array}$ \\
\hline $\mathrm{mHM}$ & $\begin{array}{l}E T_{s o, i}= \begin{cases}\alpha \times f_{\text {root }, i} \times P E T^{\prime}, \\
\alpha \times f_{\text {root }, i} \times\left(P E T^{\prime}-\sum_{l=0}^{i-1} E T_{\text {so }, i-1}\right), & \text { for } i=0 \text { (first layer) }\end{cases} \\
P E T^{\prime}=P E T-E_{c a}-\frac{S_{\text {pon }}}{S_{\text {pon }, \text { max }}}\left(P E T_{\text {ows }}-E_{c a}\right) \\
\alpha= \begin{cases}0, & \frac{S_{s o, i}}{d_{s o, i}} \leq \phi_{r, i} \\
\frac{S_{s o, i}}{d_{s o, i}-\phi_{r, i}} & \phi_{r, i}<\frac{S_{s o, i}}{\phi_{s o, i}-\phi_{r, i}} \leq \phi_{w, i} \\
1, & \phi_{w, i}<\frac{S_{s o, i}}{d_{s o, i}}\end{cases} \end{array}$ \\
\hline MPI-HM & $E_{s o}=f_{s o, b a r e} \times P E T \times \min \left(1, \max \left(0, \frac{S_{\text {so }}-0.05 \times S_{\text {so, } \max }}{S_{\text {so }, \text { max }}-0.05 \times S_{\text {so }, \text { max }}}\right)\right)$ \\
\hline $\begin{array}{l}\text { ORCHIDEE } \\
\text { PCR-GLOBWB } \\
\text { VIC }\end{array}$ & $\begin{array}{l}E_{\text {so }}=\left(q_{s a t}^{c a}-q\right) \times W \times C_{s u} \times\left(1-r_{\text {floodplain }}\right) \times\left(1-r_{s n}\right) \times r_{s o} \\
E_{\text {so }}=C_{\text {crop }} \times P E T \\
\text { The bare soil evaporation only occurs on the top thin layer. When the surface soil is saturated, it evapo- } \\
\text { rates at the potential evaporation rate. When the top soil layer is not saturated, its evaporation rate (E1) } \\
\text { is calculated using the Arno formulation by Franchini and Pacciani [19]. }\end{array}$ \\
\hline & 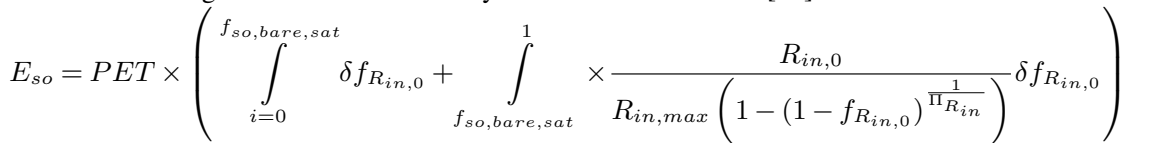 \\
\hline WaterGAP2 & $\begin{array}{l}E_{s o}=\min \left(P E T-E_{c a},\left(P E T_{, \max }-E_{c a}\right) \times \frac{S_{s o}}{S_{s o, \max }}\right) \\
P E T_{, \max }=15\left(\mathrm{~mm} \mathrm{day}^{-1}\right) \\
\text { Note: Evaporation and transpiration are calculated together. }\end{array}$ \\
\hline WAYS & $\begin{array}{l}E_{s o}=\left(P E T-E_{c a}\right) \times \min \left(1, \frac{S_{r z}}{v \times S_{r z, \max } \times(1+\beta)}\right) \\
\text { the scale parameter } v \text { is set to } 0.5 \text {, and the shape parameter } \beta \text { need to be fitted during the calibration } \\
\text { processes. } \\
\text { Note: Evaporation and transpiration are calculated together. }\end{array}$ \\
\hline
\end{tabular}


Table S25. Infiltration $R_{i n}$

\begin{tabular}{|c|c|}
\hline Model & Equation \\
\hline CLM4.5 & $\begin{array}{l}R_{\text {in }}=R_{\text {in }, \text { over }- \text { so }}+R_{\text {pe,h2osfc }} \\
R_{\text {in }, \text { over }- \text { so }}=\left(1-f_{h 2 o}\right) Q_{\text {in }, \text { surf }}-R_{h o}-\left(1-f_{\text {sn }}-f_{h 2 o}\right) E_{s o} \\
R_{\text {pe,h2osfc }}=\min \left(f_{h 2 o} R_{\text {in, } \max }, \frac{w_{s f c}}{\Delta t}\right)\end{array}$ \\
\hline CLM5.0 & $\begin{array}{l}R_{\text {in }}=R_{\text {in }, \text { over }- \text { so }}+R_{\text {pe,h2osfc }} \\
R_{\text {in }, \text { over }- \text { so }}=\left(1-f_{h 2 o}\right) Q_{\text {in }, \text { surf }}-R_{h o}-\left(1-f_{s n}-f_{h 2 o}\right) E_{s o} \\
R_{\text {pe, } h 2 o s f c}=\min \left(f_{h 2 o} R_{\text {in, } \max }, \frac{w_{s f c}}{\Delta t}\right)\end{array}$ \\
\hline CWatM & $\begin{array}{l}R_{\text {in }, \text { pot }}=\frac{S_{\text {so }, \text { sat }}}{\beta+1}-\frac{S_{\text {so }, \text { sat }}}{\beta+1} \times\left(1-(1-\alpha)^{\frac{\beta+1}{\beta}}\right) \\
\alpha=1-\left(1-\frac{S_{\text {so,rel }}}{S_{\text {so,sat }}}\right)^{\beta} \\
R_{\text {in }}=\min \left(P_{t o t}, R_{\text {in }, p o t}\right) \\
\text { The infiltration capacity of the soil is estimated using the Xinanjiang (also known as VIC/ARNO) model } \\
\beta=\text { shape parameter of the Xinanjiang model }\end{array}$ \\
\hline DBH & $R_{\text {in }}=k_{\text {sat }} \times\left(1+\frac{\left(S_{\text {so }, \text { sat }}-S_{\text {so }, \text { ini } i}\right) \times S_{\text {so }, \text { pot }}}{R_{\text {in }, \text { cum }}}\right)$ \\
\hline H08 & $\begin{array}{l}R_{i n}=\frac{S_{s o, F C}}{t} \times \frac{S_{s o, t o t}}{S_{s o, F C}} . \text { See }[21] \\
t=100[\text { days }]=86400 \times 100[\mathrm{~s}] ; \tau \text { is set at } 2 .\end{array}$ \\
\hline JULES-W1 & $R_{\text {in }}=k \times\left(\frac{\delta T}{\delta d_{s o}}+1\right) ; k=k_{\text {sat }}\left(\frac{\theta}{\theta_{s o}}\right)^{2 \times a+3}$ \\
\hline LPJmL & $R_{i n}=P_{t o t} \times \sqrt{1-\frac{S_{s o, t o t}-S_{s o, W P}}{S_{s o, s a t, i}-S_{s o, W P}}} \mathrm{i}=$ top layer \\
\hline Mac-PDM.20 & $R_{\text {in }}=P_{\text {tot }}-R_{\text {sat }}$ \\
\hline MATSIRO & $R_{i n}=P_{t h}-R_{h o}-R_{s a t}$ \\
\hline $\mathrm{mHM}$ & $R_{i n, i}= \begin{cases}P_{t h}+M-R_{s u}, & \mathrm{i}=0 \text { (top soil layer) } \\
R_{i n, i-1}-\left(\frac{\frac{S_{s o, i}}{d_{s o, i}}-\phi_{r, i}}{\phi_{s, i}-\phi_{r, i}}\right)^{a_{i}}, & i>0\end{cases}$ \\
\hline $\begin{array}{l}\text { MPI-HM } \\
\text { ORCHIDEE }\end{array}$ & $\begin{array}{l}R_{i n}=\left(P_{r a}+M\right) \times\left(1-f_{w e}\right)-R_{s u} \\
R_{i n, j}=\frac{k_{j, j}+k_{s a t, j}}{2}\end{array}$ \\
\hline $\begin{array}{l}\text { PCR-GLOBWB } \\
\text { VIC }\end{array}$ & $\begin{array}{l}R_{i n}=\min \left(P_{r a}^{2}+M, k_{s a t}\right) \\
\text { for there is no vegetation coverage: } \\
R_{i n}=P_{r a}-R_{p e} \\
\text { for there is vegetation coverage: } \\
R_{i n}=P_{t h}-R_{p e} \\
\text { See [20]: }[29]\end{array}$ \\
\hline $\begin{array}{l}\text { WaterGAP2 } \\
\text { WAYS }\end{array}$ & $\begin{array}{l}R_{\text {in }}=M+P_{t h}-\left(0.5 \times\left(M+P_{t h}\right) \times f_{b u}\right) \\
R_{\text {in }}=M+P_{t h}\end{array}$ \\
\hline
\end{tabular}


Table S26. Interflow

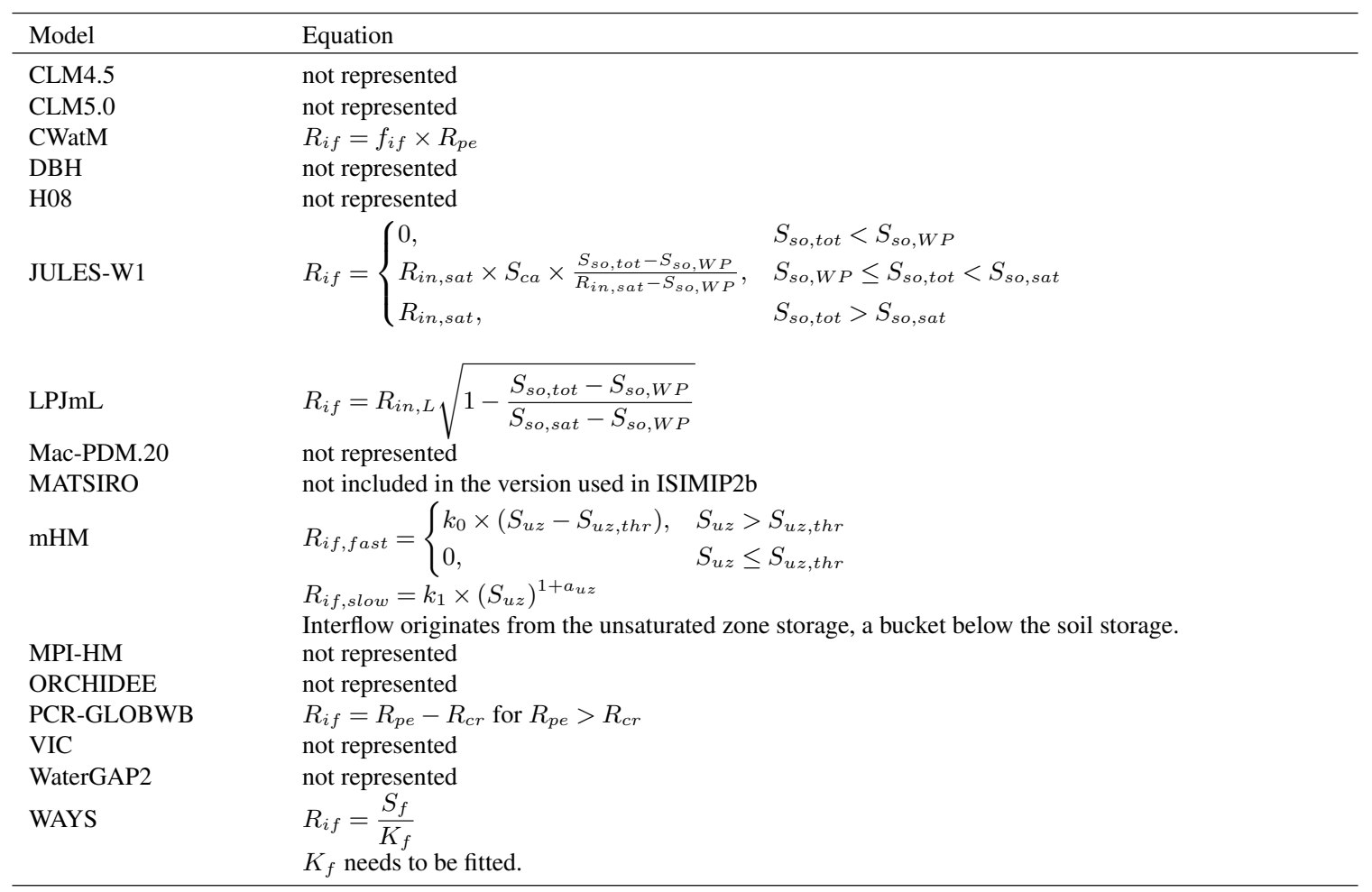


Table S27. Percolation $R_{p e}$

\begin{tabular}{|c|c|}
\hline Model & Equation \\
\hline CLM4.5 & not represented \\
\hline CLM5.0 & not represented \\
\hline CWatM & $R_{p e}=\left(1-f_{w e}\right) \times \min \left(R_{i n}, k_{3 s o}\right)$ \\
\hline $\begin{array}{l}\text { DBH } \\
\text { H08 } \\
\text { JULES-W1 }\end{array}$ & $\begin{array}{l}\text { Unsaturated conductivity using Van Genuchten equation for each soil layer } \\
\text { not represented } \\
\text { not represented } \\
\text { not represented }\end{array}$ \\
\hline LPJmL & $R_{p e}=\left(S_{s o, s a t}-S_{s o, F C}\right) \times\left[1-\exp \left(\frac{-\Delta t}{\frac{S_{s o, F C}}{k}}\right)\right]$ \\
\hline $\begin{array}{l}\text { Mac- } \\
\text { PDM.20 }\end{array}$ & not represented \\
\hline MATSIRO & not explicitly represented \\
\hline $\mathrm{mHM}$ & not represented (defined as groundwater recharge, see Table S30) \\
\hline MPI-HM & not represented \\
\hline ORCHIDEE & not represented \\
\hline $\begin{array}{l}\text { PCR- } \\
\text { GLOBWB }\end{array}$ & not represented \\
\hline VIC & not represented \\
\hline WaterGAP2 & not represented \\
\hline WAYS & not explicitly represented \\
\hline
\end{tabular}


Table S28. Capillary rise $R_{c r}$

\begin{tabular}{ll}
\hline Model & Equation \\
\hline CLM4.5 & Represented through the concept of soil matrix potential: \\
& $\Psi_{i}=\Psi_{s a t, i}\left(\frac{S_{s o, t o t}}{S_{s o, s a t}}\right)^{C_{s o, i}} \geq-1 \times 10^{8}$ \\
CLM5.0 & Represented through the concept of soil matrix potential: \\
& $\Psi_{i}=\Psi_{s a t, i}\left(\frac{S_{s o, t o t}}{S_{s o, s a t}}\right)^{C_{s o, i}} \geq-1 \times 10^{8}$ \\
CWatM & $R_{c r}=0.5 \times \sqrt{k_{3 s o} \times k_{s a t}} \times R_{c r, m a x}$ \\
& $R_{c r, \text { max }}:$ cell fraction, depending on height of ground water table and relative elevation of grid \\
DBH & not represented \\
H08 & not represented \\
JULES-W1 & not represented \\
LPJmL & not represented \\
Mac-PDM.20 & not represented \\
MATSIRO & $R_{c r}=k \times\left(\frac{\delta Z_{c r}}{\delta Z}-1\right)$ \\
mHM & not represented \\
MPI-HM & not represented \\
ORCHIDEE & not represented \\
PCR-GLOBWB & $R_{c r}=0.5 \times \sqrt{k_{3 s o} \times k_{s a t}} \times R_{c r, m a x}$ \\
VIC & $R_{c r, \text { max }}:$ cell fraction, depending on height of ground water table and relative elevation of grid \\
WaterGAP2 & not represented \\
WAYS & not represented \\
\hline
\end{tabular}


Table S29. Groundwater storage $S_{g w}$

\begin{tabular}{|c|c|c|c|}
\hline \multirow[t]{2}{*}{ Model } & \multirow[t]{2}{*}{ Equation } & \multicolumn{2}{|c|}{ Water Flux } \\
\hline & & Inflows & Outflows \\
\hline CLM4.5 & $\begin{array}{l}\text { Only represented indirectly through changes in the water table } \\
\text { depth (unconfined aquifer) } \\
\Delta d_{w t}=\frac{\left(R_{g w r}-R_{g w}\right)}{S_{y}} \Delta t\end{array}$ & $R_{g w r}$ & $R_{g w}$ \\
\hline CLM5.0 & $\begin{array}{l}\text { Only represented indirectly through changes in the water table } \\
\text { depth (unconfined aquifer) } \\
\Delta d_{w t}=\frac{\left(R_{g w r}-R_{g w}\right)}{S_{y}} \Delta t\end{array}$ & $R_{g w r}$ & $R_{g w}$ \\
\hline CWatM & $\frac{\delta S_{g w}}{\delta t}=Q_{p f}+R_{p e}-R_{c r}-R_{g w}-A_{i r r}^{g w}-A_{d o m}^{g w}-A_{m a n}^{g w}-A_{l i v}^{g w}$ & $R_{p e} Q_{p f}$ & $\begin{array}{l}R_{c r} R_{g w} \\
A_{i r r}^{g w} A_{d o m}^{g w} \\
A_{\text {man }}^{g w} A_{\text {liv }}^{g w}\end{array}$ \\
\hline DBH & not represented & & \\
\hline H08 & $\begin{array}{l}\frac{\delta S_{g w}}{\delta t}=S_{g w, r w}-S_{g w, n r w} \\
S_{g w, r w}=R_{g w r}-R_{g w}-\frac{A_{t o t}}{B_{G}}\end{array}$ & $R_{g w r}$ & $R_{g w} A_{t o t}$ \\
\hline $\begin{array}{l}\text { JULES-W1 } \\
\text { LPJmL }\end{array}$ & $\begin{array}{l}\text { not represented } \\
\text { not represented }\end{array}$ & & \\
\hline Mac-PDM.20 & $\frac{\delta S_{g w}}{\delta t}=R_{g w r}-R_{g w}$ & $R_{g w r}$ & $R_{g w}$ \\
\hline MATSIRO & $\begin{array}{l}\frac{\delta S_{g w}}{\delta t}=\sum_{n=1,13}\left(\left(S_{s o, F_{i}}+S_{s o, u F_{i}}\right) \times d_{s o}\right) \\
\text { It explicitly diagnoses groundwater table depth and calculate water flux between } \\
\text { groundwater storage and unsaturated soil based on the condition of the soil layer } \\
\text { that has groundwater table. Groundwater pumping and baseflow are used in- } \\
\text { stead of root uptake and interflow. }\end{array}$ & & \\
\hline $\mathrm{mHM}$ & $\begin{array}{l}\text { no water table depth or confined aquifers are considered: } \\
\frac{\delta S_{g w}}{\delta t}=R_{g w r}-R_{g w}\end{array}$ & $R_{g w r}$ & $R_{g w}$ \\
\hline ORCHIDEE & $\begin{array}{l}\text { very simple representation, no water table depth or confined aquifers are con- } \\
\text { sidered } \\
\frac{\delta S_{g w}}{\delta t}=R_{g w r}-R_{g w}-f_{i r r} \times S_{g w} \\
\text { not represented }\end{array}$ & $R_{g w r}$ & $R_{g w}$ \\
\hline PCR-GLOBWB & $\frac{\delta S_{g w}}{\delta t}=R_{p e}-R_{c r}-R_{g w}-A_{i r r}^{g w}-A_{d o m}^{g w}-A_{m a n}^{g w}-A_{l i v}^{g w}$ & $R_{p e}$ & $\begin{array}{l}R_{c r} R_{g w} \\
A_{i r r}^{g w} A_{d o m}^{g w} \\
A_{\text {man }}^{g w} A_{\text {liv }}^{g w}\end{array}$ \\
\hline VIC & not represented & & \\
\hline WaterGAP2 & $\frac{\delta S_{g w}}{\delta t}=R_{g w r}-R_{g w}-A_{g w}$ & $R_{g w r}$ & $R_{g w} A_{g w}$ \\
\hline WAYS & $\frac{\delta S_{g w}}{\delta t}=R_{g w r}-R_{g w}$ & $R_{g w r}$ & $R_{g w}$ \\
\hline
\end{tabular}


Table S30. Groundwater recharge $R_{g w r}$

\begin{tabular}{|c|c|}
\hline Model & Equation \\
\hline CLM4.5 & $R_{g w r}=-k_{a q} \frac{\left(\Psi_{w t}-\Psi_{j w t}\right)}{\left(d_{w t}-d_{w t, i}\right)}$ \\
\hline CLM5.0 & $R_{g w r}=-k_{a q} \frac{\left(\Psi_{w t}-\Psi_{j w t}\right)}{\left(d_{w t}-d_{w t, i}\right)}$ \\
\hline CWatM & $R_{g w r}=R_{p e}+Q_{p f}-R_{c r}$ \\
\hline $\mathrm{DBH}$ & $R_{g w r}=k_{3 s o} \times\left(\frac{\delta S_{s o, \max , 3 L}}{\delta d_{s o, 3}}+1\right)$ \\
\hline $\begin{array}{l}\text { H08 } \\
\text { JULES-W1 }\end{array}$ & $\begin{array}{l}R_{g w r}=\min \left(R_{g w r, \max }, f_{r} \times f_{s o, t e x} \times f_{h g} \times f_{p g} \times R_{t o t}\right) . \text { See [11] } \\
\text { not represented }\end{array}$ \\
\hline LPJmL & $R_{g w r}=R_{i n, B L} \sqrt{1-\frac{S_{s o, t o t}-S_{s o, W P}}{S_{s o, s a t}-S_{s o, W P}}}$ \\
\hline Mac-PDM.20 & $R_{g w r}=S_{s o}-S_{s o, F C}^{\prime}$ \\
\hline $\begin{array}{l}\text { MATSIRO } \\
\text { mHM }\end{array}$ & $\begin{array}{l}R_{g w r}=\frac{\delta S_{s o, u F_{i, g w}}}{\Delta t} \\
R_{g w r}=k_{u z} S_{u z}\end{array}$ \\
\hline MPI-HM & $\begin{array}{l}R_{g w r}=\left(1-f_{w e}\right) \times R_{g w r, \text { min }} \times \frac{S_{s o}}{S_{s o, \max }}+\left(R_{g w r, \max }-R_{g w r, \min }\right) \times\left(\min \left(1, \max \left(0, \frac{S_{s o}-0.90 \times S_{s o, \max }}{S_{s o, \max }-0.90 \times S_{s o, \max }}\right)\right)^{1.5}\right) \\
\text { if } S_{s o}>0.05 \times S_{s o, \max }\end{array}$ \\
\hline ORCHIDEE & $R_{g w r}=\max \left(0,1-\frac{S_{\text {so }}}{S_{\text {somax }}}\right) \times R_{g w}$ See $[15]$ \\
\hline $\begin{array}{l}\text { PCR-GLOBWB } \\
\text { VIC }\end{array}$ & $\begin{array}{l}R_{g w r}=R_{p e}-R_{c r} \\
\text { not represented }\end{array}$ \\
\hline WaterGAP2 & $R_{g w r}= \begin{cases}0 & (\text { semi }) \text { arid grid cell, sandy texture and } P_{t h} \leq 12.5\left(\mathrm{~mm} \mathrm{day}^{-1}\right) \\
\min \left(R_{g w r, \max }, f_{r} \times f_{s o, t e x} \times f_{h g} \times f_{p g} \times R_{t o t}\right) & \text { other cases }\end{cases}$ \\
\hline WAYS & $\begin{array}{l}\text { where } R_{g w r, \max } \text { is set to } 7,4.5,2.5 \mathrm{~mm} \mathrm{~d}^{-1} \text { for sandy, loamy, and clayey soils. See [11] } \\
R_{g w r}=\min \left(R_{g w r, \max }, f_{r} \times f_{s o, t e x} \times f_{h g} \times f_{p g} \times R_{t o t}\right) \text {. See [11] }\end{array}$ \\
\hline
\end{tabular}


Table S31. Groundwater runoff $R_{g w}$

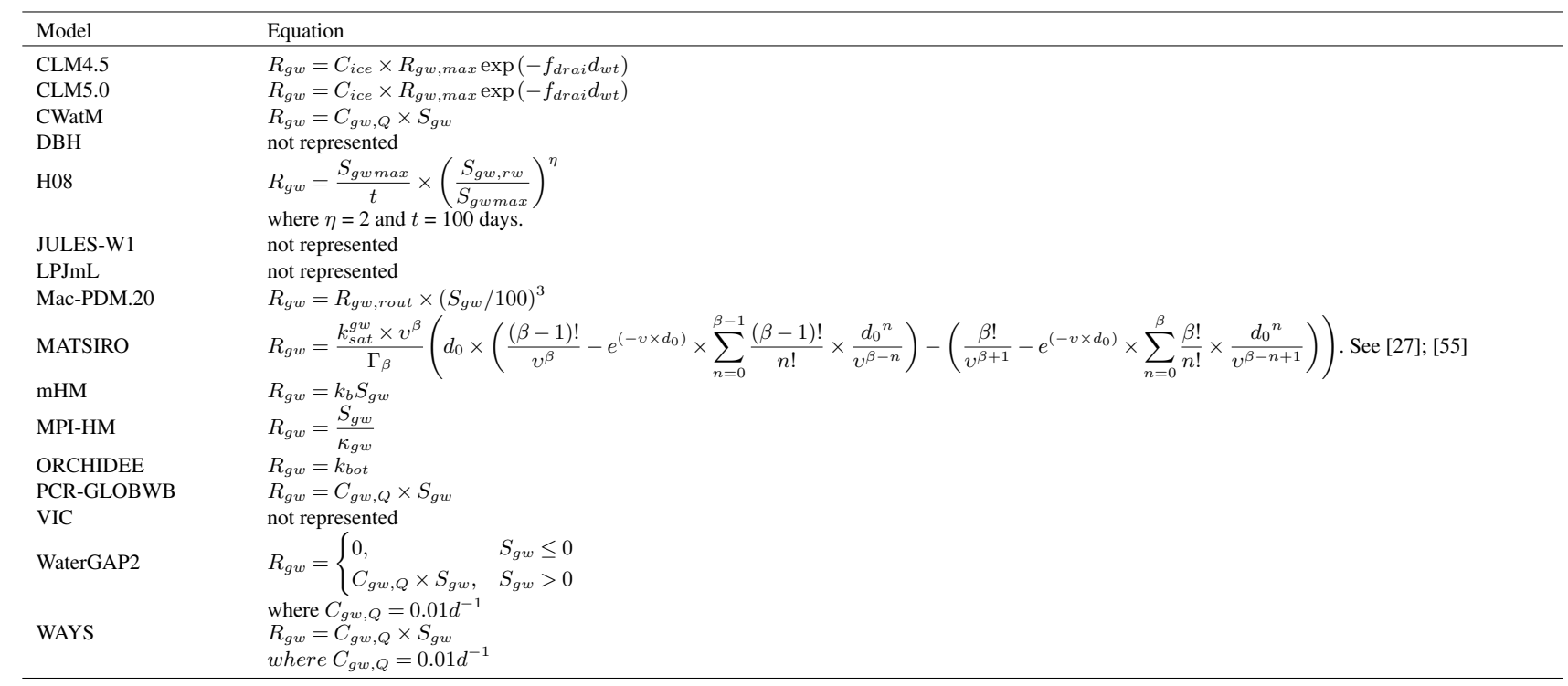


Table S32. Lake storage $S_{l a}$

\begin{tabular}{|c|c|c|c|}
\hline \multirow[t]{2}{*}{ Model } & \multirow[t]{2}{*}{ Equation } & \multicolumn{2}{|c|}{ Water Flux } \\
\hline & & Inflows & Outflows \\
\hline CLM4.5 & virtual storage, i.e. P-E automatically balanced by lake runoff term & & \\
\hline CLM5.0 & virtual storage, i.e. P-E automatically balanced by lake runoff term & & \\
\hline CWatM & $\begin{array}{l}\text { it has two types of lakes: "global lakes" are lakes that receive inflow not only from the grid cell } \\
\text { itself and "local lakes" receive inflow from the grid cell itself. } \\
\frac{\delta S_{l a}}{\delta t}=Q_{r i, \text { in }}-Q_{r i, \text { out }}\end{array}$ & $Q_{r i, i n}$ & $Q_{\text {ri,out }}$ \\
\hline DBH & not represented & & \\
\hline H08 & not represented & & \\
\hline JULES-W1 & not represented & & \\
\hline LPJmL & $\frac{\delta S_{l a}}{\delta t}=P_{t o t}+Q_{i u}-Q_{l a}-E_{l a}-A$ & $P_{t o t}, Q_{i u}$ & $E_{l a}, A$ \\
\hline Mac-PDM.20 & not represented & & \\
\hline $\begin{array}{l}\text { MATSIRO } \\
\text { mHM }\end{array}$ & $\begin{array}{l}\text { Lake storage is a part of river storage. Not explicitly included in the version used for ISIMIP } 2 b \text {. } \\
\text { not represented }\end{array}$ & & \\
\hline MPI-HM & Lake storage is part of the wetland storage. & & \\
\hline ORCHIDEE & not represented & & \\
\hline PCR-GLOBWB & Lake storage is a part of river storage. & & \\
\hline VIC & not represented & & \\
\hline \multirow[t]{5}{*}{ WaterGAP2 } & $\begin{array}{l}\text { WaterGAP2 has two types of representations of lakes. Local lakes receive water only from } \\
\text { the grid cell itself, while global lakes receive water from grid cell itself and the inflow from } \\
\text { upstream cells. } \\
\frac{\delta S_{l a, l o c a l}}{\delta t}=\left(R_{s u} \times f_{s w b}\right)+R_{g w}+A_{r f}+B_{l a, l o c a l} \times\left(P_{t o t}-E_{l a}\right)-R_{g w r}^{s w b}-A_{l a}-Q_{l a, l o c a l} \\
B_{l a, l o c a l}=C_{B, \text { red }} \times B_{l a, l o c a l, \text { max }} \\
C_{B, \text { red }}=1-\left(\frac{\left|S_{l a, l o c a l}-S_{l a, \text { local }, \text { max }}\right|}{2 S_{l a, l o c a l, \text { max }}}\right)^{3.32}\end{array}$ & $\begin{array}{l}R_{s u}, R_{g w} \\
P_{t o t}, Q_{i u} \\
Q_{w e, l o c a l} \\
A_{r f}\end{array}$ & $\begin{array}{l}R_{g w r}^{s w b}, A_{l a}, \\
Q_{l a, l o c a l} \\
Q_{l a, g l o b a l} \\
E_{l a}\end{array}$ \\
\hline & 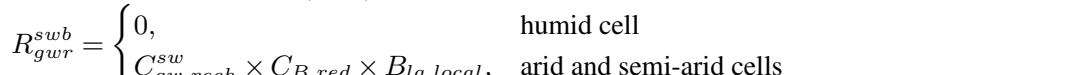 & & \\
\hline & $\begin{array}{l}\frac{\delta S_{l a, \text { global }}}{\delta t}=Q_{i u}+A_{r f}+Q_{w e, l o c a l}+B_{l a, g l o b a l} \times\left(P_{t o t}-E_{l a}\right)-R_{g w r}^{s w b}-A_{l a}-Q_{l a, g l o b a l} \\
B_{l a, \text { global }}=C_{B, \text { red }} \times B_{l a, \text { global }, \text { max }} \\
C_{B, \text { red }}=1-\left(\frac{\left|S_{l a, \text { global }}-S_{\text {la,global } \text { max }}\right|}{2 S_{\text {la,global }, \text { max }}}\right)^{3.32}\end{array}$ & & \\
\hline & $R_{g w r}^{s w b}= \begin{cases}0, & \text { humid cell } \\
C_{g w, \text { rech }}^{s w} \times C_{B, \text { red }} \times B_{l a, \text { global }}, & \text { arid and semi-arid cells }\end{cases}$ & & \\
\hline & not represented & & \\
\hline
\end{tabular}


Table S33. Evaporation from lake $E_{l a}$

\begin{tabular}{ll}
\hline Model & Equation \\
\hline CLM4.5 & not represented \\
CLM5.0 & not represented \\
CWatM & $E_{l a}=\min \left(S_{l a}, E_{l a, p o t}-\min \left(P_{t o t}, E_{l a, p o t}\right)\right.$ \\
DBH & not represented \\
H08 & not represented \\
JULES-W1 & not represented \\
LPJmL & $E_{l a}=\min \left(S_{l a}, E_{l a, p o t}\right)$ \\
Mac-PDM.20 & $E_{l a}=P_{t o t}-P E T$ \\
MATSIRO & not represented \\
mHM & not represented \\
MPI-HM & not represented \\
ORCHIDEE & not represented \\
PCR-GLOBWB & $E_{l a}=P E T$ \\
VIC & not represented \\
WaterGAP2 & $E_{l a}=P E T$ with albedo $=0.08$ \\
WAYS & not represented \\
\hline
\end{tabular}


Table S34. Outflow from lake $Q_{l a}$

\begin{tabular}{ll}
\hline Model & Equation \\
\hline CLM4.5 & not represented \\
CLM5.0 & not represented \\
CWatM & Modified Puls approach (Chow et al. [9]; Maniak [30]). Routing, reservoirs and \\
& $\frac{S_{l a 1}+S_{l a 2}}{\Delta t}=\frac{Q_{i u, l a 1}+Q_{\text {iu }, l a 2}}{2}-\frac{Q_{l a 1}+Q_{l a 2}}{2}$ \\
& for: $1=$ first time step $(t) ; 2=$ second time step $(t+\Delta t)$ \\
DBH & not represented \\
H08 & not represented \\
JULES-W1 & not represented \\
LPJmL & for $S_{l a} \geq S_{l a, \text { max }}, Q_{l a}=\left(S_{l a, \text { max }}-S_{l a}\right)$, else $Q_{l a}=0$ \\
Mac-PDM.20 & not represented \\
MATSIRO & not included in the version used for ISIMIP2b \\
mHM & not represented \\
MPI-HM & not represented \\
ORCHIDEE & not represented \\
PCR-GLOBWB & $Q_{l a}=1.7 \times C_{w e i r} \times$ max $\left(h_{w}-h_{o f}\right)^{1.5} \times b_{w e i r}$ \\
VIC & not represented \\
WaterGAP2 & $Q_{l a, l o c a l}=C_{\text {sw,out }} \times S_{l a, l o c a l} \times\left(\frac{S_{l a, l o c a l}}{S_{l a, l o c a l, m a x}}\right)^{1.5}$ \\
& $Q_{l a, g l o b a l}=C_{\text {sw }, \text { out }} \times S_{l a, g l o b a l}$ \\
WAYS & for $C_{\text {sw }, \text { out }}=0.01 d^{-1}$ \\
\hline
\end{tabular}


Table S35. Reservoir storage $S_{r e}$

\begin{tabular}{|c|c|c|c|}
\hline \multirow[t]{2}{*}{ Model } & \multirow[t]{2}{*}{ Equation } & \multicolumn{2}{|l|}{ Water Flux } \\
\hline & & Inflows & Outflows \\
\hline CLM4.5 & not represented & & \\
\hline CLM5.0 & not represented & & \\
\hline CWatM & $\frac{\delta S_{r e}}{\delta t}=Q_{r i, i n}-Q_{r i, o u t}$ & $Q_{r i, i n}$ & $Q_{\text {ri,out }}$ \\
\hline DBH & not represented & & \\
\hline JULES-W1 & $\begin{array}{l}\frac{\delta S_{\text {relocal }}}{\delta t}=R_{\text {tot }} \times B_{r e}-A_{r e}-Q_{r e} \\
\frac{\delta S_{r e g l o b a l}}{\delta t}=Q_{r i, \text { in }}-Q_{r i, \text { out }} \\
\text { not represented }\end{array}$ & $R_{t o t} Q_{r i, i n}$ & $\begin{array}{l}A_{\text {re }} \quad R_{\text {tot }} \\
Q_{\text {ri,out }}\end{array}$ \\
\hline $\begin{array}{l}\text { LPJmL } \\
\text { Mac-PDM.20 }\end{array}$ & $\begin{array}{l}\frac{\delta S_{r e}}{\delta t}=P_{t o t}+Q_{i u}-Q_{l a}-E_{r e}-A \\
\text { not represented }\end{array}$ & $P_{t o t} Q_{i u}$ & $Q_{l a} E_{r e} A$ \\
\hline MATSIRO & $\begin{array}{l}\frac{\delta S_{r e}}{\delta t}=S_{\text {reglobal }}+S_{\text {relocal }} \\
\frac{\delta S_{r e l o c a l}}{\delta t}=R_{t o t}-\frac{Q_{r e, l o c a l}}{B}-A_{d o m}^{\text {pond }}-A_{\text {ind }}^{\text {pond }}-A_{\text {irr }}^{\text {pond }} \\
\frac{\delta S_{r e g l o b a l}}{\delta t}=\frac{Q_{i u}+Q_{r e, l o c a l}-Q_{r e}}{B}-A_{\text {dom }}^{r e, g l o b a l}-A_{\text {ind }}^{r e, g l o b a l}-A_{i r r}^{r e, g l o b a l}\end{array}$ & $Q_{i u, r e}$ & $\begin{array}{l}Q_{r e} A_{\text {dom }} \\
A_{\text {irr }} A_{\text {ind }}\end{array}$ \\
\hline $\begin{array}{l}\text { mHM } \\
\text { MPI-HM } \\
\text { ORCHIDEE }\end{array}$ & $\begin{array}{l}\text { not represented } \\
\text { not represented } \\
\text { not represented }\end{array}$ & & \\
\hline PCR-GLOBWB & $\frac{\delta S_{r e}}{\delta t}=\left\{\begin{array}{l}Q_{r i}=Q_{\text {mean }} \text { for } S_{r e}>0.7 \times S_{r e, C} \\
Q_{r i}=\max \left(Q_{\text {mean }} \times \frac{S_{r e, C}-S_{r e, a c t}}{S_{r e, a c t}-0.3 \times S_{r e, C}}, Q_{e f}\right) \text { for } 0.3>S_{r e}>0.7 \times S_{r e, C} \\
Q_{e f} \text { for } 0.3<S_{r e}\end{array}\right.$ & & \\
\hline VIC & not represented & & \\
\hline WaterGAP2 & $\begin{array}{l}\frac{\delta S_{r e}}{\delta t}=Q_{i u}+A_{r f}+Q_{w e, l o c a l}+B_{r e} \times\left(P_{t o t}-E_{r e}\right)-R_{g w r}^{s w b}-A_{r e}-Q_{r e} \\
\text { for } B_{r e}=C_{B, r e d} \times B_{r e, \text { max }} \\
\text { for } C_{B, r e d}=1-\left(\frac{\left|S_{r e}-S_{r e, \max }\right|}{S_{r e, \text { max }}}\right)^{2.814}\end{array}$ & $\begin{array}{l}Q_{i u}, Q_{w e, l o c a l} \\
P_{t o t}, A_{r f}\end{array}$ & $\begin{array}{l}E_{r e}, R_{g w r}^{s w b}, \\
B_{r e}, Q_{r e}, \\
A_{r e}\end{array}$ \\
\hline & for $R_{g w r}^{s w b}= \begin{cases}0 & \text { for humid cell } \\
C_{g w, \text { rech }}^{s w} \times C_{B, \text { red }} \times B_{r e} & \text { for arid and semi-arid cells }\end{cases}$ & & \\
\hline WAYS & not represented & & \\
\hline
\end{tabular}


Table S36. Inflow from upstream surface water bodies for reservoir storage

\begin{tabular}{ll}
\hline Model & Equation \\
\hline CLM4.5 & not represented \\
CLM5.0 & not represented \\
CWatM & sum of inflows of water from neighboring upstream grid cells routed with kinematic wave approach \\
DBH & not represented \\
H08 & not represented \\
JULES-W1 & not represented \\
LPJmL & not represented \\
Mac-PDM.20 & not represented \\
MATSIRO & not represented \\
mHM & not represented \\
MPI-HM & not represented \\
ORCHIDEE & not represented \\
PCR-GLOBWB & sum of inflows of water from neighboring upstream grid cells routed with kinematic wave approach \\
VIC & not represented \\
WaterGAP2 & not represented \\
WAYS & not represented \\
\hline
\end{tabular}


Table S37. Outflow from reservoir $Q_{r e}$

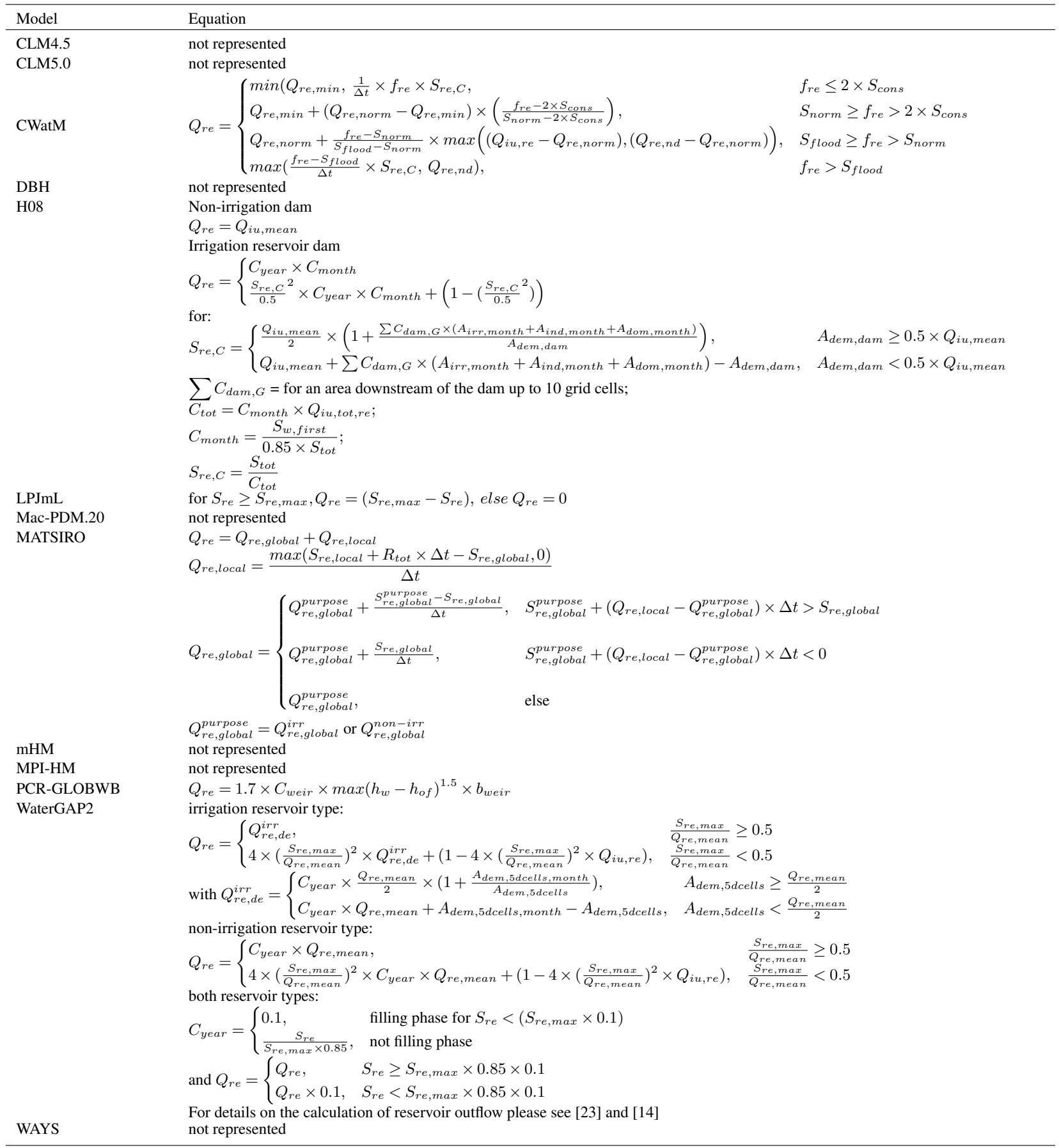


Table S38. Evaporation from reservoir $E_{r e}$

\begin{tabular}{ll}
\hline Model & Equation \\
\hline CLM4.5 & not represented \\
CLM5.0 & not represented \\
CWatM & $E_{r e}=\min \left(S_{r e}, E_{r e, p o t}-\min \left(P_{t o t}, E_{r e, p o t}\right) \times B_{r e}\right.$ \\
DBH & not represented \\
H08 & not represented \\
JULES-W1 & not represented \\
LPJmL & $E_{r e}=\min \left(S_{r e}, E_{r e, p o t}\right)$ \\
Mac-PDM.20 & not represented \\
MATSIRO & not represented \\
mHM & not represented \\
MPI-HM & not represented \\
ORCHIDEE & not represented \\
PCR-GLOBWB & $E_{r e}=P E T$ \\
VIC & not represented \\
WaterGAP2 & $E_{r e}=P E T$ with albedo $=0.08$ \\
WAYS & not represented \\
\hline
\end{tabular}


Table S39. Wetland storage $S_{w e}$

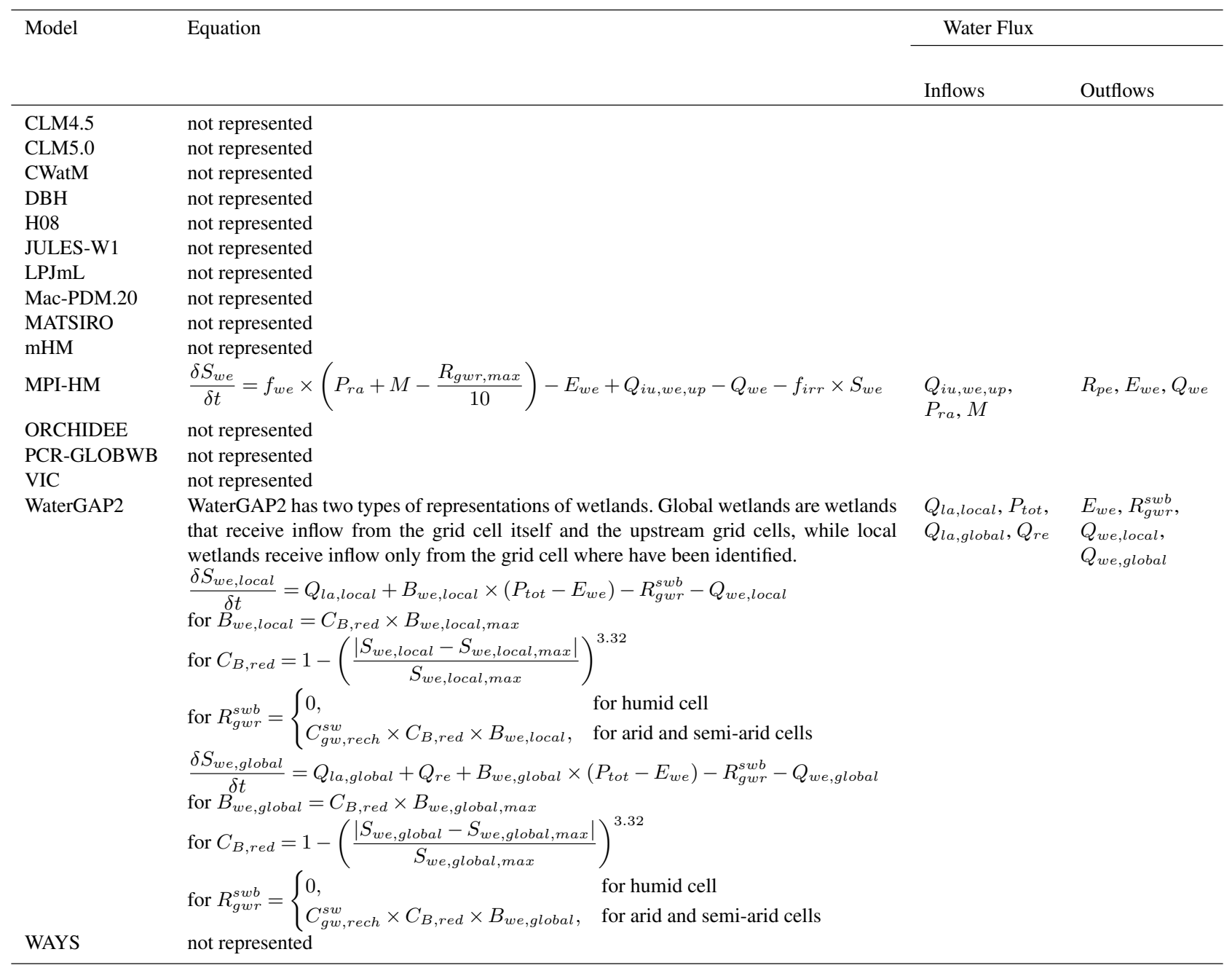


Table S40. Inflow from upstream grid cell for wetland storage $Q_{i u, w e, u p}$

\begin{tabular}{ll}
\hline Model & Equation \\
\hline CLM4.5 & not represented \\
CLM5.0 & not represented \\
CWatM & not represented \\
DBH & not represented \\
H08 & not represented \\
JULES-W1 & not represented \\
LPJmL & not represented \\
Mac-PDM.20 & not represented \\
MATSIRO & not represented \\
mHM & not represented \\
MPI-Hm & $Q_{\text {iu, we,up }}=f_{w e}{ }^{2} \times\left(Q_{r v, u p}+R_{g w, u p}+Q_{r i, u p}\right)$ \\
ORCHIDEE & not represented \\
PCR-GLOBWB & not represented \\
VIC & not represented \\
WaterGAP2 & not represented \\
WAYS & not represented \\
\hline
\end{tabular}


Table S41. Evaporation from wetland $E_{w e}$

\begin{tabular}{ll}
\hline Model & Equation \\
\hline CLM4.5 & not represented \\
CLM5.0 & not represented \\
CWatM & not represented \\
DBH & not represented \\
H08 & not represented \\
JULES-W1 & not represented \\
LPJmL & not represented \\
Mac-PDM.20 & not represented \\
MATSIRO & not represented \\
mHM & not represented \\
MPI-HM & $E_{w e}=P E T \times f_{w e}$ \\
ORCHIDEE & not represented \\
PCR-GLOBWB & not represented \\
VIC & not represented \\
WaterGAP2 & $E_{w e}=P E T$ with albedo $=0.08$ \\
WAYS & not represented \\
\hline
\end{tabular}


Table S42. Outflow from wetland $Q_{w e}$

\begin{tabular}{|c|c|}
\hline Model & Equation \\
\hline CLM4.5 & not represented \\
\hline CLM5.0 & not represented \\
\hline CWatM & not represented \\
\hline DBH & not represented \\
\hline H08 & not represented \\
\hline JULES-W1 & not represented \\
\hline LPJmL & not represented \\
\hline Mac-PDM.20 & not represented \\
\hline MATSIRO & not represented \\
\hline $\mathrm{mHM}$ & not represented \\
\hline MPI-Hm & $Q_{w e}=\frac{S_{w e}}{t_{w e}}$ \\
\hline & for $t_{w e}=\frac{l_{G, r i}}{C_{M S, w e} \times h_{w, w e^{\frac{2}{3}}} \times s_{w e, m e a n}{ }^{\frac{1}{2}}}$ \\
\hline ORCHIDEE & not represented \\
\hline PCR-GLOBWB & not represented \\
\hline VIC & not represented \\
\hline WaterGAP2 & $Q_{w e, \text { local }}=C_{s w, \text { out }} \times S_{\text {we }, \text { local }} \times\left(\frac{S_{w e, l o c a l}}{S_{w e, \text { ocal } \max }}\right)^{2.5}$ \\
\hline WAYS & $\begin{array}{l}Q_{w e, g l o b a l}=C_{s w, \text { out }} \times S_{w e, \text { global }} \\
\text { with } C_{s w, \text { out }}=0.01 d^{-1} \\
\text { not represented }\end{array}$ \\
\hline
\end{tabular}


Table S43. River storage $S_{r i}$

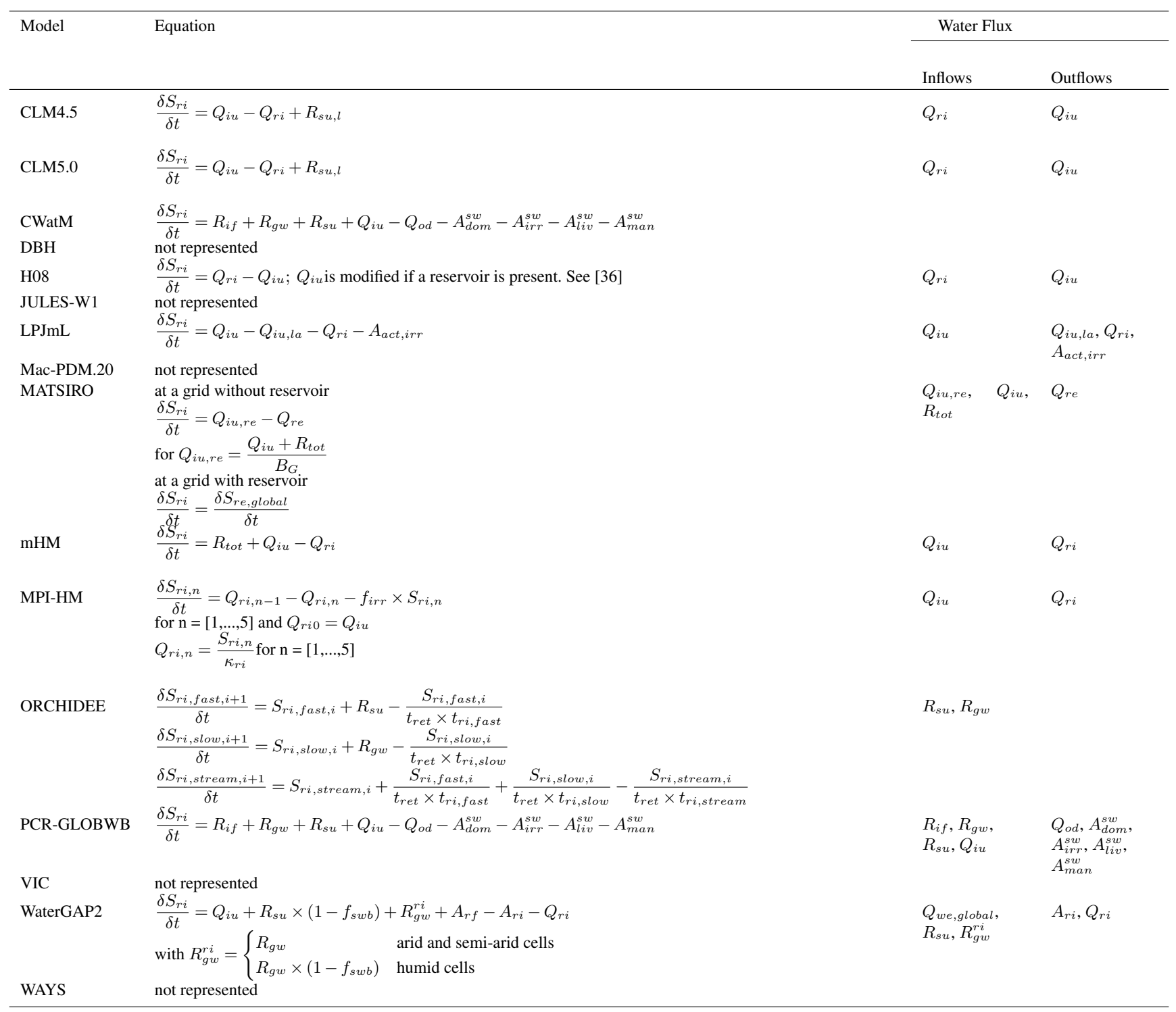


Table S44. Rivulet storage $S_{r v}$

\begin{tabular}{llcc}
\hline Model & Equation & Water Flux \\
\cline { 2 - 3 } & & Inflows & Outflows \\
\hline CLM4.5 & not represented & & \\
CLM5.0 & not represented & $R_{s u_{L C, R}}$ & $R_{G}^{1}$ \\
CWatM & $\frac{\delta S_{r v}}{\delta t}=R_{s u_{L C, R}}-R_{G}^{1}$ & \\
DBH & not represented & \\
H08 & not represented & \\
JULES-W1 & not represented & \\
LPJmL & not represented & \\
Mac-PDM.20 & not represented & \\
MATSIRO & not represented & \\
mHM & not represented & \\
MPI-HM & $\delta S_{r v}=R_{s u}-R_{G}$ & \\
ORCHIDEE & not represented & \\
PCR-GLOBWB & not represented & \\
VIC & not represented & \\
Water-GAP2 & not represented & \\
WAYS & not represented & \\
\hline
\end{tabular}


Table S45. Inflow from upstream surface water bodies $Q_{i u}$

\begin{tabular}{|c|c|}
\hline Model & Equation \\
\hline CLM4.5 & sum of inflows of water from neighboring upstream grid cells \\
\hline CLM5.0 & sum of inflows of water from neighboring upstream grid cells \\
\hline CWatM & sum of inflows of water from neighboring upstream grid cells, lakes and reservoirs. Kinematic wave approach. \\
\hline $\mathrm{DBH}$ & not represented \\
\hline $\mathrm{H} 08$ & $Q_{i u G}=v \times S_{r i G-1}$ for $v=0.5$ \\
\hline JULES-W1 & not represented \\
\hline LPJmL & $Q_{i u}=Q_{r i}-E_{l a}-E_{r e}$ \\
\hline Mac-PDM.20 & not represented \\
\hline MATSIRO & $Q_{i u}=\sum_{\text {upstreamG }} \times Q_{\text {ri }}{ }^{\text {upstream } G}$ \\
\hline mHM & sum of inflows of water from neighboring upstream grid cells \\
\hline MPI-HM & $Q_{i u}=R_{g w, u p}+Q_{r v, u p}+Q_{r i, u p}-Q_{i u, w e, u p}$ \\
\hline ORCHIDEE & $Q_{i u}=\sum \frac{S_{\text {ri,stream,upper }}}{t_{\text {ret }} \times t_{\text {ri,stream }}}$ \\
\hline PCR-GLOBWB & $Q_{i u}=\frac{\delta Q_{r i}}{\delta l_{r i}}+C_{w e i r} \times C_{w e i r} \times Q_{r i}{ }^{\left(C_{w e i r}-1\right)} \times \frac{\delta Q_{r i}}{\Delta t}$ \\
\hline VIC & not represented \\
\hline WaterGAP2 & inflow from upstream grid cells is routed through global lakes see Table S32. \\
\hline WAYS & not represented \\
\hline
\end{tabular}


Table S46. Streamflow $Q_{r i}$

\begin{tabular}{|c|c|}
\hline Model & Equation \\
\hline CLM4.5 & $Q_{r i}=\frac{v}{l_{G}} \times S_{r i}$ \\
\hline CLM5.0 & $Q_{r i}=\frac{C_{r i, h y d r a u l i c}^{\frac{2}{3}} \times s_{r i}}{C_{M}}$ \\
\hline CWatM & $\begin{array}{l}\frac{\Delta t}{l_{r i}} \times Q_{r i i+1}^{t+1}+\alpha \times\left(Q_{r i i+1}^{t+1}\right)^{\beta}=\frac{\Delta t}{l_{r i}} \times Q_{r i i}^{t+1}+\alpha \times\left(Q_{r i i+1}^{t}\right)^{\beta}+\Delta t \times\left(\frac{Q_{r i}^{t+1}+{ }_{i+1}+Q_{r i i+1}^{t}}{2}\right) \\
\text { For each cell and for each time step using an iterative approach given in Chow et al. [9]. The coefficients can } \\
\text { be calculated using Manning's equation. }\end{array}$ \\
\hline DBH & not represented \\
\hline H08 & $Q_{r i}=\sum R_{s u}+Q_{i u}$ \\
\hline JULES-W1 & $\begin{array}{l}\text { When a dam is present, outflow from dam is used, see Table } 35 \text {. } \\
\text { not represented }\end{array}$ \\
\hline LPJmL & $Q_{r i, \text { out }}=Q_{r i, \text { in }} \times \frac{1}{\iota \times \Gamma \times n} \times \frac{t}{\iota}^{(n-1)} \times e^{\frac{-t}{\iota}}$ \\
\hline Mac-PDM.20 & $\begin{array}{l}\text { for: } n=\frac{l_{r i}}{l_{r i, h o m}} ; \iota=\frac{l_{r i, h o m}}{v_{\text {mean }}} ; \Gamma=\text { gamma function which allows for non-integer values of "n". } \\
\text { not represented }\end{array}$ \\
\hline MATSIRO & $\begin{array}{l}\text { at a grid without reservoir } \\
Q_{r i, t}=\left(Q_{i u}+R_{t o t}{ }^{*}\right)-\frac{S_{r i,(t+\Delta t)}-S_{r i, t}}{\Delta t} \times B-\left(A_{i r r}^{r i}+A_{d o m}^{r i}+A_{i n d}^{r i}\right) \times B\end{array}$ \\
\hline $\mathrm{mHM}$ & $\begin{array}{l}\text { A part of } R_{t o t} \text { flows into a pond and is stored in the pond, then } R_{t o t} \text { becomes } R_{t o t}{ }^{*} \text { which }=Q_{r e, l o c a l} \\
\text { at a grid with reservoir } \\
Q_{r i}=Q_{r e}-\left(A_{i r r}^{r i}+A_{d o m}^{r i}+A_{i n d}^{r i}\right) \times B \\
Q_{r i} t+1=C_{M 1} \times Q_{r i i}{ }_{t}+1 \\
\text { for each cell } i \text { and for each time step } t \text { using an iterative approach given in Chow et al. [9]. The coefficients } \\
C_{M 1}-C_{M 3} \text { are fully derived in Thober et al. [50]. }\end{array}$ \\
\hline MPI-HM & $Q_{r i}=\frac{S_{r i, n}}{\kappa_{r i}}$ for $\mathrm{n}=5$ \\
\hline ORCHIDEE & $Q_{r i}=\frac{S_{\text {ri,stream }, i}}{t_{\text {ret }} \times t_{\text {ri,stream }}}$ \\
\hline $\begin{array}{l}\text { PCR-GLOBWB } \\
\text { VIC }\end{array}$ & $\begin{array}{l}Q_{r i}=\frac{\partial Q_{r i}}{\delta l_{r i}}+C_{w e i r} \times C_{w e i r} \times Q_{r i}{ }^{\left(C_{w e i r}-1\right)} \times \frac{\partial Q_{r i}}{\Delta t} \\
\text { not represented }\end{array}$ \\
\hline WaterGAP2 & $\begin{array}{l}\text { not represented } \\
Q_{r i}=\frac{v}{l_{r i}} \times S_{r i}\end{array}$ \\
\hline WAYS & $\begin{array}{l}\text { for } v=C_{M}{ }^{-1} \times C_{r i, h y d r a u l i c} \frac{2}{3} \times s_{r i}{ }^{\frac{1}{2}} \\
\text { for details see Verzano et al. [52] } \\
\text { not represented }\end{array}$ \\
\hline
\end{tabular}


Table S47. Evaporation from river $E_{r i}$

\begin{tabular}{ll}
\hline Model & Equation \\
\hline CLM4.5 & not represented \\
CLM5.0 & not represented \\
CWatM & $E_{r i}=E_{l a, p o t}-\min \left(P_{t o t}, E_{l a, p o t}\right)$ \\
DBH & not represented \\
H08 & not represented \\
JULES-W1 & not represented \\
LPJmL & $E_{r i}=E_{l a}=\min \left(S_{l a}, E_{l a, p o t}\right)$ \\
Mac-PDM.20 & not represented \\
MATSIRO & not represented \\
mHM & not represented \\
MPI-HM & not represented \\
ORCHIDEE & not represented \\
PCR-GLOBWB & $E_{r i}=P E T$ \\
VIC & not represented \\
WaterGAP2 & not represented \\
WAYS & not represented \\
\hline
\end{tabular}


Table S48. Minimum and Maximum values of each water storage Part I

\begin{tabular}{|c|c|c|c|c|c|c|c|c|}
\hline \multirow[t]{2}{*}{ Water Storage } & \multicolumn{2}{|l|}{ CLM4.5 } & \multicolumn{2}{|c|}{ CLM5.0 } & \multicolumn{2}{|l|}{ CWatM } & \multicolumn{2}{|l|}{$\mathrm{DBH}$} \\
\hline & Min & Max & Min & Max & Min & Max & Min & $\operatorname{Max}$ \\
\hline Canopy storage & 0 & $\begin{array}{l}\text { depends on } \\
\text { vegetation } \\
\text { characteristics } \\
\text { (>20 PFT) and } \\
\text { state (LAI) }\end{array}$ & 0 & $\begin{array}{l}\text { depends on } \\
\text { vegetation } \\
\text { characteristics } \\
\text { (>20 PFT) and } \\
\text { state (LAI) }\end{array}$ & 0 & $\begin{array}{l}\text { specific to each land } \\
\text { cover class and time } \\
\text { of the year }\end{array}$ & 0 & $\begin{array}{l}\left(2 \times 10^{-4} \text { and } 5 \times\right. \\
\left.10^{-4}\right) \times L A I\end{array}$ \\
\hline Snow storage & 0 & no upper limit & 0 & no upper limit & 0 & no upper limit & $\begin{cases}0, & \text { for canopy } \\
0.002 m, & \text { for ground } \\
& \text { surface }\end{cases}$ & $\begin{cases}10^{-4} \times & \text { for canopy } \\
L A I \times & \\
f_{c a, e x}, & \\
\text { no limit, } & \text { for ground } \\
& \text { surface }\end{cases}$ \\
\hline Soil storage & 0 & $100 \%$ & 0 & $100 \%$ & 0 & $\begin{array}{l}\text { water content - } \\
\text { residual soil layer } \\
\text { water content) x soil } \\
\text { layer depth }\end{array}$ & 0 & $\begin{array}{l}100 \%, \text { absolute } \\
\text { capacity is } \\
\text { determined by soil } \\
\text { properties }\end{array}$ \\
\hline Groundwater storage & 0 & $\begin{array}{l}\text { no upper limit } \\
\text { the storage is } \\
\text { forced constant } \\
\text { (any imbalance } \\
\text { between P and } \\
\text { ET is }\end{array}$ & 0 & $\begin{array}{l}4800 \mathrm{~mm} \\
\text { the storage is } \\
\text { forced constant } \\
\text { (any imbalance } \\
\text { between } P \text { and } \\
\text { ET is }\end{array}$ & 0 & no upper limit & not represented & not represented \\
\hline Lake storage & 0 & $\begin{array}{l}\text { compensated } \\
\text { by an artificial } \\
\text { runoff term, } \\
\text { keeping lake } \\
\text { depth at a } \\
\text { constant value) }\end{array}$ & 0 & $\begin{array}{l}\text { compensated } \\
\text { by an artificial } \\
\text { runoff term, } \\
\text { keeping lake } \\
\text { depth at a } \\
\text { constant value) }\end{array}$ & 0 & no upper limit & not represented & not represented \\
\hline Wetland storage & not represented & not represented & not represented & not represented & not represented & not represented & not represented & not represented \\
\hline Reservoir storage & not represented & not represented & not represented & not represented & 0 & $\begin{array}{l}\text { defined for each } \\
\text { reservoir, cannot be } \\
\text { exceed because of } \\
\text { included non } \\
\text { damaging outflow } \\
\text { function }\end{array}$ & 0 & $\begin{array}{l}\text { Reservoir storage } \\
\text { capacity }\end{array}$ \\
\hline River storage & not represented & not represented & not represented & not represented & 0 & no upper limit & 0 & $\infty$ \\
\hline
\end{tabular}


Table S49. Minimum and Maximum values of each water storage Part II

\begin{tabular}{|c|c|c|c|c|c|c|c|}
\hline \multirow[t]{2}{*}{ Water Storage } & \multicolumn{2}{|l|}{ H08 } & \multicolumn{2}{|l|}{ JULES-W1 } & \multicolumn{2}{|l|}{ LPJmL } & \multirow[t]{2}{*}{ MATSIRO } \\
\hline & Min & Max & Min & Max & Min & Max & \\
\hline Canopy storage & not represented & not represented & $S_{c a, \min }=0.5$ & $\begin{array}{l}S_{c a, \max }= \\
S_{c a, \min }+0.05 \times \\
L A I \\
\text { Minimum LAI } \\
\text { permitted in } \\
\text { calculation of the } \\
\text { albedo in snow-free } \\
\text { conditions is set to } \\
0.5 \mathrm{~m} 2 / \mathrm{m} 2, \\
\text { maximum LAI value } \\
\text { ranges between } 1 \\
\text { and } 5 \text { depending on } \\
\text { the plant functional } \\
\text { type (PFT). [5] }\end{array}$ & 0 & $\begin{array}{l}S_{c a, p} \text { specific to } \\
\text { each } P F T, \\
\text { respectively, tropical } \\
\text { and temperate trees } \\
0.02, \text { boreal trees } \\
0.06 \text {, grasses } 0.01 . \\
\text { Canopy water } \\
\text { storage is assumed to } \\
\text { be the product of } \\
\text { daily precipita2on, } \\
\text { leaf area index, and a } \\
\text { PFTand CFT-specific } \\
\text { parameter that } \\
\text { approximates the } \\
\text { leaf form of the } \\
\text { PFTs and the } \\
\text { precipita2on regime } \\
\text { (rainfall intensity) } \\
\text { where they typically } \\
\text { grow. The parameter } \\
\text { is tabulated in Gerten } \\
\text { et al. (2004). }\end{array}$ & not represented \\
\hline Snow storage & 0 & no upper limit & 0 & no upper limit & $0 \mathrm{~mm}$ & $20000 \mathrm{~mm}$ & not represented \\
\hline Soil storage & wilting point & field capacity & $\begin{array}{l}\text { Depends on the soil } \\
\text { type per grid cell. } \\
\text { Defined per grid cell } \\
\text { ( } 0.5 \text { degrees). } \\
\text { Volumetric soil } \\
\text { moisture content at } \\
\text { the wilting point (m3 } \\
\text { water per } 3 \text { soil) } \\
\text { i.e. the point at } \\
\text { which soil moisture } \\
\text { stress completely } \\
\text { prevents } \\
\text { transpiration, ranges } \\
\text { between } 0 \text { (for ice } \\
\text { covered regions) and } \\
0.263 \text {. The } \\
\text { configuration uses } \\
\text { soil data from the } \\
\text { Harmonized World } \\
\text { Soil Database }\end{array}$ & $\begin{array}{l}\text { Depends on the soil } \\
\text { type per grid cell. } \\
\text { Defined per grid cell } \\
\text { ( } 0.5 \text { degrees). The } \\
\text { volumetric soil } \\
\text { moisture content at } \\
\text { saturation (m3 water } \\
\text { per m3 soil) ranges } \\
\text { between } 0 \text { (for ice } \\
\text { covered regions) and } \\
0.458 \text {. The } \\
\text { configuration uses } \\
\text { soil data from the } \\
\text { Harmonized World } \\
\text { Soil Database. [35] }\end{array}$ & 0 & $\begin{array}{l}S_{s o, s a t, p} \text { specific to } \\
\text { soil type clay }=0.468 ; \\
\text { silty clay }=0.468 ; \text { sandy } \\
\text { clay }=0.406 ; \text { clay } \\
\text { loam }=0.465 ; \text { silty clay } \\
\text { loam }=0.464 ; \text { sandy } \\
\text { clay loam }=0.404 ; \\
\text { loam }=0.439 ; \text { silt } \\
\text { loam }=0.476 ; \text { sandy } \\
\text { loam }=0.434 ; \\
\text { silt }=0.476 ; \text { loamy } \\
\text { sand }=0.421 ; \\
\text { sand }=0.339 ; \text { rock and } \\
\text { ice }=0.006 .\end{array}$ & $\begin{array}{l}\text { Soil storage is } \\
\text { constrained by soil } \\
\text { layer thickness and } \\
\text { porosity }\end{array}$ \\
\hline Groundwater storage & 0 & no upper limit & not represented & not represented & not represented & not represented & not represented \\
\hline Lake storage & not represented & not represented & not represented & not represented & 0 & $f_{l a} \times 5 \times G \times 1000$ & not represented \\
\hline Wetland storage & not represented & not represented & not represented & not represented & not represented & not represented & not represented \\
\hline Reservoir storage & 0 & dam capacity & not represented & not represented & 0 & $\begin{array}{l}\text { Reservoir dependent, } \\
\text { this is an input to the } \\
\text { model }\end{array}$ & $\begin{array}{l}\text { Reservoir storage } \\
\text { cannot exceed } \\
\text { storage capacity } \\
\text { specified in GRanD } \\
\text { data }\end{array}$ \\
\hline River storage & no limit & no limit & not represented & not represented & 0 & no upper limit & not represented \\
\hline
\end{tabular}


Table S50. Minimum and Maximum values of each water storage Part III

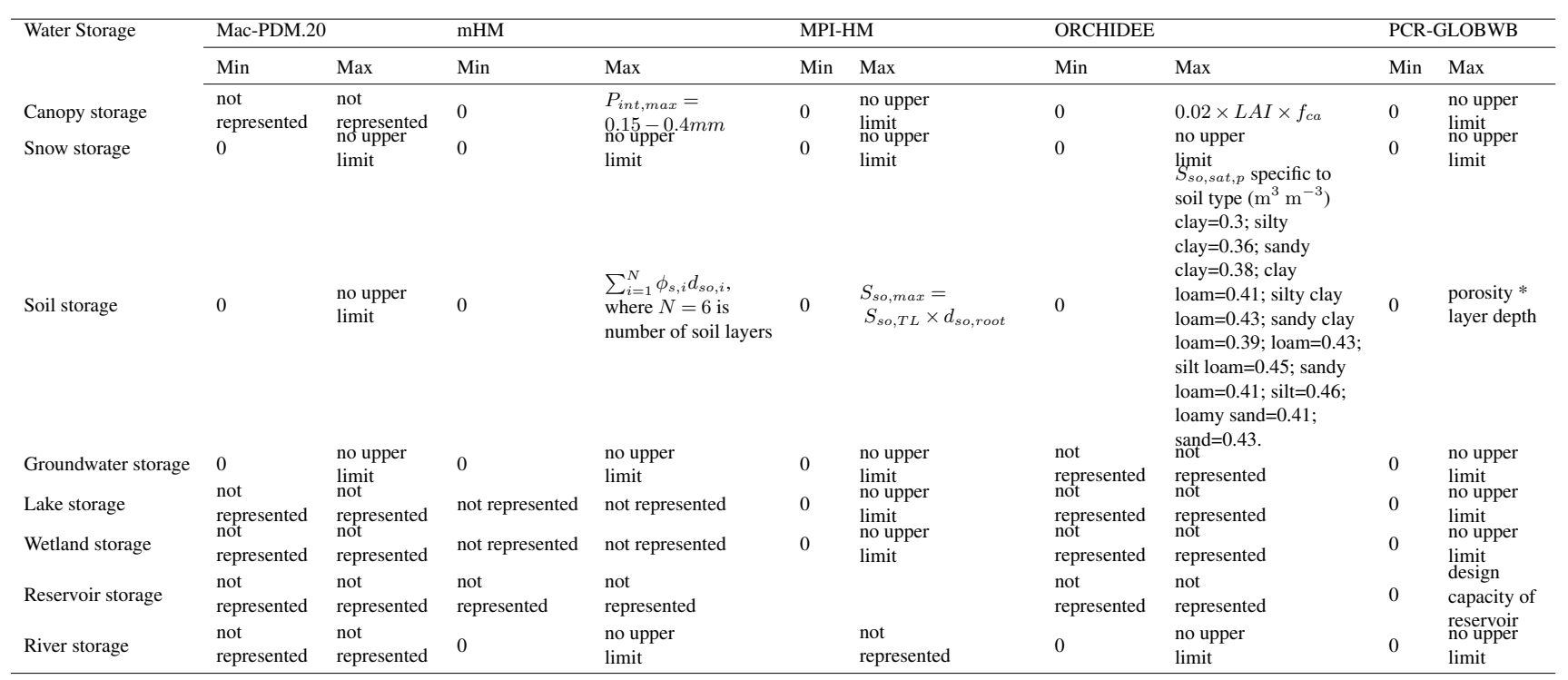


Table S51. Minimum and Maximum values of each water storage Part IV

\begin{tabular}{|c|c|c|c|c|c|c|}
\hline \multirow[t]{2}{*}{ Water Storage } & \multicolumn{2}{|r|}{ VIC } & \multicolumn{2}{|c|}{ WaterGAP2 } & \multicolumn{2}{|r|}{ WAYS } \\
\hline & Min & Max & Min & Max & Min & Max \\
\hline Canopy storage & 0 & no upper limit & 0 & $S_{c a, \max }=0.3 \times L A I$ & 0 & $\mathrm{f}\left(L A I_{\max }\right)$ \\
\hline Snow storage & 0 & no upper limit & 0 & $1000 \mathrm{~mm}$ & 0 & no upper limit \\
\hline Soil storage & 0 & porosity * layer depth & 0 & $S_{s o, \max }$ & 0 & $S_{r z, \max }$ \\
\hline Groundwater storage & 0 & no value & no limit & no limit & 0 & no upper limit \\
\hline Lake storage & 0 & no upper limit & $\begin{array}{l}-S_{l a, l o c a l, \max } \\
-S_{l a, g l o b a l, \max }\end{array}$ & $\begin{array}{l}S_{l a, \text { local }, \max }= \\
B_{l a, \text { local }, \text { max }} \times 5 \mathrm{~m} \\
S_{l a, \text { global }, \text { max }}= \\
B_{\text {la,global } \text { max }} \times 5 \mathrm{~m}\end{array}$ & & not represented \\
\hline Wetland storage & 0 & no upper limit & 0 & $\begin{array}{l}S_{w e, l o c a l, \text { max }}= \\
B_{w e, \text { local }, \text { max }} \times 2 m \\
S_{w e, \text { global }, \text { max }}= \\
B_{w e, \text { global }, \text { max }} \times 2 m\end{array}$ & & not represented \\
\hline Reservoir storage & 0 & design capacity of reservoir & 0 & $S_{r e, C} \times 0.85$ & & not represented \\
\hline River storage & 0 & no value & 0 & no limit & 0 & no upper limit \\
\hline
\end{tabular}


Table S52. Irrigation water demand $A_{i r r}$

\begin{tabular}{|c|c|}
\hline Model & Equation \\
\hline CLM4.5 & not represented \\
\hline CLM5.0 & $A_{i r r}=j \times S_{s o, s a t}+(1-j) \times S_{s o, W P}-S_{s o, c u r}$ \\
\hline CWatM & not represented \\
\hline DBH & not represented \\
\hline H08 & not represented \\
\hline JULES-W1 & not represented \\
\hline LPJmL & not represented \\
\hline Mac-PDM.20 & not represented \\
\hline MATSIRO & $A_{i r r}=\frac{\rho_{w}}{\Delta t} \times \sum_{i=1}^{3} \max \left[\left(\phi_{t, i}-\phi_{a, i}\right), 0\right] \times \zeta_{s o, i}$ \\
\hline $\mathrm{mHM}$ & $\begin{array}{l}\phi_{t, i}=\alpha \times \phi_{\text {so }}{ }^{i=1} \\
\alpha \text { is set at } 1 \text { for rice and } 0.75 \text { for the other crops, see [38]. } \\
\text { not represented }\end{array}$ \\
\hline MPI-HM & $A_{i r r}=\left(T \times \frac{f_{c a}-f_{i r r}}{f_{c a}}+P E T \times f_{i r r}\right)-T$ \\
\hline & $f_{i r r}=1-\frac{A_{i r r}}{\sum S_{i}} ;$ for $\mathrm{i}=$ baseflow, river, wetland \\
\hline ORCHIDEE & not represented \\
\hline PCR-GLOBWB & not represented \\
\hline VIC & not represented \\
\hline WaterGAP2 & not represented \\
\hline WAYS & not represented \\
\hline
\end{tabular}


Table S53. Irrigation groundwater abstraction $A_{i r r}^{g w}$

\begin{tabular}{|c|c|}
\hline Model & Equation \\
\hline CLM4.5 & not represented \\
\hline CLM5.0 & not represented \\
\hline CWatM & $A_{\text {pot }}^{g w}=A_{\text {tot }}-A_{\text {act }}^{s w}$ \\
\hline & $A_{a c t}^{g w}=\min \left(S_{g w}-A_{p o t}^{g w}\right)$ \\
\hline & $A_{f g w}=A_{\text {pot }}^{g w}-A_{\text {act }}^{g w}$ \\
\hline DBH & not represented \\
\hline $\mathrm{H} 08$ & 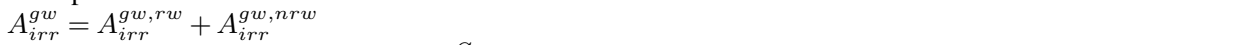 \\
\hline & $A_{i r r}^{g w, r w}=\min \left(f_{g w, u s e} \times A_{i r r, d e m} \times \frac{S_{g w, r w}}{\Delta t}\right)$ \\
\hline JULES-W1 & $A_{i r r}^{g w, n r w}=f_{g w, u s e} \times A_{i r r, d e m}-A_{g w, r w} \Delta t$ \\
\hline LPJmL & not represented \\
\hline Mac-PDM.20 & not represented \\
\hline MATSIRO & $\begin{array}{l}\text { Given the amount of water requirement for irrigation, water is firstly taken from local river flow (potentially } \\
\text { regulated by global reservoir), then from local reservoir (same cell or upstream cell). Then, rest of water require- } \\
\text { ment unmet is taken from groundwater resource. Here MATSIRO assumes unlimited groundwater resource. } \\
A^{g w}=A_{\text {- }}\end{array}$ \\
\hline mHM & not represented \\
\hline MPI-HM & $A_{i r r}^{g w}=f_{i r r} \times S_{g w}$ \\
\hline ORCHIDEE & not represented \\
\hline PCR-GLOBWB & not represented \\
\hline VIC & not represented \\
\hline WaterGAP2 & $A_{\text {irr }}^{g w}=\frac{A_{\text {irr }, \text { cons }}}{07} \times f_{\text {gw }, \text { use }}$ \\
\hline WAYS & $\begin{array}{l}A_{i r r, c o n s} \text { is calculated with a Global Irrigation Model, see [34] and [12]. } \\
\text { not represented }\end{array}$ \\
\hline
\end{tabular}


Table S54. Irrigation groundwater consumption $A_{i r r, c o n s}^{g w}$

\begin{tabular}{ll}
\hline Model & Equation \\
\hline CLM4.5 & not represented \\
CLM5.0 & not represented \\
CWatM & $A_{g w, \text { cons }}=A_{t o t, c o n s}-A_{s w, \text { cons }}$ \\
DBH & not represented \\
H08 & $A_{i r r, \text { cons }}^{g w}=f_{\text {cons }, A} \times A_{i r r}^{g w}$ \\
JULES-W1 & not represented \\
LPJmL & not represented \\
Mac-PDM.20 & not represented \\
MATSIRO & Theoretically speaking, $A_{i r r, c o n s}^{g w}=X \times A_{i r r}^{g w}$ where $X$ is overall irrigation efficiency (which does not consider \\
mHM & combinations of irrigation system type and water sources.) \\
MPI-HM & not represented \\
ORCHIDEE & no water losses are computed \\
PCR-GLOBWB & not represented \\
VIC & $A_{i r r, c o n s}^{g w}=\frac{R_{g w}}{R_{g w}+Q_{i u}} \times A_{i r r} \times X$ \\
WaterGAP2 & not represented \\
WAYS & $A_{i r r, c o n s}^{g w}=A_{i r r, c o n s} \times f_{g w, u s e} A_{i r r, c o n s}$ is calculated with a Global Irrigation Model, see [34] and [12]. \\
\hline
\end{tabular}


Table S55. Return flow from irrigation groundwater abstraction $A_{i r r, r f}^{g w}$

\begin{tabular}{ll}
\hline Model & Equation \\
\hline CLM4.5 & not represented \\
CLM5.0 & not represented \\
CWatM & not represented \\
DBH & not represented \\
H08 & $A_{i r r, r f}^{g w}=\left(1-f_{\text {lost }}\right) \times\left(1-f_{\text {cons }, A}\right) \times A_{i r r}^{g w}[24]$ \\
JULES-W1 & not represented \\
LPJmL & not represented \\
Mac-PDM.20 & not represented \\
MATSIRO & In MATSIRO, return flow is implicitly accounted for. \\
& MATSIRO estimates potential irrigation water amount to keep soil moisture at the target level of 0.75. Irrigation \\
& water added, added as sprinkler irrigation, can percolate into deeper layers, ultimately recharging groundwater, \\
mHM & or contribute to local runoff depending on the rate of consumptive use by crops and soil wetness conditions. \\
MPI-HM & not represented \\
ORCHIDEE & not represented \\
PCR-GLOBWB & not represented \\
VIC & $A_{i r r, r f}^{g w}=\frac{R_{g w}}{R_{g w}+Q_{i u}} \times A_{i r r} \times(1-X)$ \\
WaterGAP2 & not represented \\
WAYS & $A_{i r r, r f}^{g w}=A_{i r r}^{g w}-A_{i r r, c o n s}^{g w}$ \\
\hline
\end{tabular}


Table S56. Irrigation surface water abstraction $A_{i r r}^{s w}$

\begin{tabular}{|c|c|}
\hline Model & Equation \\
\hline CLM4.5 & $A_{i r r}^{s w}=\sum \max \left(\phi_{t, i}-\phi_{a, i}, 0\right)$ \\
\hline CLM5.0 & $\begin{array}{l}\text { with: } \phi_{t, i}=(1-0.7) \times \phi_{m i n, i}+0.7 \times \phi_{s, i} \\
A_{i r r}^{s w}=A_{i r r}\end{array}$ \\
\hline \multirow[t]{2}{*}{ CWatM } & $A_{i r r}^{s w}=\frac{A_{i r r, \text { cons }}}{f_{\text {irr, eff }}}$ \\
\hline & $\begin{array}{l}f_{\text {irr }, \text { eff }} \text { : see [46]. } \\
\text { It also simulates overflow of the flooded topsoil for paddy rice land use. This saturation excess it is not controlled } \\
\text { by soil hydraulic properties, but by human intervention. } \\
R_{o f}=\max \left(0, S_{\text {paddy }}-0.05 \mathrm{~m}\right) ; S_{\text {paddy }}=\text { the storage of flooded paddy rice (only for paddy rice land use) }\end{array}$ \\
\hline DBH & not represented \\
\hline H08 & $A_{i r r}^{s w}=A_{i r r}^{a q}+A_{i r r}^{r e}+A_{i r r}^{r i}+A_{i r r}^{u s w}$ \\
\hline JULES-W1 & not represented \\
\hline LPJmL & $A_{i r r}^{s w}=A_{i r r}^{l a}+A_{i r r}^{n e i g h, c e l l}+A_{i r r}^{r e}+A_{i r r}^{r i}$ \\
\hline Mac-PDM.20 & not represented \\
\hline MATSIRO & $\begin{array}{l}\text { Given the amount of water requirement for this sector, water is firstly taken from local river flow (potentially } \\
\text { regulated by global reservoir), then from local reservoir (same cell or upstream cell). } \\
A_{i r r}^{s w}=A_{i r r}^{\text {pond }}+A_{i r r}^{r e, g l o b a l}+A_{i r r}^{r i}\end{array}$ \\
\hline $\mathrm{mHM}$ & not represented \\
\hline MPI-HM & $A_{i r r}^{s w}=\sum_{n=1}\left(f_{i r r} \times S_{r i, n}\right)+f_{i r r} \times S_{w e}$ \\
\hline ORCHIDEE & not represented \\
\hline PCR-GLOBWB & $A_{i r r}^{s w}=\frac{Q_{i u}}{R_{g w}+Q_{i u}} \times A_{i r r}$ \\
\hline VIC & not represented \\
\hline WaterGAP2 & $A_{i r r}^{s w}=\frac{A_{i r r, c o n s}}{f_{i r r, s w, e f f}} \times\left(1-f_{g w, u s e}\right) A_{i r r, c o n s}$ is calculated with a Global Irrigation Model, see [34] and [12]. \\
\hline WAYS & $\begin{array}{l}J i r r, s w, e f f \\
\text { not represented }\end{array}$ \\
\hline
\end{tabular}


Table S57. Irrigation surface water consumption $A_{i r r, \text { cons }}^{s w}$

\begin{tabular}{|c|c|}
\hline Model & Equation \\
\hline CLM4.5 & not represented \\
\hline CLM5.0 & $A_{i r r, c o n s}^{s w}=A_{i r r}^{s w}-A_{i r r, r f}^{s w}$ \\
\hline \multirow[t]{9}{*}{ CWatM } & For paddy irrigation: \\
\hline & $\begin{array}{l}A_{\text {irr,cons }}=\left(d_{\text {paddy, } \max }^{\text {sw }}-\left(d_{\text {paddy }}^{\text {sw }}{ }^{t-1}-P_{\text {eff }}{ }^{t}\right)\right) \\
d_{\text {paddy }}^{\text {sw }}{ }^{t}=d_{\text {paddy }}^{\text {sw }}{ }^{t-1}+P_{\text {eff }}{ }^{t}+A_{\text {irr }}+R_{\text {in }}-E_{\text {osw }}{ }^{d, t} \\
\text { For non paddy: }\end{array}$ \\
\hline & $\quad \int S_{s o, T 2 L}-S_{\text {so, ready }} \quad\left(S_{\text {so,ready }}<S_{\text {so }, \text { crit }}\right)$ \\
\hline & $A_{\text {irr }, \text { cons }}= \begin{cases}0 & \left(S_{\text {so }, \text { ready }} \geq S_{\text {so }, \text { crit }}\right)\end{cases}$ \\
\hline & $S_{s o, T 2 L}=S_{s o, F C}-S_{s o, W P}$ \\
\hline & $S_{\text {so }, \text { ready }}=S_{\text {so }}-S_{\text {so }, W P}$ \\
\hline & $S_{\text {so }, \text { crit }}=(1-p) \times S_{s o, T 2 L}+S_{s o, W P}$ \\
\hline & $p=\frac{1}{1}-0.4+\frac{(P E T-0.6)}{\text { see }[51] .}$ \\
\hline & $p=\overline{(0.76+1.5 \times P E T)}-0.4+\frac{4}{4}$ \\
\hline $\begin{array}{l}\mathrm{DBH} \\
\mathrm{HOS}\end{array}$ & $\begin{array}{l}\text { not represented } \\
A^{s w}-A^{s w}\end{array} A^{s w} \quad+A^{s w}$ \\
\hline & $\begin{array}{c}A_{i r r}^{s w}=A_{i r r, c o n s}^{s w}+A_{i r r, l o s s}^{s w}+A_{i r r, r f}^{s w} \\
A^{s w}=A_{i w}^{s w}\end{array}$ \\
\hline & $\begin{array}{l}A_{i r r, \text { cons }}=f_{\text {cons }, A} \times A_{\text {irr }}^{\text {iv }} \\
A_{\text {irr }, \text { loss }}^{s w}=f_{\text {lost }} \times\left(1-f_{\text {cons }, A}\right) \times A_{\text {irr }}^{s w}\end{array}$ \\
\hline JULES-W1 & not represented \\
\hline LPJmL & $A_{\text {irr }, \text { net }}=\max \left(0, S_{\text {so }, F C}-S_{\text {so, ready }}\right)$ \\
\hline & $A_{\text {irr }, \text { gross }, G}=\frac{A_{i r r, n e t}+A_{i r r, a p p}-S_{b u f}}{X}, X_{c o n v}=0.95$, see [7] \\
\hline Mac-PDM.20 & $\begin{array}{l}A_{i r r, a p p}=\max \left(0,\left(S_{s o, s a t}-X_{\text {conv }, F C}\right) \times \lambda-S_{\text {so,ready }}\right), \text { see [41] } \\
\text { not represented }\end{array}$ \\
\hline MATSIRO & Theoretically speaking, $A_{i r r, c o n s}^{s w}=X \times A_{i r r}^{s w}$ \\
\hline mHM & not represented \\
\hline MPI-HM & not represented \\
\hline ORCHIDEE & not represented \\
\hline PCR-GLOBWB & $A_{i r r, c o n s}^{s w}=\frac{Q_{i u}}{R_{\text {no }}+Q_{i r}} \times A_{i r r}$ \\
\hline VIC & not represented \\
\hline WaterGAP2 & $A_{i r r, \text { cons }}^{s w}=A_{i r r, \text { cons }} \times\left(1-f_{g w, u s e}\right)$ \\
\hline WAYS & $\begin{array}{l}A_{i r r, c o n s} \text { is calculated with a Global Irrigation Model, see [34] and [12]. } \\
\text { not represented }\end{array}$ \\
\hline
\end{tabular}


Table S58. Return flow from irrigation surface water abstraction $A_{i r r, r f}^{s w}$

\begin{tabular}{|c|c|}
\hline Model & Equation \\
\hline CLM4.5 & not represented \\
\hline CLM5.0 & $A_{i r r, r f}^{s w}=f_{G, s a t} \times A_{i r r}^{s w}$ \\
\hline CWatM & $A_{i r r, r f}^{s w}=f_{i r r, e f f} \times A_{i r r}, \operatorname{see}[12]$ \\
\hline DBH & not represented \\
\hline H08 & $A_{i r r, r f}^{s w}=\left(1-f_{\text {lost }}\right) \times\left(1-f_{\text {cons }, A}\right) \times A_{i r r}^{s w}$. See [12], [24] \\
\hline JULES-W1 & not represented \\
\hline LPJmL & $A_{i r r, r f}^{s w}=A_{i r r, g r o s s, G}-A_{i r r, n e t}-A_{i r r, a p p}-E_{o s w}$ \\
\hline Mac-PDM.20 & $\begin{array}{l}\text { Where: } E_{o s w}=\left(A_{\text {irr }, \text { gross }, G}-A_{\text {irr }, \text { net }}-A_{\text {irr }, \text { app }}\right) \times 0.5 \\
\text { not represented }\end{array}$ \\
\hline MATSIRO & $\begin{array}{l}\text { Return flow from irrigation using surface water is not separately estimated in MATSIRO; this component is a } \\
\text { part of the return flow from the total water use within a grid cell. }\end{array}$ \\
\hline mHM & not represented \\
\hline MPI-HM & not represented \\
\hline ORCHIDEE & not represented \\
\hline PCR-GLOBWB & not represented \\
\hline VIC & not represented \\
\hline WaterGAP2 & $A_{i r r, r f}^{s w}=A_{i r r}^{s w}-A_{i r r, c o n s}^{s w}$ \\
\hline WAYS & not represented \\
\hline
\end{tabular}


Table S59. Equations for domestic groundwater abstraction $A_{d o m}^{g w}$

\begin{tabular}{ll}
\hline Model & Equation \\
\hline CLM4.5 & not represented \\
CLM5.0 & not represented \\
CWatM & not represented \\
DBH & not represented \\
H08 & $A_{d o m}^{g w}=A_{d o m}^{g w, r w}+A_{d o m}^{g w, n r w}$ \\
& $A_{d o m}^{g w, r w}=m i n\left(f_{g w, u s e} \times A_{d o m, d e m} \times \frac{S_{g w, r w}}{\Delta t}\right)$ \\
& $A_{d o m}^{g w, n r w}=f_{g w, u s e} \times A_{d o m, d e m}-A_{d o m}^{g w, r w}$ \\
JULES-W1 & not represented \\
LPJmL & prescribed data offered by ISIMIP2b framework \\
Mac-PDM.20 & not represented \\
MATSIRO & Given the amount of water requirement from the domestic sector, water is firstly taken from local river flow \\
& (potentially regulated by global reservoir), then from local reservoir (same cell or upstream cell). Then, rest of \\
& water requirement unmet is taken from groundwater resource. Here, MATSIRO assumes unlimited groundwater \\
mHM & resource. $A_{d o m}^{g w}=A_{d o m, d e m}-A_{d o m}^{s w}$ \\
MPI-HM & where $A_{d o m, d e m}$ is domestic sectoral water requirement. \\
ORCHIDEE & not represented \\
PCR-GLOBWB & not represented \\
VIC & not represented \\
WaterGAP2 & $A_{d o m}^{g w}=\frac{R_{g w}}{R_{g w}+Q_{i u}} \times A_{d o m, d e m}$ \\
& not represented \\
WAYS & $A_{d o m}^{g w}=A_{d o m} \times f_{g w, u s e}$ \\
& $A_{d o m}=C_{d o m}^{w u, i n t s} \times P O P$ \\
for $C_{d o m}^{w u}$, ints & see $[18]$. \\
& not represented
\end{tabular}


Table S60. Equations for domestic groundwater consumption $A_{d o m, c o n s}^{g w}$

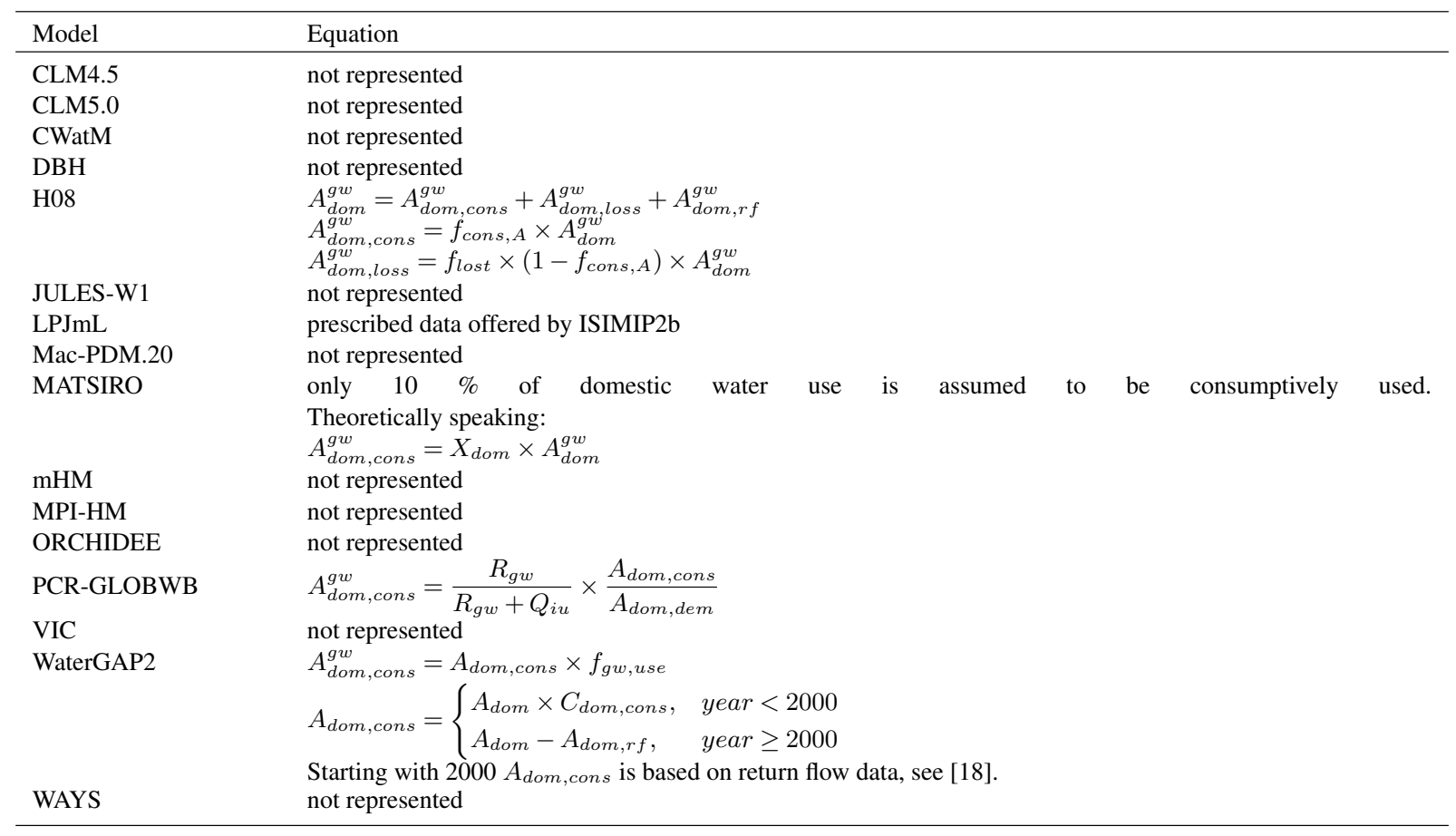


Table S61. Equations for return flow from domestic groundwater abstraction $A_{d o m, r f}^{g w}$

\begin{tabular}{|c|c|}
\hline Model & Equation \\
\hline CLM4.5 & not represented \\
\hline CLM5.0 & not represented \\
\hline CWatM & not represented \\
\hline DBH & not represented \\
\hline H08 & $A_{\text {dom }, r f}^{g w}=\left(1-f_{\text {lost }}\right) \times\left(1-f_{\text {cons }, A}\right) \times A_{\text {dom }}^{g w}$, see [24] \\
\hline JULES-W1 & not represented \\
\hline LPJmL & not represented \\
\hline Mac-PDM.20 & not represented \\
\hline MATSIRO & $\begin{array}{l}90 \% \text { of domestic water use is implicitly assumed to have returned to the original source (groundwater; see } \\
{[39] \text { ). }}\end{array}$ \\
\hline $\mathrm{mHM}$ & not represented \\
\hline MPI-HM & not represented \\
\hline ORCHIDEE & not represented \\
\hline PCR-GLOBWB & $A_{d o m, r f}^{g w}=\frac{R_{g w}}{R_{g w}+Q_{i y}} \times \frac{A_{d o m, d e m}-A_{d o m, c o n s}}{A_{d o m}}$ \\
\hline VIC & not represented \\
\hline WaterGAP2 & $A_{d o m, r f}^{g w}=A_{d o m, r f} \times f_{g w, \text { use }}$ \\
\hline & $A_{\text {dom }, r f}=\left\{\begin{array}{l}A_{\text {dom }}-\left(A_{\text {dom }} \times C_{\text {dom }, \text { cons }}\right) \quad \text { year }<2000 \\
\text { ( }\end{array}\right.$ \\
\hline WAYS & $\begin{array}{l}\quad A_{d o m, r f} \quad \text { year } \geq 2000 \\
\text { Starting with } 2000 A_{d o m, r f} \text { is based on wastewater volume data, see [18]. } \\
\text { not represented }\end{array}$ \\
\hline
\end{tabular}


Table S62. Equations for domestic surface water abstraction $A_{d o m}^{s w}$

\begin{tabular}{|c|c|}
\hline Model & Equation \\
\hline CLM4.5 & not represented \\
\hline CLM5.0 & not represented \\
\hline CWatM & not represented \\
\hline DBH & not represented \\
\hline H08 & $A_{d o m}^{s w}=A_{d o m}^{a q}+A_{d o m}^{r e}+A_{d o m}^{r i}+A_{d o m}^{u s w}$ \\
\hline JULES-W1 & not represented \\
\hline LPJmL & prescribed data offered by ISIMIP2b \\
\hline Mac-PDM.20 & not represented \\
\hline MATSIRO & $\begin{array}{l}\text { Given the amount of water requirement for this sector, water is firstly taken from local river flow (potentially } \\
\text { regulated by global reservoir), then from local reservoir (same cell or upstream cell). } \\
A_{d o m}^{s w}=A_{d o m}^{\text {pond }}+A_{d o m}^{r e, g l o b a l}+A_{d o m}^{r i}\end{array}$ \\
\hline mHM & not represented \\
\hline MPI-HM & not represented \\
\hline ORCHIDEE & not represented \\
\hline PCR-GLOBWB & $A_{d o m}^{s w}=\frac{Q_{i u}}{R_{g w}+Q_{i u}} \times A_{d o m, d e m}$ \\
\hline VIC & not represented \\
\hline WaterGAP2 & $\begin{array}{l}A_{d o m}^{\text {sw }}=A_{\text {dom }} \times\left(1-f_{g w, \text { use }}\right) \\
A_{\text {dom }}=C_{d o, \text { ints }}^{\text {wu }} \times P O P \\
\text { for } C_{\text {dom }}^{w u} \text {,ints } \text { see [18]. }\end{array}$ \\
\hline WAYS & not represented \\
\hline
\end{tabular}


Table S63. Equations for domestic surface water consumption $A_{d o m, c o n s}^{s w}$

\begin{tabular}{|c|c|}
\hline Model & Equation \\
\hline CLM4.5 & not represented \\
\hline CLM5.0 & not represented \\
\hline CWatM & not represented \\
\hline DBH & not represented \\
\hline H08 & 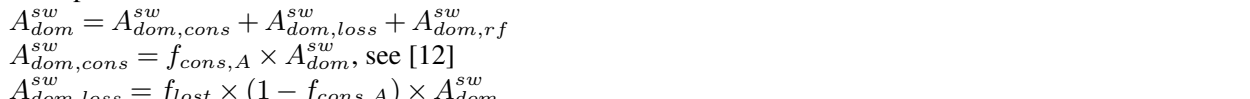 \\
\hline JULES-W1 & $\begin{array}{l}A_{\text {dom }, l o s s}=f_{\text {lost }} \times\left(1-f_{\text {cons }, A}\right) \times A_{\text {dom }} \\
\text { not represented }\end{array}$ \\
\hline LPJmL & prescribed data offered by ISIMIP2b \\
\hline Mac-PDM.20 & not represented \\
\hline MATSIRO & $\begin{array}{l}\text { only } 10 \% \text { of domestic water use, from surface water bodies, is assumed to be consumptively used. Theoretically } \\
\text { speaking: } A_{d o m, c o n s}^{s w}=X_{d o m} \times A_{d o m}^{s w}\end{array}$ \\
\hline $\mathrm{mHM}$ & not represented \\
\hline MPI-HM & not represented \\
\hline ORCHIDEE & not represented \\
\hline PCR-GLOBWB & $A_{d o m, \text { cons }}^{s w}=\frac{Q_{i u}}{R_{g w}+Q_{i u}} \times \frac{A_{d o m, c o n s}}{A_{d o m, d e m}}$ \\
\hline VIC & not represented \\
\hline WaterGAP2 & $A_{\text {dom }, \text { cons }}^{\text {sw }}=A_{\text {dom }, \text { cons }} \times\left(1-f_{g w, \text { use }}\right)$ \\
\hline & for $A_{d o m, c o n s}= \begin{cases}A_{d o m} \times C_{d o m, c o n s}, & \text { year }<2000 \\
A_{d o m}-A_{d o m, r f}, & \text { year } \geq 2000\end{cases}$ \\
\hline WAYS & $\begin{array}{l}\text { Starting with } 2000 A_{d o m, c o n s} \text { is based on return flow data, for details see [18]. } \\
\text { not represented }\end{array}$ \\
\hline
\end{tabular}


Table S64. Equations for return flow from domestic surface water abstraction $A_{d o m, r f}^{s w}$

\begin{tabular}{|c|c|}
\hline Model & Equation \\
\hline CLM4.5 & not represented \\
\hline CLM5.0 & not represented \\
\hline CWatM & not represented \\
\hline $\mathrm{DBH}$ & not represented \\
\hline H08 & $A_{\text {dom }, r f}^{s w}=\left(1-f_{\text {lost }}\right) \times\left(1-f_{\text {cons }, A}\right) \times A_{\text {dom }}^{s w}[24] ;[12]$ \\
\hline JULES-W1 & not represented \\
\hline LPJmL & not represented \\
\hline Mac-PDM.20 & not represented \\
\hline MATSIRO & $\begin{array}{l}90 \% \text { of domestic water use, from surface water bodies, is implicitly assumed to have returned to the original } \\
\text { source (surface water; [39]). }\end{array}$ \\
\hline $\mathrm{mHM}$ & not represented \\
\hline MPI-HM & not represented \\
\hline ORCHIDEE & not represented \\
\hline PCR-GLOBWB & not represented \\
\hline VIC & not represented \\
\hline WaterGAP2 & $A_{d o m, r f}^{s w}=A_{d o m, r f} \times\left(1-f_{g w, u s e}\right)$ \\
\hline & $A_{d o m ~ r f}=\left\{A_{d o m}-\left(A_{d o m} \times C_{d o m, c o n s}\right) \quad\right.$ year $<2000$ \\
\hline & $A_{\text {dom }, r f}= \begin{cases}A_{\text {dom }, r f} & \text { year } \geq 2000\end{cases}$ \\
\hline WAYS & $\begin{array}{l}\text { Starting with } 2000 A_{d o m, r f} \text { is based on wastewater volume data, for details see [18]. } \\
\text { not represented }\end{array}$ \\
\hline
\end{tabular}


Table S65. Equations for livestock groundwater abstraction $A_{l i v}^{g w}$

\begin{tabular}{ll}
\hline Model & Equation \\
\hline CLM4.5 & not represented \\
CLM5.0 & not represented \\
CWatM & not represented \\
DBH & not represented \\
H08 & not represented \\
JULES-W1 & not represented \\
LPJmL & not represented \\
Mac-PDM.20 & not represented \\
MATSIRO & not represented \\
mHM & not represented \\
MPI-HM & not represented \\
ORCHIDEE & not represented \\
PCR-GLOBWB & $A_{l i v}^{g w}=\frac{R_{g w}}{R_{g w}+Q_{i u}} \times A_{l i v, \text { dem }}$ \\
VIC & not represented \\
WaterGAP2 & water used in the livestock sector is solely abstracted from surface water bodies \\
WAYS & not represented \\
\hline
\end{tabular}


Table S66. Equations for livestock groundwater consumption $A_{\text {liv, cons }}^{g w}$

\begin{tabular}{ll}
\hline Model & Equation \\
\hline CLM4.5 & not represented \\
CLM5.0 & not represented \\
CWatM & not represented \\
DBH & not represented \\
H08 & not represented \\
JULES-W1 & not represented \\
LPJmL & not represented \\
Mac-PDM.20 & not represented \\
MATSIRO & not represented \\
mHM & not represented \\
MPI-HM & not represented \\
ORCHIDEE & not represented \\
PCR-GLOBWB & $A_{\text {liv, cons }}^{g w}=\frac{R_{g w}}{R_{g w}+Q_{i u}} \times \frac{A_{l i v, c o n s}}{A_{l i v, \text { dem }}}$ \\
VIC & not represented \\
WaterGAP2 & not represented \\
\hline
\end{tabular}


Table S67. Equations for livestock surface water abstraction $A_{l i v}^{s w}$

\begin{tabular}{ll}
\hline Model & Equation \\
\hline CLM4.5 & not represented \\
CLM5.0 & not represented \\
CWatM & not represented \\
DBH & not represented \\
H08 & not represented \\
JULES-W1 & not represented \\
LPJmL & not represented \\
Mac-PDM.20 & not represented \\
MATSIRO & not represented \\
mHM & not represented \\
MPI-HM & not represented \\
ORCHIDEE & not represented \\
PCR-GLOBWB & $A_{l i v}^{s w}=\frac{Q_{i u}}{R_{g w}+Q_{i u}} \times A_{l i v, d e m}$ \\
VIC & not represented \\
WaterGAP2 & $A_{l i v}^{s w}=A_{l i v, c o n s}^{s w}$ \\
WAYS & not represented \\
\hline
\end{tabular}


Table S68. Equations for livestock surface water consumption $A_{l i v, c o n s}^{s w}$

\begin{tabular}{ll}
\hline Model & Equation \\
\hline CLM4.5 & not represented \\
CLM5.0 & not represented \\
CWatM & not represented \\
DBH & not represented \\
H08 & not represented \\
JULES-W1 & not represented \\
LPJmL & not represented \\
Mac-PDM.20 & not represented \\
MATSIRO & not represented \\
mHM & not represented \\
MPI-HM & not represented \\
ORCHIDEE & not represented \\
PCR-GLOBWB & $A_{\text {liv, cons }}^{\text {sw }}=\frac{Q_{i u}}{R_{g w}+Q_{i u}} \times \frac{A_{l i v, c o n s}}{A_{l i v, d e m}}$ \\
VIC & not represented \\
WaterGAP2 & $A_{l i v, \text { cons }}^{\text {sw }}=\sum_{i=1}^{10} P O P_{l i v, t} \times C_{l i v}^{w, r e q}$ for 10 livestock types [1], [34]. \\
WAYS & not represented \\
\hline
\end{tabular}


Table S69. Equations for manufacturing groundwater abstraction $A_{\text {man }}^{g w}$

\begin{tabular}{|c|c|}
\hline Model & Equation \\
\hline CLM4.5 & not represented \\
\hline CLM5.0 & not represented \\
\hline CWatM & not represented \\
\hline DBH & not represented \\
\hline H08 & $A_{\text {man }}^{g w}=A_{\text {man }}^{g w, r w}+A_{\text {man }}^{g w, n r w}$ \\
\hline & $\begin{array}{l}\text { Where: } \\
A_{m a n}^{g w, r w}=\min \left(f_{g w, u s e} \times A_{m a n, d e m} \times \frac{S_{g w, r w}}{\Delta t}\right)\end{array}$ \\
\hline JULES-W1 & $\begin{array}{l}A_{\text {man }}^{g w, w}=f_{g w, u s e} \times A_{\text {man,dem }}-A_{\text {man }}^{g w, r w} \iota \\
\text { not represented }\end{array}$ \\
\hline LPJmL & not represented \\
\hline Mac-PDM.20 & not represented \\
\hline MATSIRO & $\begin{array}{l}\text { combines manufacturing sector and electricity sector and calls this sector industrial sector. Given the amount } \\
\text { of water requirement for the industrial sector, water is firstly taken from local river flow (potentially regulated } \\
\text { by global reservoir), then from local reservoir (same cell or upstream cell). Then, rest of water requirement } \\
\text { unmet is taken from groundwater resource. Here, here MATSIRO assumes unlimited groundwater resource. } \\
A_{i n d}^{g w}=A_{i n d, d e m}-A_{i n d}^{s w} \text { where } A_{i n d, d e m} \text { is water requirement of the industrial sector. } \\
\text { not represented }\end{array}$ \\
\hline $\mathrm{mHM}$ & not represented \\
\hline MPI-HM & not represented \\
\hline ORCHIDEE & not represented \\
\hline PCR-GLOBWB & $A_{\text {man }}^{g w}=\frac{R_{g w}}{R_{g w}+Q_{i u}} \times A_{m a n, d e m}$ \\
\hline VIC & not represented \\
\hline WaterGAP2 & $A_{\text {man }}^{g w}=A_{\text {man }}^{c} \times \frac{P O P_{u}}{P O P_{u}^{c}} \times f_{g w, u s e}$ \\
\hline WAYS & $\begin{array}{l}A_{\text {man }}^{c}=C_{\text {man }}^{w, i n t, 2005} \times G A V \times C_{\text {man }}^{\text {tech }, c r} \\
\text { not represented }\end{array}$ \\
\hline
\end{tabular}


Table S70. Equations for manufacturing groundwater consumption $A_{\text {man,cons }}^{g w}$

\begin{tabular}{|c|c|}
\hline Model & Equation \\
\hline CLM4.5 & not represented \\
\hline CLM5.0 & not represented \\
\hline CWatM & not represented \\
\hline DBH & not represented \\
\hline JULES-W1 & $\begin{array}{l}A_{\text {man }}^{g w}=A_{\text {man,cons }}^{g w}+A_{\text {man,loss }}^{g w}+A_{\text {man,rf }}^{g w} \\
A_{\text {man,cons }}^{g w}=f_{\text {cons }, A} \times A_{\text {man }}^{g w}[43] \\
A_{\text {man,loss }}^{g w}=f_{\text {lost }} \times\left(1-f_{\text {cons }, A}\right) \times A_{\text {man }}^{g w} \\
\text { not represented }\end{array}$ \\
\hline LPJmL & not represented \\
\hline Mac-PDM.20 & not represented \\
\hline MATSIRO & $\begin{array}{l}10 \% \text { of domestic water use is assumed to be consumptively used. Theoretically speaking: } \\
A_{\text {ind,cons }}^{g w}=X_{\text {ind }} \times A_{i n d}^{g w} \\
\text { where } X_{\text {ind }} \text { is overall water use efficiency of the industrial sector. } \\
\text { not represented }\end{array}$ \\
\hline MPI-HM & not represented \\
\hline ORCHIDEE & not represented \\
\hline PCR-GLOBWB & $A_{\text {man }, \text { cons }}^{g w}=\frac{R_{g w}}{R_{g w}+Q_{i u}} \times \frac{A_{\text {man }, \text { cons }}}{A_{\text {man }, \text { dem }}}$ \\
\hline VIC & not represented \\
\hline WaterGAP2 & $A_{\text {man }, \text { cons }}^{g w}=\left(A_{\text {man }}^{c}-A_{\text {man }, r f}^{c}\right) \times \frac{P O P_{u}}{P O P_{u}^{c}} \times f_{g w, u s e}$ \\
\hline & $\begin{array}{l}A_{\text {man }}^{c}=C_{\text {man }}^{w, \text { ints }, 2005} \times G A V \times C_{\text {man }}^{\text {tech }, \text { cr }} \\
A_{\text {man }, r f}^{c}= \begin{cases}A_{\text {man }}^{c} \times C_{\text {man }, \text { cons }} & \text { year }<2000 \\
A_{\text {man }}^{\text {coo, }}+A_{\text {man }}^{\text {ww,c }} & \text { year } \geq 2000\end{cases} \end{array}$ \\
\hline WAYS & $\begin{array}{l}\text { For details on manufacturing cooling and waste water see [18]. } \\
\text { not represented }\end{array}$ \\
\hline
\end{tabular}


Table S71. Equations for return flow from manufacturing groundwater abstraction $A_{\text {man,rf }}^{g w}$

\begin{tabular}{|c|c|}
\hline Model & Equation \\
\hline CLM4.5 & not represented \\
\hline CLM5.0 & not represented \\
\hline CWatM & not represented \\
\hline DBH & not represented \\
\hline H08 & $A_{\text {man }, r f}^{g w}=\left(1-f_{\text {lost }}\right) \times\left(1-f_{\text {cons }, A}\right) \times A_{\text {man }}^{g w}$ \\
\hline JULES-W1 & not represented \\
\hline LPJmL & not represented \\
\hline Mac-PDM.20 & not represented \\
\hline $\begin{array}{l}\text { MATSIRO } \\
\text { mHM }\end{array}$ & $\begin{array}{l}90 \% \text { of domestic water use is implicitly assumed to have returned to the original source (groundwater; [39]). } \\
\text { not represented }\end{array}$ \\
\hline MPI-HM & not represented \\
\hline ORCHIDEE & not represented \\
\hline PCR-GLOBWB & $A_{m a n, r f}^{g w}=\frac{R_{g w}}{R+O} \times \frac{A_{m a n, d e m}-A_{m a n, c o n s}}{A}$ \\
\hline VIC & not represented \\
\hline WaterGAP2 & $A_{\text {man }, r f}^{g w}= \begin{cases}A_{\text {man }}^{g w} \times C_{\text {man }, \text { cons }}, & \text { year }<2000 \\
\left(A_{\text {man }}^{\text {coo,c }}+A_{\text {man }}^{\text {ww }, c}\right) \times \frac{P O P_{u}}{P O P^{c}} \times f_{g w, \text { use }}, & \text { year } \geq 2000\end{cases}$ \\
\hline WAYS & $\begin{array}{l}\text { For details on manufacturing cooling and waste water see [18]. } \\
\text { not represented }\end{array}$ \\
\hline
\end{tabular}


Table S72. Equations for manufacturing surface water abstraction $A_{\text {man }}^{s w}$

\begin{tabular}{|c|c|}
\hline Model & Equation \\
\hline CLM4.5 & not represented \\
\hline CLM5.0 & not represented \\
\hline CWatM & not represented \\
\hline $\mathrm{DBH}$ & not used for ISIMIP2b \\
\hline H08 & $A_{\text {man }}^{s w}=A_{\text {man }}^{a q}+A_{\text {man }}^{r e}+A_{\text {man }}^{r i}+A_{\text {man }}^{u s w}$ \\
\hline JULES-W1 & not represented \\
\hline LPJmL & prescribed data offered by ISIMIP2b \\
\hline Mac-PDM.20 & not represented \\
\hline MATSIRO & combines manufacturing sector and electricity sector and calls this sector industrial sector. \\
\hline & $\begin{array}{l}\text { Given the amount of water requirement for this sector, water is firstly taken from local river flow (potentially } \\
\text { regulated by global reservoir), then from local reservoir (same cell or upstream cell). } \\
A_{\text {ind }}^{\text {sw }}=A_{\text {ind }}^{\text {pond }}+A_{\text {ind }}^{r e, g l o b a l}+A_{\text {ind }}^{r i}\end{array}$ \\
\hline $\mathrm{mHM}$ & not represented \\
\hline MPI-HM & not represented \\
\hline ORCHIDEE & not represented \\
\hline PCR-GLOBWB & $A_{m a n}^{s w}=\frac{Q_{i u}}{R_{g w}+Q_{i u}} \times A_{m a n, d e m}$ \\
\hline VIC & not represented \\
\hline WaterGAP2 & $A_{\text {man }}^{s w}=A_{\text {man }}^{c} \times \frac{P O P_{u}}{P O P_{u}^{c}} \times\left(1-f_{g w, u s e}\right)$ \\
\hline WAYS & 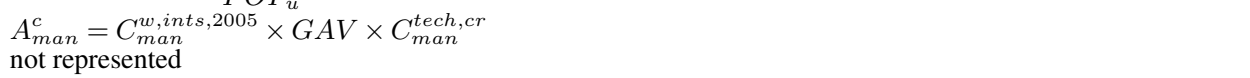 \\
\hline
\end{tabular}


Table S73. Equations for manufacturing surface water consumption $A_{\text {man,cons }}^{s w}$

\begin{tabular}{|c|c|}
\hline Model & Equation \\
\hline CLM4.5 & not represented \\
\hline CLM5.0 & not represented \\
\hline CWatM & not represented \\
\hline $\mathrm{DBH}$ & not represented \\
\hline H08 & 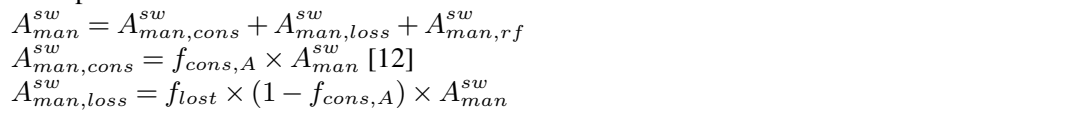 \\
\hline JULES-W1 & See [12] \\
\hline LPJmL & prescribed data offered by ISIMIP2b \\
\hline Mac-PDM.20 & not represented \\
\hline MATSIRO & $\begin{array}{l}\text { only } 10 \% \text { of domestic water use is assumed to be consumptively used. Theoretically speaking: } \\
A_{i n d, c o n s}^{\text {sw }}=X_{\text {ind }} \times A_{i n d}^{\text {sw }}\end{array}$ \\
\hline mHM & not represented \\
\hline MPI-HM & not represented \\
\hline ORCHIDEE & not represented \\
\hline PCR-GLOBWB & $A_{\text {man }, \text { cons }}^{\text {sw }}=\frac{Q_{i u}}{R} \times \frac{A_{\text {man }, \text { cons }}}{A}$ \\
\hline VIC & $\begin{array}{l}R_{g w}+Q_{i u} \quad A_{\text {man }, d e m} \\
\text { not represented }\end{array}$ \\
\hline \multirow[t]{4}{*}{ WaterGAP2 } & $A_{m a n, c o n s}^{s w}=\left(A_{m a n}^{c}-A_{m a n, r f}^{c}\right) \times \frac{P O P_{u}}{P O P_{u}^{c}} \times\left(1-f_{g w, u s e}\right)$ \\
\hline & $A_{\text {man }}^{c}=C_{m a n}^{w, i n t s, 2005} \times G A V \times C_{m a n}^{\text {tech,cr }}$ \\
\hline & $A^{c}=\int A_{\text {man }}^{c} \times C_{\text {man,cons }}$ year $<2000$ \\
\hline & $A_{\text {man }, r f}= \begin{cases}A_{\text {man }}^{c o o, c}+A_{\text {man }}^{\text {ww,c }} & \text { year } \geq 2000\end{cases}$ \\
\hline WAYS & $\begin{array}{l}\text { For details on manufacturing cooling and waste water see [18]. } \\
\text { not represented }\end{array}$ \\
\hline
\end{tabular}


Table S74. Equations for return flow from manufacturing surface water abstraction $A_{\text {man,rf }}^{s w}$

\begin{tabular}{|c|c|}
\hline Model & Equation \\
\hline CLM4.5 & not represented \\
\hline CLM5.0 & not represented \\
\hline CWatM & not represented \\
\hline $\mathrm{DBH}$ & not represented \\
\hline $\mathrm{H} 08$ & $A_{\text {man }, r f}^{s w}=\left(1-f_{\text {lost }}\right) \times\left(1-f_{\text {cons }, A}\right) \times A_{\text {man }}^{\text {sw }}$. See [24] \\
\hline JULES-W1 & not represented \\
\hline LPJmL & not represented \\
\hline Mac-PDM.20 & not represented \\
\hline MATSIRO & $90 \%$ of domestic water use is implicitly assumed to have returned to the original source (surface water; [39]). \\
\hline mHM & not represented \\
\hline MPI-HM & not represented \\
\hline ORCHIDEE & not represented \\
\hline PCR-GLOBWB & not represented \\
\hline VIC & not represented \\
\hline & $A^{s w} \quad \int A_{m a n}^{s w} \times C_{m a n, c o n s}$ \\
\hline wateruAPL & $A_{\text {man }, r f}=\left\{\begin{array}{l}\left(A_{\text {man }}^{\text {coo,c }}+A_{\text {man }}^{w w, c}\right) \times \frac{P O P_{u}}{P O P_{u}^{c}} \times\left(1-f_{g w, u s e}\right) \quad \text { year } \geq 2000\end{array}\right.$ \\
\hline WAYS & $\begin{array}{l}\text { For details on manufacturing cooling and waste water see [18]. } \\
\text { not represented }\end{array}$ \\
\hline
\end{tabular}


Table S75. Equations for electricity surface water abstraction

\begin{tabular}{ll}
\hline Model & Equation \\
\hline CLM4.5 & not represented \\
CLM5.0 & not represented \\
CWatM & not represented \\
DBH & not represented \\
H08 & not represented \\
JULES-W1 & not represented \\
LPJmL & not represented \\
Mac-PDM.20 & not represented \\
MATSIRO & nepresented in table S72. \\
mHM & not represented \\
MPI-HM & not represented \\
ORCHIDEE & not represented \\
PCR-GLOBWB & not represented \\
VIC & not represented \\
WaterGAP2 & $A_{\text {ele }}^{\text {sw }} \sum_{i=1}^{n} J_{\text {ele }, \text { prod }, i} \times J_{\text {ele }, \text { A }, \text { ints }, i}\left(J_{\text {ele }, \text { coo }, i}, J_{\text {ele }, p t, i}\right) \times C_{\text {ele }}^{\text {techangerate }}$ \\
WAYS & not represented \\
\hline
\end{tabular}


Table S76. Equations for electricity surface water consumption

\begin{tabular}{|c|c|}
\hline Model & Equation \\
\hline CLM4.5 & not represented \\
\hline CLM5.0 & not represented \\
\hline CWatM & not represented \\
\hline DBH & not represented \\
\hline H08 & not represented \\
\hline JULES-W1 & not represented \\
\hline LPJmL & not represented \\
\hline Mac-PDM.20 & not represented \\
\hline MATSIRO & not represented \\
\hline mHM & not represented \\
\hline MPI-HM & not represented \\
\hline ORCHIDEE & not represented \\
\hline PCR-GLOBWB & not represented \\
\hline VIC & not represented \\
\hline WaterGAP2 & $A_{\text {ele }}^{s w}=\sum_{i=1}^{n} J_{\text {ele }, \text { prod }, i} \times J_{\text {ele }, \text { cons }, \text { ints }, i}\left(J_{\text {ele }, \text { coo }, i}, J_{\text {ele }, p t, i}\right) \times C_{\text {ele }}^{\text {techchangerate }}$ \\
\hline WAYS & not represented \\
\hline
\end{tabular}


Table S77. Groundwater abstraction $A_{g w}$

\begin{tabular}{ll}
\hline Model & Equation \\
\hline CLM4.5 & not represented \\
CLM5.0 & not represented \\
CWatM & not represented \\
DBH & not represented \\
H08 & not represented \\
JULES-W1 & not represented \\
LPJmL & not represented \\
Mac-PDM.20 & not represented \\
MATSIRO & $A_{g w}=A_{i r r}^{g w}-A_{d o m}^{g w}-A_{i n d}^{g w}$ \\
mHM & not represented \\
MPI-HM & $A_{g w}=A_{i r r}^{g w}$ \\
ORCHIDEE & not represented \\
PCR-GLOBWB & not represented \\
VIC & not represented \\
WaterGAP2 & $A_{g w}=A_{i r r}^{g w}-A_{d o m}^{g w}-A_{i n d}^{g w}$ \\
WAYS & not represented \\
\hline
\end{tabular}


Table S78. Total lake abstraction $A_{l a}$

\begin{tabular}{|c|c|}
\hline Model & Equation \\
\hline CLM4.5 & not represented \\
\hline CLM5.0 & not represented \\
\hline CWatM & not represented \\
\hline DBH & not represented \\
\hline $\mathrm{H} 08$ & not represented \\
\hline JULES-W1 & not represented \\
\hline LPJmL & $A_{l a}=\left(A_{\text {irr }, \text { gross }, G}+A_{\text {hil }, G}\right)+\left(A_{\text {irr }, \text { gross }, \text { Gdownstream }}+A_{\text {hil }, \text { Gdownstream }}\right)$ \\
\hline Mac-PDM.20 & not represented \\
\hline MATSIRO & not represented \\
\hline $\mathrm{mHM}$ & not represented \\
\hline MPI-HM & $A_{l a}=A_{i r r}^{s w}$ \\
\hline ORCHIDEE & not represented \\
\hline PCR-GLOBWB & $A_{l a}=A_{d o m, d e m}+A_{\text {ind }, d e m}+A_{i r r, d e m}+A_{l i v, d e m}$ \\
\hline VIC & not represented \\
\hline WaterGAP2 & $A_{l a}=A_{d o m}^{s w}+A_{e l e}^{s w}+A_{i r r}^{s w}+A_{l i v}^{s w}+A_{m a n}^{s w}$ \\
\hline WAYS & $\begin{array}{l}\text { The net surface water abstraction is satisfied in WaterGAP2 in following order 1. River, 2. global lakes and } \\
\text { reservoirs and } 3 \text {. local lakes } \\
\text { not represented }\end{array}$ \\
\hline
\end{tabular}


Table S79. Total reservoir abstraction $A_{r e}$

\begin{tabular}{|c|c|}
\hline Model & Equation \\
\hline CLM4.5 & not represented \\
\hline CLM5.0 & not represented \\
\hline CWatM & not represented \\
\hline $\mathrm{DBH}$ & not used for ISIMIP2b \\
\hline H08 & $A_{r e}=A_{\text {dom }, \text { month }}+A_{\text {ind,month }}+A_{\text {irr }, \text { month }}$ \\
\hline JULES-W1 & not represented \\
\hline LPJmL & $A_{r e}=\left(A_{\text {irr }, \text { gross }, G}+A_{\text {hil }, G}\right)+\left(A_{\text {irr }, \text { gross }, \text { Gdownstream }}+A_{\text {hil }, \text { Gdownstream }}\right)$ \\
\hline Mac-PDM.20 & not represented \\
\hline MATSIRO & $A_{r e}=A_{d o m}^{r e}+A_{i n d}^{r e}+A_{i r r}^{r e}$ \\
\hline $\mathrm{mHM}$ & not represented \\
\hline MPI-HM & not represented \\
\hline ORCHIDEE & not represented \\
\hline PCR-GLOBWB & $A_{l a}=A_{d o m, d e m}+A_{i n d, d e m}+A_{i r r, d e m}+A_{l i v, d e m}$ \\
\hline VIC & not represented \\
\hline \multirow[t]{2}{*}{ WaterGAP2 } & $A_{r e}=A_{d o m}^{s w}+A_{e l e}^{s w}+A_{i r r}^{s w}+A_{l i v}^{s w}+A_{m a n}^{s w}$ \\
\hline & $\begin{array}{l}\text { The net surface water abstraction is satisfied in WaterGAP2 in following order 1. River, 2. global lakes and } \\
\text { reservoirs and 3. local lakes }\end{array}$ \\
\hline WAYS & S \\
\hline
\end{tabular}


Table S80. Total river abstraction $A_{r i}$

\begin{tabular}{ll}
\hline Model & Equation \\
\hline CLM4.5 & not represented \\
CLM5.0 & $A_{r i}=A_{\text {irr }}$ \\
CWatM & not represented \\
DBH & not represented \\
H08 & not represented \\
JULES-W1 & not represented \\
LPJmL & not represented \\
Mac-PDM.20 & not represented \\
MATSIRO & not represented \\
mHM & not represented \\
MPI-HM & combined with lake abstraction \\
ORCHIDEE & not represented \\
PCR-GLOBWB & not represented \\
VIC & not represented \\
WaterGAP2 & $A_{r i}=A_{\text {dom }}^{s w}+A_{\text {ele }}^{s w}+A_{i r r}^{s w}+A_{\text {liv }}^{s w}+A_{\text {man }}^{s w}$ \\
& The net surface water abstraction is satisfied in WaterGAP2 in following order 1. River, 2. global lakes and \\
WAYS & reservoirs and 3. local lakes \\
\hline
\end{tabular}


Table S81. Seawater abstraction $A_{\text {ocean }}$

\begin{tabular}{ll}
\hline Model & Equation \\
\hline CLM4.5 & not represented \\
CLM5.0 & not represented \\
CWatM & not represented \\
DBH & not represented \\
H08 & To have the potential to use desalination three conditions must be met: \\
& 1) GDP > 14000 USD person / year in terms of purchasing power parity (PPP) \\
& 2) humidity index below $8 \%$ \\
& 3) within 3 grid cells of the seashore \\
& It is assumed that seawater desalination is not used for irrigation and all demand for municipal and industrial \\
JULES-W1 & water is abstracted by desalination if available. Therefore: \\
LPJmL & Aocean $=A_{\text {ind }}+A_{\text {muni }}$ \\
Mac-PDM.20 & not represented \\
MATSIRO & not represented \\
mHM & not represented \\
MPI-HM & not represented \\
ORCHIDEE & not represented \\
PCR-GLOBWB & not represented \\
VIC & not represented \\
WaterGAP2 & not represented \\
WAYS & not represented \\
\hline
\end{tabular}


Table S82. Seawater consumption $A_{\text {ocean,cons }}$

\begin{tabular}{ll}
\hline Model & Equation \\
\hline CLM4.5 & not represented \\
CLM5.0 & not represented \\
CWatM & not represented \\
DBH & not represented \\
H08 & Desalination is not used for irrigation. \\
& $A_{\text {ocean, cons }}=f_{\text {cons }, A} \times A_{\text {ocean }}$ \\
& Where $f_{\text {cons }, A}$ is the ratio of consumption to withdrawal and is equal to 0.1 and 0.15 for industrial and municipal \\
JULES-W1 & water use, respectively. \\
LPJmL & not represented \\
Mac-PDM.20 & not represented \\
MATSIRO & not represented \\
mHM & not represented \\
MPI-HM & not represented \\
ORCHIDEE & not represented \\
PCR-GLOBWB & not represented \\
VIC & not represented \\
WaterGAP2 & not represented \\
WAYS & not represented \\
\hline
\end{tabular}


Table S83. Return flow from seawater abstraction $A_{\text {ocean }, r f}$

\begin{tabular}{ll}
\hline Model & Equation \\
\hline CLM4.5 & not represented \\
CLM5.0 & not represented \\
CWatM & not represented \\
DBH & not represented \\
H08 & Desalination is never used for irrigation. \\
& $A_{\text {ocean, } r f}=\left(1-f_{\text {lost }}\right) \times\left(1-f_{\text {cons }, A}\right) \times A_{\text {ocean }}$ \\
& Where, $f_{\text {cons }, A}$ is the ratio of consumption to withdrawal $(-)$ and $f_{\text {lost }}$ is the proportion lost during delivery (-). \\
& The fist factor is set to 0.1 for industrial use and 0.15 for municipal use the 0. \\
JULES-W1 & not represented \\
LPJmL & not represented \\
Mac-PDM.20 & not represented \\
MATSIRO & not represented \\
mHM & not represented \\
MPI-HM & not represented \\
ORCHIDEE & not represented \\
PCR-GLOBWB & not represented \\
VIC & not represented \\
WaterGAP2 & not represented \\
WAYS & not represented \\
\hline
\end{tabular}




\section{References}

[1] Alcamo, J., Döll, P., Henrichs, T., Kaspar, F., Lehner, B., Rösch, T., and Siebert, S.: Development and testing of the WaterGAP 2 global model of water use and availability, Hydrological Sciences Journal, 48, 317-338, https://doi.org/10.1623/hysj.48.3.317.45290, 2003.

[2] Andreadis, K. M., Storck, P., and Lettenmaier, D. P.: Modeling snow accumulation and ablation processes in forested environments, Water Resources Research, 45, W05 429, https://doi.org/10.1029/2008WR007042, URL https://agupubs. onlinelibrary.wiley.com/doi/abs/10.1029/2008WR007042, 2009.

[3] Bergström, S.: The HBV model, in: Computer models of watershed hydrology, edited by Singh, V., pp. 443-476, Water Resources Publications, Lone Tree, USA, 1995.

[4] Best, J. R., Miller, P. H., and Jones, L. L.: Executive functions after age 5: Changes and correlates., Developmental review : DR, 29, 180-200, https://doi.org/10.1016/j.dr.2009.05.002, 2009.

[5] Best, M. J., Pryor, M., Clark, D. B., Rooney, G. G., Essery, R. L. H., Ménard, C. B., Edwards, J. M., Hendry, M. A., Porson, A., Gedney, N., Mercado, L. M., Sitch, S., Blyth, E., Boucher, O., Cox, P. M., Grimmond, C. S. B., and Harding, R. J.: The Joint UK Land Environment Simulator (JULES), model description - Part 1: Energy and water fluxes, Geoscientific Model Development, https://doi.org/10.5194/gmd-4-677-2011, 2011.

[6] Blondin, C.: Parameterization of Land-Surface Processes in Numerical Weather Prediction, in: Land Surface Evaporation, edited by Schmugge, T. and Andre, J., pp. 31-54, Springer-Verlag, New York, https://doi.org/10.1007/978-1-4612-30328_3, 1991 .

[7] Brouwer, C., Prins, K., and Heibloem, M.: Irrigation water management: irrigation scheduling, Training manual No. 4, FAO Land and Water Development Division, Rome, 1989.

[8] Cherkauer, K. A. and Lettenmaier, D. P.: Hydrologic effects of frozen soils in the upper Mississippi River basin, Journal of Geophysical Research: Atmospheres, 104, 19599-19610, https://doi.org/10.1029/1999JD900337, 1999.

[9] Chow, V. T., Maidment, D. R., and Mays, L. W.: Applied hydrology, McGraw-Hill, New York, 1998.

[10] Clapp, R. B. and Hornberger, G. M.: Empirical equations for some soil hydraulic properties, Water Resources Research, 14, 601-604, https://doi.org/10.1029/WR014i004p00601, URL https://agupubs.onlinelibrary.wiley.com/doi/abs/10.1029/ WR014i004p00601, 1978.

[11] Döll, P. and Fiedler, K.: Global-scale modeling of groundwater recharge, Hydrology and Earth System Sciences, 12, 863-885, https://doi.org/10.5194/hess-12-863-2008, URL http://www.hydrol-earth-syst-sci.net/12/863/2008/, 2008.

[12] Döll, P. and Siebert, S.: Global modeling of irrigation water requirements, Water Resources Research, 38, 8-1, https://doi.org/10.1029/2001wr000355, 2002.

[13] Döll, P., Kaspar, F., and Lehner, B.: A global hydrological model for deriving water availability indicators: Model tuning and validation, Journal of Hydrology, 270, 105-134, https://doi.org/10.1016/S0022-1694(02)00283-4, 2003.

[14] Döll, P., Fiedler, K., and Zhang, J.: Global-scale analysis of river flow alterations due to water withdrawals and reservoirs, Hydrology and Earth System Sciences, 13, 2413-2432, https://doi.org/10.5194/hess-13-2413-2009, 2009.

[15] D'Orgeval, T., Polcher, J., and de Rosnay, P.: Sensitivity of the West African hydrological cycle in ORCHIDEE to infiltration processes, Hydrology and Earth System Sciences, 12, 1387-1401, https://doi.org/10.5194/hess-12-1387-2008, 2008. 
[16] Ducoudré, N. I., Laval, K., and Perrier, A.: SECHIBA, a new set of parameterizations of the hydrologic exchanges at the land-atmosphere interface within the LMD atmospheric general circulation model, Journal of Climate, 6, 248-273, https://doi.org/10.1175/1520-0442(1993)006<0248:SANSOP>2.0.CO;2, 1993.

[17] Essery, R., Pomeroy, J., Parviainen, J., and Storck, P.: Sublimation of snow from coniferous forests in a climate model, Journal of Climate, 16, 1855-1864, https://doi.org/10.1175/1520-0442(2003)016<1855:SOSFCF>2.0.CO;2, 2003.

[18] Flörke, M., Kynast, E., Bärlund, I., Eisner, S., Wimmer, F., and Alcamo, J.: Domestic and industrial water uses of the past 60 years as a mirror of socio-economic development: A global simulation study, Global Environmental Change, 23, 144-156, https://doi.org/10.1016/j.gloenvcha.2012.10.018, 2013.

[19] Franchini, M. and Pacciani, M.: Comparative analysis of several conceptual rainfall-runoff models, Journal of Hydrology, 122, 161-219, https://doi.org/10.1016/0022-1694(91)90178-k, 1991.

[20] Gao, H., Tang, Q., Shi, X., Zhu, C., Bohn, T., and Su, F.: Water budget record from variable infiltration capacity (VIC) model, in: Algorithm Theoretical Basis Document for Terrestrial Water Cycle Data Records, pp. 120-173, UNSPECIFIED, 2010.

[21] Gerten, D., Schaphoff, S., Haberlandt, U., Lucht, W., and Sitch, S.: Terrestrial vegetation and water balance-hydrological evaluation of a dynamic global vegetation model, Journal of Hydrology, 286, 249270, https://doi.org/https://doi.org/10.1016/j.jhydrol.2003.09.029, URL http://www.sciencedirect.com/science/article/pii/ S0022169403003901, 2004.

[22] Gregory, D., S. R. C. P.: Canopy, surface and soil hydrology, Tech. rep., Climate Research Meteorological Office, London, 1994.

[23] Hanasaki, N., Kanae, S., and Oki, T.: A reservoir operation scheme for global river routing models, Journal of Hydrology, 327, 22-41, https://doi.org/10.1016/j.jhydrol.2005.11.011, 2006.

[24] Hanasaki, N., Yoshikawa, S., Pokhrel, Y., and Kanae, S.: A global hydrological simulation to specify the sources of water used by humans, Hydrology and Earth System Sciences, 22, 789-817, https://doi.org/10.5194/hess-22-789-2018, 2018.

[25] Hargreaves, G. and Samani, Z.: Estimating potential evapotranspiration, Journal of Irrigation and Drainage Engineering, 108, 223-230, 1982.

[26] Kaspar, F.: Entwicklung und Unsicherheitsanalyse eines globalen hydrologischen Modells, Ph.D. thesis, University of Kassel, Kassel, 2003.

[27] Koirala, K., Mishra, A., and Mohanty, S.: Determinants of rice productivity and technical efficiency in the Philippines, in: Southern Agricultural Economics Association (SAEA) Annual Meeting, pp. 1-19, Dallas, https://doi.org/10.13140/2.1.3275.1360, 2014.

[28] Liang, X., Lettenmaier, D., Wood, E., and Burges, S.: A simple hydrologically based model of land-surface water and enery fluxes for general-circulation models, Journal of Geophysical Research Atmospheres, 99, 14415-14 428, https://doi.org/10.1029/94JD00483, 1994.

[29] Liang, X., Wood, E. F., and Lettenmaier, D. P.: Surface soil moisture parameterization of the VIC-2L model: Evaluation and modification, Global and Planetary Change, https://doi.org/10.1016/0921-8181(95)00046-1, 1996.

[30] Maniak, U.: Hydrologie und Wasserwirtschaft, Springer-Verlag, Berlin Heidelberg, https://doi.org/10.1007/3-540-278397, 1997.

[31] Milly, P. C. D.: Potential evaporation and soil moisture in general circulation models, Journal of Climate, 5, 209-226, https://doi.org/10.1175/1520-0442(1992)005<0209:PEASMI>2.0.CO;2, 1992. 
[32] Molnau, M. and Bissell, V.: A continuous frozen ground index for flood forecasting, 1983.

[33] Monteith, J.: MonteithEvaporationandenvironment1965, Symposia of the Society for Experimental Biology, pp. 205-234, 1965.

[34] Müller Schmied, H., Eisner, S., Franz, D., Wattenbach, M., Portmann, F. T., Flörke, M., and Döll, P.: Sensitivity of simulated global-scale freshwater fluxes and storages to input data, hydrological model structure, human water use and calibration, Hydrology and Earth System Sciences, 18, 3511-3538, https://doi.org/10.5194/hess-18-3511-2014, 2014.

[35] Nachtergaele, F., von Velthuizen, H., Vereist, L., Batjes, N. H., Dijkshoorn, J. A., van Engelen, V. W. P., Fischer, G., Jones, A., Montanarella, L., Petri, M., Prieler, S., Teixeira, E., Wilberg, D., and Shi, X.: Harmonized world soil database (version 1.0), FAO, Rome, Italy and IIASA, Laxenburg, Austria, 2008.

[36] Oki, T., Nishimura, T., and Dirmeyer, P.: Assessment of annual runoff from land surface models using total runoff integrating pathways (TRIP), Journal of the Meteorological Society of Japan, 77, 235-255, https://doi.org/10.2151/jmsj1965.77.1B_235, 1999.

[37] Oleson, K., Lawrence, D., Bonan, G., Drewniak, B., Huang, M., Koven, C., Levis, S., Li, F., Riley, W., Subin, Z., Swenson, S., Thornton, P., Bozbiyik, A., Fisher, R., Heald, C., Kluzek, E., Lamarque, J.-F., Lawrence, P., Leung, L., and Yang, Z.-L.: Technical description of version 4.5 of the Community Land Model (CLM), Tech. rep., National Center for Atmospheric Research, Boulder, Colorado, https://doi.org/10.5065/D6RR1W7M, 2013.

[38] Pokhrel, Y., Hanasaki, N., Koirala, S., Cho, J., Yeh, P. J., Kim, H., Kanae, S., and Oki, T.: Incorporating anthropogenic water regulation modules into a land surface model, Journal of Hydrometeorology, 13, 255-269, https://doi.org/10.1175/JHM-D-11-013.1, 2012.

[39] Pokhrel, Y. N., Koirala, S., Yeh, P. J., Hanasaki, N., Longuevergne, L., Kanae, S., and Oki, T.: Incorporation of groundwater pumping in a global Land Surface Model with the representation of human impacts, Water Resources Research, 51, 78-96, https://doi.org/10.1002/2014WR015602, 2015.

[40] Robock, A., Vinnikov, K. Y., Schlosser, C. A., Speranskaya, N. A., and Xue, Y.: Use of midlatitude soil moisture and meteorological observations to validate soil moisture simulations with biosphere and bucket models, Journal of Climate, 8, 15-35, https://doi.org/10.1175/1520-0442(1995)008<0015:UOMSMA>2.0.CO;2, 1995.

[41] Rogers, D. H., Lamm, F. R., Alam, M., Troolen, T. P., Clark, G. A., Barnes, P. L., and Mankin, K.: Efficiencies and water losses of irrigation systems, Irrigation Management Series, MF-2243, 1997.

[42] Schulze, K. and Döll, P.: Neue Ansätze zur Modellierung von Schneeakkumulation und -schmelze im globalen Wassermodell WaterGAP, Tagungsband zum 7. Workshop zur großskaligen Modellierung in der Hydrologie, edited by: Ludwig, R., Reichert, D., and Mauser, W., Kassel University Press, Kassel, 2004.

[43] Shiklomanov, I. A.: Appraisal and Assessment of world water resources, Water International, 25, 11-32, https://doi.org/10.1080/02508060008686794, 2000.

[44] Smirnova, T., Brown, J., Benjamin, S., and Kim, D.: Parameterization of cold-season processes in the MAPS land-surface scheme, Journal of Geophysical Research, 105, 4077-4086, https://doi.org/10.1029/1999JD901047, 2000.

[45] Speers, D. D. and Versteeg, J. D.: Runoff forecasting for reservoir operations - the past and the future, 1979.

[46] Steduto, P., Hsiao, T. C., Fereres, E., and Raes, D.: Crop yield response to water, Irrigation and Drainage Paper No. 66, FAO, Rome, URL http://www.fao.org/docrep/016/i2800e/i2800e.pdf, 2012.

890 [47] Storck, P.: Trees, snow and flooding: An investigation of forest canopy effects on snow accumulation and melt at the plot and watershed scales in the Pacific Northwest, Water Resources Series Technical Report, 161, 176, 2000. 
[48] Storck, P., Lettenmaier, D. P., and Bolton, S. M.: Measurement of snow interception and canopy effects on snow accumulation and melt in a mountainous maritime climate, Oregon, United States, Water Resources Research, 38, 5-16, https://doi.org/10.1029/2002WR001281, 2002.

[49] Takata, K., Emori, S., and Watanabe, T.: Development of the minimal advanced treatments of surface interaction and runoff, Global and Planetary Change, 38, 209-222, https://doi.org/10.1016/S0921-8181(03)00030-4, 2003.

[50] Thober, S., Cuntz, M., Kelbling, M., Kumar, R., Mai, J., and Samaniego, L.: The multiscale routing model mRM v1.0: simple river routing at resolutions from 1 to $50 \mathrm{~km}$, Geoscientific Model Development Discussions, 12, 2501-2521, https://doi.org/10.5194/gmd-12-2501-2019, 2019.

900 [51] Van Diepen, C., Rappoldt, C., and Wolf, J.: CWFS crop growth simulation model WOFOST documentation: version 4.1, Centre for World Food Studies, Amsterdam, 1988.

[52] Verzano, K., Bärlund, I., Flörke, M., Lehner, B., Kynast, E., Voß, F., and Alcamo, J.: Modeling variable river flow velocity on continental scale: Current situation and climate change impacts in Europe, Journal of Hydrology, 424-425, 238-251, https://doi.org/10.1016/j.jhydrol.2012.01.005, 2012.

[53] Wang, T., Ottlé, C., Boone, A., Ciais, P., Brun, E., Morin, S., Krinner, G., Piao, S., and Peng, S.: Evaluation of an improved intermediate complexity snow scheme in the ORCHIDEE land surface model, Journal of Geophysical Research Atmospheres, 118, 6064-6079, https://doi.org/10.1002/jgrd.50395, 2013.

[54] Wang-Erlandsson, L., van der Ent, R. J., Gordon, L. J., and Savenije, H. H. G.: Contrasting roles of interception and transpiration in the hydrological cycle - Part 1: Temporal characteristics over land, Earth System Dynamics, 5, 441-469, https://doi.org/10.5194/esd-5-441-2014, 2014.

[55] Yeh, P.-F. and Eltahir, E.: Representation of water table dynamics in a land surface scheme. Part II: Subgrid variability, Journal of Climate, 18, 1881-1901, 2005. 
Table S84. Glossary with variables used in the study

\begin{tabular}{|c|c|c|}
\hline Symbol & $\begin{array}{l}\text { Dimension } \\
\text { ( } \mathrm{L}=\text { length; } \\
\mathrm{T}=\text { time })\end{array}$ & Variable: with synonyms \\
\hline$A$ & $\mathrm{~L}^{3} \mathrm{~L}^{-2} \mathrm{~T}^{-1}$ & $\begin{array}{l}\text { water abstraction: water } \\
\text { withdrawal }\end{array}$ \\
\hline$A_{\text {pot }}$ & $\mathrm{L}^{3} \mathrm{~L}^{-2} \mathrm{~T}^{-1}$ & $\begin{array}{l}\text { potential water abstraction: } \\
\text { potential water withdrawal }\end{array}$ \\
\hline$A_{\text {act }}$ & $\mathrm{L}^{3} \mathrm{~L}^{-2} \mathrm{~T}^{-1}$ & actual water abstraction: \\
\hline$A_{\text {cons }}$ & $\mathrm{L}^{3} \mathrm{~L}^{-2} \mathrm{~T}^{-1}$ & $\begin{array}{l}\text { actual water withdrawal } \\
\text { water consumption: water } \\
\text { use }\end{array}$ \\
\hline$A_{\mathrm{dom}}$ & $\mathrm{L}^{3} \mathrm{~L}^{-2} \mathrm{~T}^{-1}$ & $\begin{array}{l}\text { domestic water abstraction: } \\
\text { domestic water withdrawal }\end{array}$ \\
\hline$A_{\text {ele }}$ & $\mathrm{L}^{3} \mathrm{~L}^{-2} \mathrm{~T}^{-1}$ & $\begin{array}{l}\text { electricity water } \\
\text { abstraction: electricity } \\
\text { water withdrawal }\end{array}$ \\
\hline$A_{\text {irr }}$ & $\mathrm{L}^{3} \mathrm{~L}^{-2} \mathrm{~T}^{-1}$ & $\begin{array}{l}\text { irrigation water abstraction: } \\
\text { irrigation water withdrawal }\end{array}$ \\
\hline$A_{\text {ind }}$ & $\mathrm{L}^{3} \mathrm{~L}^{-2} \mathrm{~T}^{-1}$ & $\begin{array}{l}\text { industrial water abstraction: } \\
\text { industrial water withdrawal }\end{array}$ \\
\hline$A_{\text {liv }}$ & $\mathrm{L}^{3} \mathrm{~L}^{-2} \mathrm{~T}^{-1}$ & livestock water abstraction: \\
\hline$A_{\text {man }}$ & $\mathrm{L}^{3} \mathrm{~L}^{-2} \mathrm{~T}^{-1}$ & $\begin{array}{l}\text { livestock water withdrawal } \\
\text { manufacturing water } \\
\text { abstraction: manufacturing } \\
\text { water withdrawal }\end{array}$ \\
\hline$A_{\mathrm{rf}}$ & $\mathrm{L}^{3} \mathrm{~L}^{-2} \mathrm{~T}^{-1}$ & $\begin{array}{l}\text { return flow from water } \\
\text { abstraction: return flow } \\
\text { from water withdrawal }\end{array}$ \\
\hline$A^{\text {oc }}$ & $\mathrm{L}^{3} \mathrm{~L}^{-2} \mathrm{~T}^{-1}$ & $\begin{array}{l}\text { seawater abstraction: } \\
\text { seawater withdrawal }\end{array}$ \\
\hline$E_{\text {so }}$ & $\mathrm{L}^{3} \mathrm{~L}^{-2} \mathrm{~T}^{-1}$ & $\begin{array}{l}\text { evaporation from soil: } \\
\text { evaporation from bare soil }\end{array}$ \\
\hline$E_{\mathrm{ca}}$ & $\mathrm{L}^{3} \mathrm{~L}^{-2} \mathrm{~T}^{-1}$ & $\begin{array}{l}\text { canopy evaporation: } \\
\text { evaporation of the water } \\
\text { intercepted by canopy, } \\
\text { interception loss }\end{array}$ \\
\hline$E_{\mathrm{la}}$ & $\mathrm{L}^{3} \mathrm{~L}^{-2} \mathrm{~T}^{-1}$ & evaporation from lake \\
\hline$E_{\text {re }}$ & $\mathrm{L}^{3} \mathrm{~L}^{-2} \mathrm{~T}^{-1}$ & evaporation from reservoir \\
\hline$E_{\text {ri }}$ & $\mathrm{L}^{3} \mathrm{~L}^{-2} \mathrm{~T}^{-1}$ & evaporation from river \\
\hline$E_{\mathrm{sn}}$ & $\mathrm{L}^{3} \mathrm{~L}^{-2} \mathrm{~T}^{-1}$ & $\begin{array}{l}\text { sublimation: evaporation } \\
\text { from snow }\end{array}$ \\
\hline AET & $\mathrm{L}^{3} \mathrm{~L}^{-2} \mathrm{~T}^{-1}$ & $\begin{array}{l}\text { actual evapotranspiration: } \\
\text { total evapotranspiration }\end{array}$ \\
\hline
\end{tabular}

Definition

amount of water extracted, from surface water or groundwater, by humans for various economic sectors. It represents the sum of the water consumed by humans (water consumption), evaporative and percorative loss, and water returned to the groundwater or surface water, being the part of the water not consumed.

amount of water extracted by humans considering unlimited water supply.

amount of water extracted by humans considering water availability.

part of water extracted that evapotranspirates during use of purpose.

amount of water extracted by humans for households and small businesses.

amount of water extracted by humans to cool thermal and nuclear power plants, which are using the heat, obtain by burning of fossil fuels, gas, biomass or through nuclear energy, to produce electricity.

amount of water extracted by humans for use in agricultural irrigation.

amount of water extracted by humans for use in industrial sector, in some models this sector includes manufacturing and electricity sectors. amount of water extracted by humans for livestock production.

amount of water extracted by humans in factories, for producing goods.

part of water extracted by humans that returns into the soil, groundwater, lake, reservoir, river and ocean.

seawater or saline and brackish water extracted by humans to be used for various economic sectors, for example, for domestic and manufacturing sectors or for electricity production. amount of water that changes from liquid to vapor from the bare soil (not through vegetation).

amount of water accumulated on the vegetation that changes from liquid to vapor.

water that changes from liquid to vapor from a lake.

water that changes from liquid to vapor from a reservoir.

water that changes from liquid to vapor from a river.

water that changes from solid (snow and ice) to vapor.

total amount of water from transpiration, evaporation, interception losses, and sublimation, considering water availability. 


\begin{tabular}{|c|c|c|}
\hline PET & $\mathrm{L}^{3} \mathrm{~L}^{-2} \mathrm{~T}^{-1}$ & potential evapotranspiration \\
\hline$E_{\text {we }}$ & $\mathrm{L}^{3} \mathrm{~L}^{-2} \mathrm{~T}^{-1}$ & evaporation from wetland \\
\hline$M$ & $\mathrm{~L}^{3} \mathrm{~L}^{-2} \mathrm{~T}^{-1}$ & snowmelt \\
\hline$P_{\mathrm{de}}$ & $\mathrm{L}^{3} \mathrm{~L}^{-2} \mathrm{~T}^{-1}$ & dew \\
\hline$P_{\mathrm{dr}}$ & $\mathrm{L}^{3} \mathrm{~L}^{-2} \mathrm{~T}^{-1}$ & drip \\
\hline$P_{\mathrm{ra}}$ & $\mathrm{L}^{3} \mathrm{~L}^{-2} \mathrm{~T}^{-1}$ & rainfall \\
\hline$P_{\mathrm{sn}}$ & $\mathrm{L}^{3} \mathrm{~L}^{-2} \mathrm{~T}^{-1}$ & snowfall \\
\hline$P_{\mathrm{sf}}$ & $\mathrm{L}^{3} \mathrm{~L}^{-2} \mathrm{~T}^{-1}$ & stemflow \\
\hline$P_{\text {th }}$ & $\mathrm{L}^{3} \mathrm{~L}^{-2} \mathrm{~T}^{-1}$ & throughfall \\
\hline$P_{\text {tot }}$ & $\mathrm{L}^{3} \mathrm{~L}^{-2} \mathrm{~T}^{-1}$ & $\begin{array}{l}\text { total precipitation: } \\
\text { precipitation }\end{array}$ \\
\hline$Q_{\text {iu }}$ & $\mathrm{L}^{3} \mathrm{~T}^{-1}$ & $\begin{array}{l}\text { inflow from upstream } \\
\text { surface water bodies }\end{array}$ \\
\hline$Q_{\mathrm{ib}}$ & $\mathrm{L}^{3} \mathrm{~T}^{-1}$ & $\begin{array}{l}\text { inter-basin water transfer: } \\
\text { trans-basin diversion }\end{array}$ \\
\hline$Q_{\mathrm{la}}$ & $\mathrm{L}^{3} \mathrm{~T}^{-1}$ & outflow from lake \\
\hline$Q_{\mathrm{re}}$ & $\mathrm{L}^{3} \mathrm{~T}^{-1}$ & outflow from reservoir \\
\hline$Q_{\text {ri }}$ & $\mathrm{L}^{3} \mathrm{~T}^{-1}$ & $\begin{array}{l}\text { streamflow: outflow, flow, } \\
\text { river discharge }\end{array}$ \\
\hline $\begin{array}{l}Q_{\mathrm{we}} \\
R_{\mathrm{cr}}\end{array}$ & $\begin{array}{l}\mathrm{L}^{3} \mathrm{~T}^{-1} \\
\mathrm{~L}^{3} \mathrm{~L}^{-2} \mathrm{~T}^{-1}\end{array}$ & $\begin{array}{l}\text { outflow from wetland } \\
\text { capillary rise }\end{array}$ \\
\hline$R_{\mathrm{ct}}$ & $\mathrm{L}^{3} \mathrm{~L}^{-2} \mathrm{~T}^{-1}$ & channel transmission losses \\
\hline$R_{\mathrm{gw}}$ & $\mathrm{L}^{3} \mathrm{~L}^{-2} \mathrm{~T}^{-1}$ & groundwater runoff \\
\hline$R_{\mathrm{gwr}}$ & $\mathrm{L}^{3} \mathrm{~L}^{-2} \mathrm{~T}^{-1}$ & groundwater recharge \\
\hline$R_{\text {ho }}$ & $\mathrm{L}^{3} \mathrm{~L}^{-2} \mathrm{~T}^{-1}$ & $\begin{array}{l}\text { Hortonian overland flow: } \\
\text { infiltration excess overland } \\
\text { flow, flooding excess } \\
\text { overland flow, unsaturated } \\
\text { overland flow } \\
\text { infiltration }\end{array}$ \\
\hline$R_{\text {if }}$ & $\mathrm{L}^{3} \mathrm{~L}^{-2} \mathrm{~T}^{-1}$ & $\begin{array}{l}\text { interflow: subsurface storm } \\
\text { flow, subsurface runoff }\end{array}$ \\
\hline$R_{\mathrm{pe}}$ & $\mathrm{L}^{3} \mathrm{~L}^{-2} \mathrm{~T}^{-1}$ & percolation \\
\hline
\end{tabular}

total amount of water from transpiration, evaporation, interception losses, and sublimation, considering unlimited water source.

water which changes from liquid to vapor above a wetland.

water that changes from solid to liquid.

atmospheric water condensing directly on the land surface

water spill from canopy to the ground when the water exceeds the canopy interception capacity (through dripping).

liquid precipitation that falls in a given area and in a given time, provided as climate input data in ISIMIP2.

solid precipitation, combined in small ice crystals, that falls in a given area and in a given time, provided as climate input data in ISIMIP2.

water spill to the ground through canopy, which flows along twigs, branches and stems.

water spill to the ground through canopy spaces.

liquid or solid water resulting from the condensation or freezing of water vapor and falling to the ground under gravity.

water from upstream, which can be a river, a lake, a wetland, a reservoir.

anthropogenic transport of water from one river basin, where it is available, to another basin where water is less available or could be utilized for other purposes. This could be an output at a location and input to another. water that flows out of a lake to a river, wetland or reservoir. water that flows out of a reservoir, a lake which is made by humans, to a river.

volumetric flow rate of water through a river cross-section. The streamflow is transfer through a channel to the ocean or to an inland sink.

water that flows out of a wetland to a river.

water rising from roundwater to soil under the influence of capillary forces.

water from river storage which recharges the groundwater storage.

water that leaves the groundwater storage to a river, lake or wetland.

water leaving the last soil layer(s) and reaching the groundwater storage. In some models, this variable describes seepage.

water that runs off over the soil surface because the rainfall intensity exceeds the infiltration capacity.

water from rainfall or throughfall or snowmelt or irrigation which flows through the soil surface into the root zone, under the effect of gravity.

water that runs-off laterally from the soil.

amount of water that penetrates in the soil layers, beyond the root zone of plants 


$\begin{array}{ccl}R_{\text {sat }} & \mathrm{L}^{3} \mathrm{~L}^{-2} \mathrm{~T}^{-1} & \text { saturation excess overland } \\ & & \text { flow: saturation overland } \\ & & \text { flow, saturation excess } \\ & & \text { runoff, Dunne runoff } \\ & & \text { surface runoff: overland } \\ & & \text { flow, fast runoff, flood } \\ & & \text { runoff, surface flow, } \\ & \mathrm{L}^{3} \mathrm{~L}^{-2} \mathrm{~T}^{-1} & \\ & & \\ & & \text { surface or direct runoff } \\ & & \text { total runoff }\end{array}$

$\begin{array}{ccl}S_{\text {ca }} & \mathrm{L}^{3} \mathrm{~L}^{-2} & \text { canopy storage } \\ S_{\mathrm{gl}} & \mathrm{L}^{3} \mathrm{~L}^{-2} & \text { glacier storage } \\ S_{\mathrm{gw}} & \mathrm{L}^{3} \mathrm{~L}^{-2} & \text { groundwater storage }\end{array}$

$S_{\text {la }} \quad \mathrm{L}^{3} \mathrm{~L}^{-2} \quad$ lake storage

$\begin{array}{lll}S_{\text {So }} & \mathrm{L}^{3} \mathrm{~L}^{-2} & \text { soil storage } \\ S_{\text {re }} & \mathrm{L}^{3} \mathrm{~L}^{-2} & \text { reservoir storage }\end{array}$

$S_{\text {ri }} \quad \mathrm{L}^{3} \mathrm{~L}^{-2} \quad$ river storage

$\begin{array}{lll}S_{\text {sn }} & \mathrm{L}^{3} \mathrm{~L}^{-2} & \text { snow storage } \\ S_{\text {soc }} & \mathrm{L}^{3} \mathrm{~L}^{-2} & \text { snow held on the canopy } \\ S_{\text {suc }} & \mathrm{L}^{3} \mathrm{~L}^{-2} & \text { snow under the canopy } \\ S_{\text {sw }} & \mathrm{L}^{3} \mathrm{~L}^{-2} & \begin{array}{l}\text { surface water bodies: } \\ \text { surface water }\end{array} \\ S_{\text {we }} & \mathrm{L}^{3} \mathrm{~L}^{-2} & \text { wetland storage }\end{array}$

$T \quad \mathrm{~L}^{3} \mathrm{~L}^{-2} \mathrm{~T}^{-1} \quad$ transpiration water that runs off over the soil surface because the soil is saturated.

water that runs-off over the soil surface as Hortonian overland flow and / or saturation excess overland flow.

total amount of water that runs-off the grid-cell, either over the soil surface, or from the subsurface (lateral flow). In some studies, the streamflow is converted to runoff by dividing the streamflow values with the area upstream of the gauging station (for example, the area upstream of station according to the DDM30' river network Döll and Lehner, 2002). compartment that retains water from precipitation and loses water through throughfall, stemflow and interception loss (evaporation). compartment which retains water from rainfall, snowfall, and loses water through sublimation, glacier melt, runoff from liquid precipitation.

compartment, beneath the soil water compartment, that receives water from seepage, groundwater recharge, and loses water through capillary rise, groundwater runoff, and abstraction for human water use. Hydrologically, it includes saturated zone or phreatic zone.

compartment that fills with water through fluxes above and beyond the ground and stores water for a residence time. It loses water through discharge to other storages, evaporation, groundwater recharge, and water abstraction for human water use.

compartment that keeps and loses water from flows above and beyond the ground's surface. Hydrologically, it includes unsaturated zone. compartment that fills with water behind dams through fluxes above and beyond the ground and stores water for a residence time. It loses water through discharge to other storages, evaporation, groundwater recharge, and water abstraction for human water use.

compartment filled with water through fluxes above and beyond the ground. It loses water through river discharge, evaporation, channel transmission losses and water abstraction for human water use.

compartment that accumulates snow below freezing temperature and loses snow by melting and sublimation.

snow compartment that accumulates snow on the vegetation.

amount of snow accumulated under the canopy or on the soil

surface water bodies can include river, lake, wetland, and reservoir.

compartment, as a transition area between the terrestrial and aquatic systems, filled by precipitation or inflow and emptied by evapo(transpi)ration, outflow and groundwater recharge. water evaporated by plants through their stomata. 
Table S85. Downscaled and bias-adjusted output from CMIP5 Global Climate Models (GCMs) used by the ISIMIP2b Impact Models $^{1}$ with a spatial resolution of $0.5^{\circ} \times 0^{\circ}$ and a daily temporal resolution

\begin{tabular}{|c|c|}
\hline GCM output used $^{2}$ & Models \\
\hline IPSL-CM5A-LR & $\begin{array}{l}\text { CLM4.5, CLM5.0, CWatM, DBH, H08, JULES-W1, LPJmL, MATSIRO, mHM, MPI-HM, ORCHIDEE, PCR- } \\
\text { GLOBWB, WaterGAP2, WAYS }\end{array}$ \\
\hline HadGEM2-ES & $\begin{array}{l}\text { CLM4.5, CLM5.0, CWatM, H08, JULES-W1, LPJmL, MATSIRO, mHM, ORCHIDEE, PCR-GLOBWB, WaterGAP2, } \\
\text { WAYS }\end{array}$ \\
\hline GFDL-ESM2M & $\begin{array}{l}\text { CLM4.5, CLM5.0, CWatM, H08, JULES-W1, LPJmL, MATSIRO, mHM, MPI-HM, ORCHIDEE, ORCHIDEE- } \\
\text { DGVM, PCR-GLOBWB, WaterGAP2, WAYS }\end{array}$ \\
\hline MIROC5 & $\begin{array}{l}\text { CLM4.5, CLM5.0, CWatM, H08, JULES-W1, LPJmL, MATSIRO, mHM, MPI-HM, ORCHIDEE, PCR-GLOBWB, } \\
\text { WaterGAP2, WAYS }\end{array}$ \\
\hline
\end{tabular}

Notes:

1. Source data: https://www.isimip.org/gettingstarted/availability-input-data-isimip2b/, 2. EartH2Observe, WFDEI and ERA-Interim data Merged and Biascorrected for ISIMIP (EWEMBI), http://dataservices.gfz-potsdam.de/pik/showshort.php?id=escidoc:1809891. Data source of the bias-corrected atmospheric data is ISIMIP project.

2. Data source of the original data: CMIP5 (Coupled Model Intercomparison Project Phase 5)

Reference: Lange, S. (2016): EartH2Observe, WFDEI and ERA-Interim data Merged and Bias-corrected for ISIMIP (EWEMBI). GFZ Data Services. http://doi.org/10.5880/pik.2016.004 
Table S86. Climate variables used as input for the ISIMIP2b Impact Models ${ }^{1}$ with a spatial resolution of $0.5^{\circ} \times 0.5^{\circ}$ and a time span between 1661 - 2299

\begin{tabular}{|c|c|c|c|}
\hline Variable name & Symbol (unit) & $\begin{array}{l}\text { Temporal } \\
\text { resolution }\end{array}$ & Models \\
\hline snowfall & $\operatorname{prsn}\left(\mathrm{kg} \mathrm{m}^{-2} \mathrm{~s}^{-1}\right)$ & $\begin{array}{l}3 \text { hourly } \\
\text { daily }\end{array}$ & $\begin{array}{l}\text { MATSIRO } \\
\text { H08 }\end{array}$ \\
\hline total precipitation & $\operatorname{pr}\left(\mathrm{kg} \mathrm{m}^{-2} \mathrm{~s}^{-1}\right)$ & daily & $\begin{array}{l}\text { CLM4.5, CLM5.0, DBH, JULES-W1, H08, LPJmL, Mac- } \\
\text { PDM.20, mHM, MPI-HM, ORCHIDEE, PCR-GLOBWB, } \\
\text { WaterGAP2 }\end{array}$ \\
\hline near surface wind speed & sfcWind $\left(\mathrm{m} \mathrm{s}^{-1}\right)$ & daily & $\begin{array}{l}\text { CLM4.5, CWatM,DBH, H08, JULES-W1, Mac-PDM.20, } \\
\text { ORCHIDEE }\end{array}$ \\
\hline eastward wind & ua $\left(\mathrm{m} \mathrm{s}^{-1}\right)$ & daily & CLM5.0, ORCHIDEE \\
\hline westward wind & $\mathrm{va}\left(\mathrm{m} \mathrm{s}^{-1}\right)$ & daily & CLM5.0, ORCHIDEE \\
\hline surface air pressure & ps $(\mathrm{Pa})$ & $\begin{array}{l}\text { daily } \\
3 \text { hourly }\end{array}$ & $\begin{array}{l}\text { CLM5.0, CWatM, DBH, H08, JULES-W1, ORCHIDEE } \\
\text { MATSIRO, ORCHIDEE }\end{array}$ \\
\hline near surface specific humidity & huss $\left(\mathrm{kg} \mathrm{kg}^{-1}\right)$ & daily & CLM4.5, CWatM, H08, JULES-W1, H08 \\
\hline relative humidity & rhs $(\%)$ & $\begin{array}{l}\text { daily } \\
3 \text { hourly }\end{array}$ & $\begin{array}{l}\text { CLM5.0, CWatM, DBH, JULES-W1, Mac-PDM. } 20 \\
\text { MATSIRO }\end{array}$ \\
\hline mean temperature & $\operatorname{tas}(\mathrm{K})$ & 3 hourly & $\begin{array}{l}\text { DBH, CWatM,JULES-W1, H08, LPJmL, Mac-PDM.20, } \\
\text { mHM, MPI-HM, PCR-GLOBWB, WaterGAP2, CLM4.5, } \\
\text { CLM5.0, JULES-W1, PCR-GLOBWB, } \\
\text { MATSIRO }\end{array}$ \\
\hline maximum temperature & $\operatorname{tasmax}(\mathrm{K})$ & daily & $\begin{array}{l}\text { DBH, CWatM, JULES-W1, mHM, ORCHIDEE , PCR- } \\
\text { GLOBWB }\end{array}$ \\
\hline minimum temperature & $\operatorname{tasmin}(\mathrm{K})$ & daily & $\begin{array}{l}\text { DBH, CWatM, JULES-W1, mHM, ORCHIDEE, PCR- } \\
\text { GLOBWB }\end{array}$ \\
\hline $\begin{array}{l}\text { shortwave downwelling } \\
\text { radiation }\end{array}$ & $\operatorname{rsds}\left(\mathrm{W} \mathrm{m}^{-2}\right)$ & daily & $\begin{array}{l}\text { CLM4.5, CLM5.0, CWatM, DBH, JULES-W1, H08, } \\
\text { LPJmL }^{2} \text {, Mac-PDM.20, ORCHIDEE, WaterGAP2 }\end{array}$ \\
\hline longwave downwelling radiation & rlds $\left(\mathrm{W} \mathrm{m}^{-2}\right)$ & $\begin{array}{l}3 \text { hourly } \\
\text { daily }\end{array}$ & $\begin{array}{l}\text { MATSIRO } \\
\text { CLM4.5, CLM5.0, CWatM, DBH, JULES-W1, H08, Mac- } \\
\text { PDM.20, ORCHIDEE, WaterGAP2 }\end{array}$ \\
\hline & & 3 hourly & MATSIRO \\
\hline
\end{tabular}

\section{Note:}

1: Data source of the bias-corrected atmospheric data: ISIMIP project and Lange, 2016; Data source of the original data: CMIP5 (Coupled Model Intercomparison Project Phase 5)

2: LPJmL: Long wave net radiation derived from longwave downwelling radiation and mean temperature.

Reference: Lange, S. (2016): EartH2Observe, WFDEI and ERA-Interim data Merged and Bias-corrected for ISIMIP (EWEMBI). GFZ Data Services. http://doi.org/10.5880/pik.2016.004 
Table S87. $\mathrm{CO}_{2}$ concentrations and Land use datasets

\begin{tabular}{|c|c|c|c|c|c|c|c|}
\hline Dataset & $\begin{array}{l}\text { Variable name / short } \\
\text { description }\end{array}$ & Symbol & Data source & Time Span & $\begin{array}{l}\text { Spatial } \\
\text { Resolutio } \\
\mathrm{n}\end{array}$ & $\begin{array}{l}\text { Temporal } \\
\text { Resolution }\end{array}$ & Models \\
\hline $\begin{array}{l}\text { Atmospheric } \\
\mathrm{CO}_{2} \\
\text { concentrations }\end{array}$ & $\begin{array}{l}\mathrm{CO}_{2} \text { concentrations / } \\
\text { Values are constant } \\
\text { for } 1661-1860, \\
\text { following } \\
\text { observations from } \\
1861-2005 \text {, and } \\
\text { correspond to RCP } \\
2.6 \text { and } 6.0 \text { from } \\
2006-2299\end{array}$ & $\mathrm{CO}_{2}$ & & $\begin{array}{l}1661- \\
2299\end{array}$ & $0.5^{\circ} \times 0.5^{\circ}$ & constant & $\begin{array}{l}\text { CLM4.5, CLM5.0, } \\
\text { DBH, JULES-W1. } \\
\text { LPJmL, } \\
\text { ORCHIDEE, } \\
\text { PCR-GLOBWB } \\
\text { H08 }\end{array}$ \\
\hline MIRCA2000 & $\begin{array}{l}\text { irrigated and rainfed } \\
\text { crop areas / around } \\
\text { the year } 2000\end{array}$ & $\mathrm{LU}$ & $\begin{array}{l}\text { Portmann et } \\
\text { al., } 2000\end{array}$ & $\begin{array}{l}1998- \\
2002\end{array}$ & $\begin{array}{l}0.5^{\circ} \times 0.5^{\circ} \\
/ \\
5^{\prime} \times 5^{\prime}\end{array}$ & monthly & $\begin{array}{l}\text { H08, ORCHIDEE, } \\
\text { MPI-HM }\end{array}$ \\
\hline $\begin{array}{l}\text { Historical, } \\
\text { gridded land } \\
\text { use }\end{array}$ & $\begin{array}{l}\text { Land use / } 5 \text { Rainfed } \\
\text { crop land, irrigated } \\
\text { crop land, pastures } \\
\text { and total crop land } \\
\text { (the sum of rainfed } \\
\text { and irrigated) }\end{array}$ & LUH & $\begin{array}{l}\text { HYDE 3.2, } \\
\text { Klein } \\
\text { Goldewijk, } \\
2017\end{array}$ & $\begin{array}{l}10000 \\
\mathrm{BCE}- \\
2015 \mathrm{CE}\end{array}$ & $0.5^{\circ} \times 0.5^{\circ}$ & $\begin{array}{l}2000-2015 \\
\text { CE: annual } \\
1700-2000 \\
\text { CE: } 10 \text { years }\end{array}$ & $\begin{array}{l}\text { CLM4.5, CLM5.0, } \\
\text { CWatM, } \\
\text { H08LPJmL, } \\
\text { mHM, MPI-HM, } \\
\text { ORCHIDEE, } \\
\text { PCR-GLOBWB }\end{array}$ \\
\hline $\begin{array}{l}\text { Future land- } \\
\text { use patterns }\end{array}$ & $\begin{array}{l}\text { Land use and land } \\
\text { cover / } 6 \text { land-use } \\
\text { types: rain-fed crop } \\
\text { land, irrigated crop- } \\
\text { land, rain-fed } \\
\text { bioenergy, irrigated } \\
\text { bio-energy, pastures, } \\
\text { natural vegetation } \\
\text { and urban areas }\end{array}$ & LULC & $\begin{array}{l}\text { MAgPIE } \\
\text { land-use } \\
\text { model } \\
\text { according to } \\
\text { the SSP2 } \\
\text { shared-socio- } \\
\text { economic } \\
\text { pathway and } \\
\text { RCP 2.6 / } \\
\text { RCP 6.0. }\end{array}$ & $\begin{array}{l}2006- \\
2100\end{array}$ & $0.5^{\circ} \times 0.5^{\circ}$ & annual & $\begin{array}{l}\text { JULES-W1, MPI- } \\
\text { HM }\end{array}$ \\
\hline
\end{tabular}

Note: 1. https://www.isimip.org/gettingstarted/details/30/

Reference:

1. Klein Goldewijk, Dr. ir. C.G.M. (Utrecht University) (2017): Anthropogenic land-use estimates for the Holocene; HYDE 3.2. DANS. https://doi.org/10.17026/dans-25g-gez3

2. Portmann, F. T., Siebert, S. \& Döll, P. (2010): MIRCA2000 - Global monthly irrigated and rainfed crop areas around the year 2000: A new high-resolution data set for agricultural and hydrological modeling, Global Biogeochemical Cycles, 24, GB 1011, doi:10.1029/2008GB003435. 
Table S88. Other input date sets used by the ISIMIP2b Impact models

\begin{tabular}{|c|c|c|c|c|}
\hline Dataset & Data source & Spatial Resolution & $\begin{array}{l}\text { Temporal } \\
\text { resolution } \\
\text { / details / } \\
\text { Time span }\end{array}$ & Models \\
\hline soil type $^{1}$ & ISIMIP2b & $0.5^{\circ} \times 05^{\circ}$ & static & $\begin{array}{l}\text { CLM4.5, CLM5.0, DBH, JULES-W1, LPJmL, } \\
\text { MPI-HM, ORCHIDEE, PCR-GLOBWB, } \\
\text { WaterGAP2 }^{4}\end{array}$ \\
\hline $\begin{array}{l}\text { plant functional types (PFT) } \\
\text { parameters }\end{array}$ & $\begin{array}{l}\text { Samanta et. al., } \\
2014\end{array}$ & $0.5^{\circ} \times 05^{\circ}$ & static & JULES-W1 \\
\hline $\begin{array}{l}\text { topographical information } \\
\text { (subgrid slope distribution) }\end{array}$ & $\begin{array}{l}\text { Hagemann and } \\
\text { Gates, } 2003\end{array}$ & $1 \mathrm{~km} \times 1 \mathrm{~km}$ & static & MPI-HM \\
\hline River-routing network & $\begin{array}{l}\text { DDM } 30^{2} \text { (Döll and } \\
\text { Lehner, 2002) } \\
\text { TRIP model } \\
\text { Simulated } \\
\text { Topological } \\
\text { Network (STN- } \\
\text { 30p) (Vörösmarty } \\
\text { et al., 2000) } \\
\text { HydroSHEDS } \\
\text { et al., 2006 }\end{array}$ & $30^{\prime} \times 30^{‘}\left(0.5^{\circ} \times 05^{\circ}\right)$ & static & $\begin{array}{l}\text { CLM4.5, CLM5.0, CWatM, H08, LPJmL, MPI- } \\
\text { HM, PCR-GLOBWB }{ }^{5} \text {, WaterGAP2 } \\
\text { MATSIRO } \\
\text { ORCHIDEE }\end{array}$ \\
\hline crop parameters & & $0.5^{\circ} \times 05^{\circ}$ & static & $\mathrm{H} 08$ \\
\hline Albedo & 2012 & $0.5^{\circ} \times 05^{\circ}$ & static & H08, CWatM ${ }^{6}$ \\
\hline Land-sea mask & ISIMIP2b & $0.5^{\circ} \times 05^{\circ}$ & static & $\begin{array}{l}\text { CLM5.0, DBH, CWatM, Mac-PDM.20, mHM, } \\
\text { MPI-HM, ORCHIDEE, PCR-GLOBWB, } \\
\text { WaterGAP2 }\end{array}$ \\
\hline GRanD & Lehner et al., 2011 & $\begin{array}{l}6862 \text { dams and their associated } \\
\text { reservoirs, total storage } \\
\text { capacity of } 6197 \mathrm{~km}^{3}\end{array}$ & static & $\begin{array}{l}\text { CWatM }^{7}, \text { H08, LPJmL, MATSIRO, PCR- } \\
\text { GLOBWB, WaterGAP2 }{ }^{8}\end{array}$ \\
\hline $\begin{array}{l}\text { Water abstraction for domestic } \\
\text { and industrial uses consistent } \\
\text { with SSP2 and RCP } 6.0\end{array}$ & $\begin{array}{l}\text { ISIMIP } 2 \mathrm{~b}^{3}, \text { multi- } \\
\text { model averages of } \\
\text { PCR, WaterGAP, } \\
\text { H08 }\end{array}$ & $0.5^{\circ} \times 05^{\circ}$ & $\begin{array}{l}\text { available } \\
\text { until } \\
2050, \text { the } \\
\text { values are } \\
\text { kept static } \\
\text { from } 2050 \\
\text { onwards }\end{array}$ & LPJmL \\
\hline $\begin{array}{l}\text { Global Lakes and Wetlands } \\
\text { Database (GLWD) }\end{array}$ & $\begin{array}{l}\text { Lehner and Döll, } \\
\text { 2004; Lehner et } \\
\text { al., } 2011\end{array}$ & $1: 1$ to $1: 3$ million resolution & static & MPI-HM, PCR-GLOBWB, WaterGAP2 \\
\hline $\begin{array}{l}\text { Regarding soil data — we use the } \\
\text { SoilGrids } 250 \text { from ISRIC: }\end{array}$ & Hengl et al., 2017 & $0.002^{\circ} \times 0.002^{\circ}$ & static & $\mathrm{mHM}$ \\
\hline
\end{tabular}

Note: 1. H08 does not require soil type. In addition to estimate subgrid slope distribution, information about permafrost, slope, geology etc. are required (see Hanasaki et al., 2018). 2. DDM30 = the 30' global drainage direction map (Döll and Lehner, 2002). 3. For modelling groups that do not have their own representation, ISIMIP2b provides files containing the multi-model mean from WaterGAP2, PCR-GLOBWB and H08 scenarios for domestic and industrial uses under SSP2 from the Water Futures and Solutions Project (WFaS; Wada et al., 2016). 4. Soil data from WISE Available Water Capacity (Batjes, 2012). 5. PCR-GLOBWB combines DDM30 with GLWD. 6. Muller et al., 2012. 7. CWatm: HydroLakes database (Messager et al., 2016; Lehner et al., 2011) 8. WaterGAP2 uses a pre-published and adjusted version of GRanD, see https://www.arcgis.com/home/item.html?id=d966db9c7b2949ac8380458d7020adf9.

\section{References:}

Batjes, N. H. (2012): ISRIC-WISE derived soil properties on 5 by 5 arc-minutes global grid (ver. 1.2). ISRIC World Soil Information, ISRIC Report 2012 / 01. https://www.isric.org/sites/default/files/isric report 2012 01.pdf

Döll, P., Lehner, B. (2002): Validation of a new global 30-minute drainage direction map. Journal of Hydrology, 258(1-4), $214-231$.

Flörke, M., Kynast, E., Bärlund, I., Eisner, S., Wimmer, F., Alcamo, J. (2013): Domestic and industrial water uses of the past 60 years as a mirror of socioeconomic development: A global simulation study. Global Environ. Change, 23, 144-156. doi:10.1016/j.gloenvcha.2012.10.018.

Hagemann, S., Gates, L. D. (2003): Improving a subgrid runoff parameterization scheme for climate models by the use of high resolution data derived from satellite observations. Climate Dynamics, Volume 21, Issue 3-4, 349-359.

Hanasaki, N., Yoshikawa, S., Pokhrel, Y., Kanae, S. (2018): A global hydrological simulation to specify the sources of water used by humans. Hydrol. Earth Syst. Sci., 22, 789-817, https://doi.org/10.5194/hess-22-789-2018. 
Hengl, T., de Jesus, J.M., Heuvelink, G.B., Gonzalez, M.R., Kilibarda, M., Blagotić, A., Shangguan, W., Wright, M.N., Geng, X., Bauer-Marschallinger, B. and Guevara, M.A., 2017. SoilGrids250m: Global gridded soil information based on machine learning. PLoS one, 12(2).

Lehner, B. and Döll, P. (2004): Development and validation of a global database of lakes, reservoirs and wetlands. Journal of Hydrology 296, 1-4: 1-22

Lehner, B., Verdin, K., and Jarvis, A.: HydroSHEDS Technical Documentation, World Wildlife Fund US, Washington, DC., 26,

http://hydrosheds.cr.usgs.gov., 2006.

Lehner, B., Reidy Liermann, C. Revenga, C., Vörösmarty, C., Fekete, B., Crouzet, P., Döll, P., Endejan, M., Frenken, K., Magome, J., Nilsson, C., Robertson, J.C., Rodel, R., Sindorf, N., Wisser, D. (2011): High-Resolution Mapping of the World's Reservoirs and Dams for Sustainable River-Flow Management. Frontiers in Ecology and the Environment 9 (9), 494-502. http://dx.doi.org/10.1890/100125.

Samanta, A., Ganguly, S., Schull, M. A., Shabanov, N. V., Knyazikhin, Y., and Myneni, R. B.: Collection 5 MODIS LAI/FPAR Products, Presented at AGU Fall Meeting, San Francisco, USA, 15-19 December, 2008, 2012.

Vörösmarty, C. J., Fekete, B. M., Meybeck, M., and Lammers, R. B.: Global system of rivers: Its role in organizing continental land mass and defining land-To-Ocean linkages, Global Biogeochem. Cy., 14, 599-621, https://doi.org/10.1029/1999GB900092, 2000.

Messager, M. L., Lehner, B., Grill, G., Nedeva, I., and Schmitt, O.: Estimating the volume and age of water stored in global lakes using a geo-statistical approach, 7, 13603, 10.1038/ncomms13603, 2016

Muller, P. J., Lewis, P., Fischer, J., North, P., and Framer, U.: The ESA GlobAlbedo Project for mapping the Earth's land 1105 surface albedo for 15 Years from European Sensors., paper presented at IEEE Geoscience and Remote Sensing Symposium (IGARSS) IEEE Geoscience and Remote Sensing Symposium (IGARSS) 2012, Munich, Germany, 22-27.7.12., 2012

Wada, Y., Flörke, M., Hanasaki, N., Eisner, S., Fischer, G., Tramberend, S., Satoh, Y., van Vliet, M. T. H., Yillia, P., Ringler, C., Burek, P., and Wiberg, D. (2016): Modeling global water use for the 21st century: the Water Futures and Solutions (WFaS) initiative and its approaches. Geosci. Model Dev., 9, 175222, https://doi.org/10.5194/gmd-9-175-2016. 
Table S89. Natural Vegetation datasets used by the ISIMIP2b impact models

\begin{tabular}{|c|c|c|c|c|}
\hline Dataset & Data source & Time Span & Spatial Resolution & Models \\
\hline $\begin{array}{l}\text { Land Surface Parameter set } 2 \\
\text { (LSP2) }\end{array}$ & Hagemann, 2002 & 2001 & $0.5^{\circ} \times 0.5^{\circ}$ & LPJmL, MPI-HM \\
\hline MODIS & NASA & & $0.5^{\circ} \times 0.5^{\circ}$ & CLM5.0, WaterGAP2 ${ }^{1}$ \\
\hline $\begin{array}{l}\text { Global Land Cover Map } \\
\text { (GlobCover) }\end{array}$ & $\begin{array}{l}\text { European Space } \\
\text { Agency GlobCover } \\
\text { Portal (ESA) }\end{array}$ & 2009 & $0.5^{\circ} \times 0.5^{\circ}$ & $\begin{array}{l}\text { Mac-PDM.20, mHM, } \\
\text { PCR-GLOBWB }\end{array}$ \\
\hline
\end{tabular}

Note: 1. WaterGAP2 uses The International Geosphere-Biosphere Programme (IGBP) classification based on MODIS data for the year 2004.

References:

Hagemann, S (2002). An Improved Land Surface Parameter Dataset for Global and Regional Climate Models. Max-Planck-Institut für Meteorologie. MPI Report 336, ISSN 0937-1060.

https://modis.gsfc.nasa.gov/data/

http://www.fao.org/land-water/land/land-governance/land-resources-planning-toolbox/category/details/en/c/1036354/

http://due.esrin.esa.int/page globcover.php 
Table S90. Socio-economic datasets with a spatial resolution of $0.5^{\circ} \times 0.5^{\circ}$ and $5^{\prime} \times 5^{\circ}$ and an annual temporal resolution

\begin{tabular}{|c|c|c|c|c|c|}
\hline Dataset name & Variable name / details & Symbol (unit) & Data source & Time Span & Models \\
\hline $\begin{array}{l}\text { Historical, country-level } \\
\text { population }\end{array}$ & population & $\begin{array}{l}\text { pop (number } \\
\text { people) }\end{array}$ & $\begin{array}{l}\text { Hyde } 3.2 \\
\text { (Klein } \\
\text { Goldewijk et } \\
\text { al., 2010; } \\
\text { Klein } \\
\text { Goldewijk, } \\
\text { 2011) }\end{array}$ & $1861-2009$ & $\begin{array}{l}\text { PCR-GLOBWB } \\
\text { WaterGAP2 }\end{array}$ \\
\hline $\begin{array}{l}\text { Historical, gridded } \\
\text { population }\end{array}$ & population & $\begin{array}{l}\text { pop (number } \\
\text { people) }\end{array}$ & $\begin{array}{l}\text { Hyde } 3.2 \\
\text { (Klein } \\
\text { Goldewijk et } \\
\text { al., 2010; } \\
\text { Klein } \\
\text { Goldewijk, } \\
\text { 2011) }\end{array}$ & $1861-2005$ & $\begin{array}{l}\text { PCR-GLOBWB } \\
\text { WaterGAP2 }\end{array}$ \\
\hline $\begin{array}{l}\text { Future, country-level } \\
\text { population }\end{array}$ & $\begin{array}{l}\text { country-level population, } \\
\text { urban population and age } \\
\text { structure (in five-year } \\
\text { age bands) / based on } \\
\text { SSP2 }\end{array}$ & $\begin{array}{l}\text { pop (number } \\
\text { people) }\end{array}$ & $\begin{array}{l}\text { IIASA } \\
\text { (population \& } \\
\text { age structure) } \\
\text { and NCAR } \\
\text { (urbanshare) }\end{array}$ & $2006-2099$ & $\begin{array}{l}\text { PCR-GLOBWB } \\
\text { WaterGAP2 }\end{array}$ \\
\hline Future, gridded population & $\begin{array}{l}\text { population / based on } \\
\text { SSP2 } \\
\text { population / based on all } \\
\text { SSPs }\end{array}$ & $\begin{array}{l}\text { pop (number } \\
\text { people) }\end{array}$ & $\begin{array}{l}\text { Samir and } \\
\text { Lutz, 2014 } \\
\text { Jones and } \\
\text { O'Neill ,2016 }\end{array}$ & $\begin{array}{l}2006-2099 \\
2006-2100\end{array}$ & $\begin{array}{l}\text { PCR-GLOBWB } \\
\text { WaterGAP2 }\end{array}$ \\
\hline $\begin{array}{l}\text { Historical, country-level } \\
\text { Gross Domestic Product } \\
(\text { GDP })^{1}\end{array}$ & Gross Domestic Product & $\begin{array}{l}\text { GDP (per capita } \\
\text { and PPP \$) }\end{array}$ & $\begin{array}{l}\text { Geiger and } \\
\text { Frieler, } 2018\end{array}$ & $1861-2005$ & $\begin{array}{l}\text { PCR-GLOBWB } \\
\text { WaterGAP2 }\end{array}$ \\
\hline $\begin{array}{l}\text { Future, gridded Gross } \\
\text { Domestic Product (GDP) }\end{array}$ & Gross Domestic Product & $\begin{array}{l}\text { GDP (per capita } \\
\text { and PPP \$) }\end{array}$ & $\begin{array}{l}\text { Murakamiaan } \\
\text { and } \\
\text { Yamagata, } \\
2016\end{array}$ & 2006-2099 & $\begin{array}{l}\text { PCR-GLOBWB } \\
\text { WaterGAP2 }\end{array}$ \\
\hline $\begin{array}{l}\text { Future, country-level } \\
\text { Gross Domestic Product } \\
\text { (GDP) }\end{array}$ & Gross Domestic Product & $\begin{array}{l}\text { GDP (per capita } \\
\text { and PPP \$) }\end{array}$ & $\begin{array}{l}\text { Geiger and } \\
\text { Frieler, 2017; } \\
\text { Geiger and } \\
\text { Frieler, 2018 }\end{array}$ & $2006-2100$ & $\begin{array}{l}\text { PCR-GLOBWB } \\
\text { WaterGAP2 }\end{array}$ \\
\hline
\end{tabular}

Note: 1. National income (GDP / capita) and GDP time series (2005 PPP \$, purchasing power parities (PPP) conversion factor, local currency unit to international dollar) from 1850-2009 from Penn World Tables 8.1 extrapolated with per capita growth rates from the Maddison project, and extended by Penn World Tables 9.0 and World Development Indicators. Interpolated between 2006-2009 to match with OECD SSP2 projections starting in 2010. 2. All dataset: 1980 - 2100, by 10 years. The data in 1980-2010 are estimated by downscaling actual populations and GDPs by country, while those in 2020-2100 are estimated by downscaling projected populations and GDPs under three shared socioeconomic pathways (SSP): SSP1; SSP2; and SSP3, by country. 3. https://www.isimip.org/gettingstarted/details/32/

References:

1. Geiger, T., Frieler, K. (2017): Continuous national Gross Domestic Product (GDP) time series for 195 countries: past observations (1850-2005) harmonized with future projections according the Shared Socio-economic Pathways (2006-2100). GFZ Data Services. http://doi.org/10.5880/pik.2017.003 2. Geiger, T., Frieler, K. (2018): Continuous national Gross Domestic Product (GDP) time series for 195 countries: past observations (1850-2005) harmonized with future projections according the Shared Socio-economic Pathways (2006-2100) (Version 2.0) [Data set]. GFZ Data Services. https://doi.org/10.5880/pik.2018.010

3. Jones, B., O’Neill, B. C. (2016): Spatially explicit global population scenarios consistent with the Shared Socioeconomic Pathways. Environmental Research Letters, 11(8), 084003. http://doi.org/10.1088/1748-9326/11/8/084003

4. Klein Goldewijk, K., Beusen, A., Janssen, P. (2010): Long-term dynamic modeling of global population and built-up area in a spatially explicit way: HYDE 3.1. Holocene, 20(4), pp. 565-573, doi: http://dx.doi.org/10.1177/0959683609356587.

5. Klein Goldewijk, K., Beusen, A., Van Drecht, G., De Vos, M. (2011): The HYDE 3.1 spatially explicit database of human-induced global land-use change over the past 12,000 years. Global Ecology and Biogeography, 20(1), pp. 73-86, doi: http://dx.doi.org/10.1111/j.1466-8238.2010.00587.x. 6. Murakami, D., Yamagata, Y. (2016): Estimation of gridded population and GDP scenarios with spatially explicit statistical downscaling. ArXiv, 1610.09041, URL: https://arxiv.org/abs/1610.09041. 
Table S91. Hydrological output data of the ISMIP2b global water models1 with a spatial resolution of $0.5^{\circ} \times 0.5^{\circ}$

\begin{tabular}{|c|c|c|}
\hline Hydrological variable & $\begin{array}{l}\text { ISIMIP2b protocol Symbol } \\
\text { (Units) / Temporal resolution }\end{array}$ & Models \\
\hline Runoff & qtot $\left(\mathrm{kg} \mathrm{m}^{-2} \mathrm{~s}^{-1}\right) /$ daily & $\begin{array}{l}\text { CLM4.5, CLM5.0, CWatM, DBH, H08, JULES-W1, LPJmL, Mac-PDM.20, MATSIRO, } \\
\text { mHM, MPI-HM, ORCHIDEE, PCR-GLOBWB, WaterGAP2, WAYS }\end{array}$ \\
\hline Surface runoff & qs $\left(\mathrm{kg} \mathrm{m}^{-2} \mathrm{~s}^{-1}\right) /$ monthly & $\begin{array}{l}\text { CLM4.5, CLM5.0, CWatM, DBH, H08, JULES-W1, LPJmL, Mac-PDM.20, MATSIRO, } \\
\text { MPI-HM, ORCHIDEE, PCR-GLOBWB, WaterGAP2. WAYS }\end{array}$ \\
\hline Subsurface runoff & qsb $\left(\mathrm{kg} \mathrm{m}^{-2} \mathrm{~s}^{-1}\right) /$ monthly & $\begin{array}{l}\text { CLM4.5, CLM5.0, CWatM, DBH, H08, JULES-W1, LPJmL, Mac-PDM.20, MATSIRO, } \\
\text { mHM, MPI-HM, ORCHIDEE, ORCHIDEE-DGVM, PCR-GLOBWB, WaterGAP2, } \\
\text { WAYS }\end{array}$ \\
\hline Groundwater recharge & $\mathrm{qr}\left(\mathrm{kg} \mathrm{m}^{-2} \mathrm{~s}^{-1}\right) /$ monthly & $\begin{array}{l}\text { CLM4.5, CLM5.0, CWatM, DBH, H08, JULES-W1, LPJmL, MATSIRO, mHM, MPI- } \\
\text { HM, ORCHIDEE, PCR-GLOBWB, WaterGAP2, WAYS }\end{array}$ \\
\hline Groundwater runoff & $\mathrm{qg}\left(\mathrm{kg} \mathrm{m}^{-2} \mathrm{~s}^{-1}\right) /$ monthly & $\begin{array}{l}\text { CLM4.5, CLM5.0, CWatM, DBH, H08, JULES-W1, LPJmL, MATSIRO, mHM, MPI- } \\
\text { HM, ORCHIDEE, PCR-GLOBWB, WaterGAP2, WAYS }\end{array}$ \\
\hline Discharge (gridded) & $\operatorname{dis}\left(\mathrm{m}^{3} \mathrm{~s}^{1}\right)$ & $\begin{array}{l}\text { CLM4.5, CLM5.0, CWatM, DBH, H08, LPJmL, MATSIRO, mHM, MPI-HM, } \\
\text { ORCHIDEE, PCR-GLOBWB, WaterGAP2 }\end{array}$ \\
\hline $\begin{array}{l}\text { Monthly maximum of daily } \\
\text { discharge }\end{array}$ & maxdis $\left(\mathrm{m}^{3} \mathrm{~s}^{1}\right) /$ monthly & $\begin{array}{l}\text { CLM4.5, CLM5.0, CWatM, DBH, H08, JULES-W1, LPJmL, MATSIRO, mHM, MPI- } \\
\text { HM, ORCHIDEE, PCR-GLOBWB, WaterGAP2 }\end{array}$ \\
\hline $\begin{array}{l}\text { Monthly minimum of daily } \\
\text { discharge }\end{array}$ & mindis $\left(\mathrm{m}^{3} \mathrm{~s}^{-1}\right) /$ monthly & $\begin{array}{l}\text { CLM4.5, CLM5.0, CWatM, DBH, H08, JULES-W1, LPJmL, MATSIRO, mHM, MPI- } \\
\text { HM, ORCHIDEE, PCR-GLOBWB, WaterGAP2 }\end{array}$ \\
\hline Evapotranspiration & evap $\left(\mathrm{kg} \mathrm{m}^{-2} \mathrm{~s}^{-1}\right) /$ monthly & $\begin{array}{l}\text { CLM4.5, CLM5.0, CWatM, DBH, H08, JULES-W1, LPJmL, Mac-PDM.20, MATSIRO, } \\
\text { mHM, MPI-HM, ORCHIDEE, PCR-GLOBWB, WaterGAP2, WAYS }\end{array}$ \\
\hline Potential Evapotranspiration & potevap $\left(\mathrm{kg} \mathrm{m}^{-2} \mathrm{~s}^{-1}\right) /$ monthly & $\begin{array}{l}\text { CLM4.5, CLM5.0, CWatM, DBH, H08, JULES-W1, LPJmL, Mac-PDM.20, MATSIRO, } \\
\text { mHM, MPI-HM, ORCHIDEE, PCR-GLOBWB, WaterGAP2, WAYS }\end{array}$ \\
\hline Soil moisture & soilmoist $\left(\mathrm{kg} \mathrm{m}^{-2}\right) /$ monthly & $\begin{array}{l}\text { CLM4.5, CLM5.0, CWatM, DBH, H08, JULES-W1, LPJmL, Mac-PDM.20, MATSIRO, } \\
\text { mHM, MPI-HM, ORCHIDEE, PCR-GLOBWB, WaterGAP2 }\end{array}$ \\
\hline Soil moisture, root zone & rootmoist $\left(\mathrm{kg} \mathrm{m}^{-2}\right) /$ monthly & $\begin{array}{l}\text { CLM4.5, CLM5.0, CWatM, DBH, H08, JULES-W1, LPJmL, Mac-PDM.20, MATSIRO, } \\
\text { mHM, MPI-HM, ORCHIDEE, PCR-GLOBWB, WaterGAP2, WAYS }\end{array}$ \\
\hline $\begin{array}{l}\text { Frozen soil moisture for each } \\
\text { layer }\end{array}$ & soilmoistfroz $\left(\mathrm{kg} \mathrm{m}^{-2}\right)$ / monthly & $\begin{array}{l}\text { CLM4.5, CLM5.0, CWatM, DBH, H08, JULES-W1, LPJmL, MATSIRO, ORCHIDEE, } \\
\text { PCR-GLOBWB, WaterGAP2 }\end{array}$ \\
\hline Temperature of Soil & tsl (K) & $\begin{array}{l}\text { CLM4.5, CLM5.0, CWatM, DBH, H08, JULES-W1, LPJmL, MATSIRO, ORCHIDEE, } \\
\text { PCR-GLOBWB, WaterGAP2 }\end{array}$ \\
\hline Snow depth & snd (m) / monthly & $\begin{array}{l}\text { CLM4.5, CLM5.0, CWatM, DBH, H08, JULES-W1, LPJmL, MATSIRO, mHM, MPI- } \\
\text { HM, ORCHIDEE, PCR-GLOBWB, WaterGAP2 }\end{array}$ \\
\hline Snow water equivalent & swe $\left(\mathrm{kg} \mathrm{m}^{-2}\right) /$ monthly & $\begin{array}{l}\text { CLM4.5, CLM5.0, CWatM, DBH, H08, JULES-W1, LPJmL, MATSIRO, mHM, MPI- } \\
\text { HM, ORCHIDEE, PCR-GLOBWB, WaterGAP2 }\end{array}$ \\
\hline Total water storage & tws $\left(\mathrm{kg} \mathrm{m}^{-2}\right) /$ monthly & $\begin{array}{l}\text { CLM4.5, CLM5.0, CWatM, DBH, JULES-W1, LPJmL, MATSIRO, MPI-HM, } \\
\text { ORCHIDEE, PCR-GLOBWB, WaterGAP2 }\end{array}$ \\
\hline Canopy water storage & canopystor / monthly & $\begin{array}{l}\text { CLM4.5, CLM5.0, CWatM, DBH, JULES-W1, LPJmL, MATSIRO, ORCHIDEE, PCR- } \\
\text { GLOBWB, WaterGAP2, WAYS }\end{array}$ \\
\hline Glacier storage & glacierstor / monthly & CLM4.5, CLM5.0, CWatM \\
\hline Groundwater storage & groundwstor / monthly & $\begin{array}{l}\text { CLM4.5, CLM5.0, CWatM, DBH, H08, JULES-W1, LPJmL, MATSIRO, mHM, } \\
\text { ORCHIDEE, PCR-GLOBWB, WaterGAP2 }\end{array}$ \\
\hline Lake storage & lakestor / monthly & CLM4.5, CLM5.0, CWatM, MATSIRO, WaterGAP2 \\
\hline Wetland storage & wetlandstor / monthly & CLM4.5, CLM5.0, CWatM, MPI-HM, ORCHIDEE, PCR-GLOBWB, WaterGAP2 \\
\hline Reservoir storage & reservoirstor / monthly & CWatM, DBH, H08, LPJmL, MATSIRO, PCR-GLOBWB, WaterGAP2 \\
\hline $\begin{array}{l}\text { Annual maximum daily thaw } \\
\text { depth }\end{array}$ & thawdepth (m) / monthly & CLM4.5, CLM5.0, CWatM, DBH, LPJmL, MATSIRO, ORCHIDEE, \\
\hline River storage & riverstor / monthly & $\begin{array}{l}\text { CLM4.5, CLM5.0, CWatM, DBH, H08, LPJmL, MATSIRO, MPI-HM, ORCHIDEE, } \\
\text { PCR-GLOBWB, WaterGAP2 }\end{array}$ \\
\hline Rainfall & $\operatorname{rainf}\left(\mathrm{kg} \mathrm{m}^{-2} \mathrm{~s}^{-1}\right) /$ monthly & $\begin{array}{l}\text { CLM4.5, CLM5.0, CWatM, DBH, H08, JULES-W1, LPJmL, MATSIRO, mHM, MPI- } \\
\text { HM, ORCHIDEE, PCR-GLOBWB, WaterGAP2, WAYS }\end{array}$ \\
\hline Snowfall & snowf $\left(\mathrm{kg} \mathrm{m}^{-2} \mathrm{~s}^{-1}\right) /$ monthly & $\begin{array}{l}\text { CLM4.5, CLM5.0, CWatM, DBH, H08, JULES-W1, LPJmL, Mac-PDM.20, MATSIRO, } \\
\text { mHM, MPI-HM, ORCHIDEE, PCR-GLOBWB, WaterGAP2, WAYS }\end{array}$ \\
\hline Leaf Area Index & lai (-) / monthly & CLM45, ORCHIDEE, WAYS \\
\hline
\end{tabular}


Table S92. Water management variables of the ISMIP2b global water models ${ }^{1}$ with a spatial resolution of $0.5^{\circ} \times 0.5^{\circ}$ and a monthly temporal resolution

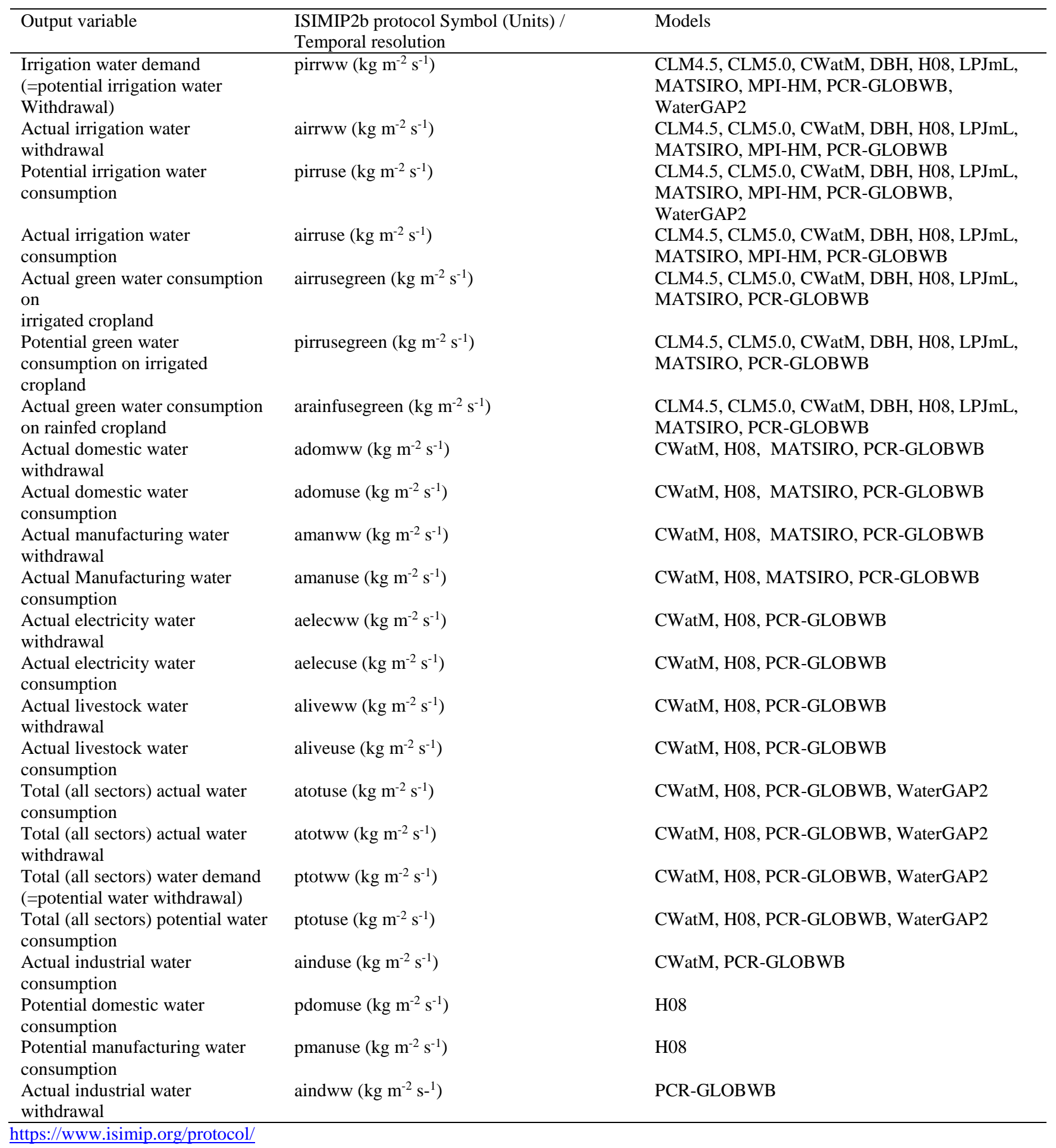


Table S93. Representation of the canopy and snow water storage compartments in the ISIMIP2b GWMs included in the global water sector

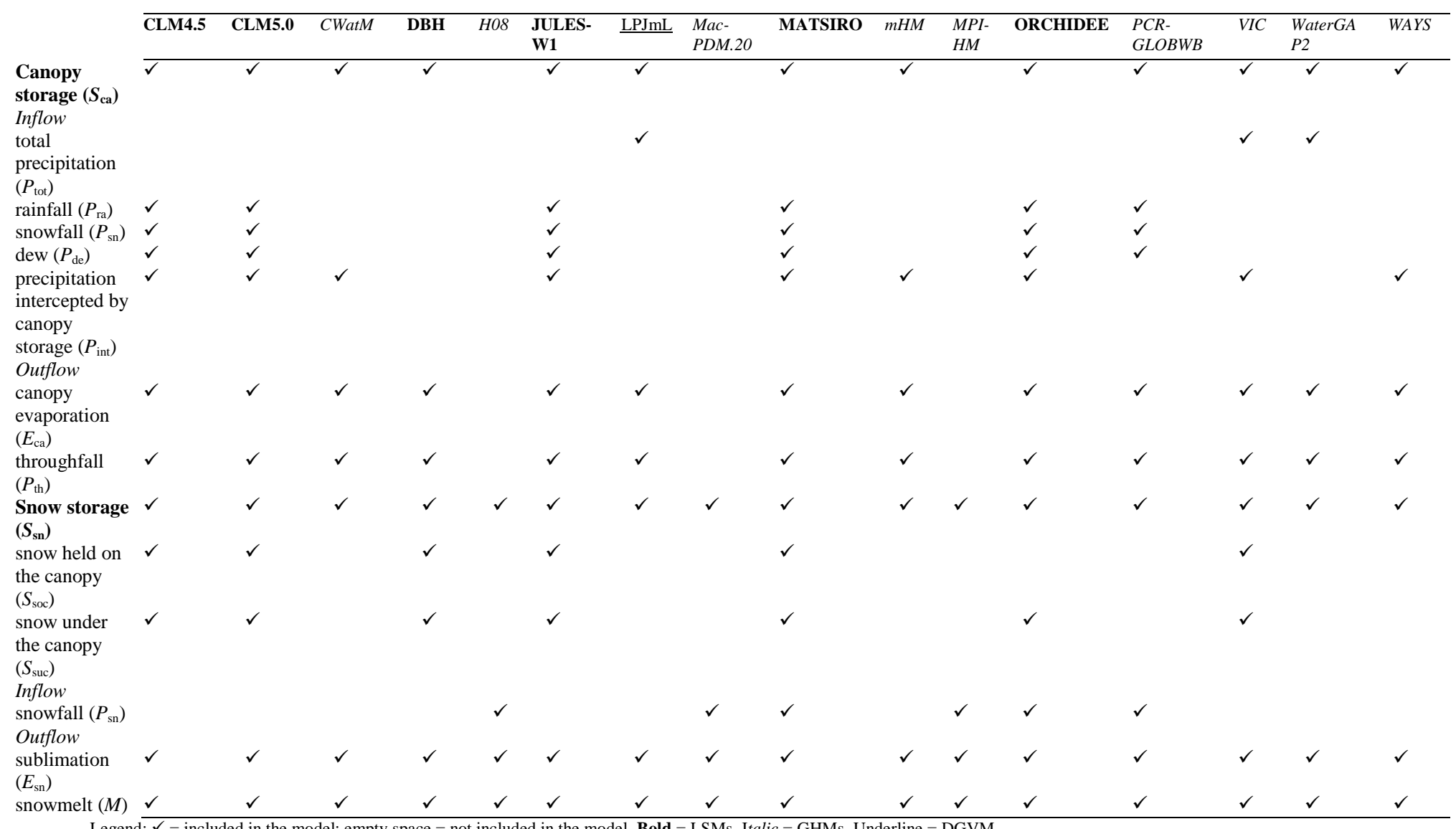

Legend: $\checkmark=$ included in the model; empty space $=$ not included in the model, Bold = LSMs, Italic = GHMs, Underline $=$ DGVM. 
Table S94. Representation of the soil storage in the ISIMIP2b GWMs included in the global water sector

\begin{tabular}{|c|c|c|c|c|c|c|c|c|c|c|c|c|c|c|c|c|}
\hline & CLM4.5 & CLM5.0 & CWatM & DBH & HOS & $\begin{array}{l}\text { JULES } \\
\text {-W1 }\end{array}$ & $\underline{\mathrm{LPJmL}}$ & $\begin{array}{l}\text { Mac- } \\
\text { PDM.20 }\end{array}$ & MATSIRO & $m H M$ & $\begin{array}{l}M P I- \\
H M\end{array}$ & ORCHIDEE & $\begin{array}{l}P C R- \\
\text { GLOBWB }\end{array}$ & VIC & WaterGAP2 & WAYS \\
\hline Soil storage $\left(S_{\mathrm{so}}\right)$ & $\checkmark$ & $\checkmark$ & $\checkmark$ & $\checkmark$ & $\checkmark$ & $\checkmark$ & $\checkmark$ & $\checkmark$ & $\checkmark$ & $\checkmark$ & $\checkmark$ & $\checkmark$ & $\checkmark$ & $\checkmark$ & $\checkmark$ & $\checkmark$ \\
\hline Inflow & $\checkmark$ & $\checkmark$ & $\checkmark$ & $\checkmark$ & $\checkmark$ & $\checkmark$ & $\checkmark$ & $\checkmark$ & $\checkmark$ & $\checkmark$ & $\checkmark$ & $\checkmark$ & $\checkmark$ & $\checkmark$ & $\checkmark$ & $\checkmark$ \\
\hline infiltration $\left(R_{\text {in }}\right)$ & $\checkmark$ & $\checkmark$ & $\checkmark$ & $\checkmark$ & $\checkmark$ & $\checkmark$ & $\checkmark$ & $\checkmark$ & $\checkmark$ & $\checkmark$ & $\checkmark$ & $\checkmark$ & $\checkmark$ & $\checkmark$ & $\checkmark$ & $\checkmark$ \\
\hline $\begin{array}{l}\text { return flow from } \\
\text { human water use } \\
\left(A_{\mathrm{rf}}\right)\end{array}$ & & $\checkmark$ & & & $\checkmark$ & & & & & & & & & & $\checkmark$ & \\
\hline $\begin{array}{l}\text { Outflow } \\
\text { actual } \\
\text { evapotranspiration } \\
\text { (AET) }\end{array}$ & $\checkmark$ & $\checkmark$ & $\checkmark$ & $\checkmark$ & $\checkmark$ & $\checkmark$ & $\checkmark$ & $\checkmark$ & $\checkmark$ & $\checkmark$ & $\checkmark$ & $\checkmark$ & $\checkmark$ & $\checkmark$ & $\checkmark$ & $\checkmark$ \\
\hline $\begin{array}{l}\text { potential } \\
\text { evapotranspiration } \\
\text { (PET) }\end{array}$ & & & $\checkmark$ & $\checkmark$ & $\checkmark$ & $\checkmark$ & $\checkmark$ & $\checkmark$ & $\checkmark$ & $\checkmark$ & $\checkmark$ & $\checkmark$ & $\checkmark$ & $\checkmark$ & $\checkmark$ & $\checkmark$ \\
\hline transpiration $(T)$ & $\checkmark$ & $\checkmark$ & $\checkmark$ & $\checkmark$ & & $\checkmark$ & $\checkmark$ & & $\checkmark$ & & $\checkmark$ & $\checkmark$ & $\checkmark$ & $\checkmark$ & & \\
\hline $\begin{array}{l}\text { evaporation from } \\
\text { soil }\left(E_{\mathrm{so}}\right)\end{array}$ & $\checkmark$ & $\checkmark$ & $\checkmark$ & $\checkmark$ & $\checkmark$ & $\checkmark$ & $\checkmark$ & $\checkmark$ & $\checkmark$ & $\checkmark$ & $\checkmark$ & $\checkmark$ & $\checkmark$ & $\checkmark$ & $\checkmark$ & $\checkmark$ \\
\hline $\begin{array}{l}\text { surface runoff } \\
\left(R_{\mathrm{su}}\right)\end{array}$ & $\checkmark$ & $\checkmark$ & $\checkmark$ & $\checkmark$ & $\checkmark$ & $\checkmark$ & $\checkmark$ & $\checkmark$ & $\checkmark$ & $\checkmark$ & $\checkmark$ & $\checkmark$ & $\checkmark$ & $\checkmark$ & $\checkmark$ & $\checkmark$ \\
\hline $\begin{array}{l}\text { Saturation excess } \\
\text { overland flow } \\
\left(R_{\text {sat }}\right)\end{array}$ & & & & $\checkmark$ & & & $\checkmark$ & & $\checkmark$ & & & & & $\checkmark$ & $\checkmark$ & \\
\hline interflow $\left(R_{\mathrm{if}}\right)$ & & & $\checkmark$ & & & $\checkmark$ & $\checkmark$ & & & $\checkmark$ & & & $\checkmark$ & & & $\checkmark$ \\
\hline $\begin{array}{l}\text { percolation }\left(R_{\mathrm{pe}}\right) \\
\text { groundwater } \\
\text { recharge }\left(R_{\mathrm{gwr}}\right)\end{array}$ & $\checkmark$ & $\checkmark$ & $\begin{array}{l}\checkmark \\
\checkmark\end{array}$ & $\checkmark$ & $\checkmark$ & $\checkmark$ & $\checkmark$ & & $\checkmark$ & & $\begin{array}{l}\checkmark \\
\checkmark\end{array}$ & $\checkmark$ & $\checkmark$ & & $\checkmark$ & $\begin{array}{l}\checkmark \\
\checkmark\end{array}$ \\
\hline
\end{tabular}

Legend: $\checkmark=$ included in the model; empty space $=$ not included in the model, Bold $=$ LSMs, Italic $=$ GHMs, Underline $=$ DGVM. 
Table S95. Representation of the groundwater storage in the ISIMIP2b GWMs included in the global water sector

\begin{tabular}{|c|c|c|c|c|c|c|c|c|c|c|c|c|c|c|c|c|}
\hline & $\overline{\text { CLM4.5 }}$ & CLM5.0 & CWatM & DBH & HOS & $\begin{array}{l}\text { JULES- } \\
\text { W1 }\end{array}$ & $\underline{\text { LPJmL }}$ & $\begin{array}{l}\text { Mac- } \\
\text { PDM.20 }\end{array}$ & MATSIRO & $m H M$ & $\begin{array}{l}M P I- \\
H M\end{array}$ & ORCHIDEE & $\begin{array}{l}P C R- \\
\text { GLOBWB }\end{array}$ & VIC & WaterGAP2 & WAYS \\
\hline $\begin{array}{l}\text { Groundwater } \\
\text { storage }\left(S_{\mathrm{gw}}\right)\end{array}$ & $\checkmark$ & $\checkmark$ & $\checkmark$ & & $\checkmark$ & & & & $\checkmark$ & $\checkmark$ & $\checkmark$ & & $\checkmark$ & $\checkmark$ & $\checkmark$ & $\checkmark$ \\
\hline Inflow & & & & & & & & & $\checkmark$ & $\checkmark$ & & & $\checkmark$ & $\checkmark$ & $\checkmark$ & $\checkmark$ \\
\hline $\begin{array}{l}\text { groundwater } \\
\text { recharge } \\
\text { (seepage) }\left(R_{\mathrm{gwr}}\right)\end{array}$ & $\checkmark$ & $\checkmark$ & $\checkmark$ & $\checkmark$ & $\checkmark$ & $\checkmark$ & $\checkmark$ & $\checkmark$ & $\checkmark$ & $\checkmark$ & $\checkmark$ & $\checkmark$ & $\checkmark$ & $\checkmark$ & $\checkmark$ & $\checkmark$ \\
\hline $\begin{array}{l}\text { return flow } \\
\text { from human } \\
\text { water use }\left(A_{\mathrm{rf}}\right) \\
\text { Outflow }\end{array}$ & & & & & $\checkmark$ & & & & $\checkmark$ & $\checkmark$ & & & $\checkmark$ & $\checkmark$ & $\checkmark$ & $\checkmark$ \\
\hline $\begin{array}{l}\text { capillary rise } \\
\left(R_{\mathrm{cr}}\right)\end{array}$ & $\checkmark$ & $\checkmark$ & $\checkmark$ & & & & & & $\checkmark$ & & & & $\mathrm{x}$ & & & \\
\hline $\begin{array}{l}\text { groundwater } \\
\text { runoff }\left(R_{\mathrm{gw}}\right)\end{array}$ & $\checkmark$ & $\checkmark$ & $\checkmark$ & $\checkmark$ & $\checkmark$ & $\checkmark$ & $\checkmark$ & $\checkmark$ & $\checkmark$ & $\checkmark$ & $\checkmark$ & $\checkmark$ & $\checkmark$ & $\checkmark$ & $\checkmark$ & $\checkmark$ \\
\hline $\begin{array}{l}\text { groundwater } \\
\text { withdrawal for } \\
\text { human water } \\
\text { use }\left(A_{\mathrm{gw}}\right)\end{array}$ & & & & & $\checkmark$ & & & & $\checkmark$ & & & & $\checkmark$ & & $\checkmark$ & \\
\hline
\end{tabular}


Table S96. Representation of the river storage in the ISIMIP2b GWMs included in the global water sector

River

\begin{tabular}{|c|c|c|c|c|c|c|c|c|c|c|c|c|c|c|c|}
\hline CLM4.5 & CLM5.0 & CWatM & DBH & HOS & $\begin{array}{l}\text { JULES- } \\
\text { W1 }\end{array}$ & $\underline{\mathrm{LPJmL}}$ & $\begin{array}{l}M a c- \\
P D M .20\end{array}$ & MATSIRO & $m H M$ & $\begin{array}{l}\text { MPI- } \\
H M\end{array}$ & ORCHIDEE & $\begin{array}{l}P C R- \\
\text { GLOBWB }\end{array}$ & VIC & WaterGAP2 & WAYS \\
\hline$\checkmark$ & $\checkmark$ & $\checkmark$ & $\checkmark$ & $\checkmark$ & & $\checkmark$ & & $v$ & $\checkmark$ & $v$ & $\checkmark$ & 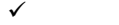 & & $v$ & $\checkmark$ \\
\hline
\end{tabular}

$\left(S_{\text {ri }}\right)$

Inflow

total

precipitation

$\left(P_{\text {tot }}\right)$

rainfall $\left(P_{\text {ra }}\right)$

snowfall

$\left(P_{\mathrm{sn}}\right)$

inflow from

upstream

surface

water bodies

( $\left.Q_{\text {iu }}\right)$

surface

runoff or

overland

flow or fast

runoff $\left(R_{\mathrm{su}}\right)$

Hortonian

overland

flow $\left(R_{\mathrm{ho}}\right)$

Saturation

excess

overland

flow $\left(R_{\text {sat }}\right)$

interflow

( $\left.R_{\text {if }}\right)$

groundwater

runoff $\left(R_{\mathrm{gw}}\right)$

return flow

from human

water use

( $\left.A_{\mathrm{rf}}\right)$

Outflow

streamflow

or outflow

or discharge

$\left(Q_{\text {ri }}\right)$

water

withdrawal

for human

water use

from river

(Ari)

evaporation

from river

$\left(E_{\mathrm{ri}}\right)$ 
Table S97. Representation of the lake storage in the ISIMIP2b GWMs included in the global water sector

Lake

\begin{tabular}{|c|c|c|c|c|c|c|c|c|c|c|c|c|c|c|c|}
\hline CLM4.5 & CLM5.0 & CWatM & DBH & HOS & $\begin{array}{l}\text { JULES- } \\
\text { W1 }\end{array}$ & $\underline{\mathrm{LPJ} m L}$ & $\begin{array}{l}\text { Mac- } \\
\text { PDM.20 }\end{array}$ & MATSIRO & $m H M$ & $\begin{array}{l}M P I- \\
H M \\
\end{array}$ & ORCHIDEE & $\begin{array}{l}P C R- \\
G L O B W B\end{array}$ & VIC & WaterGAP2 & WAYS \\
\hline
\end{tabular}

storage

$\left(S_{\mathrm{la}}\right)$

Inflow

total

precipitation

$\left(P_{\text {tot }}\right)$

rainfall $\left(P_{\mathrm{ra}}\right)$

snowfall

$\left(P_{\mathrm{sn}}\right)$

inflow from

upstream

surface

water

bodies $\left(Q_{\mathrm{iu}}\right)$

surface

runoff or

overland

flow or fast

runoff $\left(R_{\mathrm{su}}\right)$

Hortonian

overland

flow $\left(R_{\mathrm{ho}}\right)$

Saturation

excess

overland

flow $\left(R_{\text {sat }}\right)$

interflow

( $\left.R_{\text {if }}\right)$

groundwater

runoff $\left(R_{\mathrm{gw}}\right)$

return flow

from human

water use

(A $\left.A_{\mathrm{rf}}\right)$

Outflow

evaporation

from lake

$\left(E_{\mathrm{la}}\right)$

outflow

from lake

$\left(Q_{\mathrm{la}}\right)$

groundwater

recharge

( $\left.R_{\mathrm{gwr}}\right)$

water

withdrawal

for human

water use

from lake

$\left(A_{\text {la }}\right)$ 
Table S98. Representation of the reservoir storage in the ISIMIP2b GWMs included in the global water sector

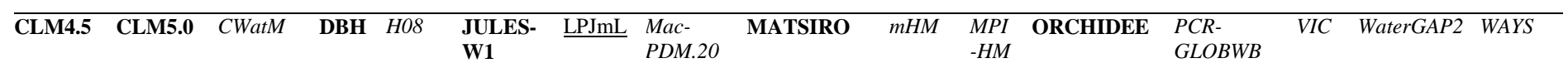

Reservoir

storage $\left(S_{\text {re }}\right)$

Inflow

total

precipitation

$\left(P_{\text {tot }}\right)$

rainfall $\left(P_{\mathrm{ra}}\right)$

snowfall $\left(P_{\mathrm{sn}}\right)$

inflow from

upstream

surface water

bodies $\left(Q_{\mathrm{iu}}\right)$

surface

runoff or

overland

flow or fast

runoff $\left(R_{\mathrm{su}}\right)$

Hortonian

overland

flow $\left(R_{\mathrm{ho}}\right)$

Saturation

excess

overland

flow $\left(R_{\text {sat }}\right)$

interflow

$\left(R_{\text {iu }}\right)$

groundwater

runoff $\left(R_{\mathrm{gw}}\right)$

return flow

from human

water use

$\left(A_{\text {rf }}\right)$

Outflow

evaporation

from

reservoir

$\left(E_{\mathrm{re}}\right)$

outflow from

reservoir

$\left(Q_{\mathrm{re}}\right)$

groundwater

recharge

$\left(R_{\mathrm{gwr}}\right)$

water

withdrawal

for human

water use

from

reservoir

$\left(A_{\text {re }}\right)$

Legend: $\checkmark=$ included in the model; empty space = not included in the model, Bold = LSMs, Italic $=$ GHMs, Underline = DGVMs. 
Table S99. Representation of the wetland storage in the ISIMIP2b GWMs included in the global water sector

\begin{tabular}{|c|c|c|c|c|c|c|c|c|c|c|c|c|c|c|c|}
\hline CLM4.5 & CLM5.0 & CWatM & DBH & $\mathrm{HOS}$ & $\begin{array}{l}\text { JULES } \\
-W 1\end{array}$ & $\underline{\mathrm{LPJmL}}$ & $\begin{array}{l}\text { Mac- } \\
\text { PDM.20 }\end{array}$ & MATSIRO & $m H M$ & $\begin{array}{l}M P I- \\
H M\end{array}$ & ORCHIDEE & $\begin{array}{l}P C R- \\
G L O B W B\end{array}$ & VIC & WaterGAP2 & WAYS \\
\hline
\end{tabular}

\section{Wetland}

storage $\left(S_{\text {we }}\right)$

Inflow

total

precipitation

$\left(P_{\text {tot }}\right)$

rainfall $\left(P_{\text {ra }}\right)$

snowfall $\left(P_{\mathrm{sn}}\right)$

inflow from

upstream

surface water

bodies $\left(Q_{\text {iu }}\right)$

surface runoff

or overland

flow or fast

runoff $\left(R_{\mathrm{su}}\right)$

Hortonian

overland flow

( $\left.R_{\mathrm{ho}}\right)$

Saturation

excess

overland flow

$\left(R_{\text {sat }}\right)$

interflow $\left(R_{\mathrm{if}}\right)$

groundwater

runoff $\left(R_{\mathrm{gw}}\right)$

Outflow

groundwater

recharge $\left(R_{\text {gwr }}\right)$

evapotranspira

tion (PET)

transpiration

(T)

evaporation

from wetland

$\left(E_{\mathrm{we}}\right)$

outflow from

wetland $\left(Q_{\mathrm{we}}\right)$

Legend: $\checkmark=$ included in the model; empty space $=$ not included in the model, Bold $=$ LSMs, I $t a l i c=$ GHMs, Underline $=$ DGVMs. 
Table S100. Human water use sectors included in the Global Water Models - Part I

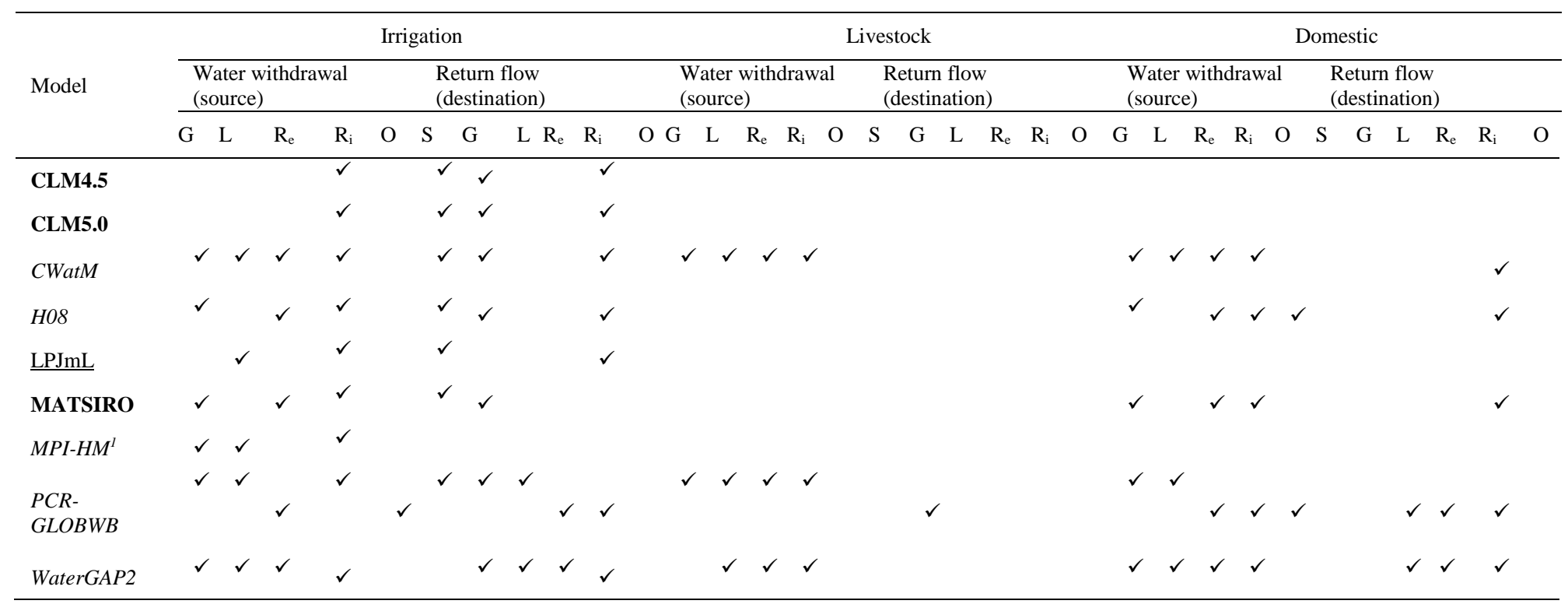

Legend: $\mathrm{G}$ = groundwater; $\mathrm{L}=$ lake; $\mathrm{O}=$ ocean; $\mathrm{R}_{\mathrm{e}}=$ reservoir; $\mathrm{R}_{\mathrm{i}}=$ river; $\mathrm{S}=$ soil; Bold = LSMs; Italic = GHMs; Underline = DGVMs.

Note: 1: MPI-HM extracts water from the wetland storage, which includes the water stored in lakes. 
Table S101. Human water use sectors included in the Global Water Models - Part II

\begin{tabular}{|c|c|c|c|c|c|c|c|c|c|c|c|c|c|c|c|c|c|c|c|c|c|c|c|c|c|c|c|}
\hline \multirow{3}{*}{ Model } & \multicolumn{9}{|c|}{ Manufacturing } & \multicolumn{9}{|c|}{ Electricity } & \multicolumn{9}{|c|}{ Desalination } \\
\hline & \multicolumn{5}{|c|}{$\begin{array}{l}\text { Water withdrawal } \\
\text { (source) }\end{array}$} & \multicolumn{4}{|c|}{$\begin{array}{l}\text { Return flow } \\
\text { (destination) }\end{array}$} & \multicolumn{4}{|c|}{$\begin{array}{c}\text { Water withdrawal } \\
\text { (source) }\end{array}$} & \multicolumn{4}{|c|}{$\begin{array}{l}\text { Return flow } \\
\text { (destination) }\end{array}$} & \multicolumn{3}{|c|}{$\begin{array}{c}\text { Water } \\
\text { withdrawal } \\
\text { (source) }\end{array}$} & \multicolumn{7}{|c|}{$\begin{array}{l}\text { Return flow } \\
\text { (destination) }\end{array}$} \\
\hline & G & $\mathrm{L}$ & $\mathrm{R}_{\mathrm{e}}$ & $\mathrm{R}_{\mathrm{i}}$ & $\mathrm{O}$ & $S \mathrm{G}$ & $\mathrm{L}$ & $\mathrm{R}_{\mathrm{e}}$ & $\mathrm{R}_{\mathrm{i}} \mathrm{O}$ & G & $\mathrm{L}$ & $\mathrm{R}_{\mathrm{e}}$ & $\mathrm{R}_{\mathrm{i}} \mathrm{O}$ & $\mathrm{O} S$ & G & $L \quad R_{e}$ & $\mathrm{R}_{\mathrm{i}} \mathrm{O}$ & G & $L R_{e} R_{i}$ & $\mathrm{O}$ & ISW & $\mathrm{S}$ & G & $\begin{array}{ll}L & R_{e}\end{array}$ & $\mathrm{R}_{\mathrm{i}}$ & $\mathrm{O}$ & ISW \\
\hline CWatM & $\checkmark$ & $\checkmark$ & $\checkmark$ & $\checkmark$ & & & & & $\checkmark$ & $\checkmark$ & $\checkmark$ & $\checkmark$ & $\checkmark$ & & & & $\checkmark$ & & & & & & & & & & \\
\hline HOS & $\checkmark$ & & $\checkmark$ & $\checkmark$ & & & & & $\checkmark$ & & & & & & & & & & & $\checkmark$ & & & & & & $\checkmark$ & \\
\hline MATSIRO & $\checkmark$ & & $\checkmark$ & $\checkmark$ & & & & & $\checkmark$ & & & & & & & & & & & & & & & & & & \\
\hline$P C R-G L O B W B$ & $\checkmark$ & $\checkmark$ & $\checkmark$ & $\checkmark$ & $\checkmark$ & & $\checkmark$ & $\checkmark$ & $\checkmark$ & $\checkmark$ & $\checkmark$ & $\checkmark$ & $\checkmark \checkmark$ & $\checkmark$ & & $\checkmark \checkmark$ & $\checkmark$ & & & $\checkmark$ & & & & & & $\checkmark$ & \\
\hline WaterGAP2 & $\checkmark$ & $\checkmark$ & $\checkmark$ & $\checkmark$ & & & $\checkmark$ & $\checkmark$ & $\checkmark$ & & $\checkmark$ & $\checkmark$ & $\checkmark$ & & & $\checkmark \checkmark \checkmark$ & $\checkmark$ & & & & & & & & & & \\
\hline
\end{tabular}

Legend: $\mathrm{G}=$ groundwater; $\mathrm{ISW}=$ inland saline water; $\mathrm{L}=$ lake; $\mathrm{O}=$ ocean; $\mathrm{R}_{\mathrm{e}}=$ reservoir; $\mathrm{R}_{\mathrm{i}}=$ river; $\mathrm{S}=$ soil; Bold $=\mathrm{LSMs}$; Italic = GHMs; Underline = DGVMs. 
Table S102. Potential future research in global hydrological modeling - Part I

\begin{tabular}{|c|c|c|c|c|c|c|c|c|c|}
\hline Model & $\begin{array}{l}\text { Input } \\
\text { data }\end{array}$ & $\begin{array}{l}\text { Output } \\
\text { data }\end{array}$ & $\begin{array}{l}\text { Temporal } \\
\text { resolution }\end{array}$ & $\begin{array}{l}\text { Spatial } \\
\text { resolution }\end{array}$ & Calibration & $\begin{array}{l}\text { Canopy } \\
\text { storage }\end{array}$ & Soil storage & $\begin{array}{l}\text { Snow } \\
\text { storage }\end{array}$ & $\begin{array}{l}\text { Glacier } \\
\text { storage }\end{array}$ \\
\hline CLM4.5 & $\checkmark$ & $\checkmark$ & & & $\checkmark$ & $\checkmark$ & $\checkmark$ & & \\
\hline CLM5.0 & $\checkmark$ & $\checkmark$ & & & $\checkmark$ & $\checkmark$ & $\checkmark$ & & \\
\hline CWatM & & $\checkmark$ & & & $\checkmark$ & & & & \\
\hline DBH & $\checkmark$ & $\checkmark$ & & & $\checkmark$ & & & & \\
\hline HOS & $\checkmark$ & & & $\checkmark$ & $\checkmark$ & & & & \\
\hline JULES-W1 & & $\checkmark$ & & & $\checkmark$ & & & & \\
\hline LPJmL & $\checkmark$ & $\checkmark$ & & $\checkmark$ & & & & & \\
\hline$\overline{M a c-P D M .20}$ & & & & & & & & & \\
\hline MATSIRO & $\checkmark$ & $\checkmark$ & & & & & & & \\
\hline $\begin{array}{l}m H M \\
M P I-H M\end{array}$ & & & & $\checkmark$ & & $\checkmark$ & & $\checkmark$ & $\checkmark$ \\
\hline ORCHIDEE & & & & & $\checkmark$ & & & & \\
\hline $\begin{array}{l}P C R-G L O B W B \\
\text { VIC }\end{array}$ & $\checkmark$ & & $\checkmark$ & $\checkmark$ & & & $\checkmark$ & $\checkmark$ & \\
\hline $\begin{array}{l}\text { WaterGAP2 } \\
\text { WAYS }\end{array}$ & $\checkmark$ & $\checkmark$ & & & & & & & \\
\hline
\end{tabular}

Legend: Bold $=$ LSMs, Italic $=$ GHMs, Underline $=$ DGVMs.

Table S103. Potential future research in global hydrological modeling - Part II

\begin{tabular}{|c|c|c|c|c|c|c|c|}
\hline Model & $\begin{array}{l}\text { Groundwater } \\
\text { storage }\end{array}$ & $\begin{array}{l}\text { River } \\
\text { storage }\end{array}$ & $\begin{array}{l}\text { Runoff } \\
\text { scheme }\end{array}$ & $\begin{array}{l}\text { Lakes } \\
\text { storage }\end{array}$ & $\begin{array}{l}\text { Reservoir } \\
\text { storage }\end{array}$ & $\begin{array}{l}\text { Wetland } \\
\text { storage }\end{array}$ & Water use model \\
\hline CLM4.5 & $\checkmark$ & $\checkmark$ & $\checkmark$ & $\checkmark$ & $\checkmark$ & $\checkmark$ & $\checkmark$ \\
\hline CLM5.0 & $\checkmark$ & $\checkmark$ & $\checkmark$ & $\checkmark$ & $\checkmark$ & $\checkmark$ & $\checkmark$ \\
\hline \multicolumn{8}{|l|}{ CWatM } \\
\hline DBH & & & & $\checkmark$ & & $\checkmark$ & $\checkmark$ \\
\hline \multicolumn{8}{|l|}{ HOS } \\
\hline $\begin{array}{l}\text { JULES-W1 } \\
\text { LPJmL }\end{array}$ & & $\checkmark$ & $\checkmark$ & & & & \\
\hline Mac-PDM.20 & & & & & & & $\checkmark$ \\
\hline MATSIRO & $\checkmark$ & & & & & & $\checkmark$ \\
\hline$m H M$ & $\checkmark$ & & & $\checkmark$ & $\checkmark$ & $\checkmark$ & $\checkmark$ \\
\hline MPI-HM & & & & & $\checkmark$ & & \\
\hline \multicolumn{8}{|l|}{ ORCHIDEE } \\
\hline$P C R-G L O B W B$ & $\checkmark$ & & $\checkmark$ & & $\checkmark$ & & \\
\hline VIC & $\checkmark$ & & & & $\checkmark$ & & $\checkmark$ \\
\hline WaterGAP2 & $\checkmark$ & & & & & & $\checkmark$ \\
\hline WAYS & & & & & & & $\checkmark$ \\
\hline
\end{tabular}

Legend: Bold $=$ LSMs, Italic $=$ GHMs, Underline $=$ DGVMs. 